\title{
SERVO PATTERN ENHANCEMENTS FOR HIGH AREAL DENSITY HARD DISK DRIVES
}

\section{MA KUN}

School of Electrical \& Electronic Engineering

A thesis submitted to the Nanyang Technological University in partial fulfilment of the requirements for the degree of Doctor of Philosophy 

To my family,

for their encouragement and love. 



\section{Acknowledgements}

First and foremost, I would like to express my deepest appreciation and sincere gratitude to my supervisor, Prof. Youyi Wang, for his invaluable support, guidance, and encouragement during my doctoral study. His profound conceptions and insightful judgement towards academic research are impressive and admirable. I would also like to thank my co-supervisor, Dr. Guoxiao Guo from Western Digital, for his great ideas, guidance, and contribution of time in the past a few years. His knowledge, determination, and enthusiasm towards excellence make this journey a very fruitful one for me.

Moreover, I would like to thank my school, School of Electrical and Electronic Engineering, Nanyang Technological University, my company, Western Digital for providing this precious opportunity and financial assistance in pursuit of my doctoral degree.

I would like to thank all my bosses and colleagues from Western Digital Singapore R\&D center in Science Park, Dr. Qingwei Jia, Ms. Wai Ee Wong, Dr. Jianyi Wang, Dr. Hui Li, Dr. Fan Hong, Mr. Shanjiao Jiang, Mr. Yanfeng Zhang, Mr Junpeng Niu and Mr Bin $\mathrm{Hu}$ for the insightful discussion and cooperation. It has been a great pleasure and wonderful experience to work together with all of you.

Last but not least, I would like to express my heartfelt appreciation to my parents and my wife for their love, support and inspiration. They make me solely focus on the research and work by releasing me from any other burdens. I also want to thank my little cute daughter for giving me so much joy and happiness during this long journey. 



\section{Table of Contents}

Acknowledgements $\quad$ i

Table of Contents iii

Summary viii

List of Figures $\quad$ x

List of Tables $\quad$ xvii

Nomenclature xviii

1 Introduction 1

1.1 Background .......................... 1

1.1.1 Shingled Magnetic Recording . . . . . . . . . . . . . 3

1.1.2 Two-Dimensional Magnetic Recording . . . . . . . . . . . . 4

1.1.3 Heat-Assisted Magnetic Recording . . . . . . . . . . 5

1.1.4 Bit Patterned Media . . . . . . . . . . . . . . . 6 
1.1.5 HDD Technology Roadmap . . . . . . . . . . . . . 7

1.2 Motivation . . . . . . . . . . . . . . . . . . 8

1.3 Objectives . . . . . . . . . . . . . . . . . . . 10

1.4 Contributions . . . . . . . . . . . . . . . . . 11

1.5 Organization of the Thesis . . . . . . . . . . . . . . 13

2 HDD Servo Control System and HAMR Technology 15

2.1 HDD Servo Control System _ . . . . . . . . . . . . . . 15

2.1 .1 Introduction . . . . . . . . . . . . . . . . 15

2.1.2 Servo Sector and Position Error Signal (PES) . . . . . . . . 16

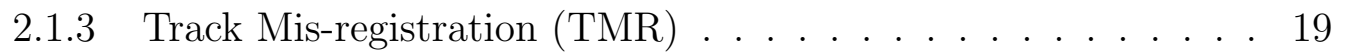

2.1.4 Wedge Repeatable Run-out (WRRO) Compensation . . . . . 19

2.2 HAMR Technology . . . . . . . . . . . . . . . . . . 22

2.2.1 Principle of HAMR . . . . . . . . . . . . . . . . . . . . 23

2.2 .2 Media for HAMR . . . . . . . . . . . . . . . 26

2.2.3 Light Delivery System for HAMR . . . . . . . . . . . . . . . 27

2.2 .4 NFT Design . . . . . . . . . . . . . . . . . . . . . . 29

2.3 Experiment Setup . . . . . . . . . . . . . . . . 31

2.4 Summary . . . . . . . . . . . . . . . . . . . . . . . . 32

3 HAMR Writing Modeling and Identification 33

3.1 Introduction . . . . . . . . . . . . . . . . . . 33

Nanyang Technological University Singapore 
3.2 HAMR Writing Model Identification . . . . . . . . . . . . . . . 37

3.2.1 Structure of HAMR Writing Model . . . . . . . . . . . . . . . 39

3.2.1.1 NFT Protrusion . . . . . . . . . . . . . . 39

3.2.1.2 Power-to-Signal Conversion . . . . . . . . . . . 40

3.2.1.3 Heat Transient Process . . . . . . . . . . . . . . 45

3.2.1.4 Energy Transfer Efficiency . . . . . . . . . . . . 46

3.2.2 Experiment Setup . . . . . . . . . . . . . . . . . 4 47

3.2 .3 System Identification . . . . . . . . . . . . . . . . . 48

3.2.3.1 Identify $f(x)$, NFT Protrusion Gain and Time Constant, and Threshold Thermal Power . . . . . . . . 50

3.2.3.2 Identify Heat Transient Gain and Time Constant . . 52

3.2.4 Identification Results . . . . . . . . . . . . . . 54

3.2.5 Model Verification . . . . . . . . . . . . . . . . . 55

3.3 Slow Protrusion Parameters Identification . . . . . . . . . . . . . . . 59

3.4 Conclusion .......................... 63

4 Applications for HAMR Writing Model 65

4.1 Introduction . . . . . . . . . . . . . . . 65

4.2 Compensation of Protrusion Caused NMS Change . . . . . . . . . . 66

4.2.1 Calculate NFT and Slow Protrusions . . . . . . . . . 66

4.2.2 RRO Field Write, Servo Pattern Write and User Data Field

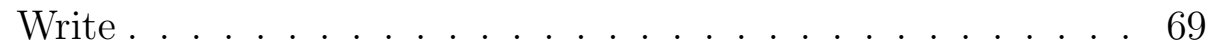


4.2.3 Compensation of NFT Protrusion Caused NMS Change . . . . 71

4.2.4 Compensation of Slow Protrusion Caused NMS Change . . . . 72

4.3 Compensation of Fast Transient Impact . . . . . . . . . . . . . . . . 73

4.3.1 Laser Current Overshoot Design via Pole Placement Method . 74

4.3.2 Laser Current Profile Design via Deconvolution Method . . . . 79

4.4 Conclusion . . . . . . . . . . . . . . . . . . . . . . . . . 84

5 Burst Pattern Non-linearity Improvement $\quad 85$

5.1 Introduction . . . . . . . . . . . . . . . . . . . . . 85

5.2 PES Non-Linearity Characterization . . . . . . . . . . . 87

5.3 Ideal Burst Pattern . . . . . . . . . . . . . . . . . . . . . . . 89

5.4 Improve Written-in Burst Pattern Non-linearity . . . . . . . . . . . . 96

5.5 Conclusion . . . . . . . . . . . . . . . . . . . . 98

6 Continuous Wedge Repeatable Run-out Compensation 99

6.1 Introduction . . . . . . . . . . . . . . . . . . . . . . . 99

6.2 Multi-Track Blending for CWRRO . . . . . . . . . . . . . . . . 102

6.3 Conclusion . . . . . . . . . . . . . . . . . . . . 110

7 Conclusions and Future Works

7.1 Conclusions . . . . . . . . . . . . . . . . . . . . . . . . . 113

7.2 Suggestions for Future Research . . . . . . . . . . . . . . . . 115 
Bibliography

Appendix A - Burst Distortion with 2 to 5X Harmonics 


\section{Summary}

The growth of areal density (AD) in hard disk drive (HDD), which measures the data bits per unit area, has slowed down considerably in recent years from the average of $30 \%$ annual increase rate achieved in past decades. The main reason is that the current perpendicular magnetic recording (PMR) technology has reached its bottleneck of the so-called superparamagnetism. In the meantime, HDD is facing a strong competition and high market share challenge from solid state drive (SSD). As a result, HDD industry is striving to improve the AD with a few technologies such as shingled magnetic recording (SMR), two-dimensional magnetic recording (TDMR), heat-assisted magnetic recording (HAMR) and bit patterned media (BPM). Among all of these, HAMR is the most viable and promising technology to bring the AD beyond a few terabyte/in ${ }^{2}$ level in the coming decade. When

the $\mathrm{AD}$ is $>1$ terabyte $/ \mathrm{in}^{2}$, the track width is expected to be smaller than a few tens of nanometers. Being a mechanical device, ever decreasing track width has imposed more stringent requirements on the servo system for the better track-following performance of read and write operations.

This thesis first focuses on writing good quality servo patterns such as burst field and repeatable run-out (RRO) compensation field under HAMR writing conditions. The HAMR writing mechanism is fundamentally different from the PMR. For PMR, the written-in signal quality is mainly determined by the strength and the gradient of the writing magnetic field. However, for HAMR, although the writing magnetic field is important, the dominating factor for recording quality is determined by the media thermal profile. The media thermal profile is in turn determined by the thermal properties of the media stack and the thermal power delivered to the media. Given the write head design, and especially the near field transducer (NFT), the thermal power delivered to the media can be controlled through input laser power and the spacing between the NFT and the media. Unfortunately, the NFT-to-media 
spacing (NMS) keeps changing due to the laser-induced heat sources, which makes the written-in signal quality control a much more complex task compared with that of PMR. In order to predict and control the written-in signal quality, the dynamic model for the HAMR writing process are constructed and identified. Using the HAMR writing process model, the NMS and input laser current can be optimized to achieve more consistent written-in signal quality. The focus of this thesis is to write high quality RRO field and servo burst field from the first bit to the last bit, from the first servo sector to the last sector or even from the first track to the last track. However, this methodology applies to data writing as well.

Next, the servo track-following performance is enhanced by improving the burst pattern non-linearity and RRO compensation scheme. The position error signal (PES), which is the only feedback signal for the closed-loop servo control to tell the head position in the radial direction, is demodulated from the burst pattern. The analysis will be performed on how to reduce the non-linearity of the written-in burst pattern to minimize the induced PES demodulation error and improve the head position accuracy. In addition, due to the reduction of space between adjacent tracks by SMR technology and the smaller track width in the high AD HAMR drive, the current RRO compensation scheme and technology cannot work properly due to the limited off-track read capability (OTRC). A new RRO compensation scheme, continuous wedge RRO (CWRRO), is proposed and discussed in this thesis. This scheme is a revolutionary change from the current RRO compensation scheme and has the advantage of providing the RRO compensation values for any off-track locations. 


\section{List of Figures}

1.1 HDD areal density growth $[2] \ldots \ldots \ldots \ldots \ldots \ldots$

1.2 Concept of Shingled Magnetic Recording [6] . . . . . . . . . . 3

1.3 Concept of Two-Dimensional Magnetic Recording [7]. . . . . . . . . 4

1.4 Illustration of Two-Dimensional signal processing for TDMR [7]. . . 5

1.5 PMR vs. HAMR $[6] \ldots \ldots \ldots \ldots \ldots \ldots \ldots$

1.6 Concept of Bit Pattern Media [15]. . . . . . . . . . . . . . . . 7

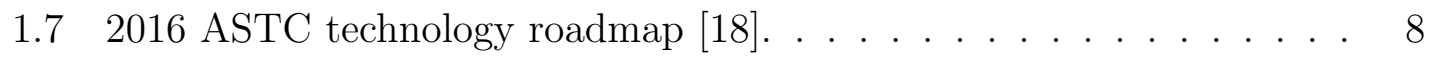

1.8 Block diagram of servo control system. The burst pattern and RRO compensation are the focuses of this thesis to improve the servo per-

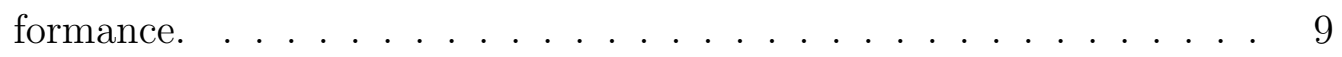

2.1 Internal structure of 2.5 inch 500 gigabyte Western Digital Scorpio Blue hard drive. . . . . . . . . . . . . . . . . . . 16

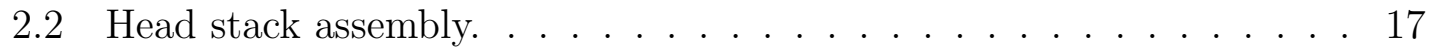

2.3 Embedded servo and servo sector/wedge. . . . . . . . . . . . 18

2.4 Servo written track and repeatable run-out (RRO). . . . . . . . 20 
2.5 Block diagram for RRO compensation using adaptive feedforward

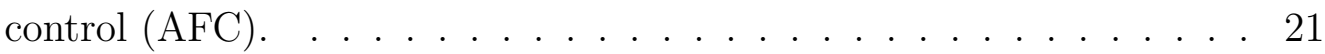

2.6 Block diagram for RRO compensation. . . . . . . . . . . . . . . . . . 21

2.7 Block diagram for RRO compensation value learning. . . . . . . . . . 22

2.8 Principle of HAMR technology [5] . . . . . . . . . . . . . . . . . . . 24

2.9 Thermal and magnetic field down-track profile during writing process

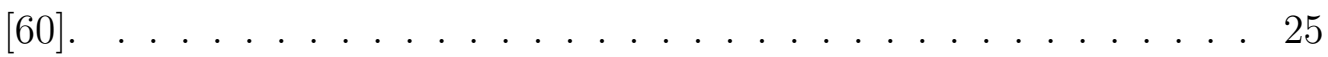

2.10 Structure of HAMR media $[5] \ldots \ldots \ldots$. . . . . . . . . 27

2.11 Light delivery system of HAMR [76]. . . . . . . . . . . . . . . . . . . 28

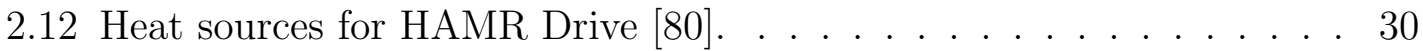

2.13 Block diagram of the experiment setup. . . . . . . . . . . . 31

3.1 Dynamic model for HAMR writing process. . . . . . . . . . . . . . 37

3.2 Input power sequence. . . . . . . . . . . . . . . . . . . . 40

3.3 (a) 1-D thermal profiles with different effective thermal power - $T(y)$, $T_{1}=600 K, T_{2}=700 K$; (b) Corresponding 1-D magnetic pattern $M(y) \ldots \ldots \ldots \ldots \ldots \ldots \ldots \ldots$

3.4 True signal magnitude $S_{T}$ vs. normalized effective thermal power $P_{E}$. $\quad 45$

3.5 Heat transient process illustration. . . . . . . . . . . . . . 46

3.6 Experiment setup. . . . . . . . . . . . . . . . . . . . . . . . . . . . 48

3.7 One example of read-back signal magnitude. . . . . . . . . . . . . . . 50

3.8 Energy transfer efficiency function $f\left(d_{n m s}\right) \ldots \ldots \ldots$. . . . . . . . 55 
3.9 Compare $P_{E}$ between drive data and model output at $t=0.1 \mu s$ and

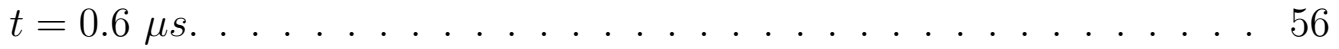

3.10 Compare $P_{E}$ between drive data and model output at $t=1.65 \mu \mathrm{s}$

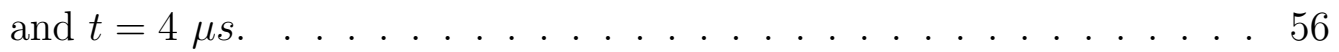

3.11 Model verification of over-shoot time constants of $50 \mathrm{~ns}$ and $600 \mathrm{~ns}$ with default TFC power. . . . . . . . . . . . . . . 57

3.12 Model verification of $10 \%$ and $20 \%$ over-shoot amplitude by increase TFC power by $10 \mathrm{~mW} \ldots \ldots \ldots$. . . . . . . . . . . . 58

3.13 Model verification of $10 \%$ and $20 \%$ over-shoot amplitude by decrease TFC power by $10 \mathrm{~mW} \ldots \ldots \ldots$. . . . . . . . . . . . 59

3.14 Slow protrusion gain measurement. . . . . . . . . . . . . . 60

3.15 Slow protrusion vs. laser power. . . . . . . . . . . . . . 61

3.16 Slow protrusion time constant measurement. . . . . . . . . . . . 62

3.17 Slow protrusion vs. time. . . . . . . . . . . . . . . 63

4.1 Illustration of normalized NFT protrusion and decay. $P$ is the servo sampling time. $T$ is the write gate turned on time for each servo wedge or data sector. . . . . . . . . . . . . . . . . 67

4.2 Normalized fast NFT protrusion amount at the end of writing for each servo wedge or data sector. Comparisons are made among RRO field write, servo pattern write and user data field write. . . . . . . . 70

4.3 Normalized slow protrusion amount at the end of writing for each servo wedge or data sector. Comparisons are made among RRO field write, servo pattern write and user data field write. . . . . . . . . 71 
4.4 Using TFC to compensate for the slow protrusion. (a) Heater power profile. Negative value means the heater power is reduced from initial value; (b) slow protrusion will cause the NMS to decrease whereas the reduction of TFC heater power will cause the NMS to increase. The net NMS change is approaching to $0 \ldots \ldots \ldots . \ldots 73$

4.5 Illustration of exponential decay overshoot components added on top of the step input and their Laplace transform. . . . . . . . . . . 75

4.6 Simplified model with laser power as input and media thermal power as output. . . . . . . . . . . . . . . . 76

4.7 Relationship between input laser current and output laser power. Threshold current $I_{t h}$ and the gain $g_{d}$ are calibrated and known in advance. . . . . . . . . . . . . . . . . 78

4.8 Comparison of different input laser power profiles. The two optimized profiles by pole placement and deconvolution method are able to produce approximately step change in signal magnitude at the model output. No overshoot and single overshoot input profiles are also included for comparison. . . . . . . . . . . . . . . . 81

4.9 Signal magnitude transient response comparisons for different input laser profiles: no overshoot applied, only one overshoot exponential decay component applied and input laser profiles derived by both pole place and deconvolution methods. . . . . . . . . . . . . 82

5.1 Illustration of Null phase burst demodulation [32] . . . . . . . . . . . 86

5.2 Concept of burst flatness. . . . . . . . . . . . . . . . . . 88

5.3 Concept of burst asymmetry. . . . . . . . . . . . . . . . . . . . . 88 
5.4 Demodulation and non-linearity of baseline Null burst pattern. . . . . 90

5.5 PES error vs distortion amplitude. . . . . . . . . . . . . . . . 93

5.6 Burst with $7.5 \% 5 X$ cos harmonic distortion. . . . . . . . . . . . . . 94

5.7 Burst with $-7.5 \% 3 X$ cos harmonic distortion. . . . . . . . . . . 95

5.8 Burst profile comparison for different track width. . . . . . . . . . 96

$5.9 B_{1}$ coefficient of Fourier series for $P E S_{c}$ versus the ratio of reader to track width. . . . . . . . . . . . . . . . . . . 98

6.1 Illustration of read-WRRO and write-WRRO. . . . . . . . . . . . 100

6.2 Illustration of CWRRO. . . . . . . . . . . . . . . . . . . . 102

6.3 Concept of linear interpolation. . . . . . . . . . . . . . . . 104

6.4 Correlation coefficient of WRRO at different distance. . . . . . . . . . 104

6.5 TMR performance comparison between TWRRO and basic CWRRO scheme. . . . . . . . . . . . . . . . . 105

6.6 Concept of MTB. . . . . . . . . . . . . . . . . 106

6.7 Example of MTB coefficients. . . . . . . . . . . . . . . . . 109

6.8 TMR comparison between CWRRO basic scheme and MTB. . . . . . 110

6.9 Cross surface average TMR comparison between CWRRO basic scheme and MTB. . . . . . . . . . . . . . . . . . . . . . . . . . .

6.10 Cross surface residue MSE comparison between CWRRO basic scheme and MTB. . . . . . . . . . . . . . . . . . . . . . . . . 
A1 Demodulation and non-linearity of Null burst pattern with $-10 \%$ 2nd order cos harmonic distortion . . . . . . . . . . . . . . . . 138

A2 Demodulation and non-linearity of Null burst pattern with $10 \%$ 2nd order cos harmonic distortion . . . . . . . . . . . . . . 139

A3 Demodulation and non-linearity of Null burst pattern with $-10 \%$ 2nd order $\sin$ harmonic distortion . . . . . . . . . . . . . . . . 140

A4 Demodulation and non-linearity of Null burst pattern with $10 \%$ 2nd order sin harmonic distortion . . . . . . . . . . . . . . . . . 141

A5 Demodulation and non-linearity of Null burst pattern with $-10 \%$ 3rd order cos harmonic distortion . . . . . . . . . . . . . . . . . . 142

A6 Demodulation and non-linearity of Null burst pattern with 10\% 3rd order cos harmonic distortion . . . . . . . . . . . . . . . 143

A7 Demodulation and non-linearity of Null burst pattern with $-10 \%$ 3rd order $\sin$ harmonic distortion . . . . . . . . . . . . . . . 144

A8 Demodulation and non-linearity of Null burst pattern with $10 \%$ 3rd order $\sin$ harmonic distortion . . . . . . . . . . . . . . 145

A9 Demodulation and non-linearity of Null burst pattern with $-10 \%$ th order cos harmonic distortion . . . . . . . . . . . . . . . 146

A10 Demodulation and non-linearity of Null burst pattern with $10 \%$ 4th order cos harmonic distortion . . . . . . . . . . . . . . . . 147

A11 Demodulation and non-linearity of Null burst pattern with $-10 \%$ th order $\sin$ harmonic distortion . . . . . . . . . . . . . . . . . . 148

A12 Demodulation and non-linearity of Null burst pattern with $10 \%$ th order $\sin$ harmonic distortion . . . . . . . . . . . . . . . . . . . . 149 
A13 Demodulation and non-linearity of Null burst pattern with $-10 \% 5$ th order cos harmonic distortion . . . . . . . . . . . . . . . 150

A14 Demodulation and non-linearity of Null burst pattern with $10 \%$ th order cos harmonic distortion . . . . . . . . . . . . . . . 151

A15 Demodulation and non-linearity of Null burst pattern with $-10 \%$ th order sin harmonic distortion . . . . . . . . . . . . . . . . . 152

A16 Demodulation and non-linearity of Null burst pattern with $10 \%$ 5th order $\sin$ harmonic distortion . . . . . . . . . . . . . . . . 153 


\section{List of Tables}

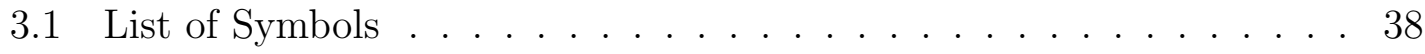

3.2 Identification Results . . . . . . . . . . . . . . . . . . 54

4.1 Identified Parameters of Linearized Model _ . . . . . . . . . . . . 80

5.1 Identification Results . . . . . . . . . . . . . . . . . 92 


\section{Nomenclature}

The following abbreviations are frequently used throughout this thesis:

ABS Air Bearing Surface

AD Areal Density

ADC Analog-to-Digital Converter

AFC Adaptive Feedforward Control

AFM Atomic Force Microscopy

AGC Automatic Gain Control

ASTC Advanced Storage Technology Consortium

BPM Bit Patterned Media

CWRRO Continuous WRRO

DUT Device under Test

FE $\quad$ Format Efficiency

FWHM Full Width at Half-Maximum

HAMR Heat-Assisted Magnetic Recording

HDD Hard Disk Drive

HDMR Heated-Dot Magnetic Recording

HMS Head-to-Media Spacing

ID Inner Diameter

IDC International Data Corporation

ILC Iterative Learning Control

KFCI Kilo Flux Change per Inch

MD Middle Diameter

MR Magnetoresistive

MTB Multi-Track Blending

MWW Magnetic Writer Width

NFT Near Field Transducer

NMS NFT Tip and Media Spacing 
NRRO Non-Repeatable Run-Out

OD Outer Diameter

OSA Over-Shoot Amplitude

OSD Over-Shoot Duration

OTRC Off-Track Read Capability

PC Personal Computer

PCB Printed Circuit Board

PES Position Error Signal

PLL Phase-Locked Loop

PMR Perpendicular Magnetic Recording

PSIM Planar Solid Immersion Mirror

RRO Repeatable Run-Out

SATA Serial Advanced Technology Attachment

SIL Solid Immersion Lens

SIM Solid Immersion Mirror

SMF Sync Mark Found

SMR Singled Magnetic Recording

SNR Signal-to-Noise Ratio

SSD Solid State Drive

SSM Servo Sync Mark

SUL Soft Underlayer

TDMR Two-Dimensional Magnetic Recording

TFC Thermal Fly-Height Control

TMR Track Misregistration

TRO Total Run-Out

TWRRO Traditional WRRO

VCM Voice Coil Motor

VGA Variable Gain Amplifier 
WRRO Wedge RRO 


\section{Chapter 1}

\section{Introduction}

\subsection{Background}

The hard disk drive (HDD) technology has gone through tremendous progress since its introduction back in the 1950s. When the personal computer (PC) era began in the 1980s, the HDD proliferated on PC as the low-cost high-capacity secondary storage device to store and retrieve the digital information. The HDD is a kind of magnetic storage device and magnetic materials are coated over the surface of the rigid disks. The data can be retained after the power is turned off. The HDD is also a very accurate and sophisticated mechanical system. One or more disks are rotating in the range of 5 to 15 kilorpm. The read and write heads are placed at the tips of the sliders, which are mounted on the arms. The heads are used to track the data tracks accurately. During read and write operation, the head must be flying very close to the disk surface with the clearance of less than $2 \mathrm{~nm}$ with the latest technology.

The first commercially available HDD was introduced in 1956 by IBM, which 


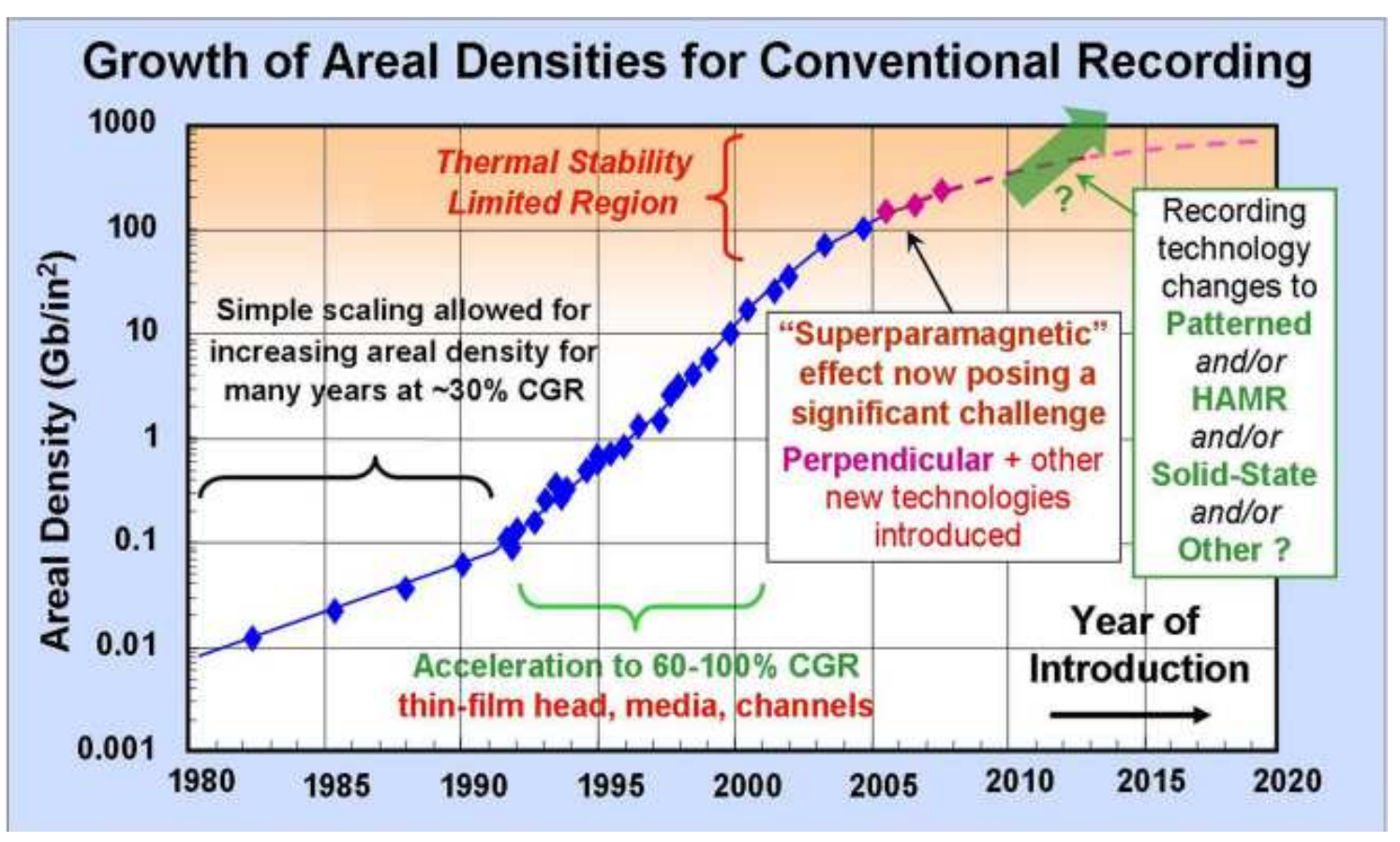

Figure 1.1: HDD areal density growth [2].

has the capacity of 3.75 megabyte [1]. Since then, the HDD storage capacity has been increasing by about 30\% every year on average. However, the rate of increase has dropped significantly to $10 \%$ from 2011 to 2015. As in 2014, the commercial HDDs areal density (AD) has reached 600 gigabyte/in ${ }^{2}$. In June 2015, HGST, a subsidiary of Western Digital, has shipped the world's first 10 terabyte HDDs. The overall growth of AD from 1980 is illustrated in Figure 1.1.

However, the HDD industry is facing a few major and tough challenges currently $[3,4]$. Firstly, the AD growth rate started to stall in 2012 when the perpendicular magnetic recording (PMR) reached its limit in so-called superparamagnetism [5]. Secondly, the HDD is losing its PC market share due to the competition from the solid-state drive (SSD) and the development of the cloud storage. Nowadays, more and more people choose to store their data remotely on the server resided in the data center. As a result, they can afford to buy an SSD with smaller storage capacity, but lightweight, faster access time and better energy efficiency. The future of HDD lies in the data center application, where bigger capacity and low cost are 


\section{Conventional Writes}

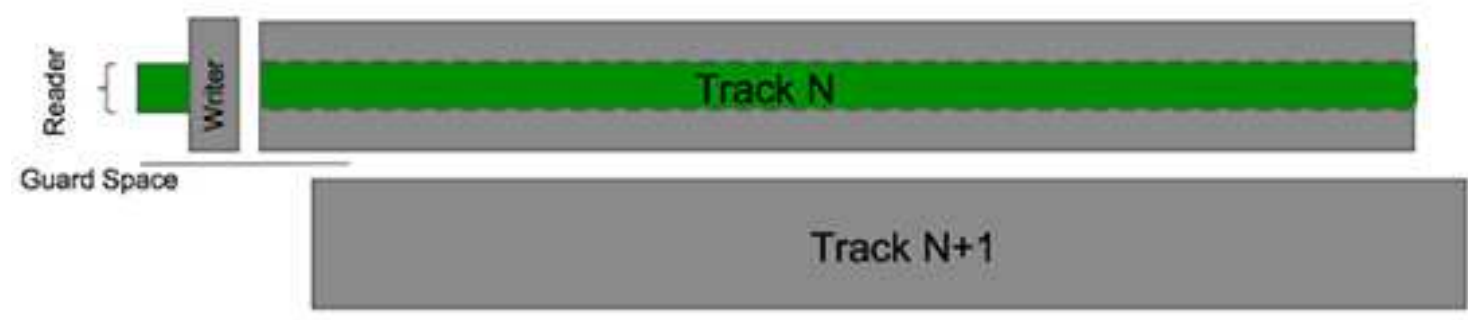

\section{SMR Writes}

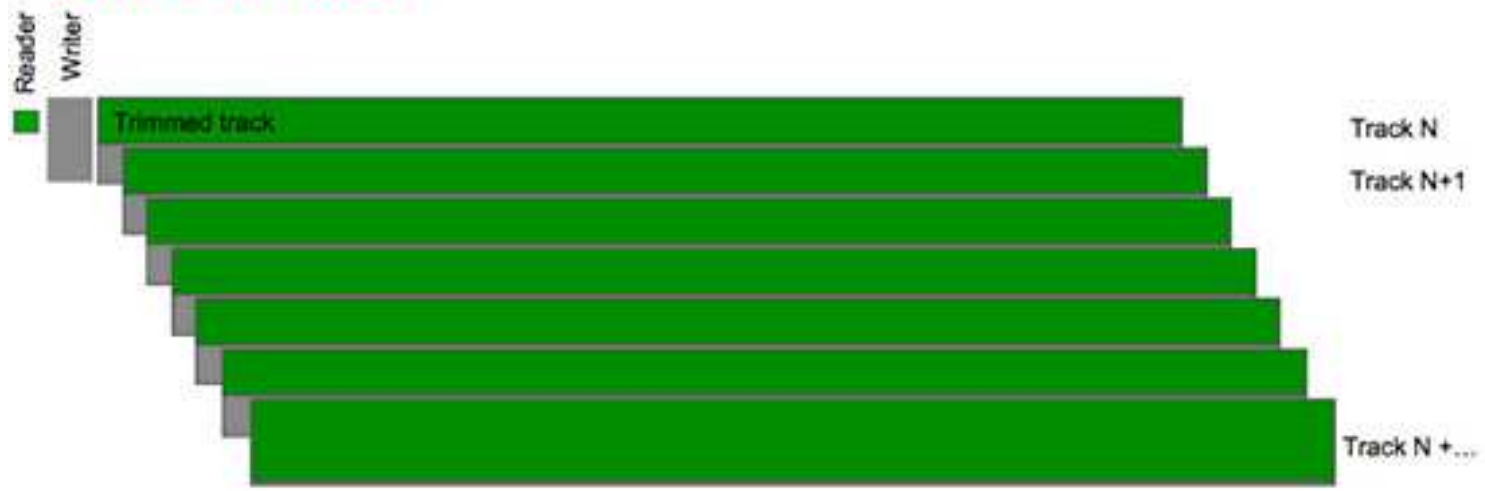

Figure 1.2: Concept of Shingled Magnetic Recording [6].

the dominating factors. The industry is striving to break the AD growth bottleneck by a few new technologies, such as shingled magnetic recording (SMR) [6-9], twodimensional magnetic recording (TDMR) [7,10-14], heat-assisted magnetic recording (HAMR) [5] and bit patterned media (BPM) [15].

\subsubsection{Shingled Magnetic Recording}

SMR is aimed to leverage on the current PMR head and media technology as much as possible. In conventional recording technology, data tracks are isolated with each other. A gap is left in between to avoid overwriting the adjacent tracks while writing a new one. As a result, the data track pitch is determined by the sum of the writer width as well as the gap. However, for SMR, the new data track will purposely overwrite a portion of the previous track and make each track narrower 


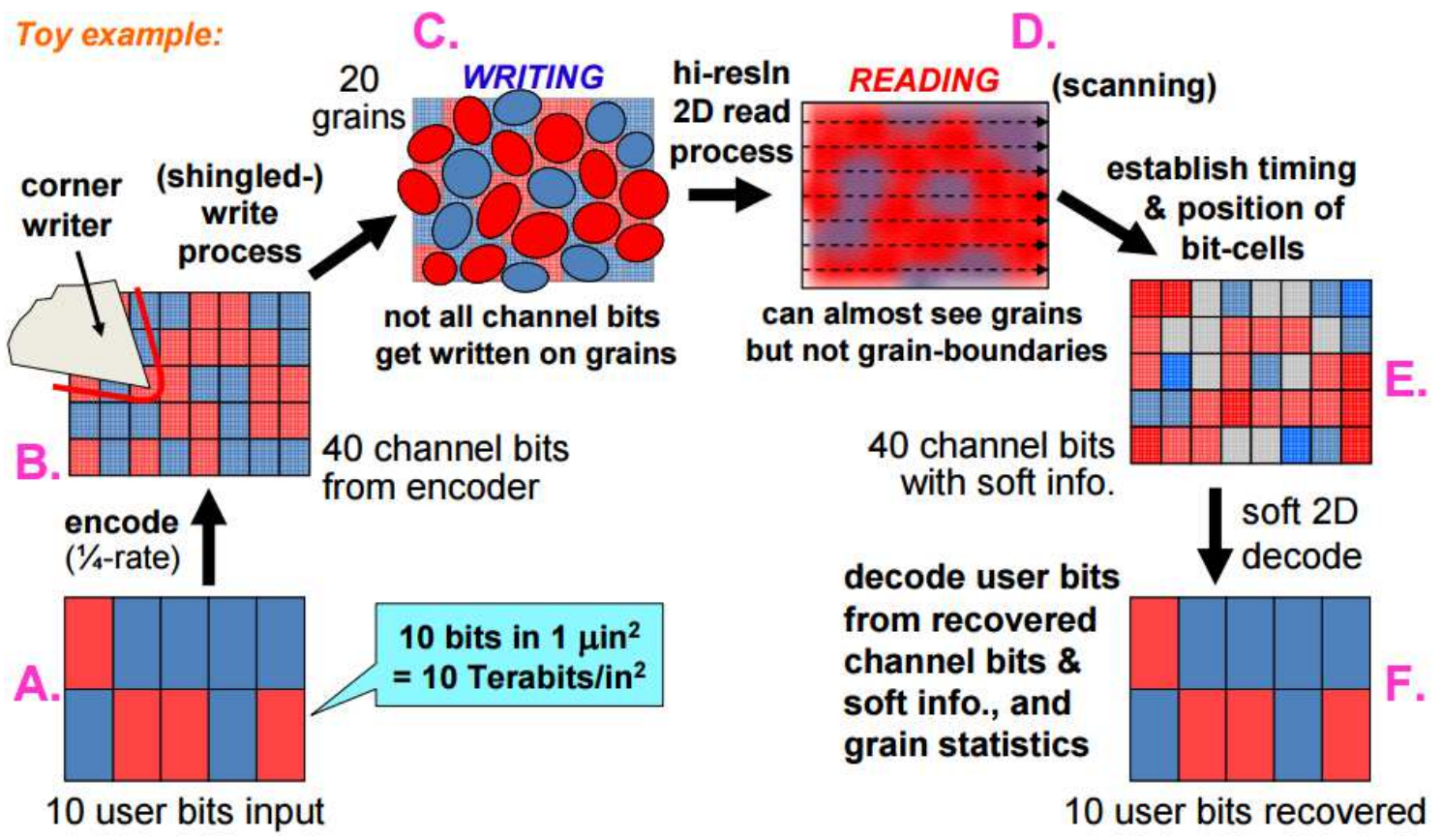

Figure 1.3: Concept of Two-Dimensional Magnetic Recording [7].

compared to the isolated tracks. The comparison between conventional writes and SMR writes is shown in Figure 1.2. Improvements of head design are required for good track edge writing and trimming, which is essential to the success of SMR.

\subsubsection{Two-Dimensional Magnetic Recording}

TDMR is firstly proposed to bring the AD toward 10 terabyte $/ \mathrm{in}^{2}[10]$ and its basic concept is shown in Figure 1.3. When track pitch and reader width become significantly smaller, the signal-to-noise ratio (SNR) will drop considerably. When the reader is away from the track center for a small distance, the SNR will become so low such that the data cannot be read back reliably. In order to improve the SNR, the multiple-reader array is proposed. Any two individual readers will have some portion overlapping with each other [10]. And the read back data will contain information for a multiple number of tracks. To extract the desired information from the very noisy data, a two-dimensional digital processing technique must be 


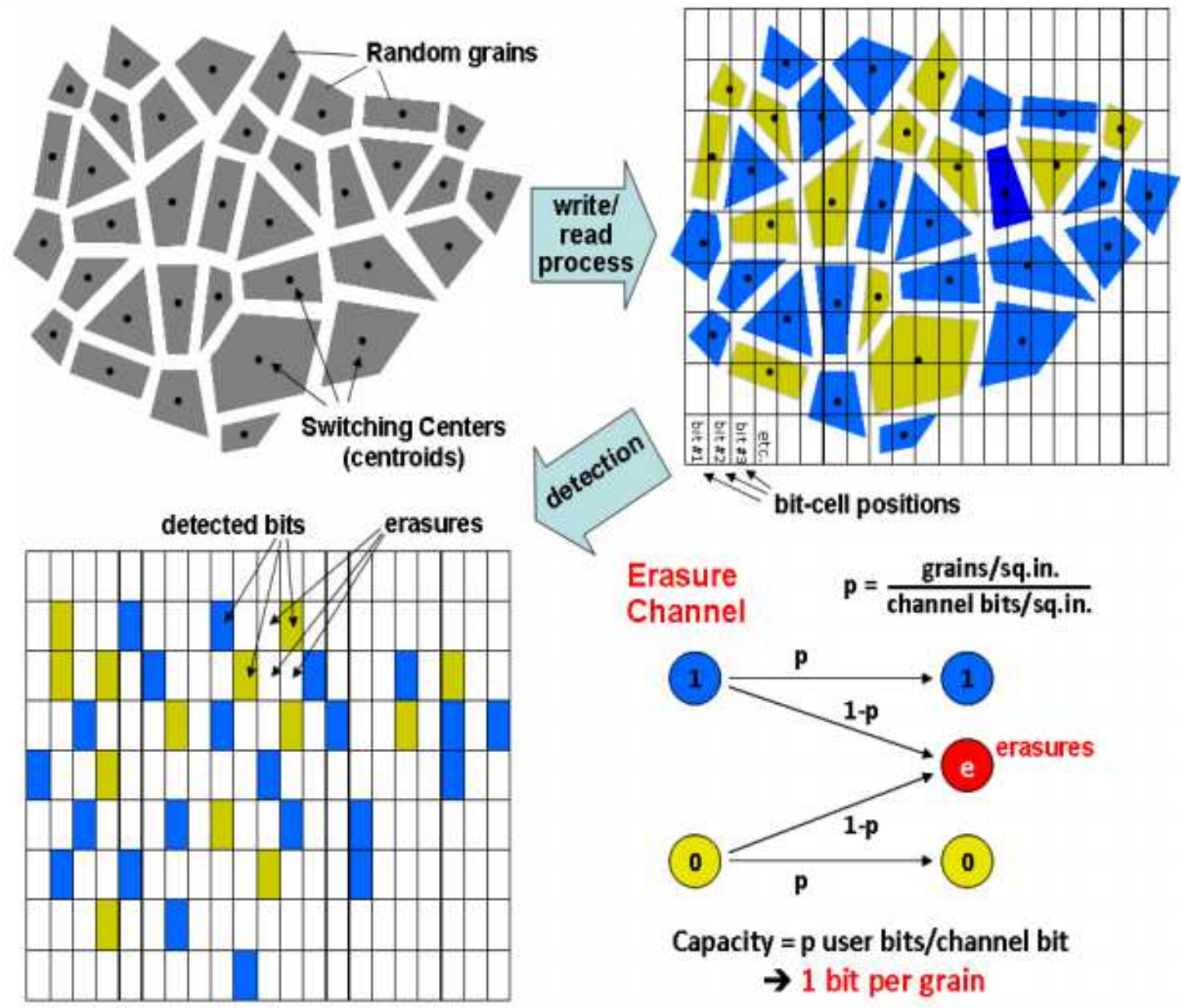

Figure 1.4: Illustration of Two-Dimensional signal processing for TDMR [7].

developed as illustrated in Figure 1.4. However, the recent research shows that the capacity gain is only about $6 \%$ from TDMR alone [14].

\subsubsection{Heat-Assisted Magnetic Recording}

HAMR is the new technology that all the HDD companies are focusing on. It is the most promising technology to bring the HDD AD to more than a few terabyte $/ \mathrm{in}^{2}$. Seagate announced that it will ship its first HAMR drive in around 2017 [16]. To avoid superparamagnetism (magnetization of nanoscale magnetic grain will flip direction due to the influence of temperature), the magnetic media is made 


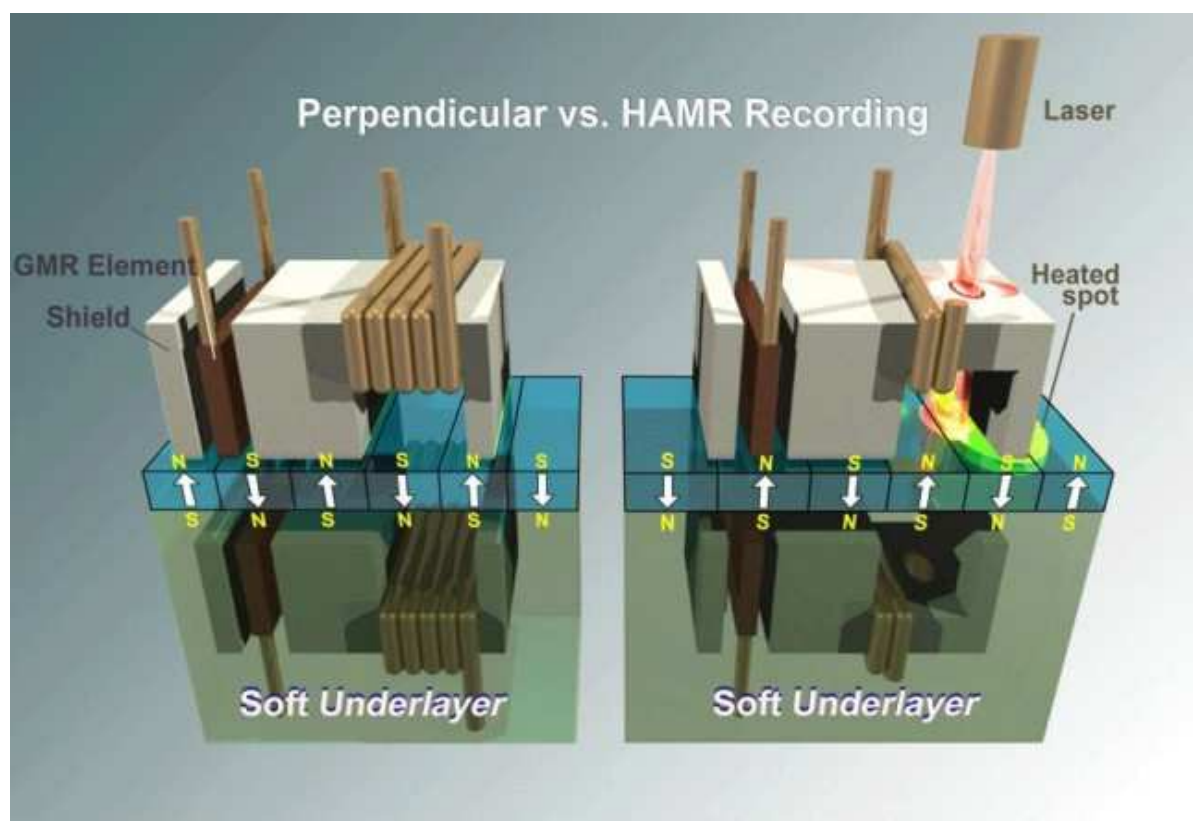

Figure 1.5: PMR vs. HAMR [6].

of the material of high coercivity, which will make it more difficult to flip the direction of magnetization. However, high coercivity also makes the data writing more difficult as well. The basic idea of HAMR is to fire a laser beam to heat up the magnetic grains through near field transducer (NFT), and reduce the coercivity of the magnetic material to facilitate the writing process. When the writing is finished, the media are cooled down and will go back to high coercivity state. And the recorded data will be retained with good stability. The main challenges of this technology are the life span of the head and the media design. Figure 1.5 makes a simple comparison between the PMR and HAMR to illustrate their major differences.

\subsubsection{Bit Patterned Media}

The basic idea of BPM is to discretize the media into magnetized islands, which are isolated by non-magnetic material. Each island will record one bit of data. And the isolation will improve the SNR by reducing cross-talk, erase band and adjacent track write [17]. The concept is illustrated in Figure 1.6. When work- 


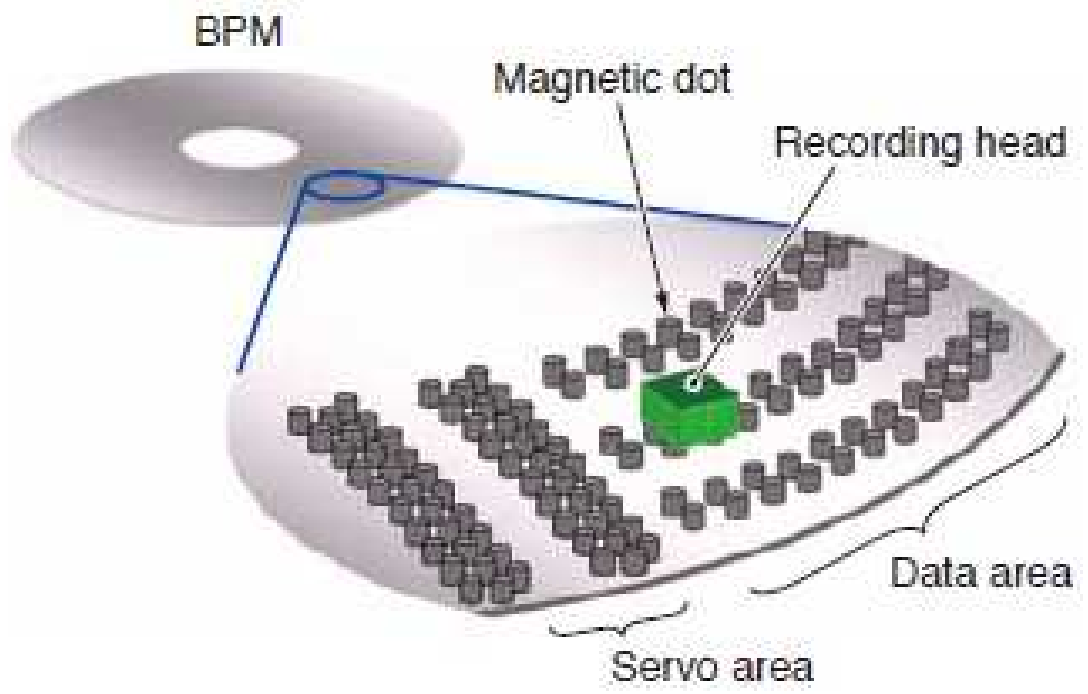

Figure 1.6: Concept of Bit Pattern Media [15].

ing together with HAMR, this is considered the ultimate technology for magnetic recording. However, BPM faces two great challenges. One is to develop the process to fabricate such media in a cost effective way. And the other is the writing timing synchronization with the physical bits in the media [17].

\subsubsection{HDD Technology Roadmap}

Above sections discussed the most viable technologies to increase the HDD AD further. The current AD is around 1 terabyte $/ i n^{2}$ as in 2016. The HDD technology roadmap by Advanced Storage Technology Consortium (ASTC) as shown in Figure 1.7 predicts that the $\mathrm{AD}$ will reach around 8 terabyte $/ i n^{2}$ by 2025 with the heated-dot magnetic recording (HDMR) which is a combination of BPM, HAMR and TDMR. 


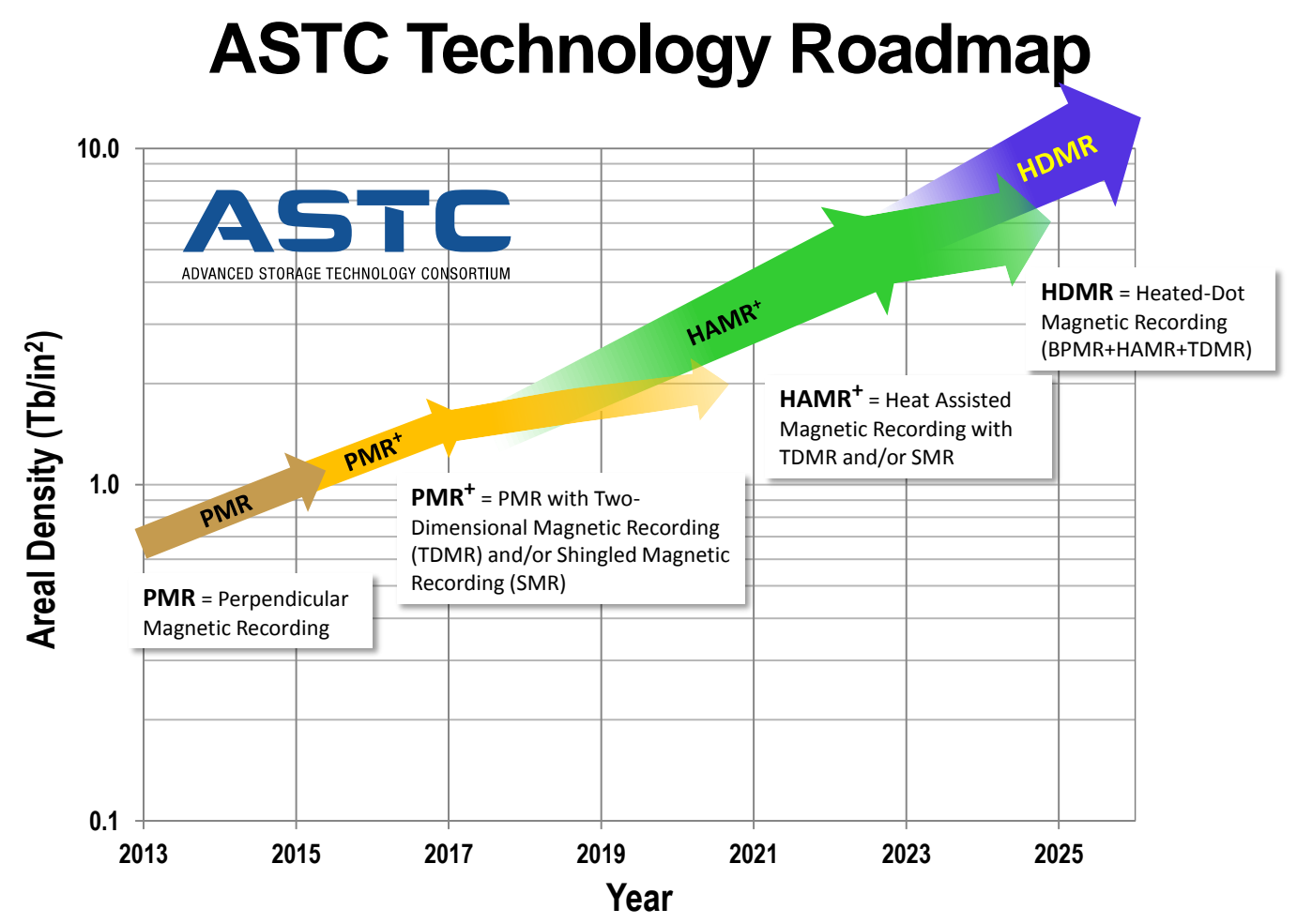

IUEMA

ASTC Proprietary

Figure 1.7: 2016 ASTC technology roadmap [18].

\subsection{Motivation}

Among all the aforementioned new technologies, HAMR is the most viable and promising technology, which is expected to bring the AD beyond a few terabyte $/ \mathrm{in}^{2}$ level and the data track width will be reduced to a few tens of nanometers. Researchers and engineers have done excellent jobs to make the read and write head follow the narrow data tracks, which are written in the high-speed rotating disks, with the help of excellent and reliable mechanical design, sophisticated controller design, embedded servo system, repeatable run-out (RRO) compensation scheme, and accurate head-to-media spacing control. However, narrower tracks impose more stringent and higher requirements on servo performance to follow the data tracks accurately. This motivates us to study how to further improve the servo or track- 


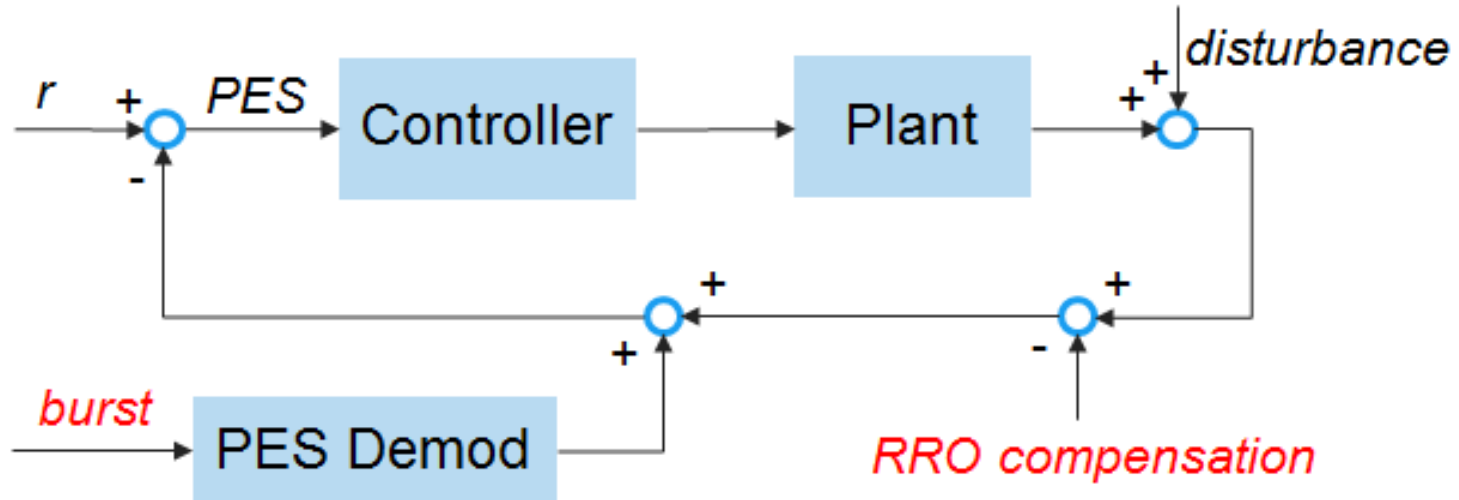

Figure 1.8: Block diagram of servo control system. The burst pattern and RRO compensation are the focuses of this thesis to improve the servo performance.

following performance to meet the requirements of the future high AD HAMR HDDs.

The simplified servo system block diagram is shown in Figure 1.8. In general, there are two major areas to work on in order to improve the servo performance of HDDs. The first area is to improve the mechanical design to increase the bandwidth of the plant, and reduce the mechanical vibration, disturbance, windage etc. With better plant, a higher bandwidth controller can be designed to attenuate the vibration more effectively. The second area is to improve the accuracy of the position error signal (PES) which was written in the manufacturing process that has all kinds of disturbances. PES is demodulated from the burst field in the servo sector. And RRO compensation values are learned and stored in the RRO field which is in the servo sector as well to reduce such error. The main purpose of RRO compensation is to cancel the repeatable disturbance to make the head follow the ideal circular tracks. This thesis focuses on the written-in quality of burst and RRO fields in the context of HAMR technology as shown in the red color of the block diagram. A new RRO compensation scheme called Continuous Wedge RRO (CWRRO) compensation scheme is also invented to improve the RRO correction effectiveness at off-track locations for off-track servo performance mitigation. In addition, it supports flexible drive capacity, which requires a flexible data track pitch [19] that traditional WRRO does not support. 


\section{$1.3 \quad$ Objectives}

With aforementioned motivations, the objectives of this research focus on the improvements of servo performance for HAMR HDDs by writing good quality burst and RRO fields with the help of HAMR writing process modeling. Further on, a new method is introduced to reduce burst non-linearity and the new CWRRO scheme for RRO compensation is invented to improve servo or track-following performance in high AD HAMR HDDs.

- The characteristics of the writing process for the HAMR is fundamentally different from the PMR technology. As a result, a better understanding of the HAMR writing process is needed to write good quality servo patterns such as burst field and RRO field. For HAMR, the media thermal profile is the major determining factor for the written-in signal quality. The media thermal profile is then determined by the thermal power delivered to the media and the thermal properties of the media stack. The available thermal power is then determined by the NMS and input laser power. However, the NMS keeps changing due to various protrusions induced by the heat sources related to the laser. The worst thing is that instantaneous NMS is not measurable due to NFT's tiny size. Our first objective is to construct and identify a dynamic model for the HAMR writing process so that the various dynamic responses involved in the writing process can be better understood and characterized.

- Our ultimate goal is to write the high-quality servo burst pattern and RRO compensation fields using the HAMR technology. The identified dynamic model for HAMR writing process enables us to optimize the NMS and input laser power profile so that the high-quality burst patterns and RRO fields can be written without shortening the lifetime of the NFT unnecessarily and losing of the format efficiency (FE). 
- For HDDs, PES is the only feedback signal to indicate the current head position in the radial direction. For embedded servo system, PES is demodulated from the burst pattern which is written in the field dedicated to the servo control called servo sectors or wedges. Burst demodulation is non-linear in nature. Although non-linearity correction techniques have been developed, but nonlinearity residues still degrades the track-following performance. Our goal is to improve the burst demodulation non-linearity and consistency for HAMR drive. This will improve the accuracy of the PES signal and track-following performance in the end.

- Due to the elimination of the space between adjacent tracks by SMR technology and the decrease of track width, the current RRO compensation scheme and technology cannot work properly due to the limited off-track read capability (OTRC) [20,21]. A new RRO compensation scheme will be developed, which has good OTRC and acceptable track misregistration (TMR) [22] performance at any off-track locations for high AD HAMR HDDs.

\subsection{Contributions}

The major contributions of this thesis are shown below, which are aligned with the objectives listed in the above section:

- In order to understand the HAMR writing process and control the written-in signal quality, a dynamic model for the HAMR writing process is constructed and identified. The model inputs are input laser power and fly-height control, and the outputs are the signal magnitude and NMS change. The model consists a few building blocks such as NFT slow and fast protrusions, energy transfer efficiency function, heat transient process, and thermal power to signal magnitude conversion. Using this model, the real time NMS change and 
written-in signal magnitude during the writing process under different input conditions can be estimated. The model construction and parameter identification processes are discussed in detail in Chapter 3, which is based on our paper [23]. To our knowledge, this is the first paper that reports an HAMR writing process dynamic model.

- The dynamic writing process model provides a few important applications. By using this model, the NMS during the whole writing process can be optimized [24]. When the NMS is kept constant, one of the sources that affects the consistency of the written-in signal quality is eliminated. As a result, the burst pattern with minimum non-linearity and good quality RRO fields can be written. Additionally, by minimizing the NMS variation, the input laser power to achieve the same SNR can be reduced. Less input laser power can reduce the temperature of NFT and increase its lifetime. To achieve this, a linearised version of this model is used to derive the optimal input laser current overshoot profiles to compensate for the transient process of the HAMR writing process. It has been proved that two sets of laser overshoot current profiles are required to compensate for the transient process almost completely. By compensating for the transient process, the FE overhead while writing the very short length data such as the RRO field can be reduced. The details of these applications are summarized in Chapter 4.

- To characterize the shape of the burst profile, two parameters are proposed such as flatness [25] and newly created asymmetry. A near optimal burst profile is identified, which can achieve PES error of less than $1 \%$ track width (1\%trk) through the simulation. Such burst profile has 0 asymmetries and a flatness around 0.82 . The burst non-linearity can be minimized by controlling the ratio between reader width and track width to about $68 \%$ for the particular head tested. These works are discussed in Chapter 5 . 
- A revolutionary new scheme is developed, which is called Continuous Wedge RRO (CWRRO) compensation scheme, to replace the current RRO compensation scheme $[21,26,27]$. CWRRO scheme can provide RRO compensation for any off-track locations rather than the track center only by current RRO compensation scheme. A method called multi-track blending (MTB) is invented to improve TMR performance of the basic CWRRO scheme. The basic CWRRO scheme can achieve a worst case TMR performance that is equivalent to the current scheme at $10 \%$ trk off-track from the track center. By using MTB, the worst case TMR performance is improved from $10 \%$ trk off-track to $7 \%$ trk. All the details are summarized in Chapter 6.

\subsection{Organization of the Thesis}

The rest of the thesis is organized as follows:

Chapter 2 gives some background on the HDD servo control system including the embedded servo, servo wedge structure, burst pattern and its demodulation, and RRO compensation field as well. In the remaining of this chapter, the HAMR technology fundamentals are explained for basic understanding.

In Chapter 3, the details are shown on how to construct the dynamic model for HAMR writing process and its identification procedures. It can be considered as a "Grey-Box" system parameter estimation problem. At the end of the chapter, the accuracy of the model is verified by comparing the model output and real drive measurement under various input conditions.

Chapter 4 discusses two important applications of the HAMR writing process model. One is the NMS optimization and control for different writing conditions. The other application is to find optimized input laser current profile to compensate 
for the writing transient responses.

Chapter 5 focuses on how to characterize the shape of the burst profile and find out the desired burst profile, which will produce minimum PES demodulation error. It is also presented on how to reduce the non-linearity of the written-in burst pattern.

In Chapter 6, a revolutionary CWRRO scheme is discussed in details. The CWRRO can provide sufficient OTRC with reasonable TMR degradation. The MTB, which is used to enhance the performance of the basic CWRRO scheme, is discussed in detail as well.

In Chapter 7, conclusions and recommendations for future research works are presented. 


\section{Chapter 2}

\section{HDD Servo Control System and HAMR Technology}

\subsection{HDD Servo Control System}

\subsubsection{Introduction}

The hard disk drive is a very accurate and sophisticated mechanical device. It comprises multiple disks and heads connected to a distal end of an actuator arm, which rotates about a pivot by a voice coil motor (VCM) to position the head radially over the disk. The disk rotates at the constant speed of 5 to $15 \mathrm{krpm}$ through the spindle motor and comprises a plurality of radially spaced, concentric tracks for recording user data sectors and servo sectors. Each servo sector comprises head positioning information, which is read by the head and processed by a servo control system to control the actuator arm as it seeks from track to track. Most of the hard disk drives today implement dual-stage actuators $[28,29]$ to increase the 


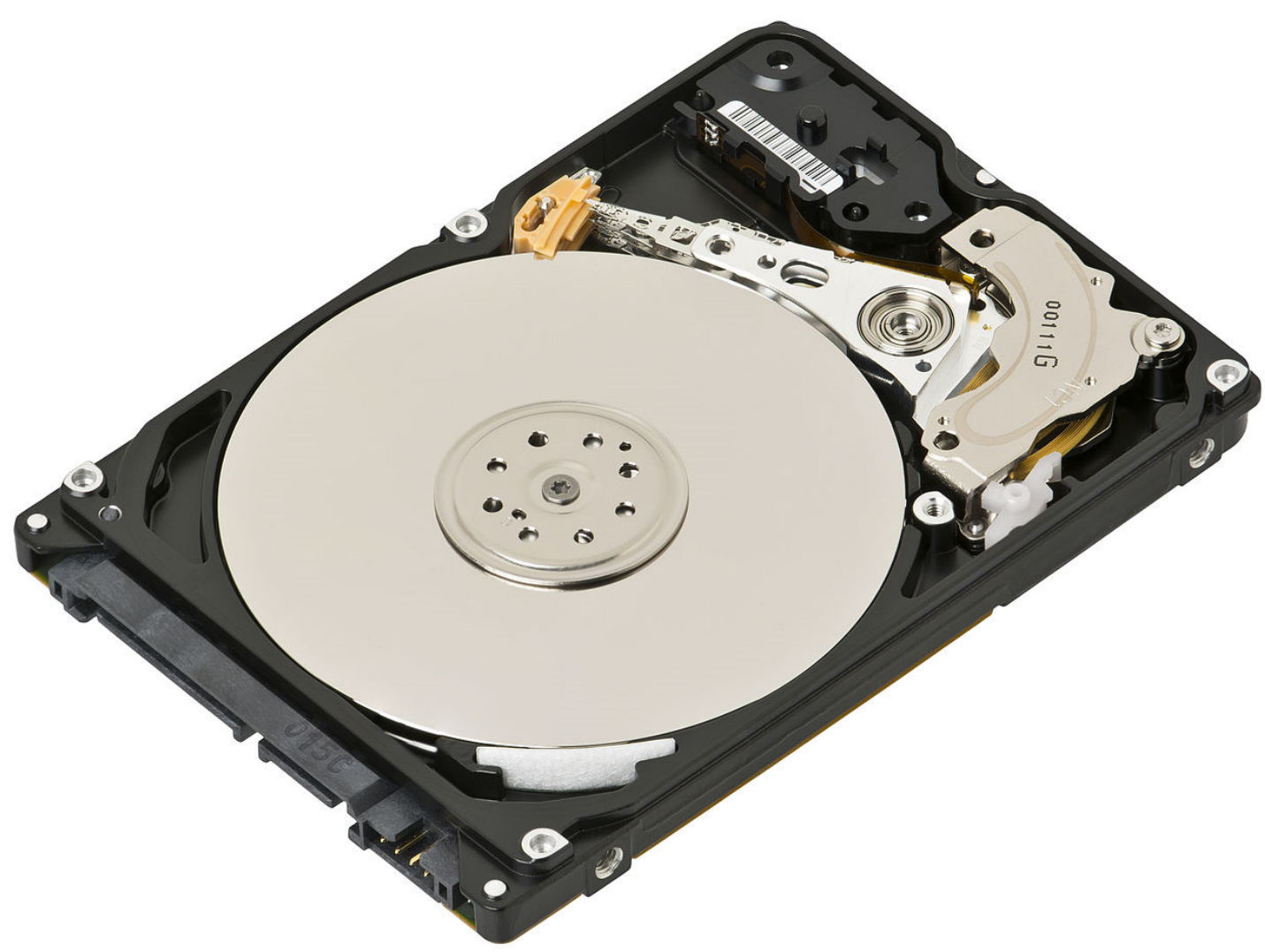

Figure 2.1: Internal structure of 2.5 inch 500 gigabyte Western Digital Scorpio Blue hard drive.

bandwidth of the controller system and improve the track-following performance. The first stage is the traditional VCM for the coarse movement and the second stage is implemented by piezoelectric actuator for the fine movement of the head. The internal structure of an 2.5 inch HDD is shown in Figure 2.1 and the head stack assembly is shown in Figure 2.2.

\subsubsection{Servo Sector and Position Error Signal (PES)}

The position error signal (PES) is the only feedback signal available for the closed-loop servo control to position the read head and write head. The PES is generated using the burst pattern written in the servo sector. For modern HDDs, the 


\section{Head Stack Assembly (HSA)}

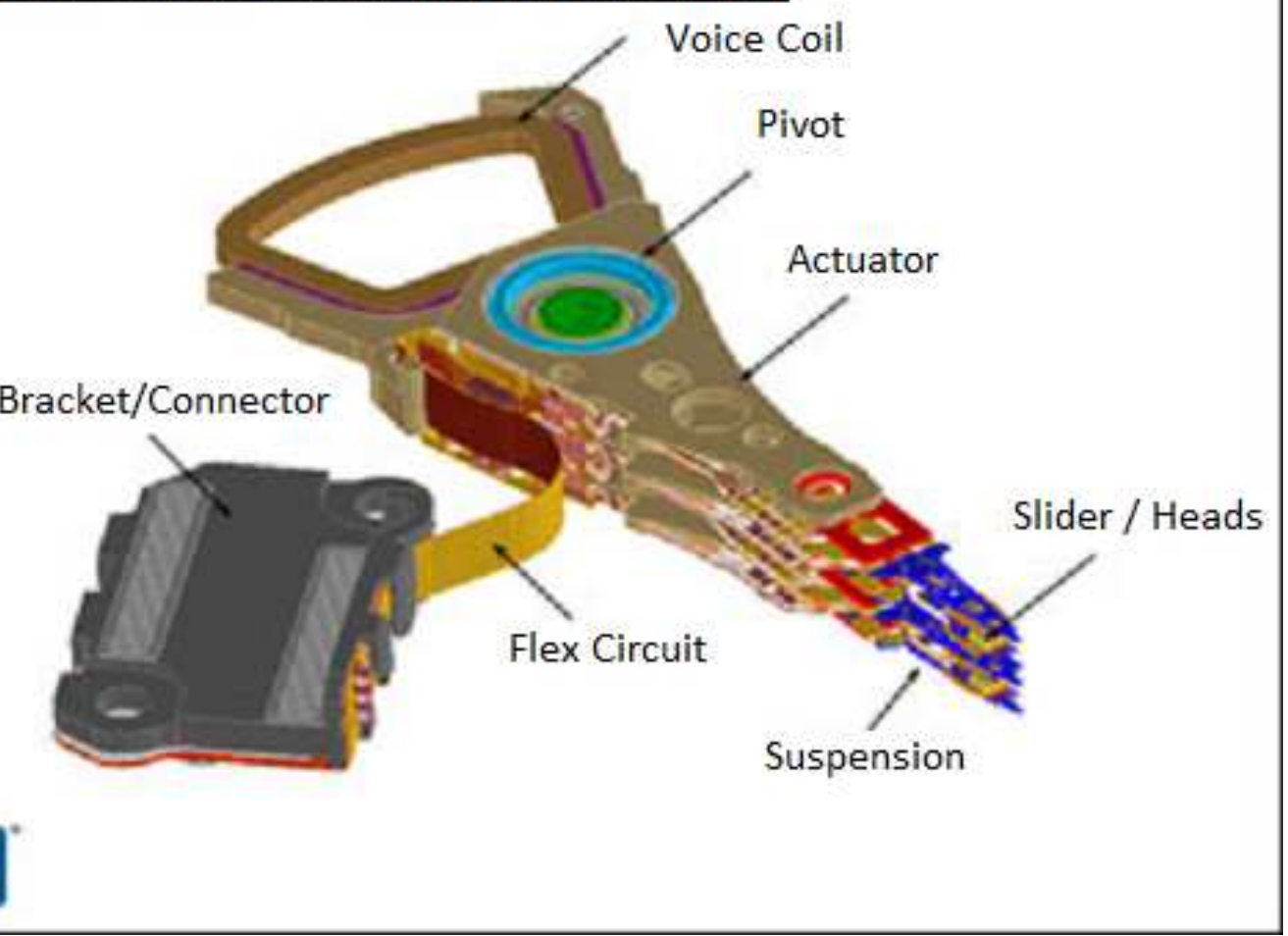

Figure 2.2: Head stack assembly.

embedded servo sector strategy $[30,31]$ is used. For embedded servo, the servo sectors are evenly spaced and interleaved with the data sectors. Servo sector is also called servo wedge and each servo sector contains at least 5 fields in one way or another, namely auto gain control (AGC), servo sync mark (SSM), servo sector identification (SID), the track identification (TID) and burst pattern. AGC field is used for the phase-locked loop (PLL) to synchronize the read back signal to the system clock. It is also used to adjust variable gain amplifier (VGA)'s gain to achieve the desired signal magnitude, which will be feed into the input of an analog-to-digital converter (ADC). SSM is a field with a selected pattern to have lowest detection error rate. Once this field is detected, hardware will issue sync mark found (SMF) signal which will be used as 0 time stamp for the current servo sector. SID stores the current servo sector identification and is normally in the range of a few hundred sectors. 


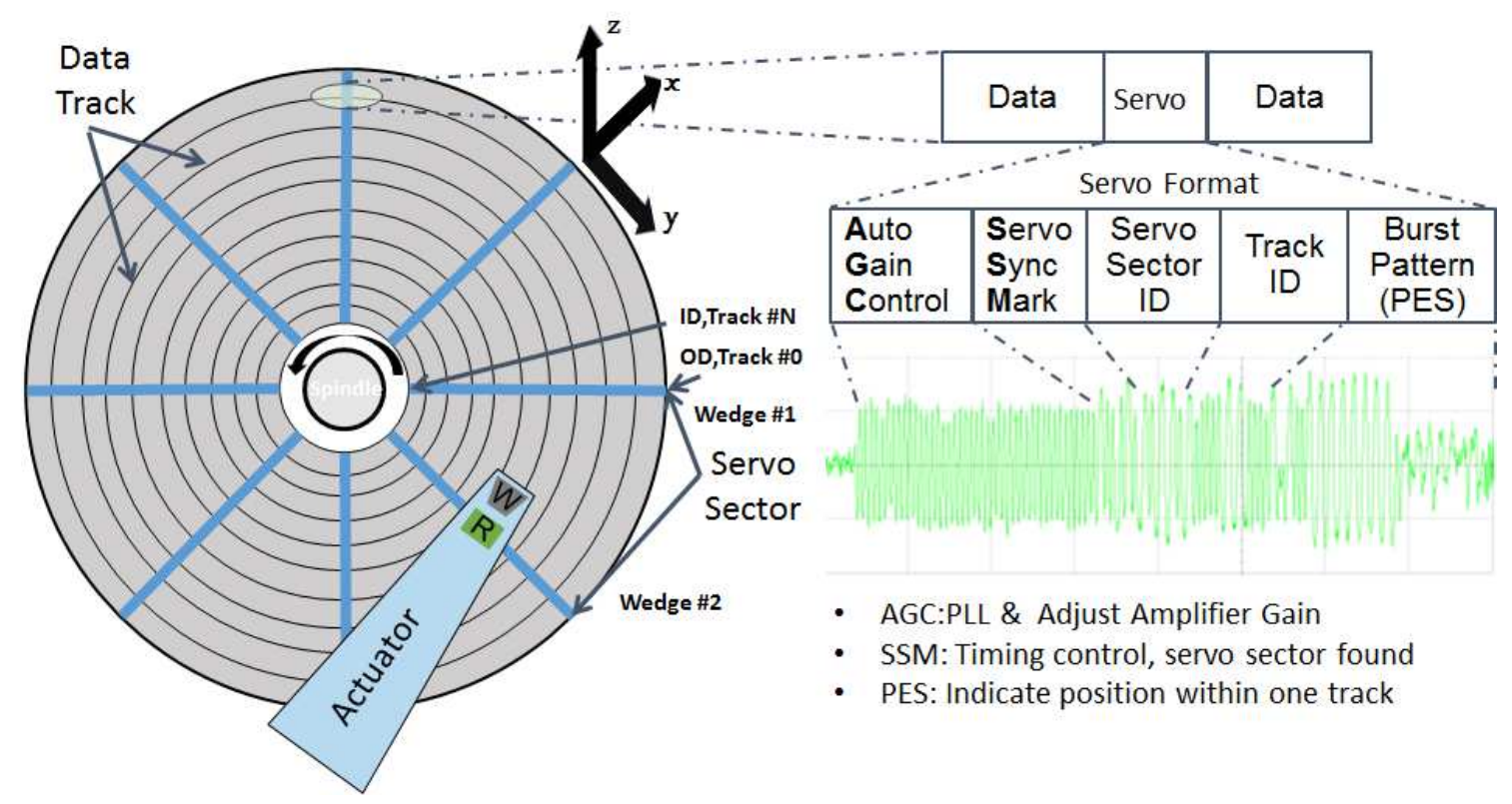

Figure 2.3: Embedded servo and servo sector/wedge.

TID indicates the servo track number. Burst pattern is a specially designed pattern to generate the PES signal, which will tell the head location within a track. Both TID and burst pattern work together to generate the final PES signal. TID tells the current track number that the head is on. Burst pattern signal is demodulated to indicate the off-track location away from the current track center $[32,33]$.

In general, servo sectors act as the ruler for the whole disk. More specifically, the SID is used to tell the head position in the down-track direction (Y direction shown in Figure 2.3) as the disk is rotating, and TID and burst pattern is used to indicate the head position in radial or cross-track $(\mathrm{X})$ direction. Some servo sectors may have an optional field called fly height field. This field can be used to measure the flying height of the head with respect to the media surface in vertical (Z) direction. 


\subsubsection{Track Mis-registration (TMR)}

The ultimate goal of the servo control system is to let the head precisely follow the desired data track. A common way to measure the track-following performance of the servo control system in HDD industry is to measure the track mis-registration (TMR) [34]. For simplicity, TMR is just some number statistically extracted from the PES of multi-revolution measurements. There are two parts in TMR: total run-out (TRO) and other stuff [34]. For simplicity, only TRO is discussed in the following. TRO consists of repeatable run-out (RRO) and non-repeatable run-out (NRRO). For example, if there are 300 servo sectors per revolution and PES is measured for consecutive 10 revolutions, there will be total 3000 PES values collected. The $3 \sigma$ value of the collected data is computed and converted to the percentage of track width (\%trk). This value is then called TRO in \%trk. The PES value for each servo sector at different revolution can also be averaged to get averaged PES value for each servo sector. The $3 \sigma$ value of the averaged value is then calculated and called RRO. If the original PES values are subtracted from the averaged values for each servo sector for all revolutions, the $3 \sigma$ of residual values is called NRRO. The RRO is mainly due to the disturbance and noise during the servo writing process where the servo sector is written, as well as disk eccentricity and spindle motor vibration. However, the NRRO is mainly due to the mechanical disturbance, measurement noise and electronic noise. People can also calculate and analysis the frequency spectrum of TMR from the frequency domain.

\subsubsection{Wedge Repeatable Run-out (WRRO) Compensation}

Due to the existence of RRO, the head track-following trajectory is not an ideal circle as desired, but the irregular shape as illustrated in Figure 2.4. RRO is mainly due to disk eccentricities, the imperfection of spindle motor assembly and magnetic 


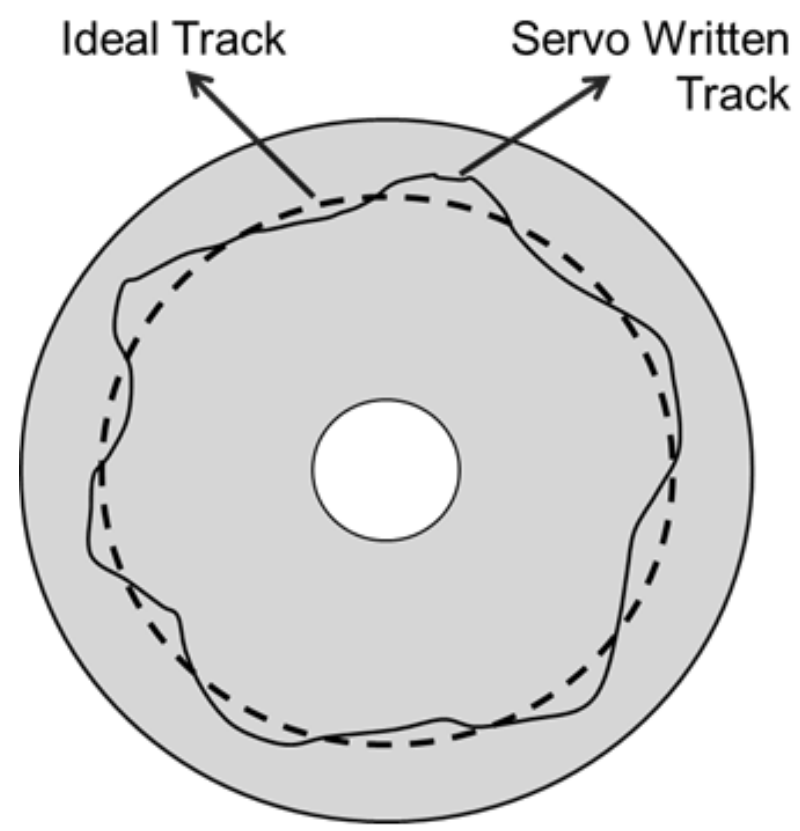

Figure 2.4: Servo written track and repeatable run-out (RRO).

media, and the disturbance and noise during the servo writing process. If the RRO of the adjacent tracks is in the opposite direction, it is more likely to over-write the adjacent track and damage the recorded data or degrade the signal quality during the reading process. Since the RRO will greatly degrade the servo performance of the HDD, many methods have been proposed to deal with the RRO [35-53].

The RRO are normally canceled in two ways. The first one is to "follow" the RRO [35-39]. One such method, called adaptive feedforward control (AFC) as shown in Figure 2.5, tries to make the head to follow the RRO by feedforward the online learned RRO to the plant. This method works only for low-frequency harmonics which are normally coherent from track to track. The advantage of this method is that it does not occupy the disk space since the RRO is learned in real time or online learning. However, the disadvantage is that it is computationally intensive, especially when the number of harmonics to be compensated is large.

The second way for RRO compensation is to "anti-follow" the RRO [40-46,53], which subtracts the learned RRO values from the PES so that the head trajectory 


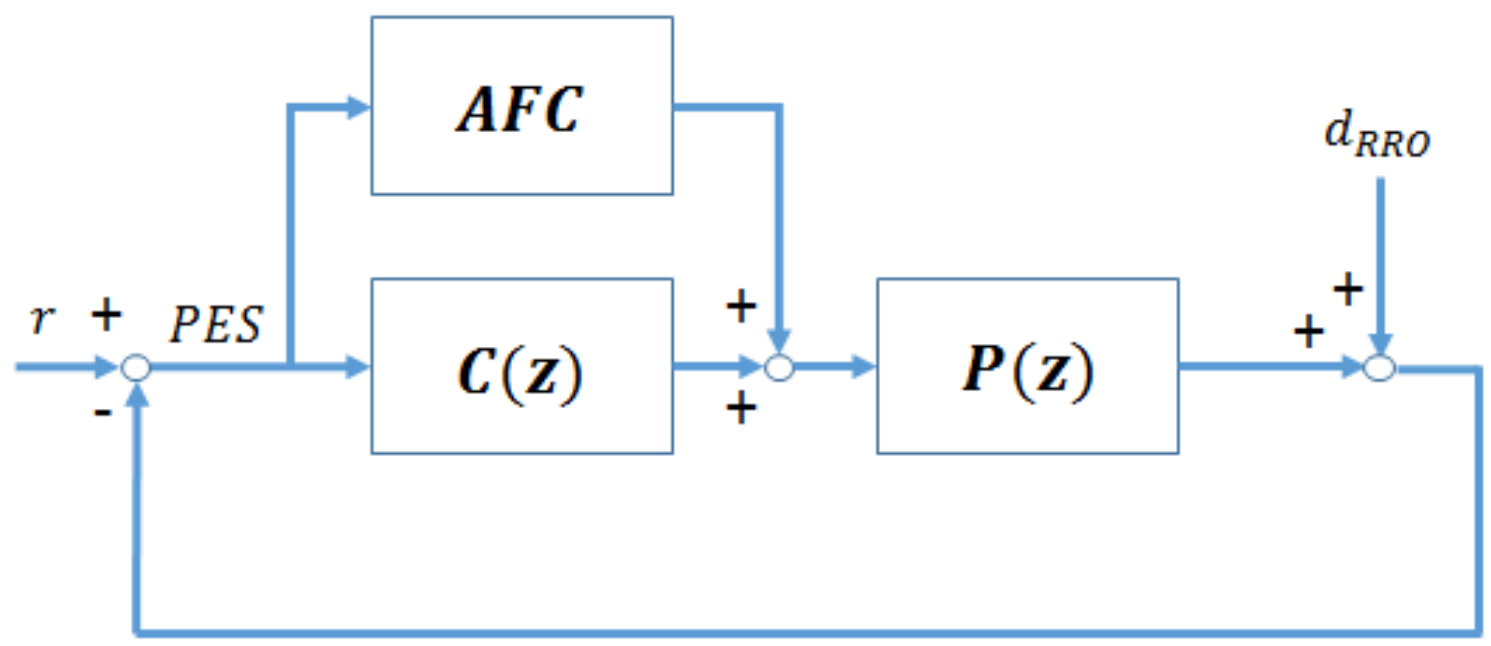

Figure 2.5: Block diagram for RRO compensation using adaptive feedforward control (AFC).

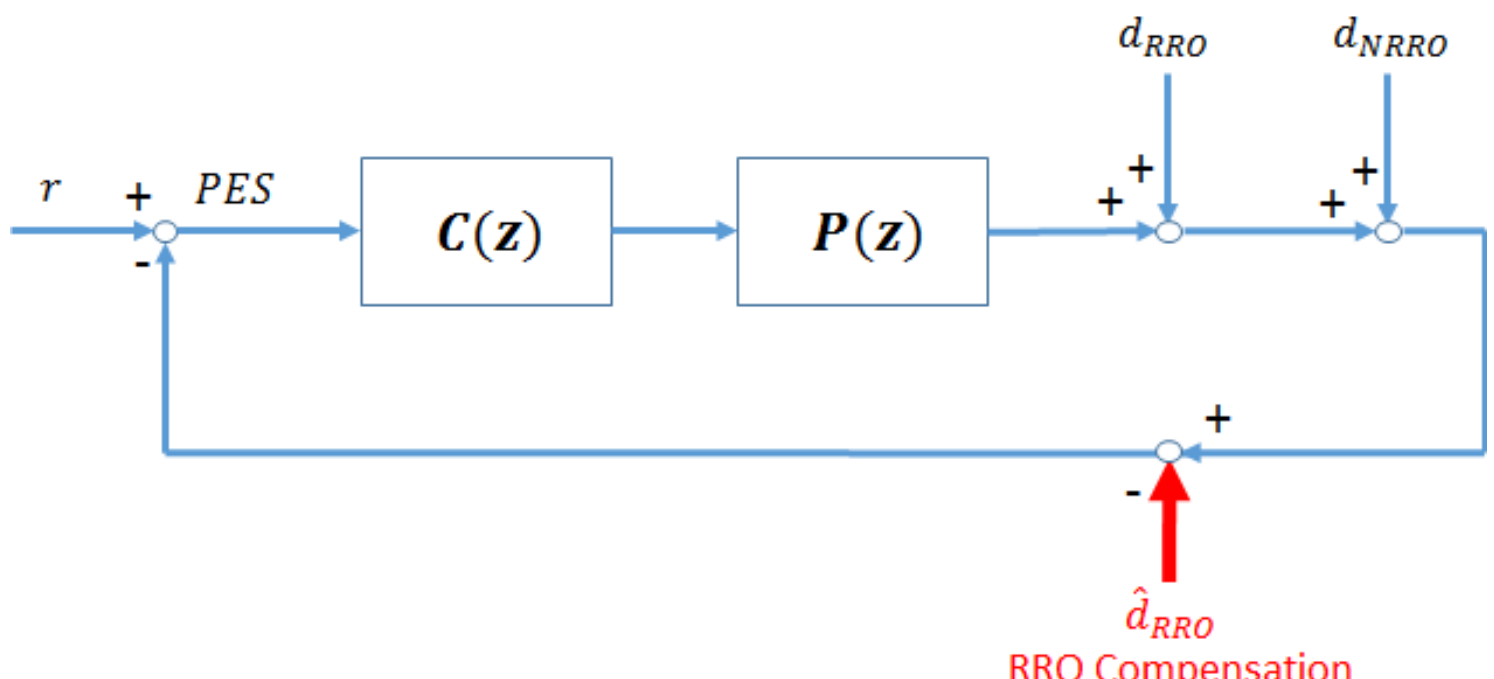

Figure 2.6: Block diagram for RRO compensation.

will follow the ideal circular track. The block diagram, which is shown in Figure 2.6, illustrates its basic concept. This method works best for non-coherent and highfrequency components. The critical part of this method is about how to learn the RRO accurately and efficiently. The basic idea of the learning process is to set the reference signal $r$ to 0 and measure the $P E S_{R R O}$ as shown in Figure 2.7. The RRO compensation values are then derived in (2.1). The transfer function $\frac{1}{1+P(z) C(z)}$ 


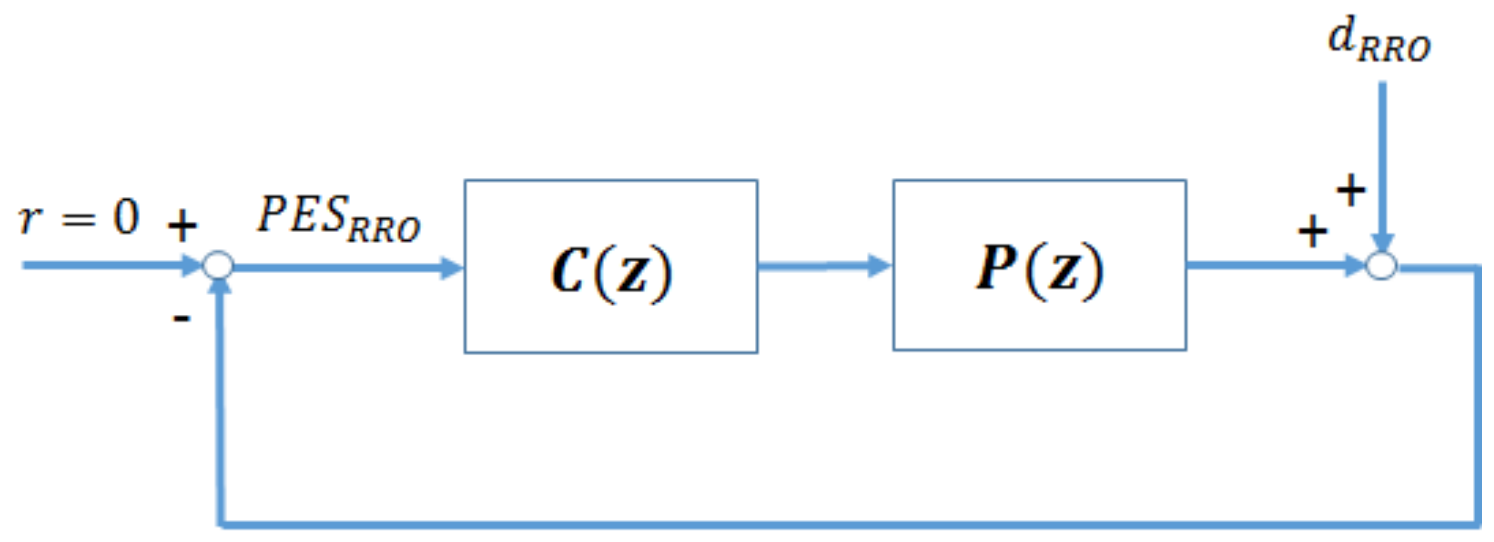

Figure 2.7: Block diagram for RRO compensation value learning.

is the error transfer function or sensitivity function. For this method, the RRO values are learned for each track and stored in the RRO field, which normally resides after the burst pattern in servo sectors $[43,54]$. Since all the RRO need to be stored on the disk, it will reduce the format efficiency since less space is available for user data storage. The advantage of this method is that there is no computation involved once the RRO learning and recording process is finished. In current HDDs, "follow" and "anti-follow" methods are designed to deal with different frequency harmonics. However, the two methods can be employed simultaneously at certain frequencies.

$$
\hat{d_{R R O}}=P E S_{R R O}=\frac{d_{R R O}(z)}{1+P(z) C(z)} .
$$

\subsection{HAMR Technology}

The global annual data growth rate of $40 \%$ is projected by the International Data Corporation (IDC). In order to meet the data storage requirements, the AD of HDD must be increased further while maintaining the low cost. However, the AD growth rate of current PMR HDD is around $10 \%$ only. The ultimate AD for PMR technology is around 1 terabyte $/ \mathrm{in}^{2}$ due to the superparamagnetic limit $[5,55]$. In order to retain the stored data for more than 10 years, the thermal stability of the 
magnetic grains must be maintained by following the relationship governed by (2.2).

$$
\frac{K_{u} V}{k_{B} T} \geqslant 70
$$

as shown in [5]

The $k_{B}$ is the Boltzmann constant, $T$ is the temperature in Kelvin. When the $\mathrm{AD}$ is increased, the magnetic grain volume $V$ must be reduced to keep the number of grains $N$ per bit area high enough to sustain enough SNR [56]. In order to maintain the relationship in (2.2), the uniaxial anisotropy energy density of the magnetic material $K_{u}$ must be increased to restore the thermal stability. However, higher $K_{u}$ media needs a higher magnetic field to write data onto such media. But the highest writing magnetic field is limited by the material available for making the head. In order to break the limit of PMR, HAMR is developed to increase the $\mathrm{AD}$ further by introducing one more freedom to the design, which is temperature. Seagate had demonstrated HAMR drive with AD of 1 terabyte/in ${ }^{2}$ [57] and 1.4 terabyte $/ \mathrm{in}^{2}$ [58] respectively in the lab. In the subsequent sections, the principle of HAMR technologies will be briefly discussed followed by the two most important elements for HAMR, which are media and light delivery system.

\subsubsection{Principle of HAMR}

The basic idea of HAMR is to heat up the media temporarily during the recording process so that the media coercivity can be lowered below available magnetic write field generated from the head, such as the PMR head. When the media temperature is elevated to the Curie temperature $\left(T_{c}\right)$, its saturation magnetization $\left(M_{s}\right)$, magnetic anisotropy $\left(K_{u}\right)$ and coercivity $\left(H_{c}\right)$ will drop to nearly zero [59]. The media will then cool down very rapidly while the writing magnetic field is applied. If the writing magnetic field is bigger than the local demagnetization field, 


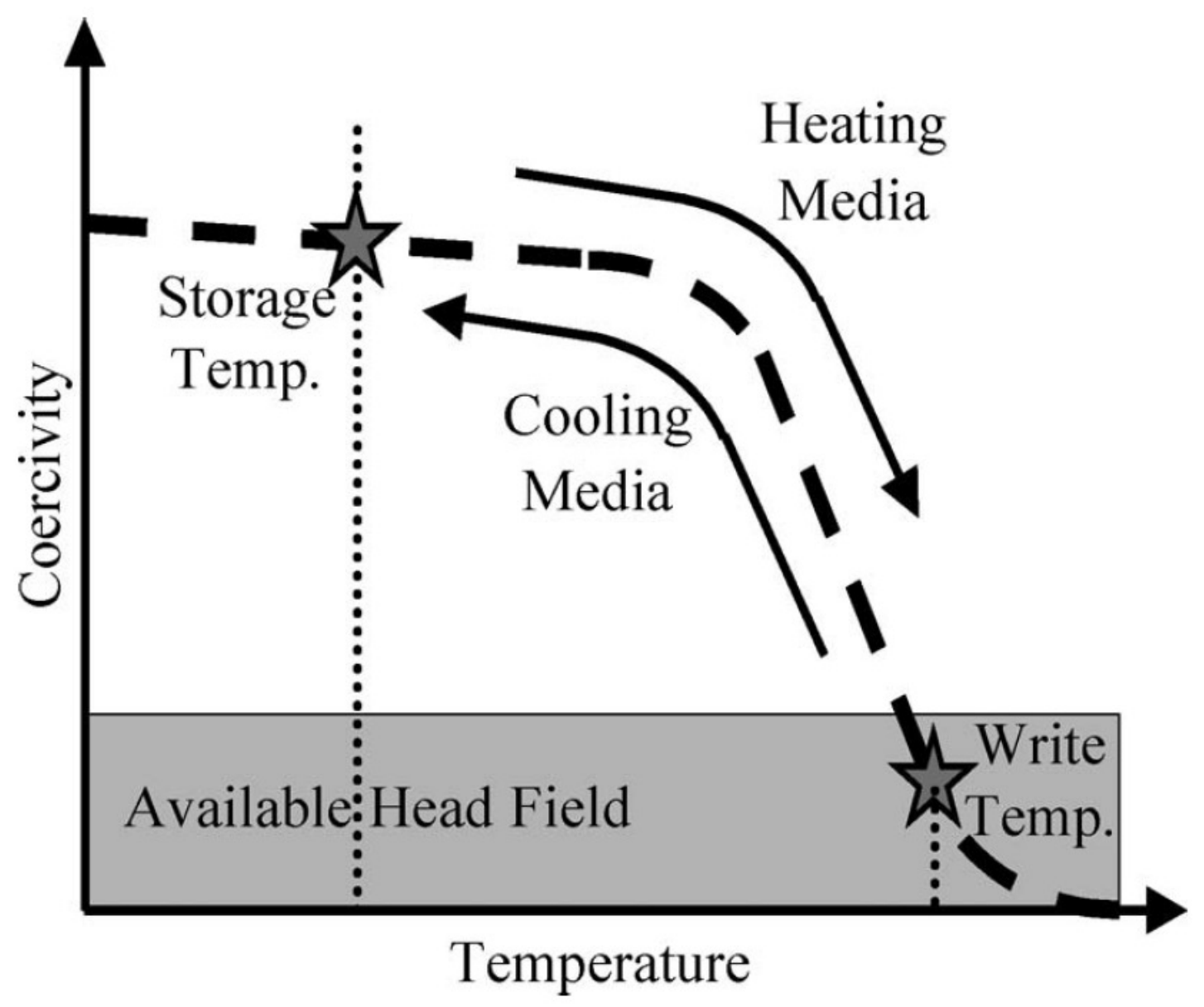

Figure 2.8: Principle of HAMR technology [5].

the orientation of the media grain magnetization will follow the writing field and the data will be recorded in the media. The whole idea is illustrated in Figure 2.8 [5]. Once the data is written in, the signal read-back method is the same as the PMR by using the magnetoresistive reader element.

The introduction of temperature as one more freedom in the writing process enables HAMR to produce very high effective magnetic writing field gradient without any improvement from current magnetic head design as shown in (2.3) [5].

$$
\frac{d H_{\text {write }}}{d x} \sim \frac{H_{k}}{d T} \cdot \frac{d T}{d x}
$$

The first item on the right-hand side of the equation is the slope of the media 


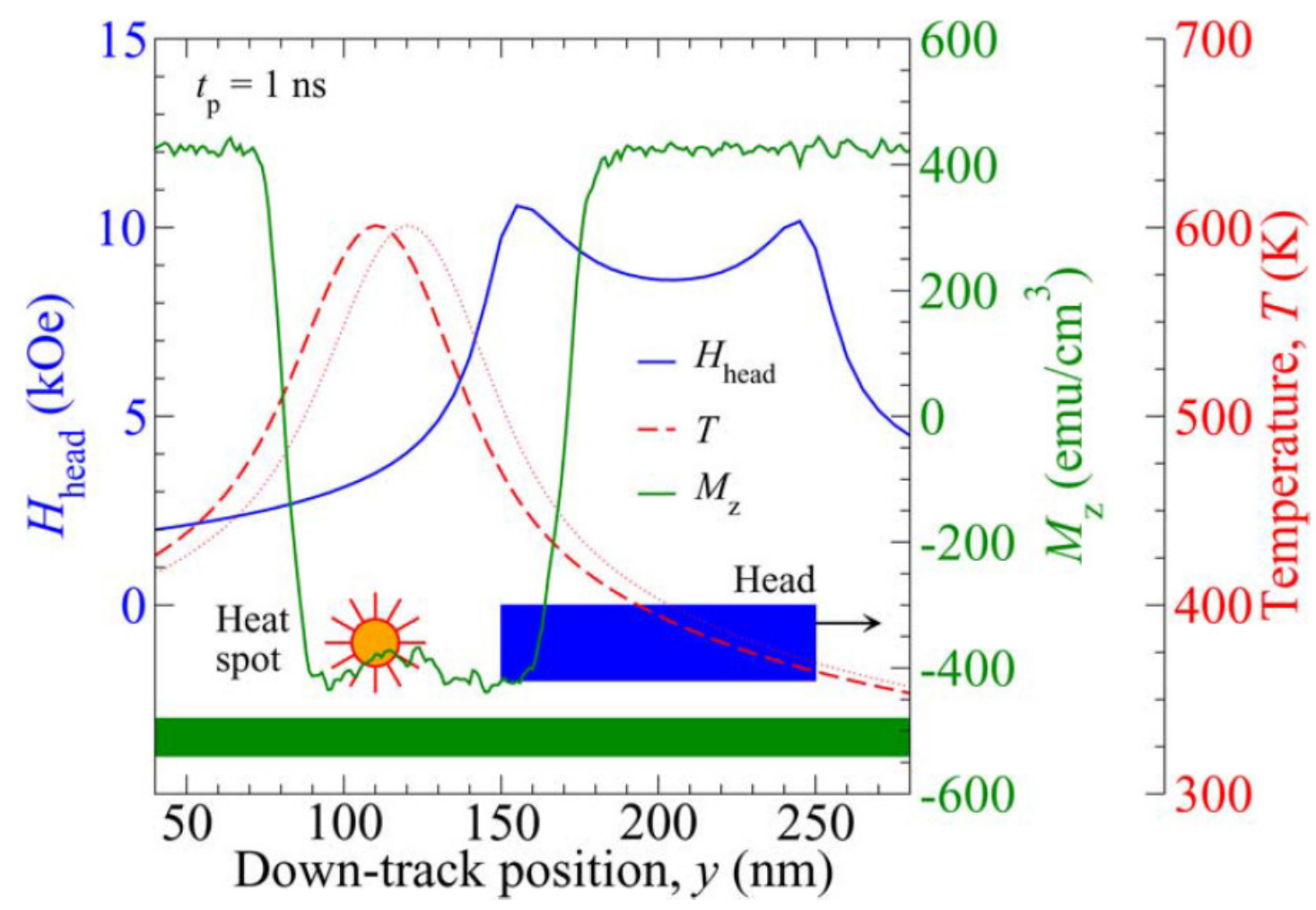

Figure 2.9: Thermal and magnetic field down-track profile during writing process [60].

material anisotropy field $\left(H_{k}(T)\right)$ versus temperature just below the Curie temperature. The second item is the slope of the thermal profile at the freezing temperature. The resulted writing field gradient is 3 to 20 times bigger than the direct field gradient generated by the inductive head [5]. From the above discussion, it is clearly shown that the thermal profile is the dominating factor in the writing process for HAMR drive rather than the writing magnetic field generated from the writer as in PMR drive. The very high writing field gradient in HAMR ensures high linear density in the down-track direction, which is measured in kilo flux change per inch (KFCI). In Figure 2.9, the thermal profile and magnetic field in down-track direction are simulated [60]. In the cross-track direction, the sharp slope of the thermal profile reduces the chance of adjacent track erasure. In addition, the magnetic writer width (MWW) can be varied by adjusting the thermal spot size, which is another major 
difference from PMR drive.

There are still a lot of challenges remaining for HAMR HDDs going into mass production. And the most important two are designing an efficient and reliable light delivery system, especially NFT, and low noise high $K_{u}$ media [58]. In the following sections, more details will be given on these two areas.

\subsubsection{Media for HAMR}

HAMR media is one of the most critical components for the success of HAMR technology. The media contain multiple layers and the basic structure is shown in Figure 2.10. The recording layer contains the magnetic materials for recording the data. Many materials, such as FePt, FePd, CoP, MnAl and $S_{m} C_{o 5}$ [61-66], have been studied. Among these, FePt is so far the best candidate due to its high anisotropy and small grain size of less than $10 \mathrm{~nm}$ in diameter after tremendous research and development [67-74]. Besides the small size of the grain, the distribution of the grain size must be as tight as possible. The variation in the grain size will not only increase the roughness of the media surface, but also increase the variance of the Curie temperature distribution of the media and results in a lower SNR of the recorded signal.

Since the thermal profile is the dominating factor in HAMR recording process, the thermal property of the media is a very important design parameter. In order to have a big gradient for the temperature profile, the heat transmission in the lateral direction of recording layer should be minimized. And good thermal conduction in the axial direction of media stack is desired. To facilitate the heat conduction in the axial direction, a heat sink layer is deployed under the recording layer with the interlayer in between. The heat sink layer must be designed to balance between the temperature gradient and the lifetime of the NFT. It is because more laser power 


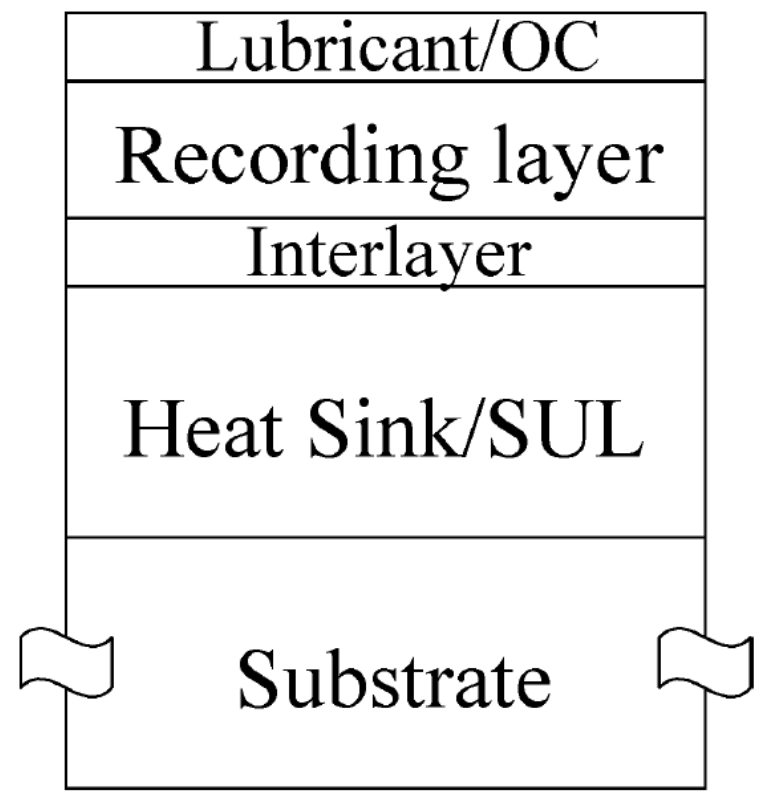

Figure 2.10: Structure of HAMR media [5].

will be needed to heat up the media to the same temperature level of the smaller heat $\operatorname{sink}[75]$.

The lubricant or over-coat layer for HAMR must be redesigned as well to withstand the high temperature and the thermal cycling during the prolonged writing process [5].

\subsubsection{Light Delivery System for HAMR}

The most difficult part in HAMR is to deliver enough thermal power to the media within a very small spot size. When the AD is beyond 1 terabyte $/$ in $^{2}$, the area per bit is around $(25 \mathrm{~nm})^{2}$. Since the media thermal profile is the dominating factor in the recording process of HAMR, the thermal spot size $d$ should be less than $25 \mathrm{~nm}$ as well. However, the traditional far field optics cannot focus the laser light to such a small size due to the diffraction limit. The diffraction limit for the full width at half-maximum (FWHM) optical spot size can be estimated by scalar 


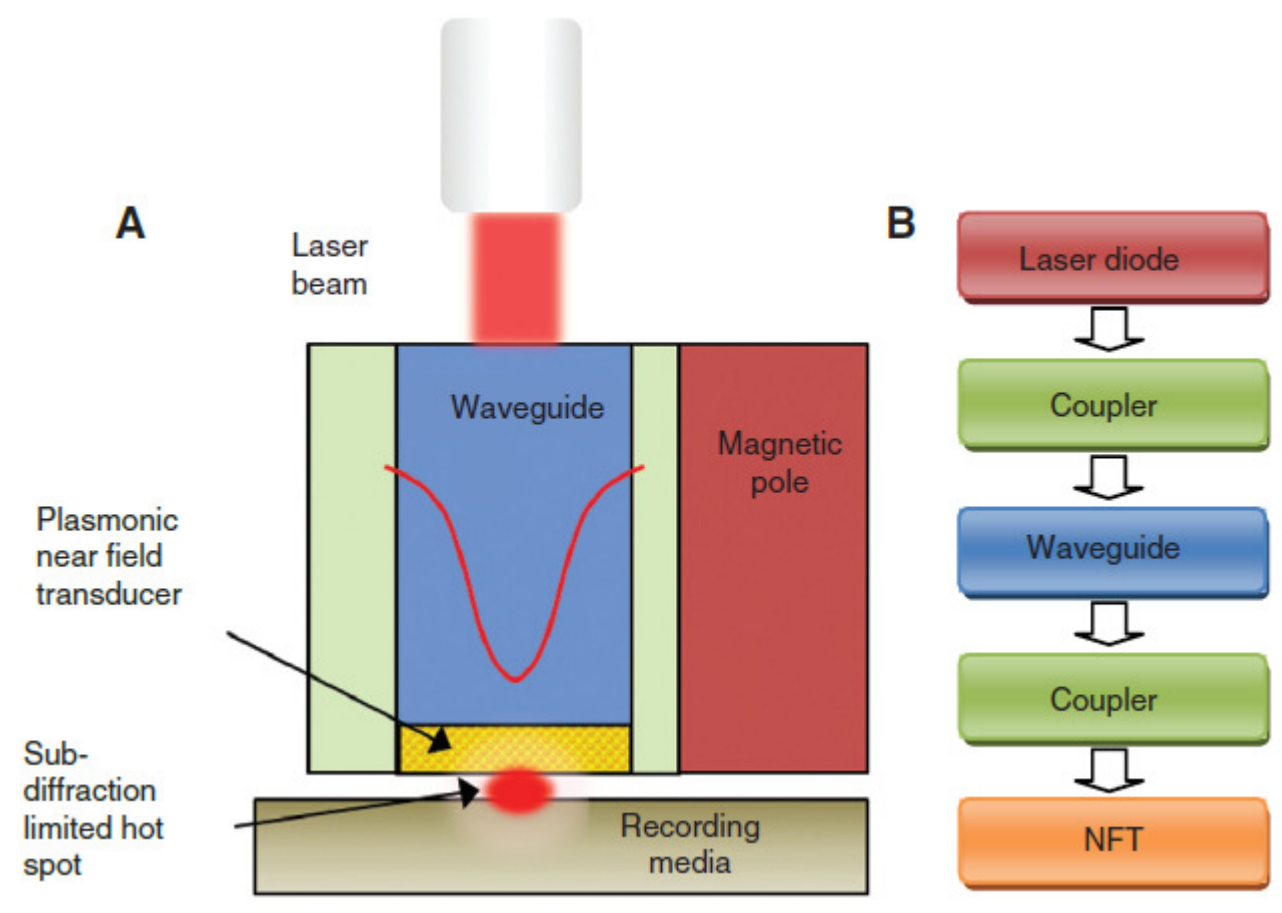

Figure 2.11: Light delivery system of HAMR [76].

diffraction theory as shown in (2.4) where $\lambda$ is the wavelength and $N \cdot A$ is the numerical aperture of the focusing lens [5].

$$
d=\frac{0.51 \lambda}{N \cdot A}
$$

For a good approximation, the minimum optical spot size is roughly the half of the laser wavelength. Since the wavelength of the laser is a few hundred nanometers, the resulting optical spot size is an order bigger than the requirement from HAMR recording even with the light condensers such as the solid immersion lens (SIL) and solid immersion mirror (SIM) [5].

In order to achieve spot size much smaller than the diffraction limit, the nearfield optics, which make use of apertures or antennas, must be used. The overall light delivery system of the HAMR is shown in Figure 2.11 [76]. The laser light is delivered to the waveguide through a coupler. And the waveguide will enhance and focus the laser light to a small spot and then coupled to the NFT. A few waveguides 
have been designed such as the planar solid immersion mirror (PSIM) [77], thin film dielectric waveguide [78] and metallic surface plasmon waveguide [79].

\subsubsection{NFT Design}

The purpose of the NFT is to focus the incident light to a nanometer sized spot, which is much smaller than the laser wavelength by breaking the diffraction limit. This is achieved by the excitation of the surface plasmon of a nano-structure. The strong surface plasmon resonance will enhance the field and radiate it into the media. The much stronger field is only confined in the near field and has a large divergence. However, this is not a concern since the spacing between the NFT and the medium is only a few nanometers during the recording process in HAMR [76].

The other critical design parameters for NFT are efficiency and self-heating. The efficiency should be as high as possible so that less input laser power is needed when delivering required thermal energy to the media. The wasted laser power will be converted to heat dissipated in the head. Besides the conventional heat sources for PMR drive such as heater used for thermal fly-height control (TFC) and writer current, there are more heat sources for HAMR drive, such as laser diode generated heat, scattered light from NFT and even media back heating [80]. All the heat sources for HAMR are illustrated in Figure 2.12.

The NFT itself will also absorb the optical energy and cause the self-heating [77]. The over-heating of NFT is the major failure mode and lifetime bottleneck in the HAMR system. In order to improve the performance of the NFT, many different transducer structures have been studied and developed such as the circular aperture [81-86], C-aperture [87-93], bowtie aperture [94], L-aperture [95], Lollipop NFT $[77,96]$, and beaked plate structure [97].

Currently, gold is widely used as the NFT material because of its high melting 


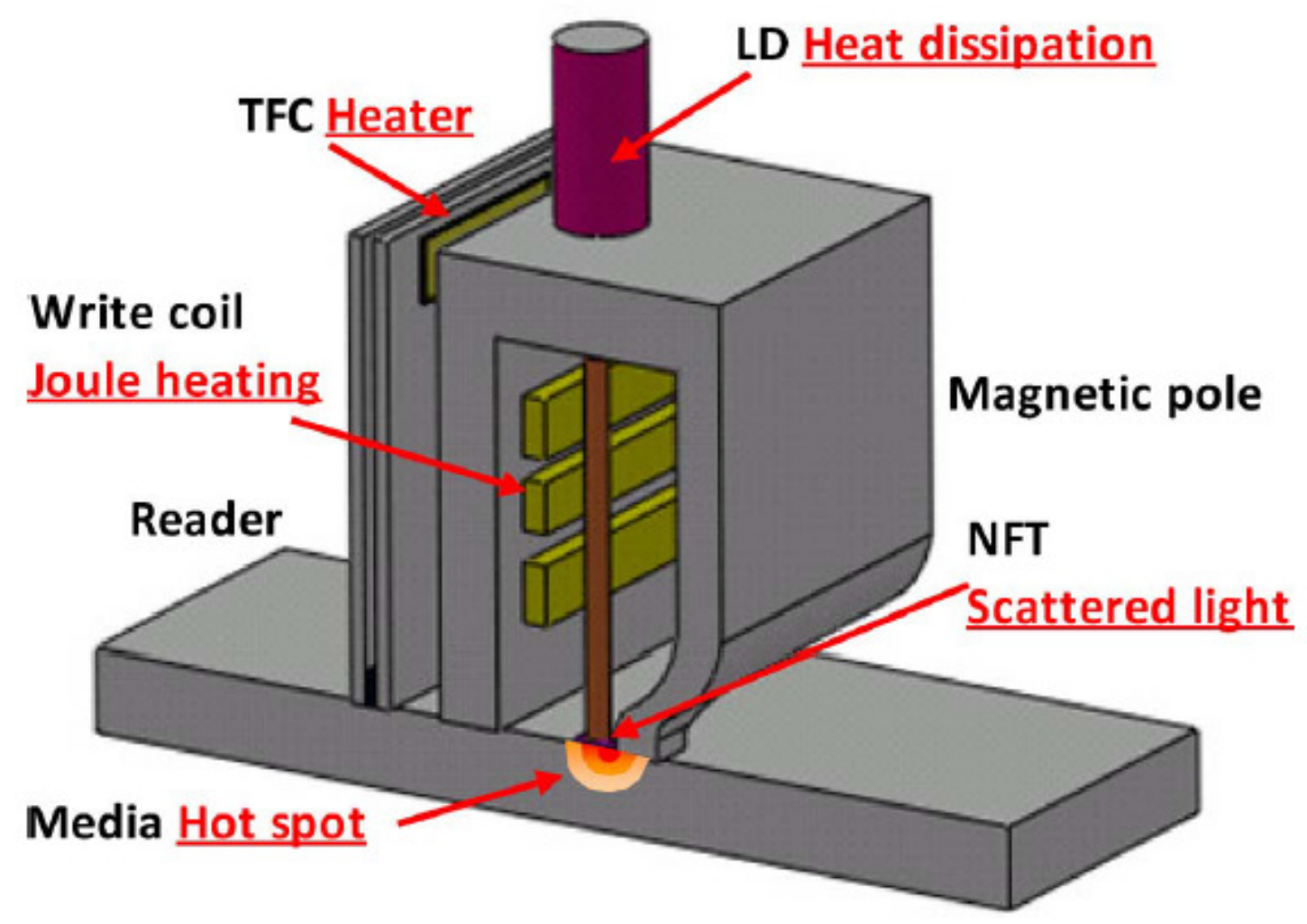

Figure 2.12: Heat sources for HAMR Drive [80].

point, high thermal conductivity, and chemical stability. But in nanometer scale, gold suffers from poor thermal stability at temperatures of much lower than its melting point. Researchers are keeping looking for better alternatives [98] and some alternative plasmonic materials can be loosely categorized as metallic alloys [99,100], semiconductor-based [101], ceramic materials [102], 2D materials such as grapheme $[101,103]$ and organic materials [104].

Another major concern related to the heat dissipation in HAMR is related to protrusions. Multiple heat sources and protrusion components make the NFT flyheight control a very challenging task. The spacing between NFT and media is one of the most important parameters to control during the recording process because it will affect the energy transfer efficiency [105]. Many research works have been done to have a better understanding of various protrusions [106-110]. However, 


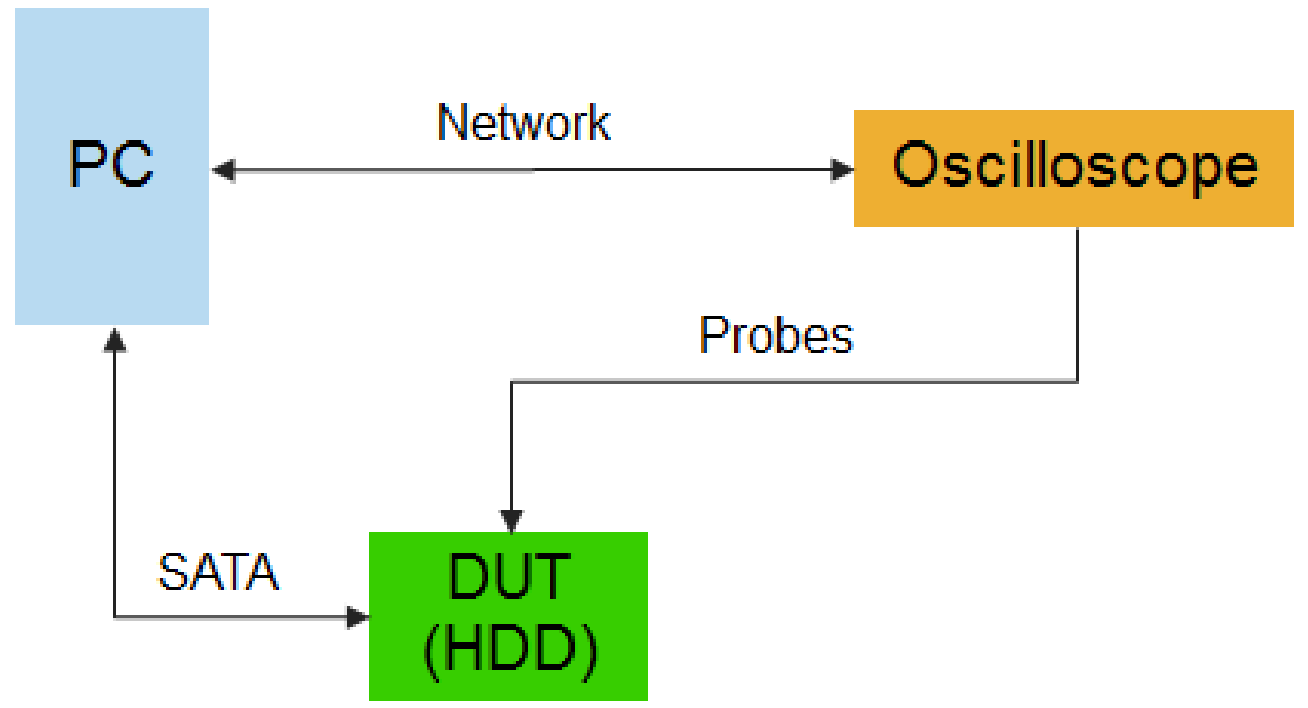

Figure 2.13: Block diagram of the experiment setup.

the accurate direct real-time measurements of protrusions and NMS remain a very difficult task due to the tiny size of the NFT.

\subsection{Experiment Setup}

The block diagram of the experiment setup used for this research work is shown in Figure 2.13. The device under test (DUT) is Western Digital 2.5", 5400 rpm prototype HAMR drive. The DUT is connected to PC through serial advanced technology attachment (SATA) interface. The PC is used to control the operation of the DUT using scripts developed in MATLAB. The desired electrical signals are captured by the oscilloscope through the probes, which are attached to the test pads located on the printed circuit board (PCB) of the DUT. The oscilloscope is connected to a PC through a network so that it can be controlled by the PC. And the captured waveform can be transferred to the PC automatically for processing and analysis. 


\subsection{Summary}

In this chapter, the discussion is focused on the basic concept of HDD servo control system and HAMR technology. HAMR is the most viable technology to bring the AD beyond 1 terabyte $/ \mathrm{in}^{2}$. As discussed, there are a lot of fundamental differences between HAMR and PMR, which make the control of writing process much more complicated for HAMR compared to PMR. As a result, the following two chapters will focus on how to write good quality servo patterns for the HAMR drive with the help of the writing process modeling. Our ultimate goal of this research work is to improve the servo performance of high AD HAMR drives. In the following chapters, a new method will be proposed to minimize the non-linearity of burst pattern and a new RRO compensation scheme called CWRRO is invented to tackle the RRO challenges. 


\section{Chapter 3}

\section{HAMR Writing Modeling and Identification}

\subsection{Introduction}

Current HDDs implement perpendicular magnetic recording (PMR) technology, which contributes to the tremendous AD growth in the past decade. However, it encounters the superparamagnetic limit when the AD approaches 1 terabyte $/ \mathrm{in}^{2}$, where the average bit area is only around $(25 \mathrm{~nm})^{2}[5,59,111-113]$. Heat-assisted magnetic recording (HAMR) technology is expected to bring the AD to beyond a few terabyte $/ \mathrm{in}^{2}$. For HAMR, the recording layer is designed to have high anisotropy at room temperature using materials such as FePt alloys [5]. During the writing process, the media are heated up locally by laser through the near field transducer (NFT) to reduce its coercivity such that the applied writing field is able to magnetize the grains at elevated temperature. The media is then frozen to the desired state when the media temperature is cooled down [5]. For PMR, the magnetic field gradient is the most critical parameter for the head design to achieve good written-in 
signal quality [114]. However for HAMR, the written-in signal quality is determined by both of the media thermal profile and the writing magnetic field, in both of the cross-track direction, which determines the track width, and the down-track direction, which determines the linear density that is measured in kilo flux change per inch (KFCI) $[59,60,96,113]$. In contrast to PMR, the thermal profile dominates in HAMR writing process. The main challenges for HAMR technology are efficient and reliable NFT design, and low noise high thermal gradient media design [58,115-117]. Efficient NFT can deliver more thermal energy to the media while reducing the selfheating and extend its own lifetime. People are designing different types of NFT to increase its efficiency and lifetime [76,118-120]. In addition, tremendous efforts are spent on media design to improve the SNR and AD [56,60,75,111,119,121-134]. Besides the head and media design, another major factor affection the thermal profile is the NFT tip to media spacing (NMS) $[105,135,136]$. NMS keeps changing during the writing process due to the laser heat dissipation generated protrusions, on top of the usual head-to-media spacing (HMS) change due to thermal fly-height control (TFC) [137-142] and write current [121]. The HAMR related heat sources consist of laser diode generated heat, laser light converted heat, and back heat from the media $[80,112,121,143]$. In order to understand the NMS, Rausch et al. [109] use automatic gain control (AGC) to track the NMS changes during the writing process. Atomic force microscopy (AFM) is used to image the NFT protrusion on the air bearing surface (ABS) with a nano-scale resolution [106]. The thermal protrusion and temperature rise responses versus time as a function of input laser power and NMS are studied in [144]. Burnish method is used to report laser induced protrusion [107]. Nevertheless, as pointed out by Schreck et al. [108], comparing thermal-mechanical modeling and experiments during flying and static conditions remains a critical issue that needs further improvements.

On the other hand, the disk drive servo community routinely measures the actuator's input-output response and extracts the actuator model parameters for servo 
control design and optimization purpose to support the low-cost mass-produced hard disk drives at over 500,000 tracks per inch today [30,145-147]. Uwe et al. [137] report a circuit model for the resistance heater element in a TFC slider and identified the model parameters by matching the system's inputs and outputs data for dynamic flying height control, following a similar system modeling and identification process that is widely performed in control and robotics communities [148-151].

The objective of this chapter is to construct and identify a dynamic model for HAMR writing process. As mentioned in [149], there are generally two methods to construct a model. One is to build models to describe the relationship between input and output without considering any physical insight of the system. The other method is to build the model based on the underlying physical phenomenon of the system. The system identification methods can be classified into two categories, nonparametric identification methods and parameter estimation [149]. Non-parametric methods includes transient analysis, correlation analysis and spectral analysis and so on. The parameter estimation method tries to fit the parameters to the obtained data by minimizing the prediction error. It is very straightforward if the model is linear. If the model is very complex or non-linear, iterative search for the minimum prediction error is needed [149].

In this chapter, the HAMR writing process is modeled with laser power and TFC heater power as inputs, and signal magnitude as the output. First, the energy transfer efficiency in HAMR writing process is identified which determines how much laser power can be transformed into heat energy in the recording layer. Next, the model parameters describing the NFT protrusion with respect to the laser current will be established. The NFT protrusion will change NMS and then the energy transfer efficiency as a result. After that, the heat transient process which is in nanoseconds range will be characterized to make a good estimation for the first few written-in data bits. Finally, the relationship between the thermal profile and 
written-in signal magnitude is modeled and identified. Since the the thermal profile is not measurable in drive, it can be estimated by the measured signal magnitude through their relationship. By measuring the written-in signal magnitude response at a given writer's head flying height, prelase laser power as well as active laser power, the Gaussian-Newton method is then used to identify all the parameters of this non-linear system model. As a result, our problem is categorized as a nonlinear model parameter estimation problem, where the Gaussian-Newton method is the iterative method applied. The Gaussian-Newton method does not guarantee the convergence of the result. As a result, the identification process is carried out in such a way that the convergence is verified to have been achieved. The identified parameters are able to predict the written-in signal amplitude reasonably good under different configurations.

As one of the applications of the writing process model, it is used to select the active laser power and TFC adjustments for writing a very short pattern (relative to the regular user data) based on the user data writing conditions, which were derived from optimization and calibration processes. The model clearly shows the allowable TFC adjustments to compensate for the NMS change due to the reduced NFT protrusion arising from the reduced written data length, and allows us to write data with the same quality regardless of the length of the data to be written.

On the other hand, the model can be used to find the optimized laser overshoot current profile to compensate for the transient response of the writing process to generate an output as close as possible to a step function. The overshoot current has an exponential decay profile and is characterized by the overshoot amplitude (OSA) and overshoot duration (OSD). OSA determines the initial laser current value and OSD is the time constant of the exponential decay. 


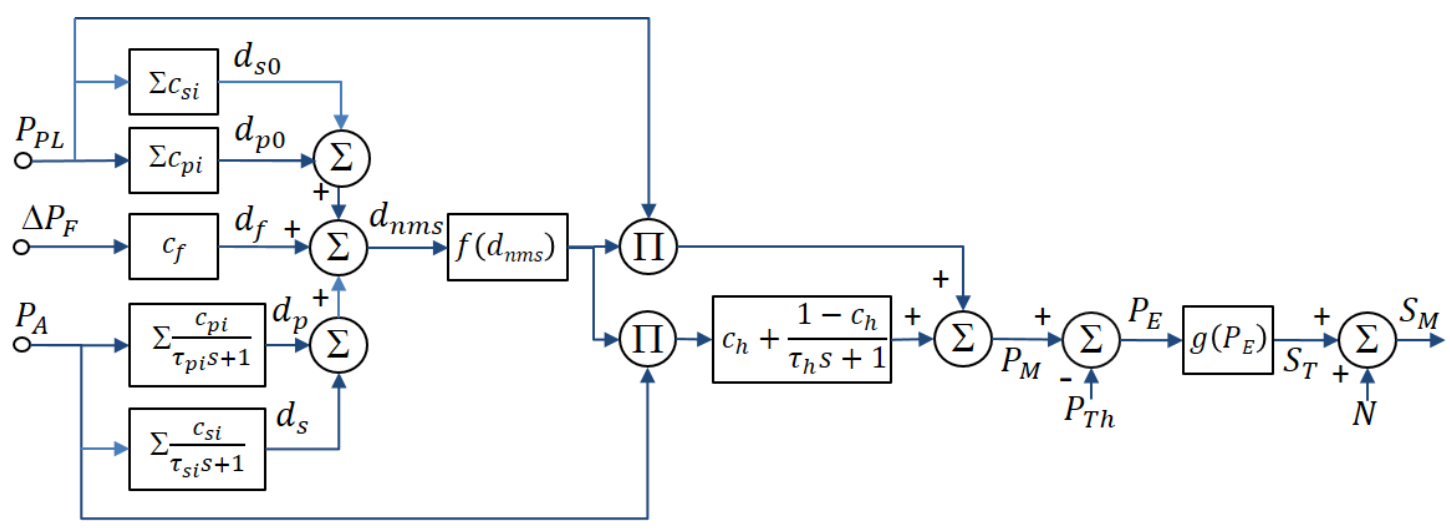

Figure 3.1: Dynamic model for HAMR writing process.

\subsection{HAMR Writing Model Identification}

The HAMR writing process is treated as a system with laser power and TFC heater power as the input and signal magnitude as the output. In HAMR, the signal magnitude is directly related to the input laser power and the NMS. In addition, the laser power generated heat will cause various protrusions and further reduce the NMS. The completed model is shown in Figure 3.1. The model parameters will be identified in a way such that the predicted read-back signal $S_{M}$ can match with those measured from the real drives.

The TFC heater power $\Delta P_{F}$ is used to control the static flying height $d_{f}$ before the actual writing process starts. It is applied early enough so that the $d_{f}$ can be considered a constant during the whole writing process. In our model, there are two kinds of protrusions related to laser power, one has a fast time response related to NFT protrusion itself and the another one corresponds to the slider protrusion which is slower than the fast one by the order of two. Since these two protrusions have very different time responses, they can be identified separately. In the following section, the focus is on the explanation of the model structure and the identification process of the fast protrusion parameters, and the slow one is assumed in the steady state already. The slow protrusion parameter identification will be discussed in the 
Table 3.1: List of Symbols

\begin{tabular}{|c|c|}
\hline \multicolumn{2}{|r|}{ Input } \\
\hline$\Delta P_{F}$ & Heater power for TFC control, in $m W$ \\
\hline$P_{A}$ & Active laser power, only applied during write, in $m W$ \\
\hline$P_{P L}$ & $\begin{array}{l}\text { Prelase laser power, applied before actual write for pre-heat, and dur- } \\
\text { ing write, in } m W\end{array}$ \\
\hline \multicolumn{2}{|r|}{ Output } \\
\hline$S_{M}$ & Measured signal magnitude, in $m V$ \\
\hline$S_{T}$ & True signal magnitude, in $m V$ \\
\hline$N$ & Noise floor after AC erase, in $m V$ \\
\hline \multicolumn{2}{|r|}{ Variables not measurable } \\
\hline$d_{p}, d_{p 0}$ & NFT fast protrusion due to $P_{A}$ and $P_{P L}$, in $n m$ \\
\hline$d_{s}, d_{s 0}$ & Slow protrusion due to $P_{A}$ and $P_{P L}$, in $n m$ \\
\hline$d_{f}$ & $\begin{array}{l}\text { HMS change due to non-laser related factors such as TFC and write } \\
\text { current etc, in } n m\end{array}$ \\
\hline$d_{n m s}$ & Total NMS change, in $\mathrm{nm}$ \\
\hline$P_{M}$ & Normalized thermal power on media \\
\hline$P_{E}$ & Normalized effective thermal power \\
\hline \multicolumn{2}{|r|}{ Unknown parameters to be identified } \\
\hline$P_{T h}$ & Normalized threshold thermal power \\
\hline$c_{p i}$ & Gain for NFT fast protrusion, $i=1,2$ if subscripts used \\
\hline$\tau_{p i}$ & $\begin{array}{l}\text { Time constants for NFT fast protrusion, } i=1,2 \text { if subscripts used, in } \\
\mu s\end{array}$ \\
\hline$c_{s i}$ & Gain for slow protrusion, $i=1,2$ if subscripts used \\
\hline$\tau_{s i}$ & Time constants for slow protrusion, $i=1,2$ if subscripts used, in $\mu s$ \\
\hline$c_{h}$ & Gain for heat transient process \\
\hline$\tau_{h}$ & Time constants for heat transient process, in $n s$ \\
\hline \multicolumn{2}{|r|}{ Known parameters } \\
\hline$c_{f}$ & Gain for TFC, in $n m / m W$ \\
\hline
\end{tabular}


later portion of this chapter.

\subsubsection{Structure of HAMR Writing Model}

The model mainly consists of four parts, the NFT protrusion model, the energy transfer efficiency function $f\left(d_{n m s}\right)$, the heat transient process, and the power-tosignal conversion function $g\left(P_{E}\right)$. This model is called a dynamic model because it describes the input-output relationship as a function of time. The descriptions of all the symbols used in the model are listed in Table 3.1. Our system identification can be considered as the "Grey-Box" identification. In this section, it will be explained on how the model is constructed based on existing knowledge of the HAMR technology.

\subsubsection{NFT Protrusion}

The major heat source for the temperature increase of NFT is the self-heating during laser producing and delivering processes. The another heat source is the back-heating from the media since NFT is closest to the media. Temperature rise due to back-heating is small and can be ignored [80]. It has been shown by the simulation that the temperature rise of NFT is linearly dependent on the laser power [121]. Some methods are also used to measure the NFT temperature $[80,119,143]$. The temperature rise in NFT will cause it to protrude and reduce the NMS. NFT protrusion is found to have an approximately linear relationship with laser input current for a non-flying head using AFM-based method [106]. As a result, it is assumed that the steadystate NFT protrusion is proportional to the effective laser power (laser current used to turn on the laser diode is not included in the laser power calculation since there is no laser output). The dynamic response of NFT protrusion is modeled as a second order system with time constants $\tau_{p 1}$ and $\tau_{p 2}$ which are to be 


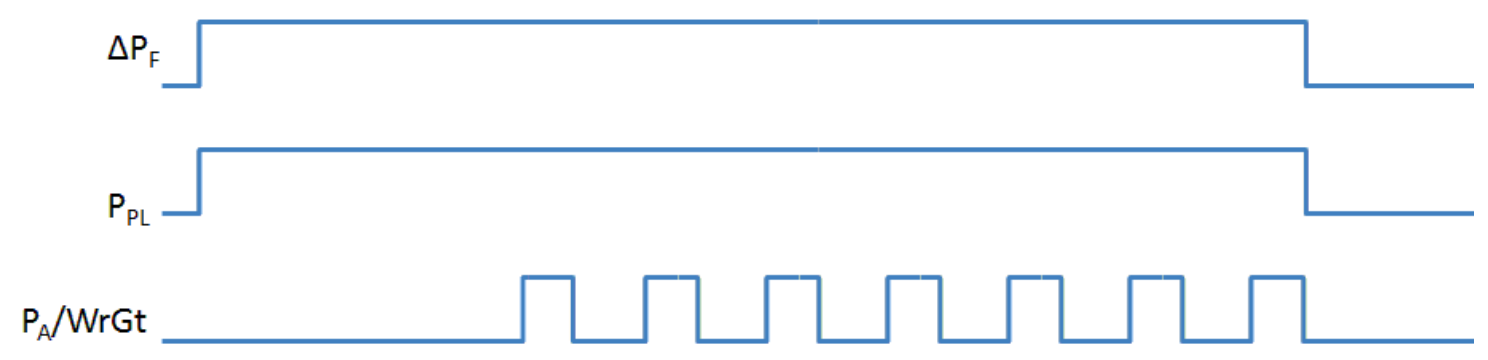

Figure 3.2: Input power sequence.

identified. The steady state protrusion is proportional to input laser power and the gains to be identified are denoted as $c_{p 1}$ and $c_{p 2}$. Owning to the localized heating of the NFT only, the time constant of this mode is expected to be around $1 \mu s$ through thermal-mechanical simulation.

In our HAMR writing process, a small laser power, the so-called prelase $P_{P L}$, is applied some time before the actual write starts for the pre-heat purpose. The media temperature rise due to prelase should be safely low enough so as not to erase the pre-written servo patterns. The NFT protrusion due to this power reaches its steady state before actual writing starts and is denoted as $d_{p 0} . P_{A}$ is called active laser power which is applied during actual write and is synchronized with the write gate. The NFT protrusion caused by $P_{A}$ is denoted as $d_{p}$. The input power time sequence is illustrated in Figure 3.2. Please note that for simplicity of the identification process, $\tau_{p 1}$ and $\tau_{p 2}$ are lumped as $\tau_{p}, c_{p 1}$ and $c_{p 2}$ are lumped as $c_{p}$. It is found that such lower order approximation gives good matching between the modeled and measured signal amplitudes.

\subsubsection{Power-to-Signal Conversion}

Noise mechanics in HAMR have been extensively studied [128, 152-154]. In this section, only the effect of media thermal profile is considered by assuming that the write magnetic field is already optimized. In terms of the media properties, 
only the distribution of grain Curie temperature is considered, and the effect of anisotropy field is ignored since its effect is largely suppressed by the high effective field gradient [152]. The media thermal profile is determined by the energy delivered to the particular media. Media temperature and power are not measurable in the real drive, hence they are not available for writing model parameter identification. Fortunately, there is a strong link between the written-in signal quality and the thermal profile, since the thermal profile determines whether the media grains are raised to a suitable temperature, i.e., above the curie temperature for most, if not all of the magnetic grains. Hence, the high thermal gradient ensures high effective field gradient for high-density recording. Assuming that the read process is normalized and the read-back signal is determined by the media magnetization but not reader, a single tone written-in signal's magnitude $S_{M}$ is measured by taking the moving average of the absolute value of the read-back signal. The moving average window is set the same as the period of the signal. The magnitude of noise floor after AC erase is denoted as $N . S_{T}$ is the true signal magnitude derived by subtracting $N$ from $S_{M}$.

Media thermal profile is determined by the media design, which is discussed extensively $[121,129,132]$. A 2-D Gaussian distribution in xy-plane is used to model the thermal profile by assuming uniformity in the z-direction as proposed in [113], where $\mathrm{x}$ is in the down-track direction and $\mathrm{y}$ is in the cross-track direction. Since the $\mathrm{x}$-direction thermal profile and head velocity determine the maximum written-in signal frequency, its effect on signal quality can be ignored by limiting the written-in signal frequency well below the switching limit. In this case, it is only necessary to consider the thermal profile in the y-direction or cross-track direction and its effect on signal magnitude. The temperature at the heat spot center is highest and denoted as $T_{\max }=T(y=0)$.

The Curie temperature for each magnetic grain is not a constant due to process 


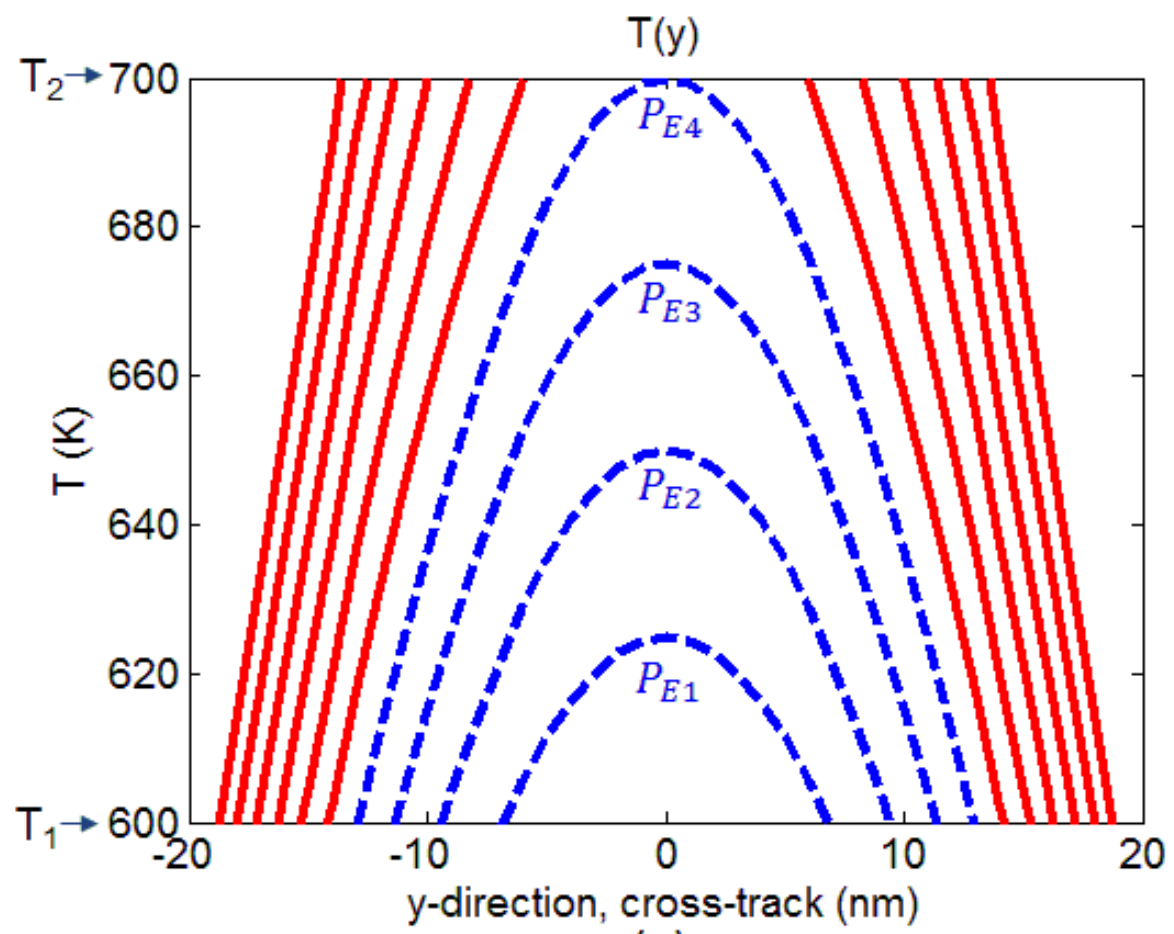

(a)

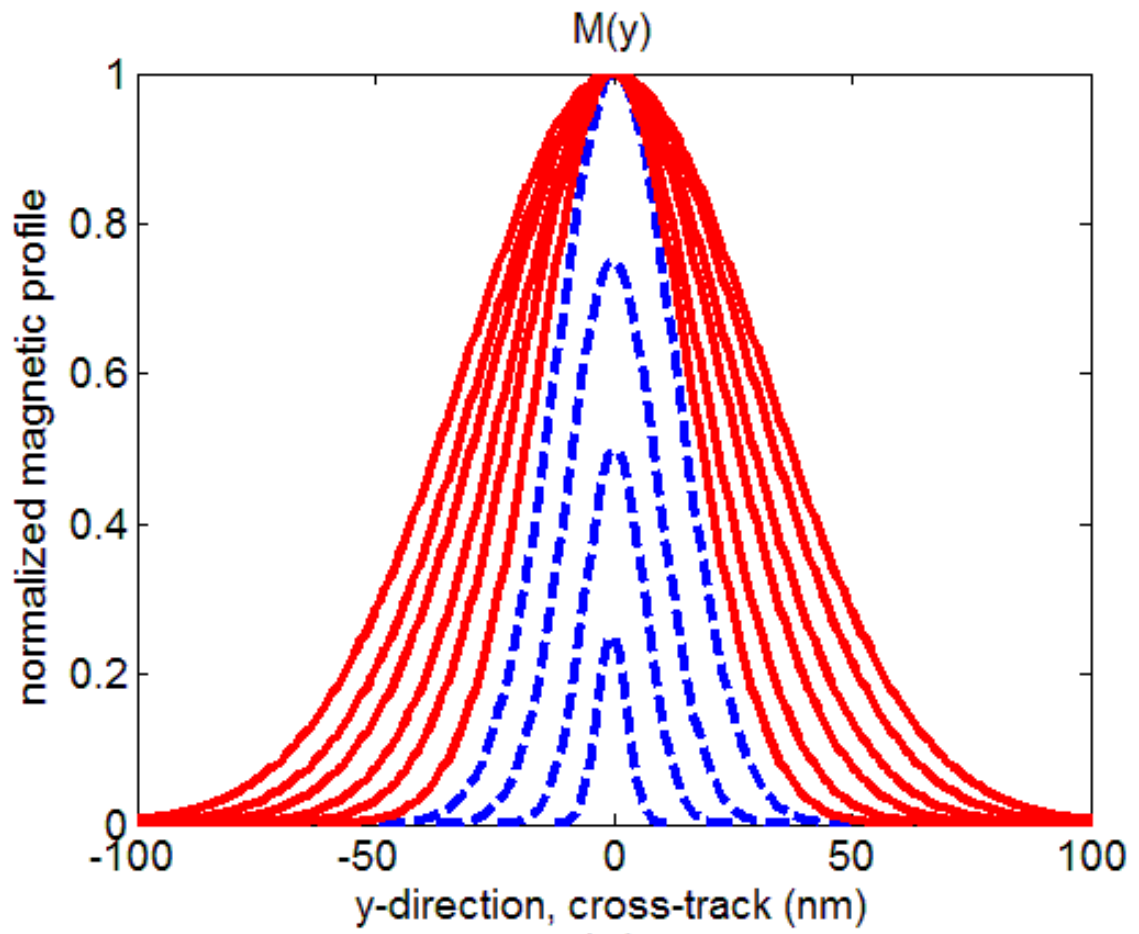

(b)

Figure 3.3: (a) 1-D thermal profiles with different effective thermal power - $T(y)$, $T_{1}=600 K, T_{2}=700 K$; (b) Corresponding 1-D magnetic pattern $M(y)$. 
and material tolerances such as grain size, shapes, composition, chemical ordering and strain and so on $[130,131,155]$. The modeling and measurement of Curie temperature distribution have been discussed $[130,131]$ and people are trying to simulate the magnetic switching performance near the Curie temperature of non-ideal media $[125,127,128,133]$. In our model, the Curie temperature is approximated by a Gaussian distribution. Temperature $T_{1}$ and $T_{2}$ can be considered as the temperature of \pm 3 standard deviation away from the mean Curie temperature of the distribution. The writable temperature range is denoted as $\left[T_{1}, T_{2}\right]$. When the media temperature is below $T_{1}$, there is no grain can be switched. If the media temperature is above $T_{2}$, all the grain are considered switchable. The effective thermal power $P_{E}$ is defined as the power to raise the heat spot temperature $T_{\max }$ from $T_{1}$ upwards. It is assumed that $P_{E}(T)$ is proportional to $T$, where $T=T_{\max }-T_{1}$, and $P_{E}(0)=0$. In this thesis, a 1-D Gaussian distribution is used to model the y-direction written-in magnetic pattern $M(y)$ on the media as shown in (3.1) for the different thermal profile. When $T_{\max }<T_{1}$, there is no switchable grain and $M(y)=0$ everywhere. When $T_{\max } \in\left[T_{1}, T_{2}\right]$, the area whose temperature is above $T_{1}$ is a circle whose radius increase with the $T_{\max }$. Except near the boundary of the temperature range, it is assumed that the number of switching grain increases linearly with the temperature so that $M_{\max }$ increases with $T_{\max }$ linearly. Since the area of the circle is small, the standard deviation of $M y$ is set at constant in this stage. The circle becomes an annulus when $T_{\max }>T_{2}$ and its size increases with $T_{\max }$. Since all the grains are switchable when the temperature is above $T_{2}$, the height of the magnetic pattern $M(y)$ will not increase any more. Instead, its top becomes wider and wider following the annulus size. The magnetic pattern widening effect is simulated by increasing the standard deviation of $M(y)$. The different temperature profiles and 
corresponding magnetic patterns are shown in Figure 3.3.

$$
\left\{\begin{array}{l}
M(y)=0, T_{\max }<T_{1}, \\
M(y)=\frac{P_{E}\left(T_{\max }-T_{1}\right)}{P_{E}\left(T_{2}-T_{1}\right)} \exp \left(-\frac{y^{2}}{2 \sigma_{0}^{2}}\right), T_{\max } \in\left[T_{1}, T_{2}\right] \\
M(y)=\exp \left(-\frac{y^{2}}{2 \sigma^{2}}\right), \sigma=\frac{P_{E}\left(T_{\max }-T_{1}\right)}{P_{E}\left(T_{2}-T_{1}\right)} \sigma_{0}, T_{\max }>T_{2} .
\end{array}\right.
$$

By convolution of the magnetic patterns with the reader micro-track profile, whose shape is also modeled as a Gaussian distribution, the relationship between the signal magnitude $S_{T}$ and the effective thermal power $P_{E}$ can be derived as shown in Figure 3.4. When $T_{1}<T_{\max }<T_{2}$, the magnetic strength at the hot spot center $M(y=0)$ increases linearly with the temperature $T_{\max }$. Since the standard deviation of $M(y)$ profile is kept constant, the area under $M(y)$ has an approximately quadratic relationship with $T_{\max }$. Furthermore, because the width of the magnetic pattern is much smaller than the reader width in this case, the powerto-signal function can be approximated by a square function after convolution, i.e., $S_{T}=g\left(P_{E}\right) \approx P_{E}^{2}$.

When $T_{\max } \geqslant T_{2}$, the height of magnetic pattern $M(y)$ will not increase anymore. Instead, its width will increase with $T_{\max }$. When its width is comparable to the reader micro-track profile, the slope of signal magnitude versus effective thermal power will decrease. The slope will approach zero when magnetic pattern width is much bigger than the reader width. The drive measurement data and the simulation results are compared in Figure 3.4 and they match with each other quite well. In the same figure, the two different trends are clearly shown. In the left portion of the figure, the trend is governed roughly by a quadratic relationship, and the points marked with $P_{E 1}$ to $P_{E 4}$ are corresponding to the thermal profiles and magnetic patterns of the same marking shown in Figure 3.3. In the right portion of the figure, the read-back signal magnitude becomes less sensitive to the increase of media ther- 


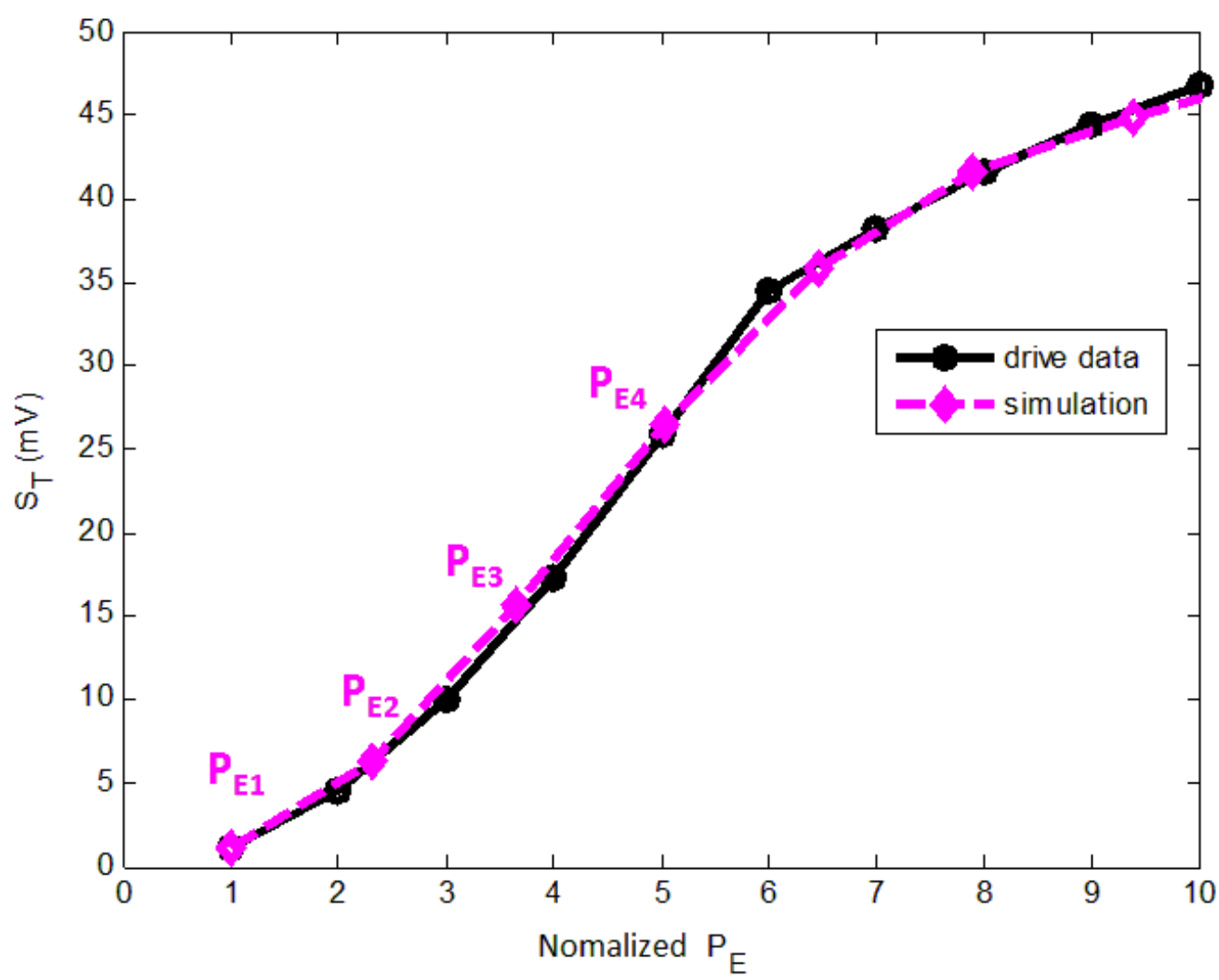

Figure 3.4: True signal magnitude $S_{T}$ vs. normalized effective thermal power $P_{E}$.

mal power. In this thesis, the left portion is called the linear region since $M(y=0)$ increases linearly with $T_{\max }$, and the right portion is called the non-linear region.

\subsubsection{Heat Transient Process}

The thermal power delivering process to the media hotspot is very complex. It is assumed that the steady state temperature at the hot spot center is proportional to the effective input laser power. The temperature rise at the hot spot center after a step change in laser power is shown in Figure 3.5. The initial temperature rises to $T_{i}$ is very fast and may be completed in $1 \mathrm{~ns}$ [119] and is approximated by a step change. However, there is a slower transient in $100 \mathrm{~ns}$ range observed. It is possibly due to other heat sources and the moving head [156]. It is assumed that temperature rises from $T_{i}$ to the steady state $T_{A}$ exponentially with the time constant of $\tau_{h}$. It 


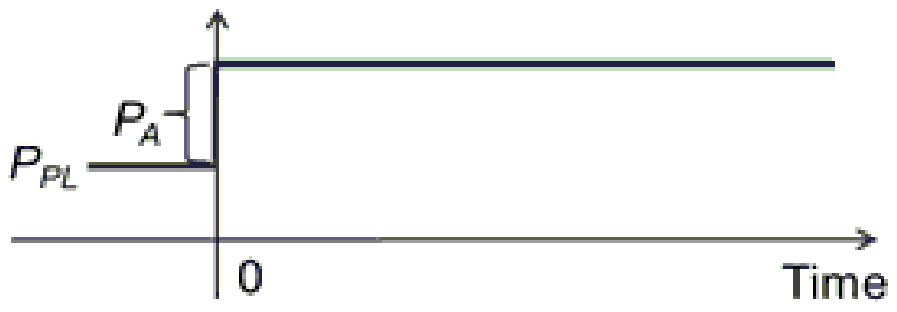

(a)

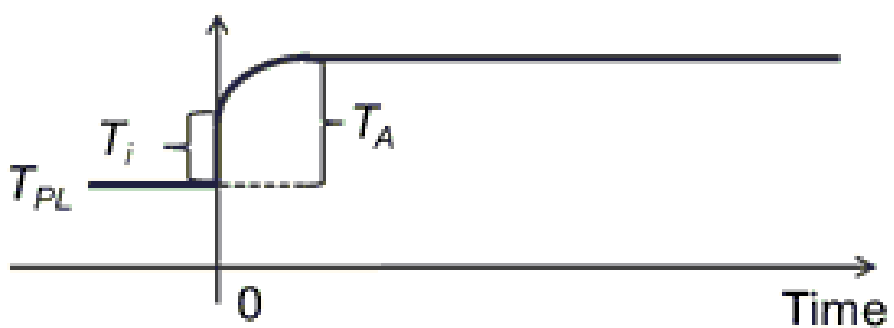

(b)

Figure 3.5: Heat transient process illustration.

is further assumed that the ratio $c_{h}=\frac{T_{i}}{T_{A}}$ is a constant. The whole heat transient process can then be represented by (3.2).

$$
c_{h}+\frac{1-c_{h}}{\tau s+1}
$$

\subsubsection{Energy Transfer Efficiency}

Energy transfer efficiency determines how much laser power can be transferred to the media so as to heat up the magnetic grains. Given a particular head and media, the efficiency is mainly determined by the space between the tip of the NFT and the media. The relationship between the efficiency and NMS can be approximated by an exponential decay function [105]. In this thesis, this relationship is approximated as a second order polynomial equation as shown in (3.3).

$$
f\left(d_{n m s}\right)=c_{2} d_{n m s}^{2}+c_{1} d_{n m s}+c_{0} .
$$


Smaller $c_{1}$ and $c_{2}$ indicate that the write process is less sensitive to the NMS change. Coefficient $c_{0}$ represents how efficient the head and media combination has been given the default fly-height.

\subsubsection{Experiment Setup}

All our data are collected from Western Digital 2.5" 5400 rpm HAMR drive. Above section discussed the building blocks of the writing process model. Since each block represents a different physical phenomenon, their parameters are independent to each other. However, it is very difficult to identify all the parameters at once since the system is a non-linear system and the results may not converge at all. Fortunately, since the slow and fast protrusion and heat transient process all have very different time constants, it is possible to identify the parameters in groups. The setup described in this section is mainly used for fast NFT protrusion identification. Experiment setup specific to slow protrusion identification will be discussed in the later section of this chapter. During data writing process, prelase laser current $I_{P L}$ is fixed at all times, but active laser current $I_{A}$ and fly-height are swept in the pre-defined ranges. The active laser current can be a step function with or without overshoot. For model identification, only step inputs are used. The written-in signal is a single tone signal of $75 \mathrm{MHz}$. The length of the signal is set at $4 \mu \mathrm{s}$ so that the NFT protrusion already reaches its steady state at the end of writing. During data collection, a digital oscilloscope is connected to the hard drive, and Matlab is used to read the raw waveform directly from the digital scope. Then the raw signal is converted to signal magnitude by calculating the moving average of the absolute value of the read-back signal. The moving window size is set as the period of the written-in single tone signal. The input laser power together with the raw waveform and calculated signal magnitude is illustrated in Figure 3.6. The signal magnitude calculated from the last 50 wedges are utilized, where the slow protrusion has already 

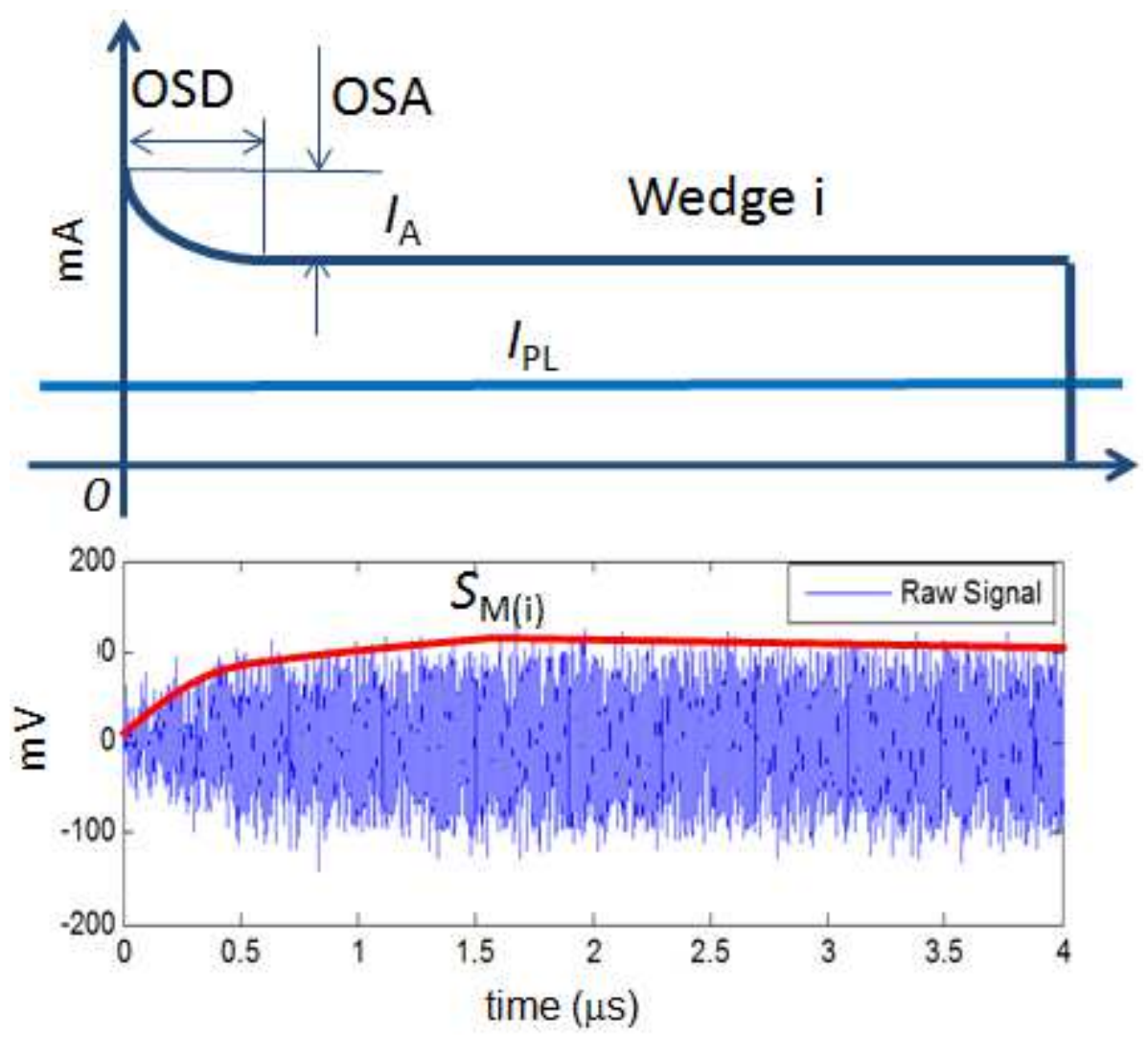

Figure 3.6: Experiment setup.

settled down. Since the fast NFT protrusion repeats itself for every wedge, all the 50 wedge's data are averaged to remove the measurement noise.

\subsubsection{System Identification}

In this section, details will be discussed on how to identify all the parameters given the data collected from the setup mentioned in the previous section. First of all, Let's summarize all the assumptions made in the above section:

- Temperature rise of NFT is linearly dependent on the laser input power [121].

- NFT protrusion amount is linearly dependent on the laser input power [106].

- Steady state temperature at the hot-spot center is proportional to the effective 
input laser power.

- The dynamic of NFT protrusion is modeled as a first order system with time constant $\tau_{p}$ and gain $c_{p}$.

- Only the distribution of the magnetic grain Curie temperature is considered in the model, the effect of anisotropy field is ignored due to its weak effect [152]. The magnetic grain Curie temperature is approximated by a Gaussian distribution.

- A 2-D Gaussian distribution in xy-plane is used to model the media thermal profile by assuming uniformity in the z-direction [113].

- The written-in magnetic pattern $\mathrm{M}(\mathrm{y})$ is modeled as a Gaussian distribution.

- Effective thermal power $P_{E}(T)$ is assumed to be proportional to $T$.

- The temperature rising process of the media can be modeled by (3.2).

- The relationship between energy transfer efficiency and NMS can be approximated by a second order polynomial equation (3.3).

By examining the read-back signal magnitude shown in Figure 3.7, two dynamics in the process can be found. One is in 100 ns range, which corresponds to the heat transient process. The other is in $1 \mu s$ range, which is caused by the NFT fast protrusion. Since the two time constants have been separated by roughly one order in magnitude, they can be identified separately by using the signal magnitude at the different time frame. 


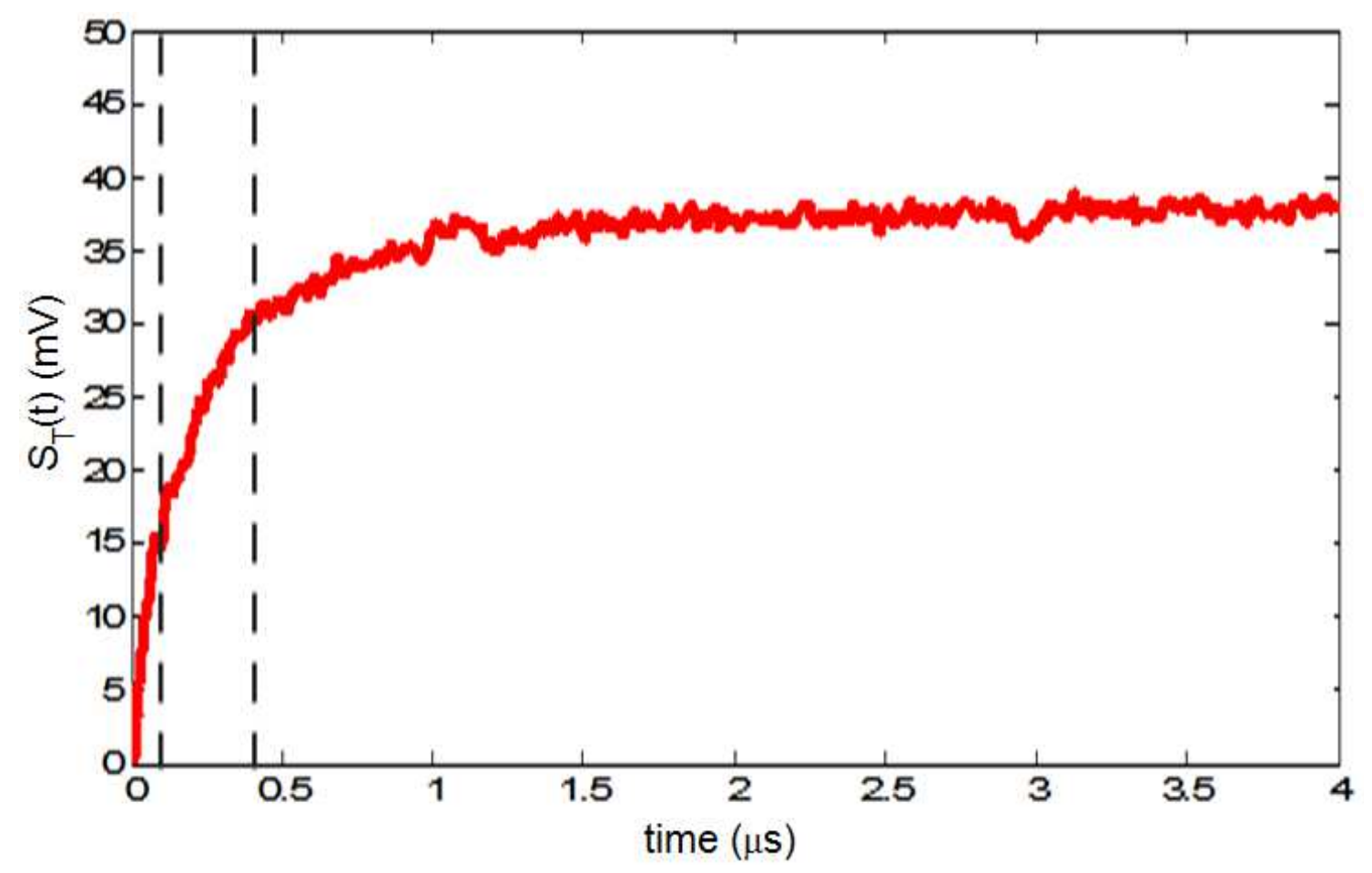

Figure 3.7: One example of read-back signal magnitude.

\subsubsection{Identify $f(x)$, NFT Protrusion Gain and Time Constant, and Threshold Thermal Power}

In this section, the gain and time constant for NFT fast protrusion together with energy transfer efficiency function $f\left(d_{n m s}\right)$ and threshold thermal power $P_{T h}$ will be identified first. The signal magnitude from $0.4 \mu s$ onward is used to identify the parameters since the heat transient process has almost completed in this period. The increase of signal magnitude in this period is only due to the NFT protrusion, which reduces the $d_{n m s}$ and increases the energy transfer efficiency.

Without considering the heat transient process, the writing process is then governed by (3.4) when the active laser power $P_{A}$ is a step input.

$$
\begin{aligned}
f\left(d_{f}+c_{p}\left(P_{P L}+\left(1-e^{-t / \tau_{p}}\right) P_{A}\right)\right)\left(P_{P L}+P_{A}\right)-P_{T h} & =P_{E}(t) \\
& =S_{T}(t)^{1 / 2} .
\end{aligned}
$$


As shown, this model is highly non-linear and the Gaussian-Newton method is going to be used to identify the parameters. It is found that the identification process will not converge if all the parameters mentioned above are to be identified at once. As a result, the time constant $\tau_{p}$ is fixed first based on the observation and the other parameters are then identified. Next, the $\tau_{p}$ is swept until the minimum estimation error is obtained. In this way, the identification always converges.

First, an initial guess is given for $\tau_{p}=1 \mu s$ based on the waveform shown in Figure 3.7. The gain for TFC $c_{f}$ is known, and the fly-height change due to TFC can be calculated as $d_{f}=c_{f} * \Delta P_{F}$. Then, the unknown vector is defined as $w=\left[c_{2}, c_{1}, c_{0}, c_{p}, P_{T h}\right]^{\prime}$. Finally, the residue function is defined as $r(w)$ as shown in $(3.5)$.

$$
r(w)=f\left(d_{f}+c_{p}\left(P_{P L}+\left(1-e^{-t / \tau_{p}}\right) P_{A}\right)\right)\left(P_{P L}+P_{A}\right)-P_{T h}-S_{T}(t)^{1 / 2} .
$$

It is now formulated as a non-linear least squares problem to minimize $\|r(w)\|^{2}$ which can be solved using the Gaussian-Newton method.

Linearize $r(w)$ near current iteration $w^{(k)}$ :

$$
r(w) \approx r\left(w^{(k)}\right)+\operatorname{Dr}\left(w^{(k)}\right)\left(w-w^{(k)}\right)=A^{(k)}-b^{(k)},
$$

where $A^{(k)}=\operatorname{Dr}\left(w^{(k)}\right), b^{(k)}=\operatorname{Dr}\left(w^{(k)}\right) w^{(k)}-r\left(w^{(k)}\right)$. Dr is a Jacobian matrix and $(D r)_{i j}=\frac{\partial r_{i}}{\partial w_{j}}$.

In the data collection process, the input $\Delta P_{F}$ is swept for $M$ different levels. And for each $\Delta P_{F}$, active laser power $P_{A}$ is swept for $L$ different levels. The $P_{A}$ should be chosen in a way such that the signal magnitude has approximately a square relationship with the effective thermal power $P_{E}$ as discussed before. Finally, the true signal magnitude $S_{T}(t)$ is sampled at $Q$ different time stamps in the interval of $[0.4,4] \mu s$ for each input combination. As a result, the Jacobian matrix becomes a 
$M \times L \times Q$ by 5 matrix since there are 5 unknowns as defined in $w$. Given an initial guess for $w^{(0)}$, the next iteration can be found by $w^{(k+1)}=\left(A^{(k) T} A^{(k)}\right)^{-1} A^{(k) T} b^{(k)}$. Repeat the process until the estimation converges.

Next, the time constant $\tau_{p}$ is swept and the above procedure is repeated until the minimum residue error is obtained.

\subsubsection{Identify Heat Transient Gain and Time Constant}

In this section, the gain $c_{h}$ and time constant $\tau_{h}$ for heat transient process will be identified. The read-back signal magnitude in the time interval $[0,0.1] \mu s$ will be used since the heat transient process dominates in this time frame.

Equation (3.6) shown below governs the whole process of the model. The NFT protrusion can be calculated using the results obtained in the previous section and is denoted as $d_{n m s}(t)$.

$$
f\left(d_{n m s}(t)\right)\left(P_{P L}+\left(c_{h}+\left(1-c_{h}\right)\left(1-e^{-t / \tau_{h}}\right)\right) P_{A}\right)-P_{T h}=S_{T}(t)^{1 / 2} .
$$

Firstly, the gain $c_{h}$ is determined by using data at $t=0$, and (3.6) can be simplified to (3.7).

$$
f\left(d_{n m s}(0)\right)\left(P_{P L}+c_{h} P_{A}\right)-P_{T h}=S_{T}(0)^{1 / 2} .
$$

Define residue function $r\left(c_{h}\right)$ as:

$$
\begin{aligned}
r\left(c_{h}\right) & =f\left(d_{n m s}(0)\right) P_{A} c_{h}+f\left(d_{n m s}(0)\right) P_{P L}-P_{T h}-S_{T}(0)^{1 / 2} \\
& =A c_{h}-b .
\end{aligned}
$$


where $A=f\left(d_{n m s}(0)\right) P_{A}, b=-f\left(d_{n m s}(0)\right) P_{P L}+P_{T h}+S_{T}(0)^{1 / 2}$.

And $c_{h}$ can be found using $c_{h}=\left(A^{T} A\right)^{-1} A^{T} b$.

Next, time constant $\tau_{h}$ is going to be identified. The residue function $r\left(\tau_{h}\right)$ is defined as:

$$
r\left(\tau_{h}\right)=f\left(d_{n m s}(t)\right)\left(P_{P L}+\left(c_{h}+\left(1-c_{h}\right)\left(1-e^{-t / \tau_{h}}\right)\right) P_{A}\right)-P_{T h}-S_{T}(t)^{1 / 2} .
$$

This is again a non-linear least square problem and will be solved using Gaussian-Newton method.

Linearize $r\left(\tau_{h}\right)$ near the current iteration $\tau_{h}^{(k)}$ :

$$
\begin{aligned}
r\left(\tau_{h}\right) & \approx r\left(\tau_{h}^{(k)}\right)+\operatorname{Dr}\left(\tau_{h}^{(k)}\right)\left(\tau_{h}-\tau_{h}^{(k)}\right) \\
& =A^{(k)} \tau_{h}-b^{(k)}
\end{aligned}
$$

where $A^{(k)}=\operatorname{Dr}\left(\tau_{h}^{(k)}\right), b^{(k)}=\operatorname{Dr}\left(\tau_{h}^{(k)}\right) \tau_{h}^{(k)}-r\left(\tau_{h}^{(k)}\right)$.

$D r$ is a Jacobian matrix, where

$$
\begin{aligned}
(D r)_{i} & =\frac{\partial r_{i}}{\partial \tau_{h}} \\
& =-t * f\left(d_{n m s}(t)\right) P_{A}\left(1-c_{h}\right) /\left(\tau_{h}^{2} e^{t / \tau_{h}}\right) .
\end{aligned}
$$

Set initial guess $\tau_{h}^{(0)}=0.1$, the next iteration can be found by $\tau_{h}^{(k+1)}=$ $\left(A^{(k) T} A^{(k)}\right)^{-1} A^{(k) T} b^{(k)}$. Repeat the above procedures until the estimation converges. 


\subsubsection{Identification Results}

In this section, the identified results will be discussed. Firstly, the threshold power $P_{T h}$ is determined by the head and media design. For this parameter, smaller is better because less input laser power is required. And the gains and time constants for NFT fast protrusion and heat transient process are listed in Table 3.2. The time constants for NFT protrusion and heat transient process are indeed in the expected range. The heat transient process gain $c_{h}$ is greater than 0.8 , which shows that most of the temperature increase is completed almost instantaneously.

Table 3.2: Identification Results

\begin{tabular}{|l|l|l|}
\hline \hline Name & Value & Unit \\
\hline$c_{p}$ & 0.27 & $n m / m W$ \\
\hline$\tau_{p}$ & 0.9 & $\mu s$ \\
\hline$c_{h}$ & 0.85 & $N / A$ \\
\hline$\tau_{h}$ & 0.11 & $\mu s$ \\
\hline
\end{tabular}

The identified energy transfer efficiency function $f\left(d_{n m s}\right)$ is plotted in Figure 3.8. The efficiency increases when the NMS decreases, and the trend is very similar to the exponential decay simulated [105].

Since the goal is to minimize the mean square error between the model output and the drive measurement during the identification process, it is desired to compare how good they are matched with each other. The effective thermal power $P_{E}$ is selected for comparison instead of signal magnitude $S_{T}$. If it can be shown that $P_{E}$ is a linearly function of laser power, it can be proved that the square relationship between $P_{E}$ and $S_{T}$ is a good approximation. The drive measurement data and 


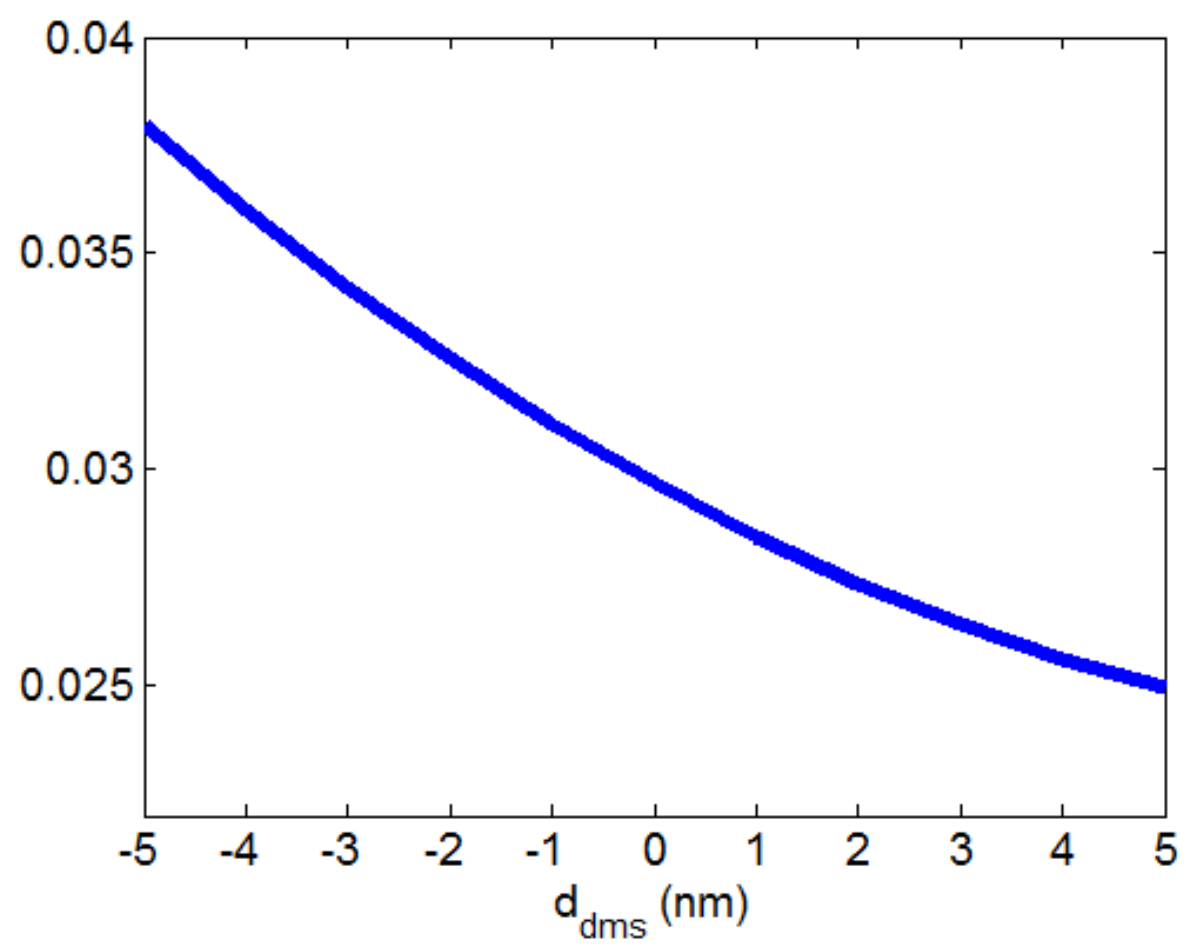

Figure 3.8: Energy transfer efficiency function $f\left(d_{n m s}\right)$.

model output are compared at four time stamps as shown in Figures 3.9 and 3.10 respectively. The comparisons show that they match with each other quite well for all the input conditions.

\subsubsection{Model Verification}

In this section, the accuracy of the identified model are verified by comparing the simulated signal magnitude and that of the real drive measurement data. Since the step laser input current profile is applied during the model identification process, different kind of input profiles must be used for model validation purpose. Different kinds of overshoot will be added on top of the step laser input current change. The overshoot is defined by its OSA and OSD as illustrated in Figure 3.6.

In the first case as shown in Figure 3.11, the default TFC power is retained by setting $\Delta P_{F}$ to 0 . Then the overshoot current of $10 \%$ OSA with two different OSD 


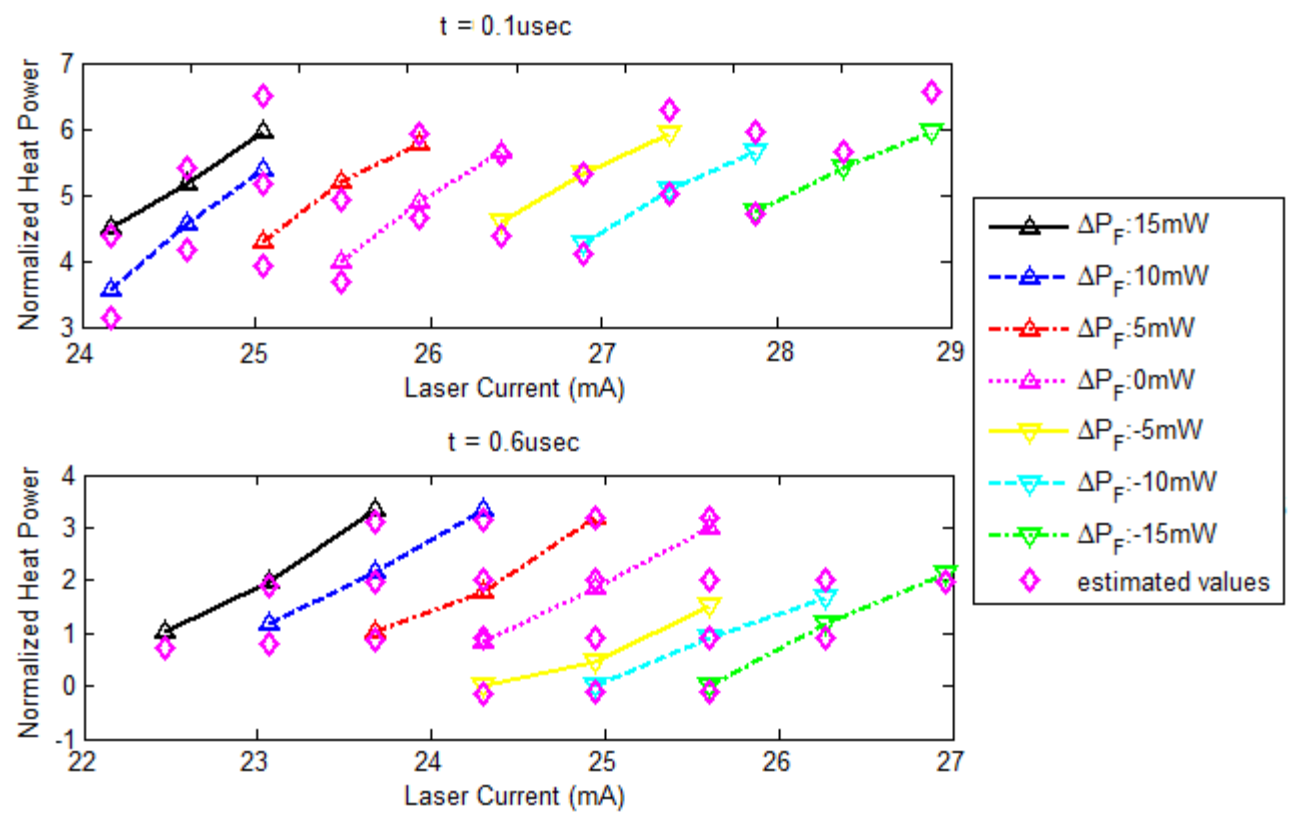

Figure 3.9: Compare $P_{E}$ between drive data and model output at $t=0.1 \mu s$ and $t=0.6 \mu s$.

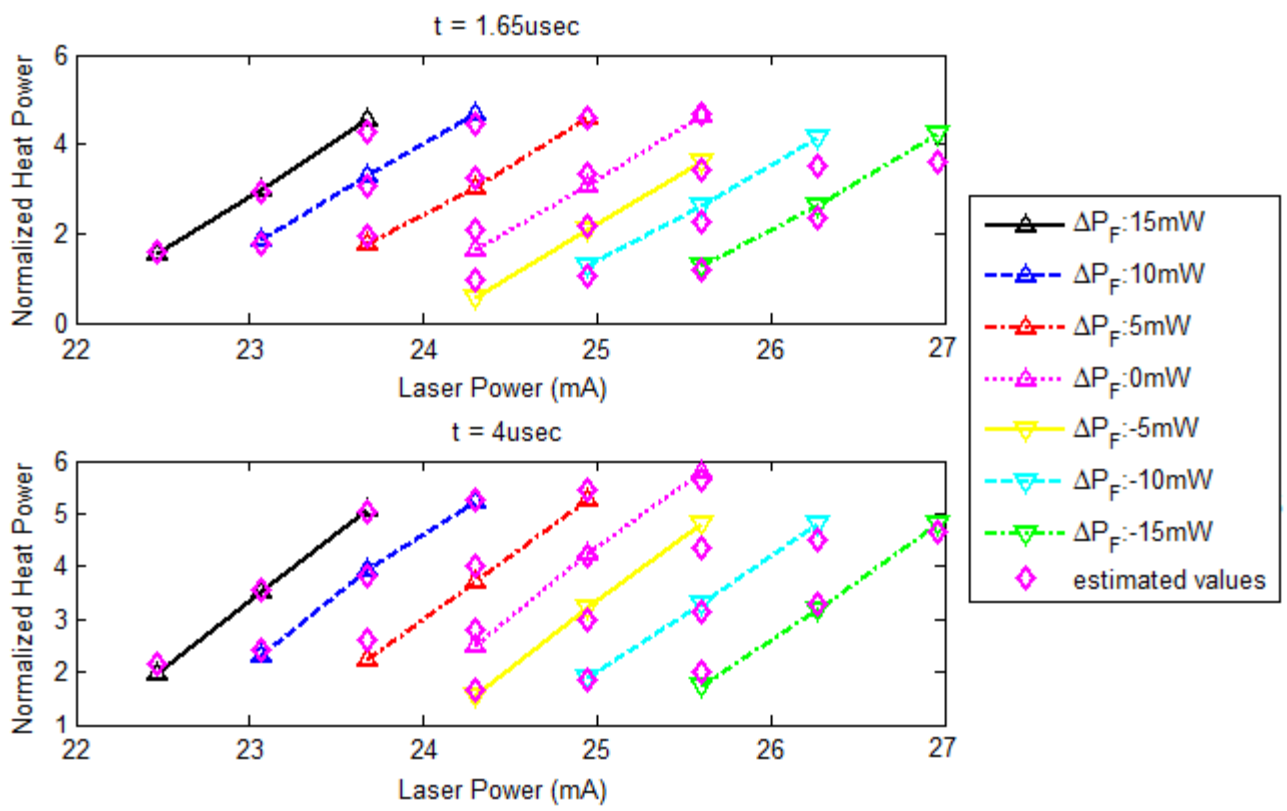

Figure 3.10: Compare $P_{E}$ between drive data and model output at $t=1.65 \mu s$ and $t=4 \mu s$.

settings of 50 and $600 \mathrm{~ns}$ respectively are applied. The input laser current profile $I_{l s r}$ and the predicted NFT fast protrusion amount $d_{p}$ are also plotted in the figure. At the bottom of the figure, the comparison is made on the signal magnitude between 

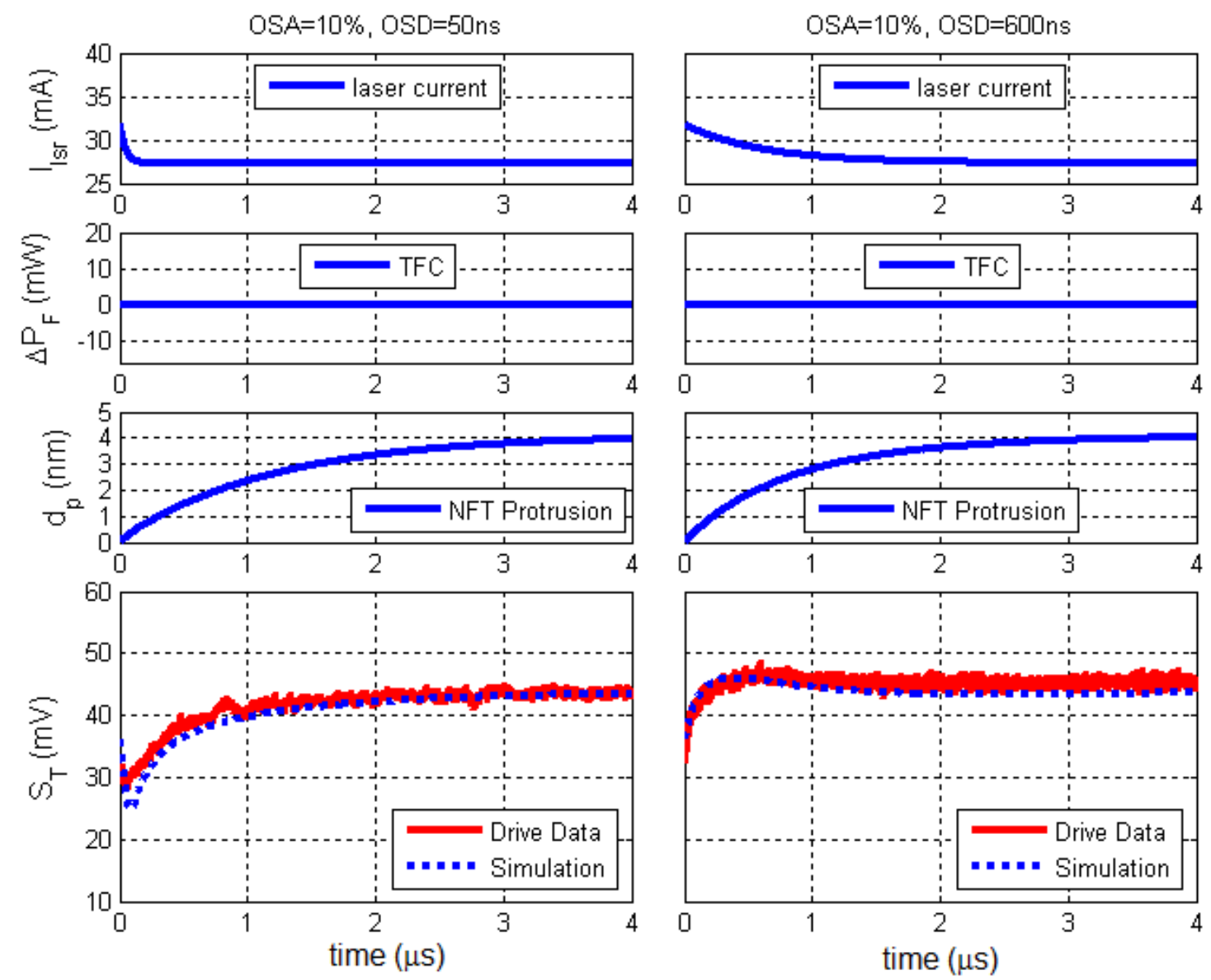

Figure 3.11: Model verification of over-shoot time constants of $50 \mathrm{~ns}$ and $600 \mathrm{~ns}$ with default TFC power.

simulation and measurement data. When OSD is set as $50 \mathrm{~ns}$, the signal magnitude will drop fast in the beginning and then increase slowly. This is due to the very short OSD applied and its effect will diminish very quickly. In contrast, when OSD is set to be $600 \mathrm{~ns}$, there is enough power to increase the signal magnitude for the first $0.5 \mu s$ and then slowly decreases to the steady state. For both of the cases, the simulation results match the measurements quite well.

In Figure 3.12, the fly-height is changed by increasing the heater power by $10 \mathrm{~mW}$. This will make the head fly lower and reduce the NMS. The laser overshoot current OSD is fixed at 350 ns with 10 and $20 \%$ OSA levels. As shown in the figure, the $20 \%$ overshoot current will cause the signal magnitude overshoot at the beginning of 

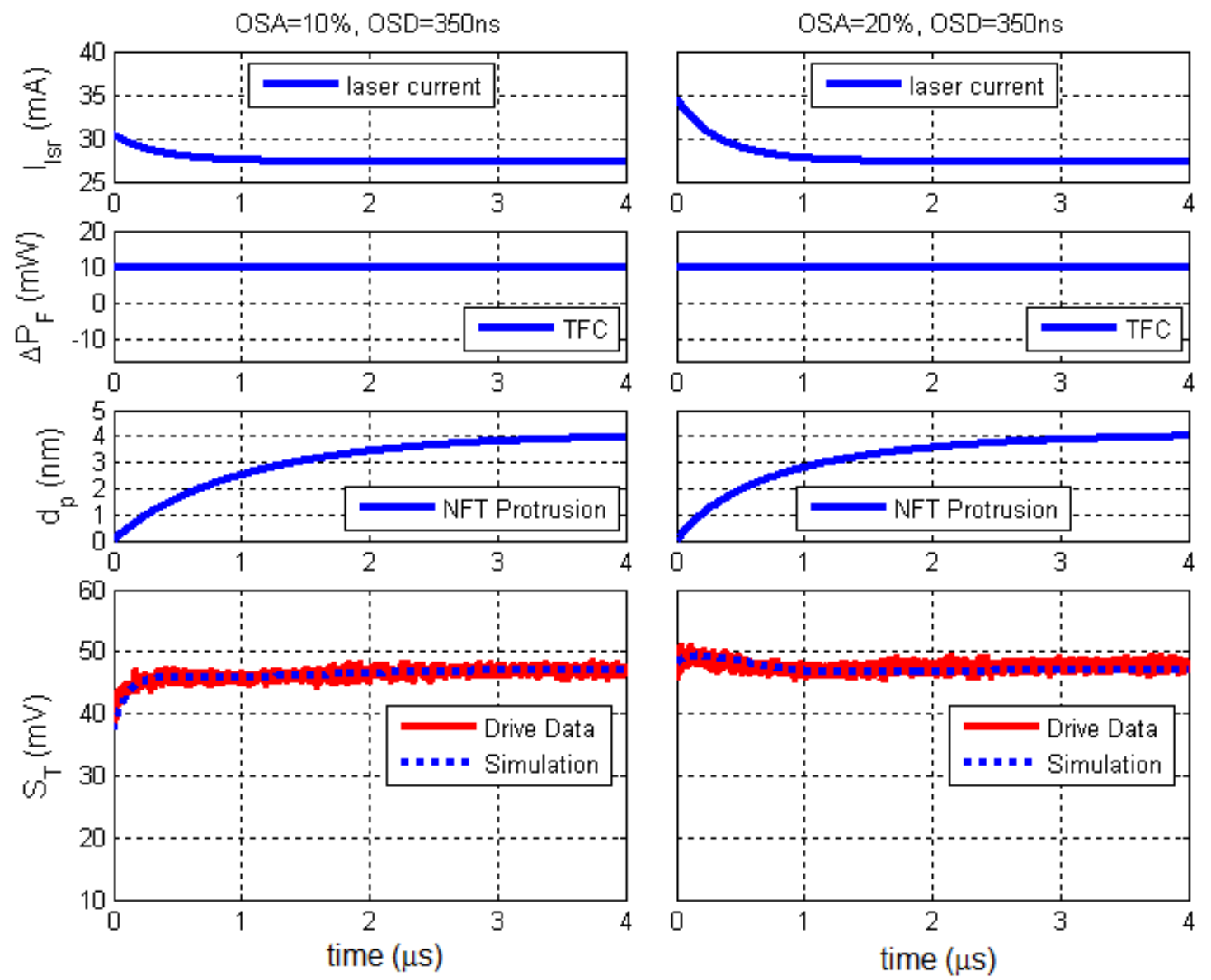

Figure 3.12: Model verification of $10 \%$ and $20 \%$ over-shoot amplitude by increase TFC power by $10 \mathrm{~mW}$.

the writing process. This indicates $20 \%$ overshoot is more than necessary and may over-heat the NFT. Again, the simulation outputs match with drive measurements quite well for all the cases.

In Figure 3.13, the fly-height is increased by decreasing the heater power by $10 \mathrm{~mW}$ while keeping the input current profile the same as the previous test case. Since the NMS is bigger, the signal magnitude will be smaller compared with the previous case since the energy transfer efficiency is lower. The good matching between the simulation and experiment results indicate that the model can predict the energy transfer efficiency at different NMS quite accurately. 

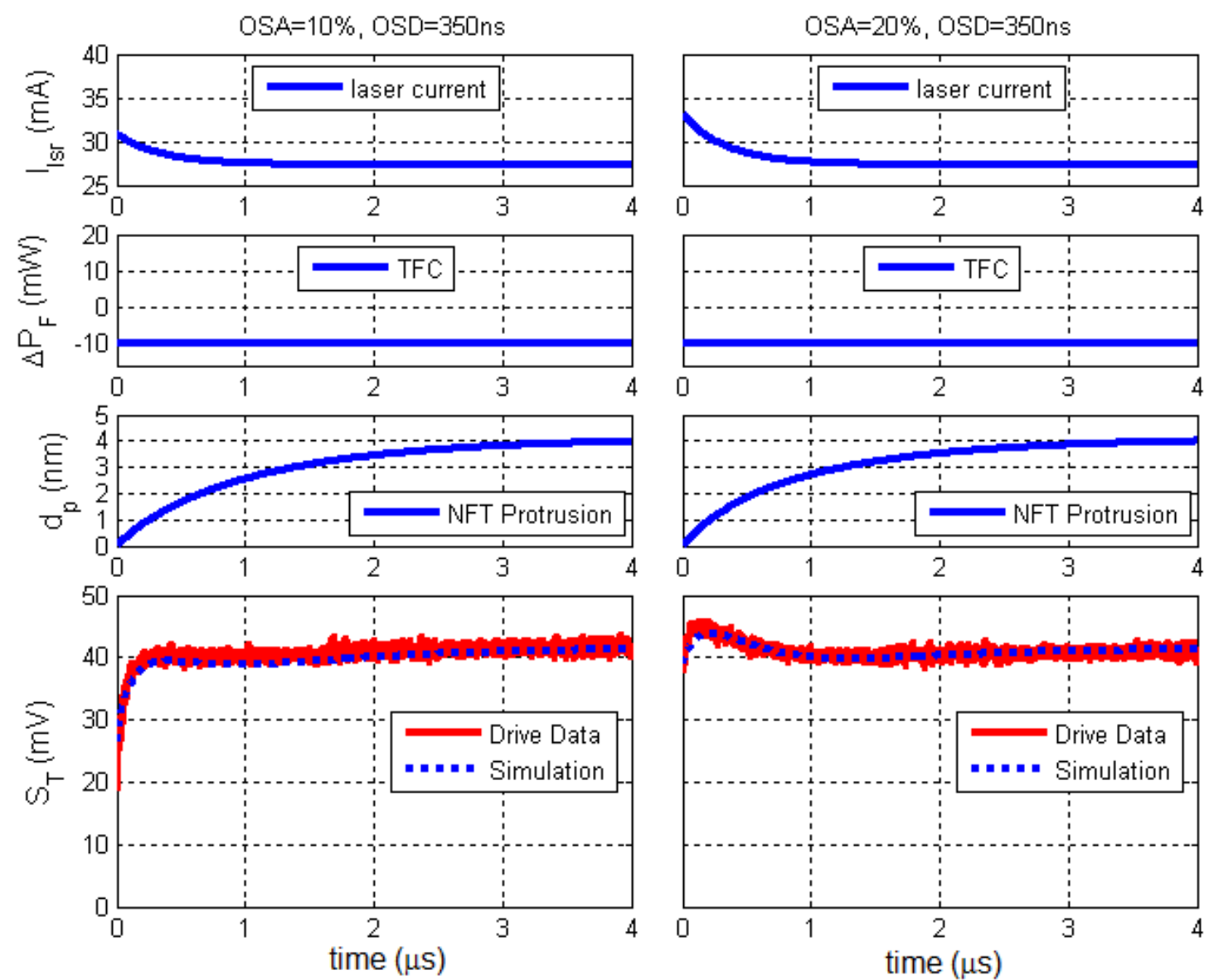

Figure 3.13: Model verification of $10 \%$ and $20 \%$ over-shoot amplitude by decrease TFC power by $10 \mathrm{~mW}$.

\subsection{Slow Protrusion Parameters Identification}

Previously, the NFT fast protrusion and other parameters are identified in the model. In this section, the slow protrusion parameters are going to be identified. Noting that the slow protrusion time constant $\tau_{s}$ is in the order of $100 \mu s$, which is about two orders of magnitude bigger than the fast NFT protrusion time constants $\tau_{p}$, a single tone data pattern of short duration such as $4 \mu s$ will be written on each servo sector of $37.5 \mu \mathrm{s}$. The NFT fast protrusion will reach steady state at the end of this period, but the active laser current induced slow protrusion is very small and can be safely ignored due to the small duty cycle $(\approx 10 \%)$. In this case, the slow 


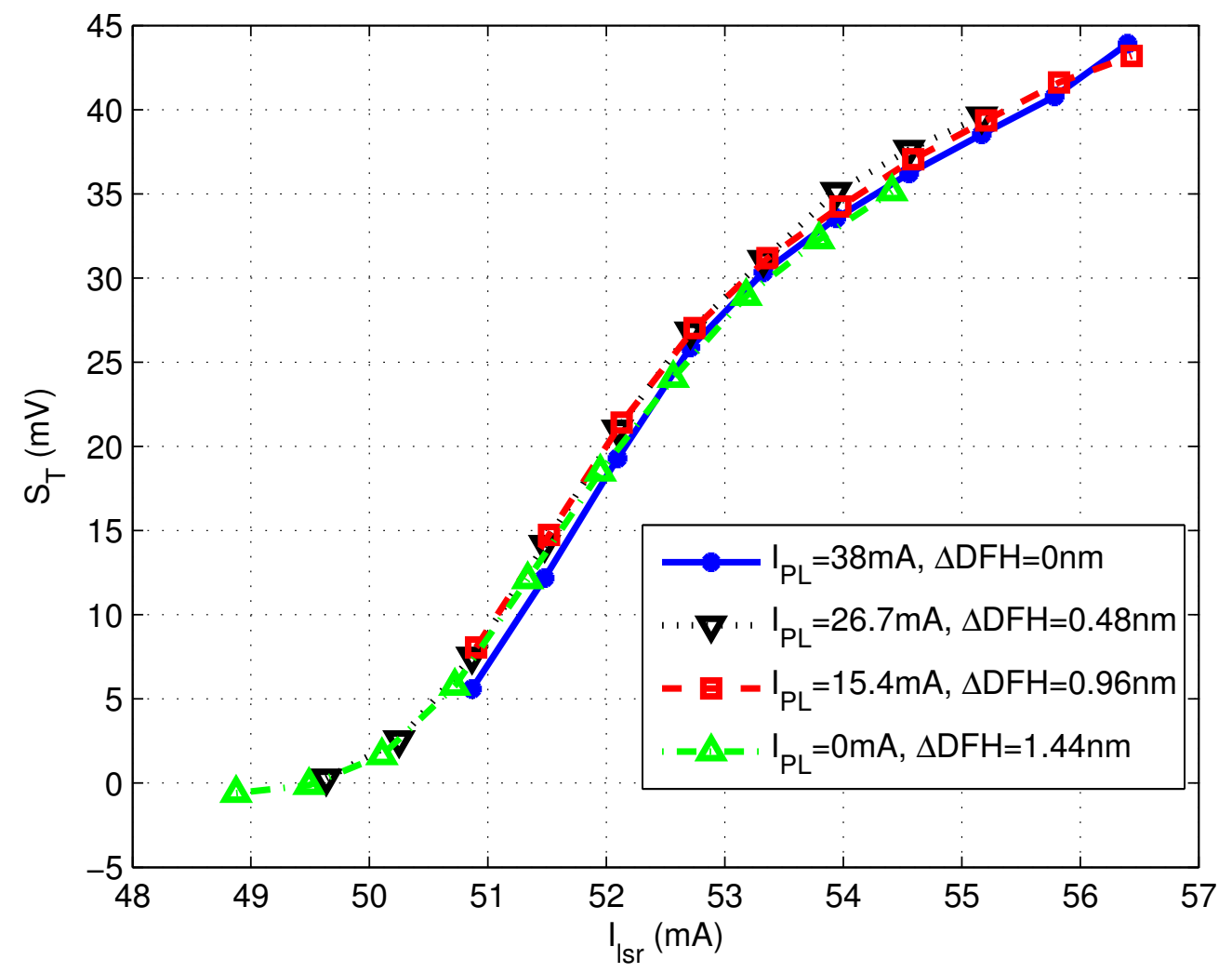

Figure 3.14: Slow protrusion gain measurement.

protrusion is solely generated by the prelase laser current which is applied at $100 \%$ duty cycle. And the steady state of the slow protrusion can be reached after writing for more than 30 sectors consecutively.

In order to find the slow protrusion gain, the prelase current is swept for different levels which will produce different amounts of slow protrusions. For each prelase current level, the active laser current is swept and the signal magnitude is measured at the end of $4 \mu s$ data pattern, where the NFT fast protrusion has completed. Then the signal magnitude $S_{T}$ is calculated by averaging the measured signal magnitude for all the sectors except the first 30 sectors, where the slow protrusion has not reached its steady state. The signal magnitude $S_{T}$ versus total laser current $I_{l s r}$ can be plotted for each prelase current level, where total laser current is the sum of the prelase and active laser current. Different prelase current will produce different slow 


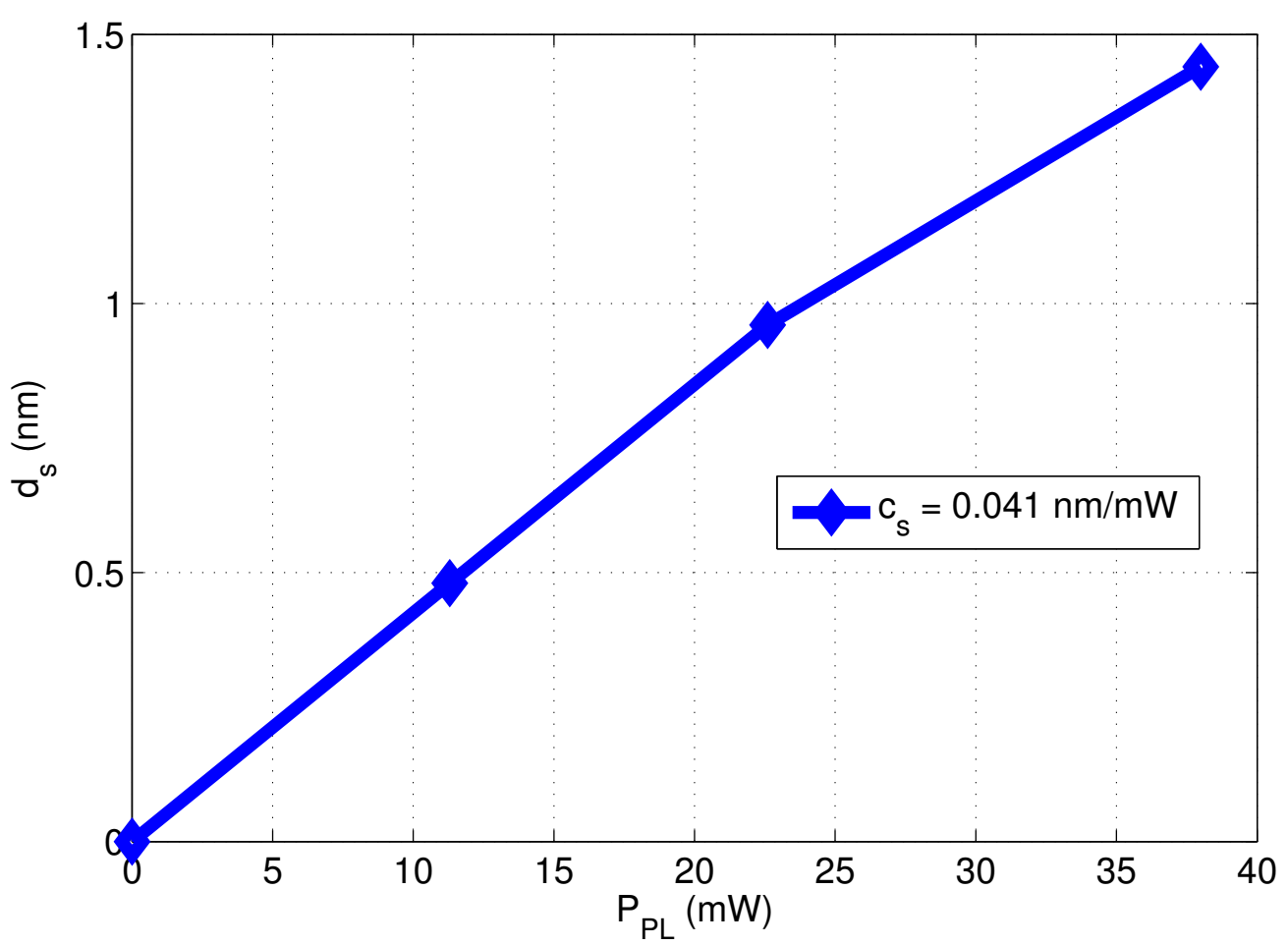

Figure 3.15: Slow protrusion vs. laser power.

protrusion, but the NFT fast protrusion will remain the same as long as the total laser current is the same. As a result, smaller prelase current will produce smaller written-in signal magnitude given the same total laser current and constant TFC power.

In order to compensate for this fly-height difference caused by the different prelase current induced slow protrusion, the TFC power can be increased in the case of the smaller prelase current until the curves overlap with each other as shown in Figure 3.14. Since the total laser power is the same, and written-in signal magnitude is the same, it is necessary that the adjusted TFC flying-height has compensated for the difference caused by the slow protrusion. By extracting the data from Figure 3.14, the slow protrusion in $n m$ versus the prelase laser power $P_{P L}$ is plotted as shown in Figure 3.15. The relationship is quite linear and the gain $c_{s} \approx 0.041$ $n m / m W$. 


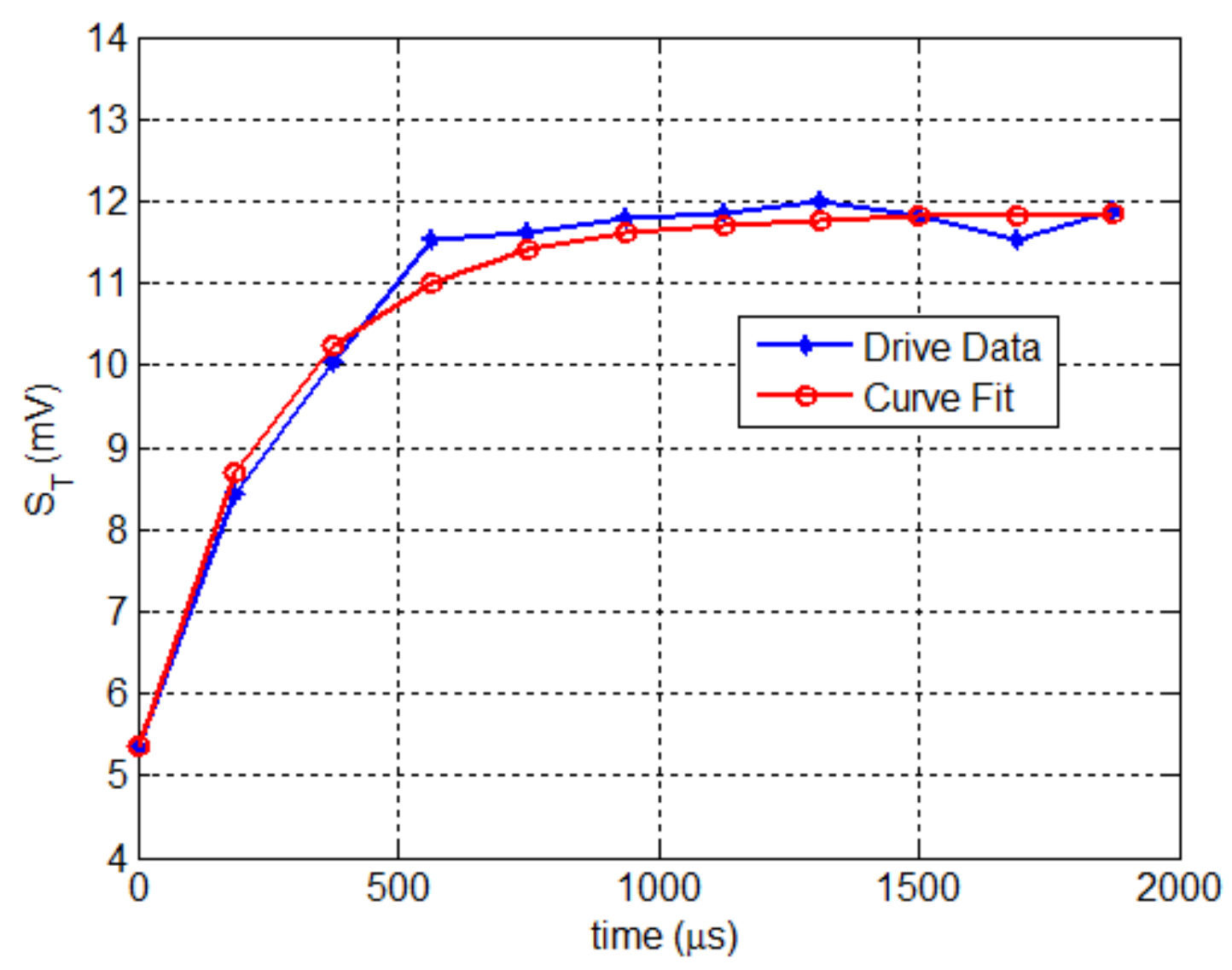

Figure 3.16: Slow protrusion time constant measurement.

To measure the time constant of this slow protrusion, the writing-start wedge is swept from 0 to 50 and a data pattern with a maximum duty cycle $(\approx 90 \%)$ is written. Then the signal magnitude is measured at wedge \#50. Since the laser power is applied at the first writing wedge only, the amount of protrusion will be determined by the time taken from the first wedge to wedge \#50. The signal magnitude versus time is plotted in Figure 3.16 together with the curve fitted one. In order to find the time constant of the protrusion, the signal magnitude in Figure 3.16 needs to be converted to the protrusion as shown in Figure 3.17 by using $g\left(P_{E}\right)$ in our model for back calculation. The slow protrusion time constant $\tau_{s}$ is found to be around $400 \mu s$ in our case. 


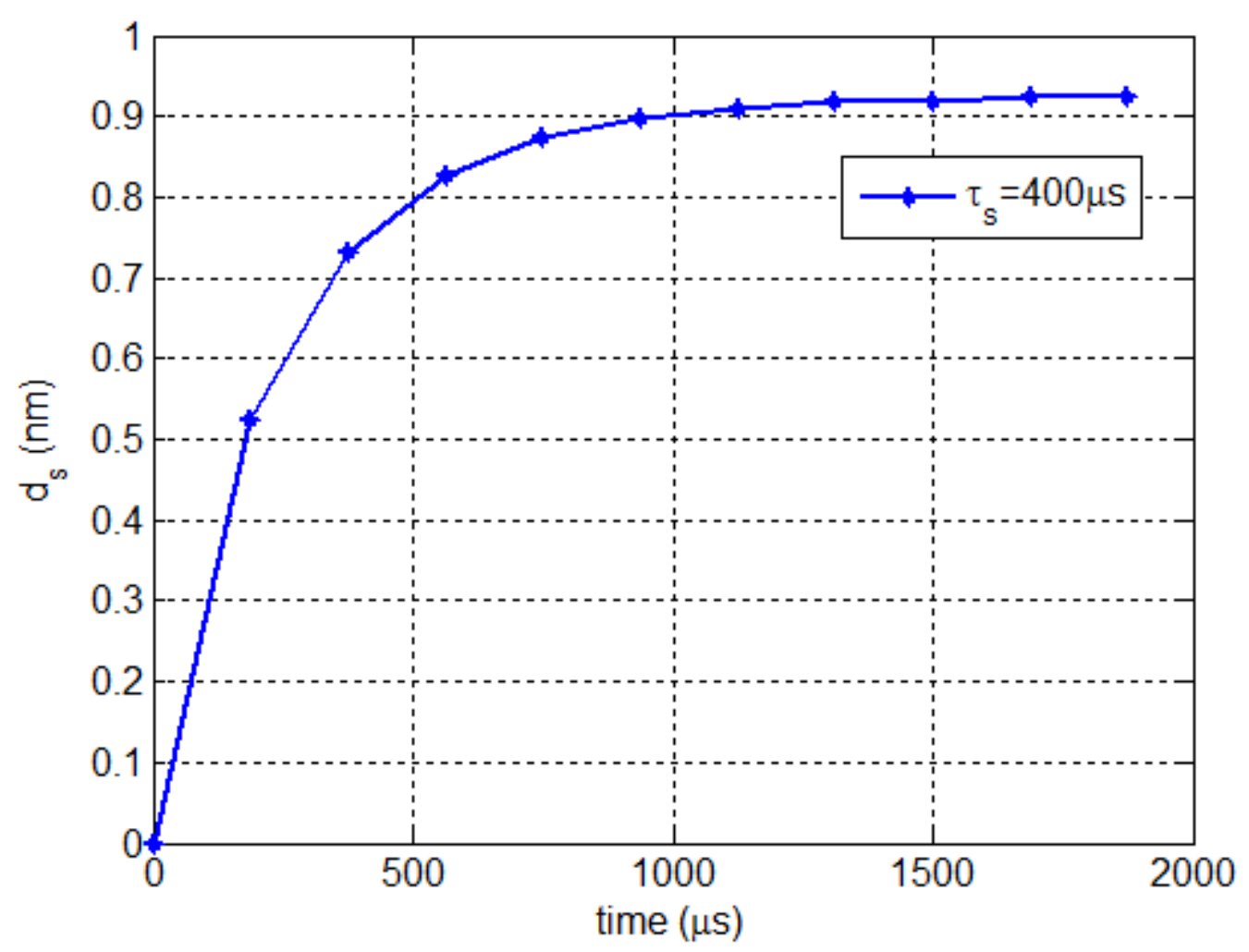

Figure 3.17: Slow protrusion vs. time.

\subsection{Conclusion}

This chapter presented how to construct the HAMR writing model with some approximations and assumptions based on prior knowledge. Model parameters are identified such as the gains and time constants related to heat transient process, NFT protrusion, and slow protrusion respectively. The energy transfer efficiency function and the threshold thermal power are also identified. Since the system is non-linear, the Gaussian-Newton method is used for the identification. The model is quite accurate by comparing the simulation output and drive data for different input conditions. Another very important aspect of this model is that it can predict the NFT protrusion, which is not measurable in drive operation. This will enable us to optimize the fly-height control in data writing process. In the next chapter, applications of this model will be discussed in more detail. 



\section{Chapter 4}

\section{Applications for HAMR Writing Model}

\subsection{Introduction}

In the previous chapter, the HAMR writing model is constructed and identified, which includes slow protrusion, fast NFT protrusion, energy transfer efficiency, heat transient process, and power-to-signal magnitude conversion. Heat transient process, NFT fast protrusion and the slow protrusion are the three dynamics involved in the writing process. This model can be used to simulate the signal output and NMS change under different input conditions: input laser power profile and initial NMS.

In this chapter, two applications of this model will be discussed as shown in the following sections. In Section 4.2, discussion will be made on how to keep NMS closest to, but not below its target value across multiple servo wedges or data sectors throughout the writing process. Three writing conditions of different duty cycle will 
be considered: repeatable run-out (RRO) field write, servo pattern write and user data field write. Firstly, it will be formulated on how to calculate the slow and NFT protrusion amount at each servo wedge or data sector with respect to the writing duty cycle. The laser induced protrusions will change the NMS and need to be compensated. Next, it will be shown on how to compensate for the NMS change by using the thermal fly-height control (TFC). In Section 4.3, the compensation of the impacts caused by the fast transients will be demonstrated, which include NFT protrusion and heat transient. The fast transients will cause bad writtenin signal quality (low signal amplitude) at the beginning of each writing process when the write gate is turned on. Two methods, pole placement and deconvolution method, are presented to alleviate the fast transient impacts by shaping the input laser current profiles.

\subsection{Compensation of Protrusion Caused NMS Change}

In this section, the goal is to minimize NMS error relative to its target spacing during the writing process to improve the written-in signal quality and minimize the input laser power to prevent NFT from over-heating so that its lifetime can be prolonged. Firstly, the equations to calculate the fast NFT and slow protrusions will be derived under different writing duty cycles. Next, it will be shown on how to use the TFC to compensate for these NMS changes.

\subsubsection{Calculate NFT and Slow Protrusions}

As discussed in Chapter 3, the tiny sized NFT protrudes fast with a gain of $c_{p}$ and time constant of $\tau_{p}$. NFT will start to protrude when the input laser power is 


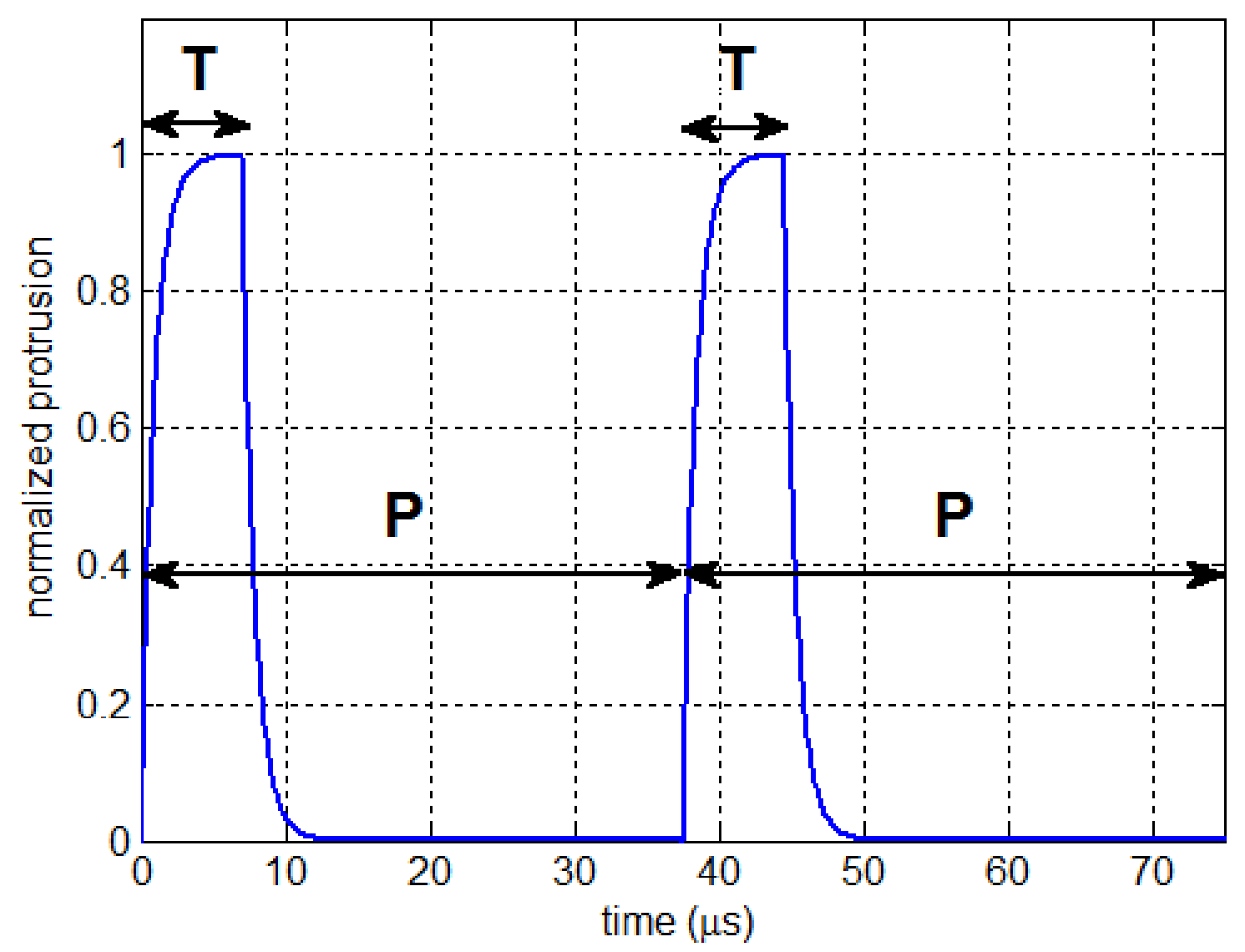

Figure 4.1: Illustration of normalized NFT protrusion and decay. $P$ is the servo sampling time. $T$ is the write gate turned on time for each servo wedge or data sector.

applied during the writing process for the time interval from 0 to $T$ as illustrated in Figure 4.1. When the input laser power is removed at the end of $T$ till the end of $P$, the protrusion starts to decay with a time constant $\tau_{p}^{\prime}$, where $P$ is the servo sampling time. In the remainder of this chapter, it is assumed that both the protrusion and decay processes have the same time constant with $\tau_{p}^{\prime}=\tau_{p}$ unless otherwise stated.

For a write operation lasting for $N$ servo wedges or data sectors, the NFT protrusion amount at the end of the first wedge or sector will be:

$$
d_{p_{-} 1}=P_{A} c_{p}\left(1-e^{-\frac{T}{\tau_{p}}}\right) .
$$


The accumulated NFT protrusion amount at the end of the second wedge or sector including decay process will be:

$$
\begin{aligned}
& d_{p \_2}=P_{A}\left[c_{p}\left(1-e^{-\frac{T}{\tau_{p}}}\right) e^{-\frac{P}{\tau_{p}^{\prime}}}+c_{p}\left(1-e^{-\frac{T}{\tau_{p}}}\right)\right] \\
& \quad=P_{A}\left[c_{p}\left(1-e^{-\frac{T}{\tau_{p}}}\right) e^{-\frac{P}{\tau_{p}}}+c_{p}\left(1-e^{-\frac{T}{\tau_{p}}}\right)\right], \\
& \quad \text { where } \tau_{p}^{\prime}=\tau_{p} .
\end{aligned}
$$

At the end of $N$ wedges or sectors of consecutive writing, the accumulated protrusion amount can be expressed as:

$$
\begin{aligned}
d_{p_{-} N} & =P_{A}\left[c_{p}\left(1-e^{-\frac{T}{\tau_{p}}}\right) e^{-\frac{(N-1) P}{\tau_{p}}}+c_{p}\left(1-e^{-\frac{T}{\tau_{p}}}\right) e^{-\frac{(N-2) P}{\tau_{p}}}+\cdots+c_{p}\left(1-e^{-\frac{T}{\tau_{p}}}\right)\right] \\
& =P_{A} \sum_{k=0}^{N-1} c_{p}\left(1-e^{-\frac{T}{\tau_{p}}}\right) e^{-\frac{k P}{\tau_{p}}}
\end{aligned}
$$

The slow protrusion can be calculated in a similar manner, where $c_{s}$ and $\tau_{s}$ represent the gain and time constant respectively. It is also assumed that the time constant for decay processes $\tau_{s}^{\prime}$ is the same as the protrusion time constant $\tau_{s}$ in the remainder of this chapter unless otherwise stated. Then, the slow protrusion amount across multiple servo wedges or data sectors in a writing process can be written as:

$$
d_{s_{-} N}=P_{A} \sum_{k=0}^{N-1} c_{s}\left(1-e^{-\frac{T}{\tau_{s}}}\right) e^{-\frac{k P}{\tau_{s}}}
$$

The duty cycle of the actual writing plays an important role in HAMR writing optimization. The duty cycle $D$ is expressed as:

$$
D=\frac{T}{P} \cdot 100 \%
$$

Substituting equation (4.5) into equations (4.3) and (4.4), the NFT and slow 
protrusions can be calculated based on different duty cycle for different types of writing as:

$$
\begin{aligned}
& d_{p_{-} N}=P_{A} c_{p}\left(1-e^{-\frac{D P}{\tau_{p}}}\right) \sum_{k=0}^{N-1} e^{-\frac{k P}{\tau_{p}}} . \\
& d_{s_{-} N}=P_{A} c_{s}\left(1-e^{-\frac{D P}{\tau_{s}}}\right) \sum_{k=0}^{N-1} e^{-\frac{k P}{\tau_{s}}}
\end{aligned}
$$

The total protrusion will increase with higher duty cycle $D$ and the larger number of servo wedges or data sectors $N$.

\subsubsection{RRO Field Write, Servo Pattern Write and User Data Field Write}

The servo sampling time in a 2.5" $5400 \mathrm{rpm}$ HAMR drive used in the experiment is around $37.5 \mu \mathrm{s}$, and the writing of RRO field takes about $0.6 \mu s$ for each servo wedge. The corresponding duty cycle for RRO field write is around $1.6 \%$. For comparison, the writing duty cycle for servo pattern and user data are around 5\% and $92 \%$ respectively in our test case [157].

In Chapter 3, the identified NFT protrusion time constant is about $\tau_{p}=0.75$ $\mu s$, slow protrusion time constant is around $\tau_{s}=400 \mu s$ for the pattern that was considered. For RRO field write, with $P=37.5 \mu s, D=1.6 \%$, the NFT protrusion for the first wedge is around $0.55 P_{A} c_{p}$ and the slow protrusion is about $0.0015 P_{A} c_{s}$ based on equations (4.6) and (4.7) respectively. At the last servo wedge of a full track RRO field write, the NFT protrusion is still around $0.55 P_{A} c_{p}$ although the slow protrusion increases to about $0.017 P_{A} c_{s}$.

For servo pattern write, with $D=5 \%$, the NFT protrusion at the first wedge is about $0.92 P_{A} c_{p}$ and the slow protrusion is around $0.0047 P_{A} c_{s}$. At the last wedge 


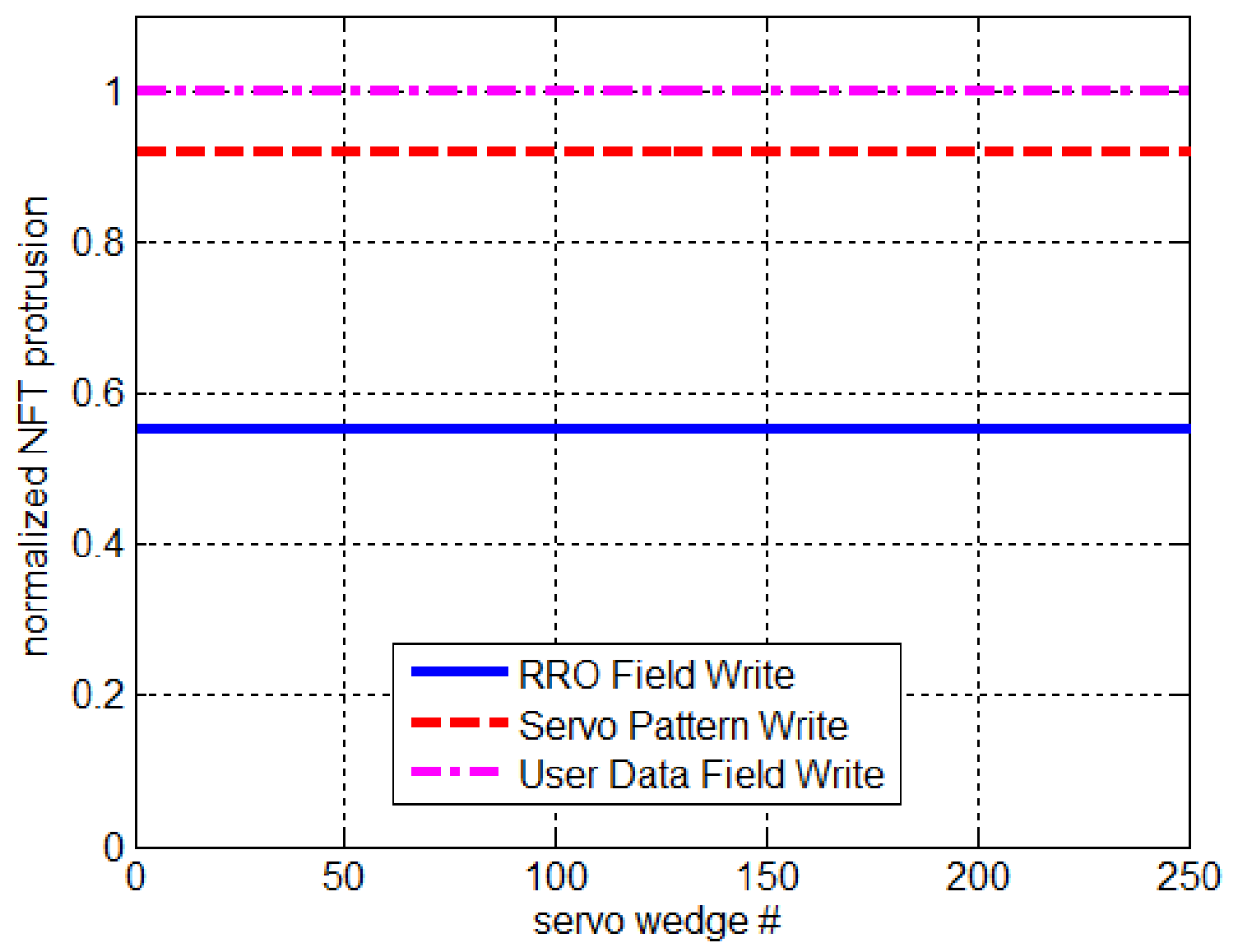

Figure 4.2: Normalized fast NFT protrusion amount at the end of writing for each servo wedge or data sector. Comparisons are made among RRO field write, servo pattern write and user data field write.

$\mathrm{N}$, the NFT protrusion is still around $0.92 P_{A} c_{p}$ and the slow protrusion is increased to $0.052 P_{A} c_{s}$.

For user data field write of same frequency pattern, with $D=92 \%$, the NFT protrusion at the first sector is $P_{A} c_{p}$ and the slow protrusion is around $0.083 P_{A} c_{s}$. At the last sector $\mathrm{N}$ of user data writing, the NFT protrusion is still around $P_{A} c_{p}$ and the slow protrusion is increased to about $0.92 P_{A} c_{s}$.

The NFT and slow protrusions amount over multiple servo wedges or data sectors for RRO field write, servo pattern write and user data write are compared in Figures 4.2 and 4.3 respectively. 


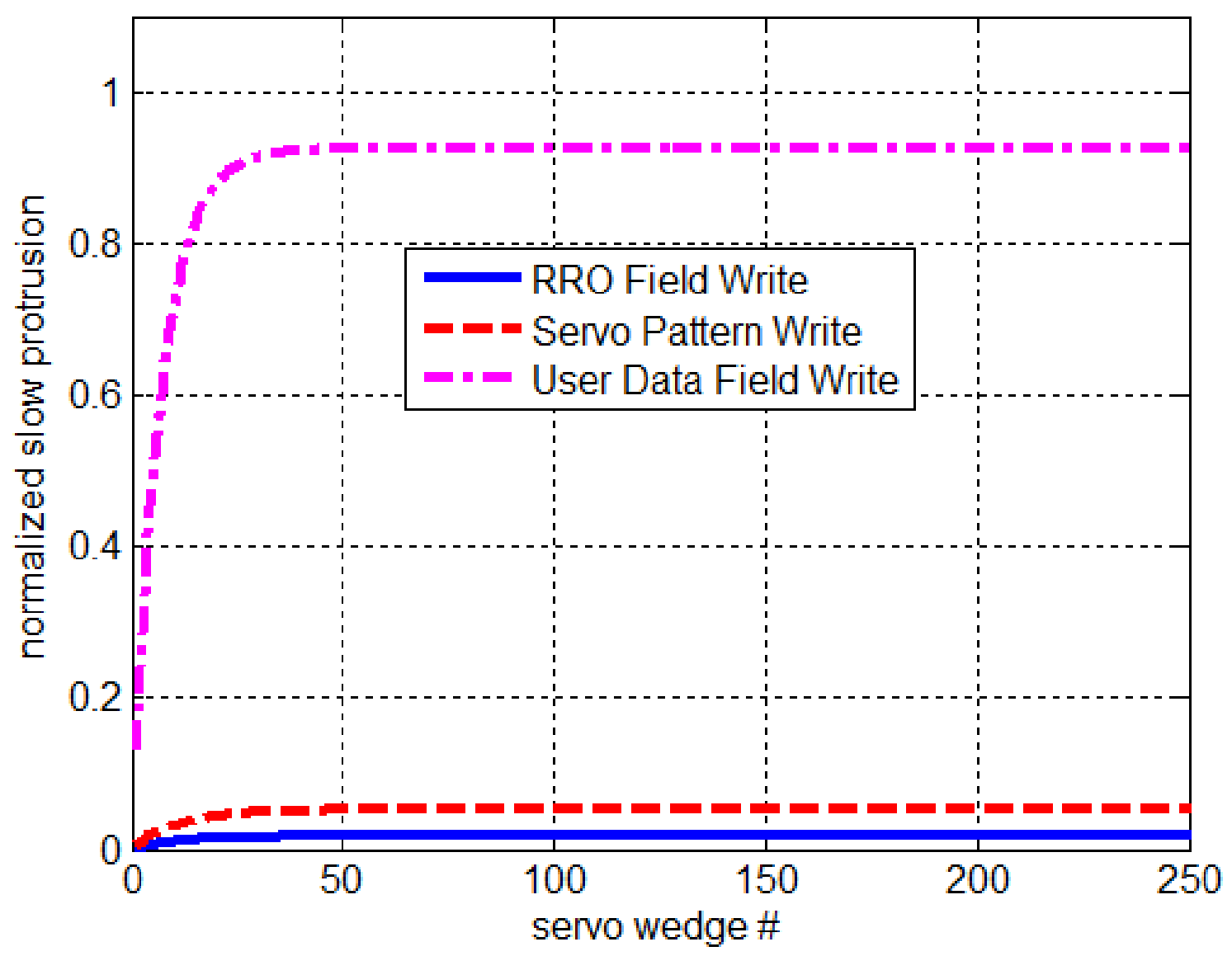

Figure 4.3: Normalized slow protrusion amount at the end of writing for each servo wedge or data sector. Comparisons are made among RRO field write, servo pattern write and user data field write.

\subsubsection{Compensation of NFT Protrusion Caused NMS Change}

As shown in Figure 4.2, for each writing condition, the NFT protrusion amount across the servo wedges or data sectors during the writing process is constant. As a result, there is no dynamic compensation required. However, there are steady state protrusion amount differences due to different writing duty cycles. These differences should be addressed and compensated, so that the NMS is kept close to the target value for all the writing conditions.

The most effective and cost-free method to compensate for the slow protrusion 
caused NMS change is to use TFC, which is well developed and understood in conventional drives [158]. By controlling the power delivered to the heater, the fly-height of the write head, and hence the NMS can be changed as desired.

\subsubsection{Compensation of Slow Protrusion Caused NMS Change}

Figure 4.3 shows the slow protrusion across servo wedges or data sectors for all the three writing conditions. Since the impacts of the slow protrusion on RRO field writing and servo pattern writing are small due to their low writing duty cycles, the following discussion will be made on how to compensate for user data field writing only.

It is assumed that the TFC control can be modeled as a first order system with the time constant $\tau_{f}$, which needs to be less than or equal to the slow protrusion time constant $\tau_{s}$ in order to achieve the best compensation results. Let $h_{f}[n]$ be the unit sample response of the TFC control in discrete time, which can be calibrated and is known to us in advance. The slow protrusion will cause the reduction of NMS and is represented by $y_{s}[n]$, which is normalized and shown in Figure 4.4 (b). In order to compensate for this NMS reduction, the heater power needs to be reduced to increase the NMS for the same amount. The desired heater power can be found by using the deconvolution method [159] through equation (4.8), where TFC induced NMS change is denoted as $y_{f}[n]$, where $y_{f}[n]=-y_{s}[n]$ as shown in Figure $4.4(\mathrm{~b})$. In this thesis, deconvolution is performed using the deconv function, that is available in MATLAB.

$$
u_{f}[n]=-\operatorname{deconv}\left(y_{f}[n], h_{f}[n]\right) .
$$

The derived TFC heater power reduction profile $u_{f}[n]$ is shown in Figure 4.4 (a) and the net NMS change is very small after compensation. The result is shown 

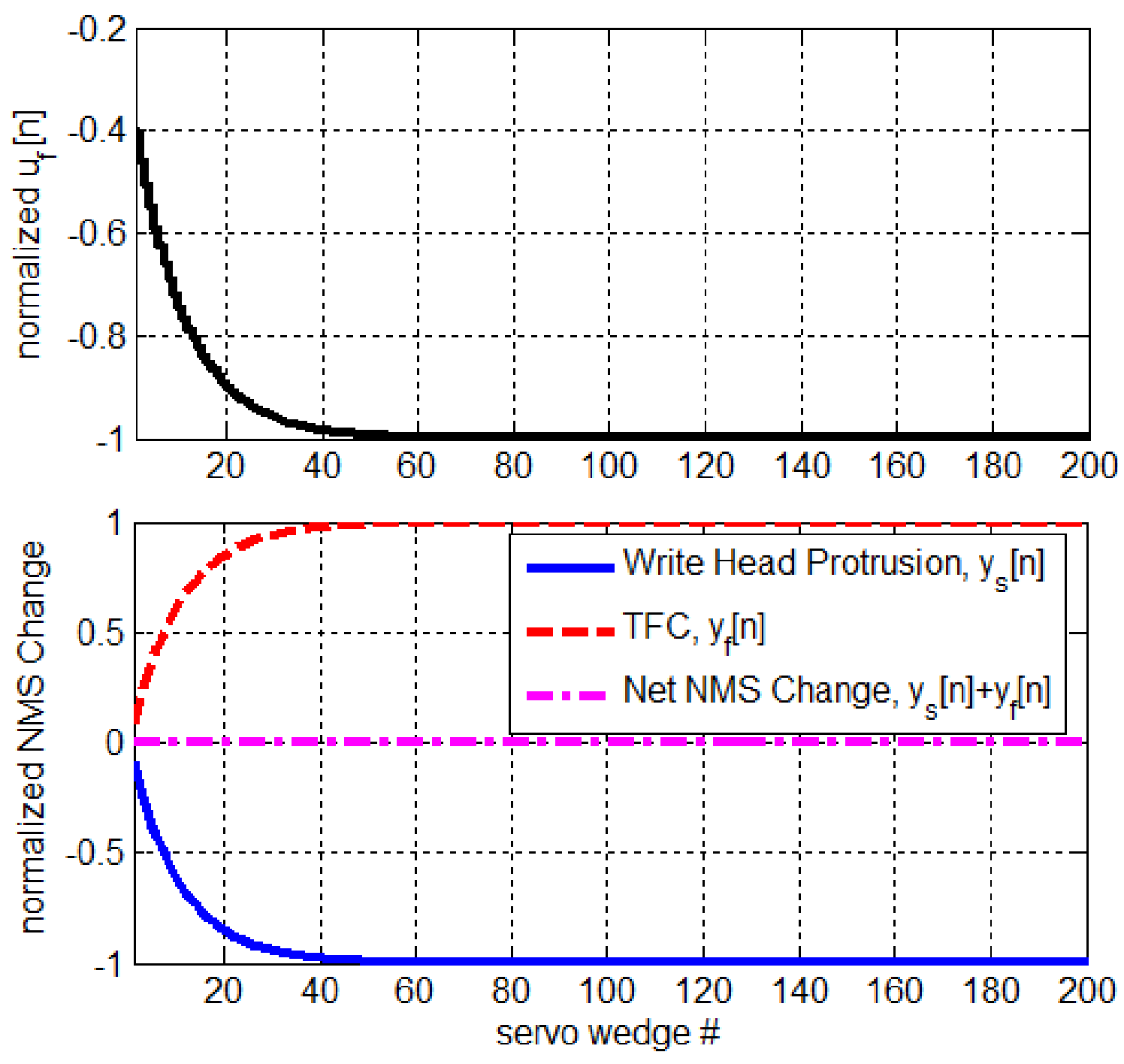

Figure 4.4: Using TFC to compensate for the slow protrusion. (a) Heater power profile. Negative value means the heater power is reduced from initial value; (b) slow protrusion will cause the NMS to decrease whereas the reduction of TFC heater power will cause the NMS to increase. The net NMS change is approaching to 0.

in Figure 4.4 (b).

\subsection{Compensation of Fast Transient Impact}

In this section, the compensation of the fast transient impacts will be discussed, which includes NFT protrusion and heat transient. The signal quality is bad for the 
first few microseconds each time when the write gate is turned on for writing if no compensation is applied as shown in the black curve (no overshoot case) of Figure 4.9. This kind of signal degradation is not desired for RRO field and user data write. If not compensated, a long preamble needs to be added in front of the useful data and the format efficiency will be reduced. However, this fast transient impact is not a problem for servo pattern write. The reason is that the servo pattern is written in the self servo write process, and the written-in long preamble can be erased in the following process so that there is no format efficiency loss incurred.

The fast transient of HAMR writing must be compensated on a per wedge or sector basis. Just like PMR drive, in order to compensate the transient, which happens within a few hundred picoseconds from turning on the magnetic write field [160], the exponential decay over-shoot in writing current is added to the writer coil as illustrated in Figure 4.5. The similar idea is also implemented for HAMR drive by adding an overshoot circuit to pre-amplifier to produce extra laser current with an exponential decay time constant to compensate for the transient effects. The hardware provides options to adjust the overshoot amplitude (OSA) and the exponential decay time constant, which is also called overshoot duration (OSD). The optimal overshoot settings are normally obtained by time-consuming sweeping method in the calibration process. In the following two subsections, pole placement and deconvolution methods will be presented, which are used to find the optimized input laser current profiles.

\subsubsection{Laser Current Overshoot Design via Pole Placement Method}

In this section, the optimized overshoot settings will be derived by using the pole placement method in a systematic way with the established model. Our goal 

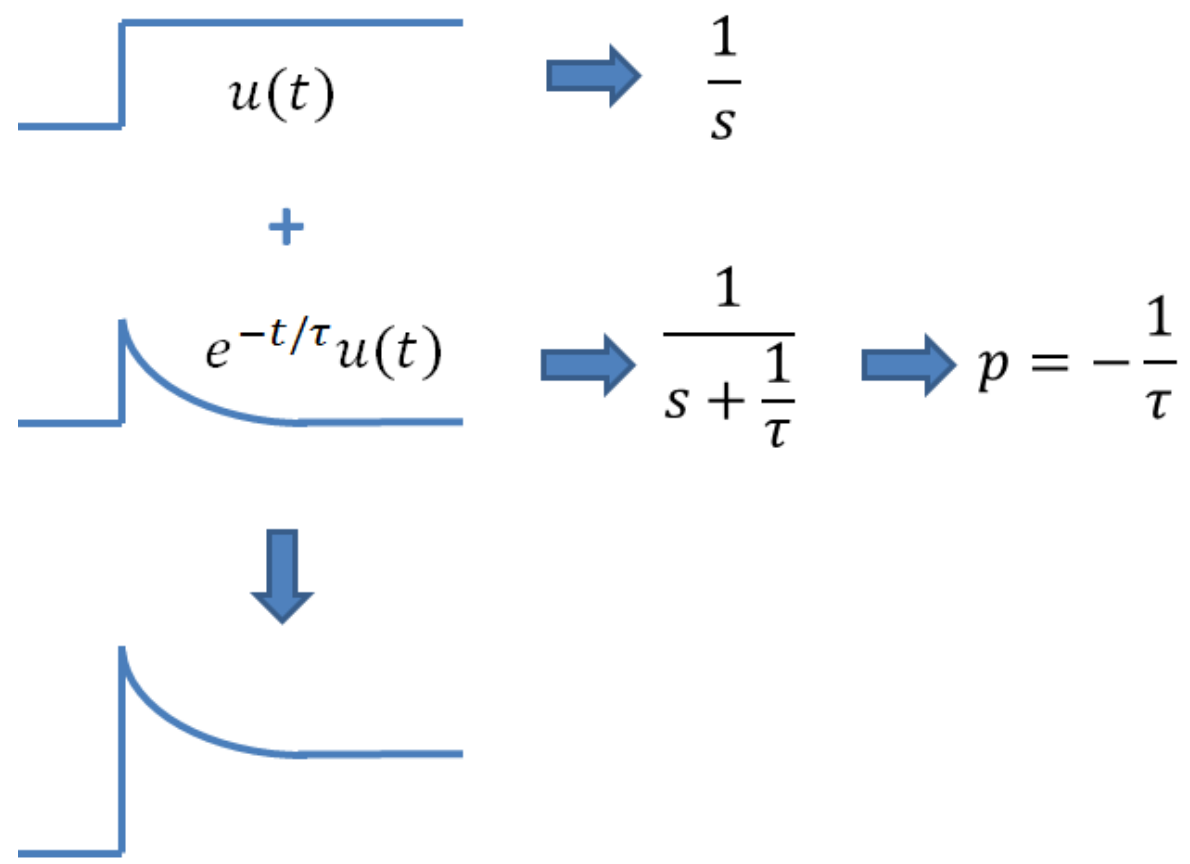

Figure 4.5: Illustration of exponential decay overshoot components added on top of the step input and their Laplace transform.

is to shape the input laser current to achieve a step change in the written-in signal magnitude at the output. In other words, the output signal magnitude $S_{T}$ has a constant value from the very beginning of the write gate until the end. Since the power-to-signal magnitude conversion function $g\left(P_{M}\right)$ is a static function, the constant signal magnitude $S_{T}$ can be achieved if and only if the media thermal power $P_{M}$ is constant during the writing process. As a result, the signal magnitude $S_{T}$ and power-to-signal magnitude conversion function $g\left(P_{M}\right)$ will be excluded from the model for the following discussion to simplify the analysis. In addition, since the input of our model is laser power, the optimized laser power will be found first and then converted to laser current later on. The simplified model is shown in Figure 4.6 .

Previous study in Chapter 3 shows that the system $h(t)$ is a non-linear system. In order to derive the desired input profile using the pole placement method, the system needs to be linearized. First, the slow protrusion portion is removed from 


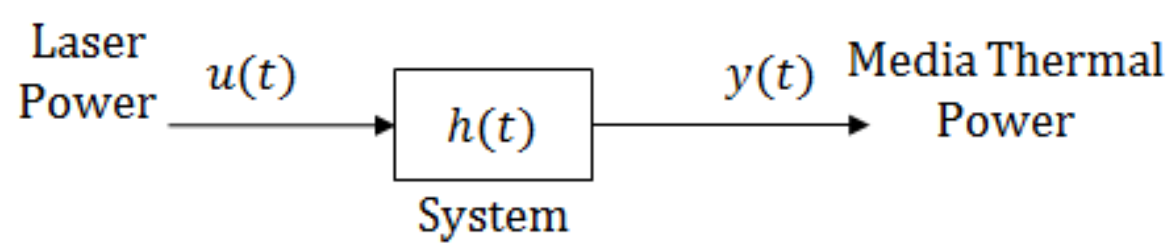

Figure 4.6: Simplified model with laser power as input and media thermal power as output.

the model since it is not relevant in this study due to its slow dynamic. Next, the energy transfer efficiency function $f\left(d_{n m s}\right)$ can be approximated by a first order polynomial function or affine function rather than second order polynomial used in Chapter 3. This approximation produces good results since the NFT protrusion amount is in a small range only. It is also assumed that the magnitude of the input overshoot component is only a small portion of the total laser current. Finally, with some simplifications, the original system can be linearized and its transfer function can be expressed as:

$$
H(s)=c_{a}\left(c_{b}+\frac{1-c_{b}}{\tau_{b} s+1}\right)\left(c_{c}+\frac{1-c_{c}}{\tau_{c} s+1}\right) .
$$

In equation (4.9), different symbols are used for the gains and time constants from those used in the model since they are not exactly the same as the ones from the original non-linear model. The parameters of the linearized system need to be identified first. And the identification can be done using the similar method as discussed in Chapter 3.

Given the linearized second order model by equation (4.9), the Laplace transform of the input laser power $U(s)$ is proposed to be:

$$
U(s)=\frac{P_{0}}{s}+\frac{P_{1}}{s+1 / \tau_{1}}+\frac{P_{2}}{s+1 / \tau_{2}} .
$$

The first item of equation (4.10) is the step input laser power without any 
overshoot and the second and third items define the exponential decay overshoot components. The basic idea is to use two overshoot components to cancel the two dynamics of the system respectively.

In the ideal case, a step change in the output is desired so that the transient impacts can be completely eliminated. The Laplace transform of a step function of the output is defined in equation (4.11), where $P_{M}$ is a constant and presents the target thermal power delivered to the media.

$$
Y(s)=\frac{P_{M}}{s}
$$

Since the transfer function of the system and the output are already known, the desired input $U^{*}(s)$ can be derived using:

$$
U^{*}(s)=\frac{Y(s)}{H(s)}
$$

Substituting equations (4.9) and (4.11) into (4.12) and after some simplification, the desired input can be expressed as:

$$
U^{*}(s)=\frac{P_{M}\left(\tau_{b} s+1\right)\left(\tau_{c} s+1\right)}{c_{a} s\left(c_{b} \tau_{b} s+1\right)\left(c_{c} \tau_{c} s+1\right)}
$$

By equating (4.10) and (4.13) and matching both sides term by term, the 


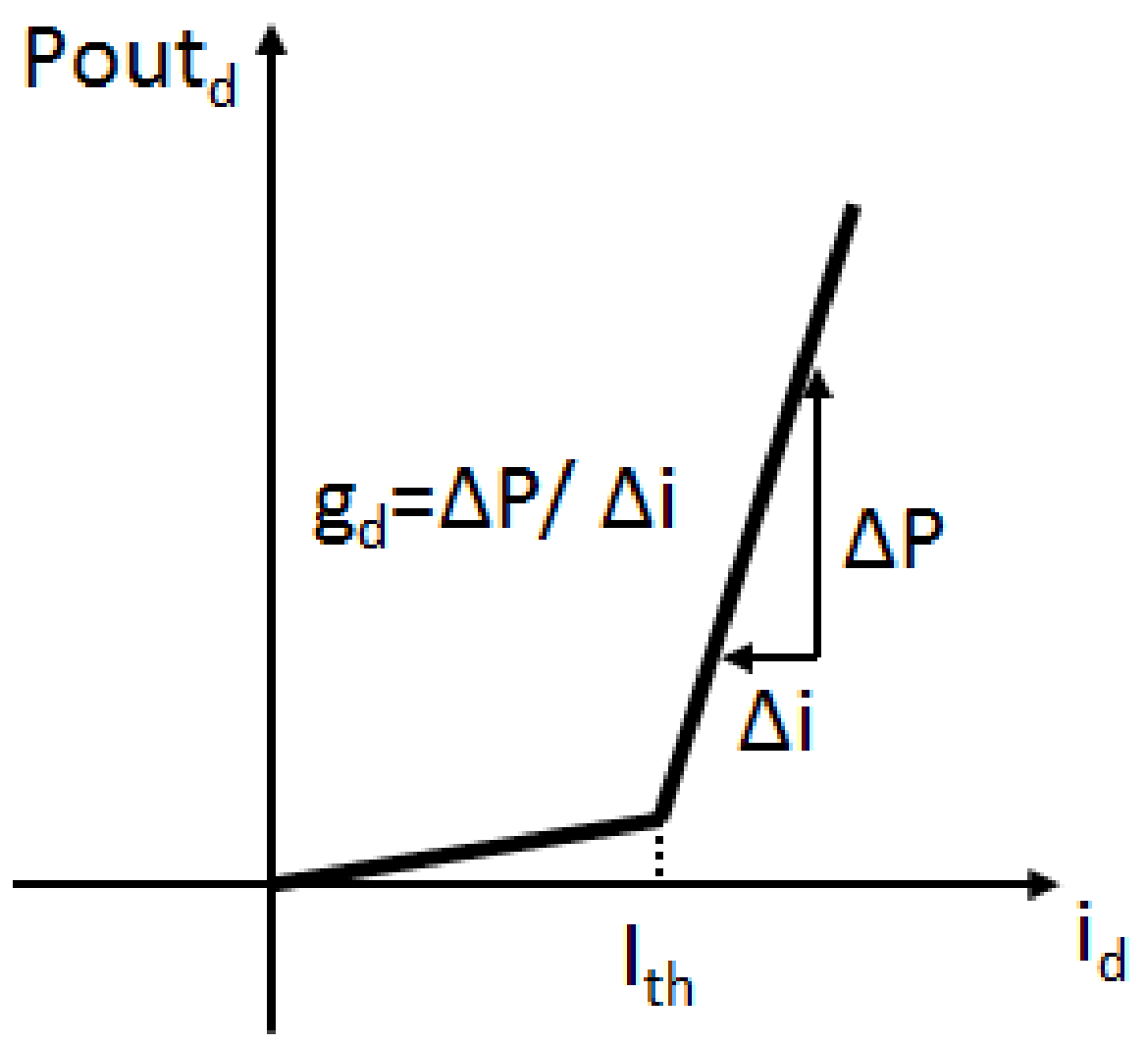

Figure 4.7: Relationship between input laser current and output laser power. Threshold current $I_{t h}$ and the gain $g_{d}$ are calibrated and known in advance.

parameters of optimal input laser power $U(s)$ can be derived as:

$$
\begin{aligned}
& P_{0}=\frac{P_{M}}{c_{a}}, \\
& P_{1}=\frac{\left(1-c_{b}\right)\left(c_{b} \tau_{b}-\tau_{c}\right)}{c_{b}\left(c_{b} \tau_{b}-c_{c} \tau_{c}\right)} P_{0}, \\
& P_{2}=\frac{\left(1-c_{c}\right)\left(c_{c} \tau_{c}-\tau_{b}\right)}{c_{c}\left(c_{c} \tau_{c}-c_{b} \tau_{b}\right)} P_{0}, \\
& \tau_{1}=c_{b} \tau_{b}, \\
& \tau_{2}=c_{c} \tau_{c} .
\end{aligned}
$$

In practice, the overshoot circuit controls the laser current instead of laser power. To complete the work, the laser power profile needs to be converted to laser current for actual implementation. The relationship between the input laser current 
and output laser power of a laser diode is shown in Figure 4.7. The parameters of the threshold current $I_{t h}$ and gain of operating range $g_{d}$ can be determined by the calibration process, which measures the laser output power by using a photodiode to detect the strength of the output laser light while sweeping the input laser current. These two parameters are known to us in advance and are not part of the HAMR writing process model. As shown, the laser diode generates very little laser output when the input laser current is less than $I_{t h}$. When the laser current is bigger than $I_{t h}$, the output laser power is proportional to the input laser current with gain of $g_{d}$. The final laser current can then be derived from the output laser power profile using:

$$
I_{\text {laser }}(t)=I_{t h}+\frac{P_{0}}{g_{d}}+\frac{P_{1}}{g_{d}} e^{-t / \tau_{1}}+\frac{P_{2}}{g_{d}} e^{-t / \tau_{2}} .
$$

To verify the effectiveness of the pole placement method discussed above, the optimized input laser power profile is calculated based on parameter values shown in Table 4.1 and equation (4.14). Such input profile is injected into the original non-linear model and the signal magnitude is observed at the model output. It is compared with other three different input profiles: no overshoot or compensation, baseline single overshoot input laser power profile, and the profile derived using the deconvolution method, which will be discussed in the next section. All the four input laser power profiles are plotted in Figure 4.8 for comparison. And the simulation results of the signal magnitude corresponding to each input profile are shown in Figure 4.9 .

\subsubsection{Laser Current Profile Design via Deconvolution Method}

In this section, the optimized laser current profile is derived by using the deconvolution method. The same system model shown in Figure 4.6 can be used to 
Table 4.1: Identified Parameters of Linearized Model

\begin{tabular}{|l|l|}
\hline \hline Symbol & Value Identified from Test Drive \\
\hline$c_{a}$ & $7.6 e-3$ \\
\hline$c_{b}$ & 0.75 \\
\hline$\tau_{b}$ & $0.168 \mu \mathrm{s}$ \\
\hline$c_{c}$ & 0.83 \\
\hline$\tau_{c}$ & $0.9 \mu \mathrm{s}$ \\
\hline
\end{tabular}

represent the writing process. Previous studies showed that this system is approximately linear and the deconvolution method [159] can be applied as a result. Let $u[n]$ be the input to the model and the model output can be derived using convolution in the discrete time domain as shown in equation (4.16), where $h[n]$ is the unit sample response of the system.

$$
y[n]=h[n] * u[n] .
$$

Now, the task is to find the unit sample response of the system $h[n]$. Firstly, a step input $u[n]$ is applied, which is active laser power $P_{A}[n]$ in this case, and the written-in signal magnitude $S_{T}[n]$ is measured. The media thermal power $P_{M}[n]$ can then be calculated using:

$$
P_{M}[n]=g^{-1}\left(S_{T}[n]\right) .
$$

The $P_{M}[n]$ will be the output $y[n]$ of the system. Finally, the system unit sample response $h[n]$ can be derived using the deconvolution method as:

$$
h[n]=\operatorname{deconv}(y[n], u[n]) .
$$

Next, let's define the desired output $y^{*}(n)$ as a step function for the ideal case 


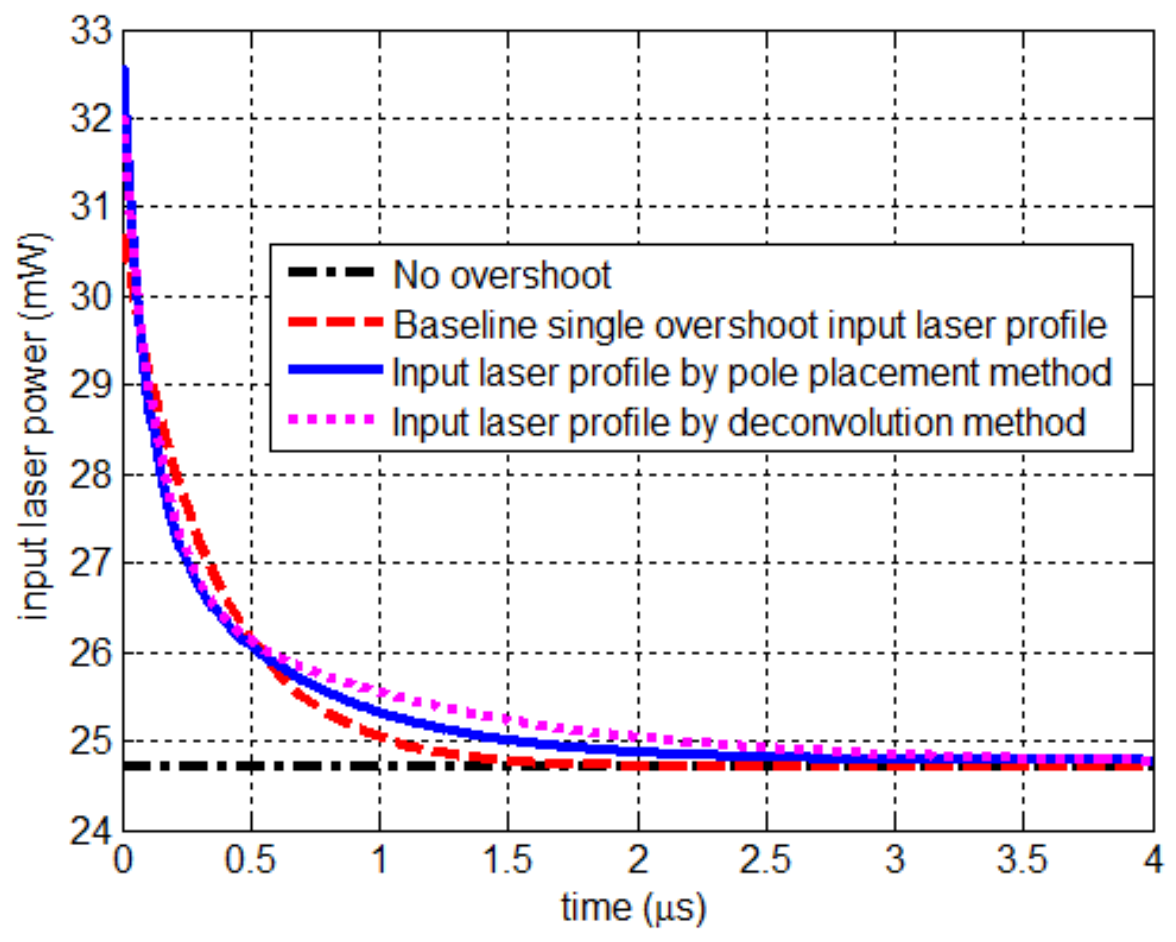

Figure 4.8: Comparison of different input laser power profiles. The two optimized profiles by pole placement and deconvolution method are able to produce approximately step change in signal magnitude at the model output. No overshoot and single overshoot input profiles are also included for comparison.

in order to minimize the impact of transient responses on signal magnitude. And the required input profile $u^{*}(n)$ can be derived using the deconvolution method as shown by equation (4.19) since the system unit sample response $h(n)$ is already known.

$$
u^{*}[n]=\operatorname{deconv}\left(y^{*}[n], h[n]\right) .
$$

In Figure 4.8, the comparison is made on the input laser power profiles identified from both the pole placement and the deconvolution methods. The results match each other quite well. There are other two profiles, no overshoot and single overshoot profiles, which are included as well for comparison.

All the four input laser profiles are applied to the original non-linear model and the signal magnitude of the model output is plotted in Figure 4.9. From the 


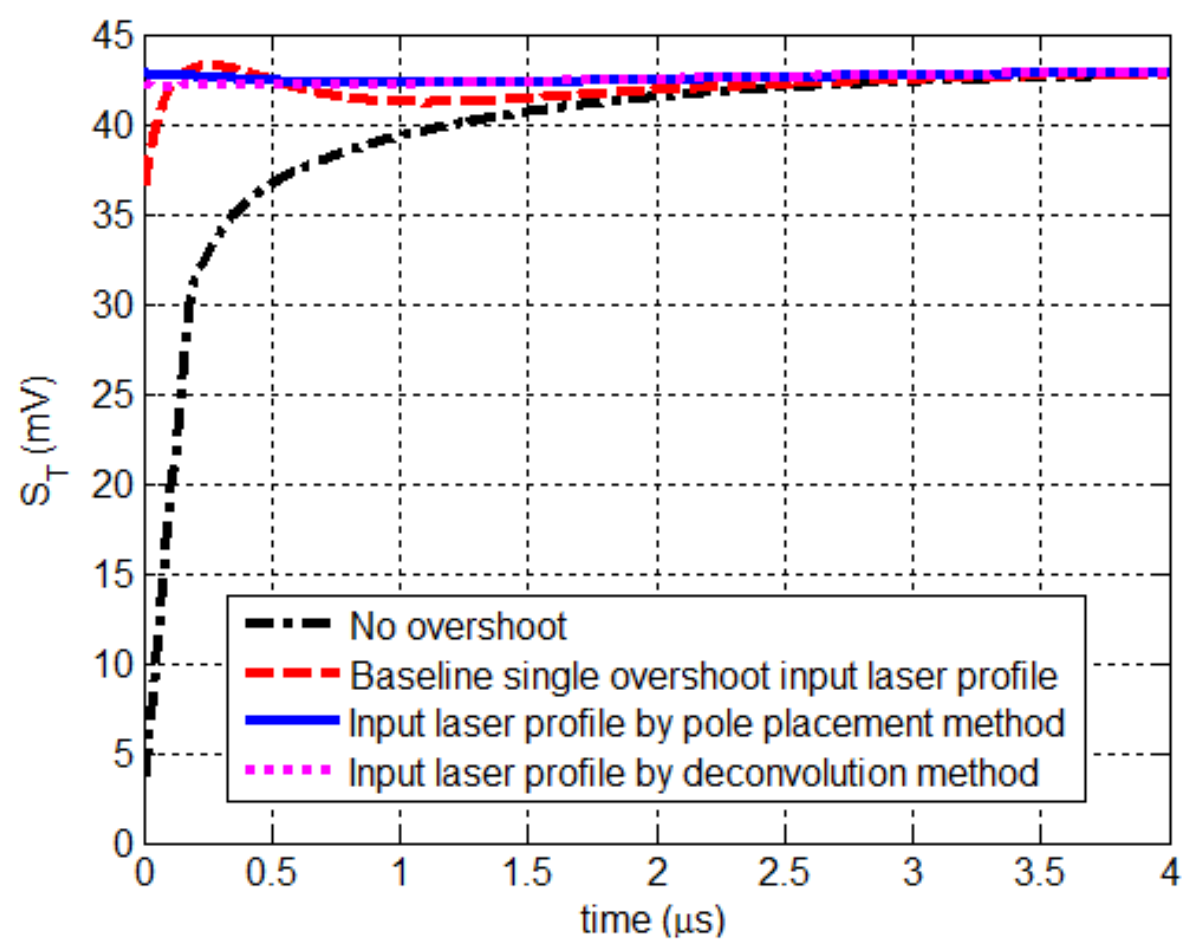

Figure 4.9: Signal magnitude transient response comparisons for different input laser profiles: no overshoot applied, only one overshoot exponential decay component applied and input laser profiles derived by both pole place and deconvolution methods.

figure, a few observations can be made:

- The output signal magnitude response takes more than $1 \mu s$ to reach steady state if no overshoot or compensation is applied.

- Using single overshoot compensation, the transient response cannot be completely eliminated.

- Using input laser profiles derived from both pole placement and deconvolution methods, the transient response can be reduced to a very small amount.

Although both the pole placement and deconvolution methods give very similar results, there are pros and cons for each of the methods.

The pros: 
- Pole placement method

- It gives a systematic way to derive the optimized input laser current profile.

- It shows that two overshoot compensation components are required, which can be used for pre-amplifier overshoot circuit design.

- Deconvolution method

- This method provides an easy and effective way to compensate for the transient impacts, especially in the drive calibration process.

- HAMR writing process modeling is not required.

- This method can generate any kind of input current profiles, which are not limited by the exponential function.

The cons:

- Pole placement method

- The model construction and identification process is complex.

- Only exponential decay overshoot component can be used.

- Deconvolution method

- This method does not provide deep insight of the transients involved in the writing process.

- The generated input current profile may not be easily implemented by the hardware circuit in pre-amplifier. 


\subsection{Conclusion}

The additional laser related heat sources in HAMR cause various protrusions which will change the critical NMS. In this chapter, it is first demonstrated on how to calculate the slow and NFT protrusions under three different writing conditions, namely RRO field write, servo pattern write and user data field write. Since the NFT protrusion can be estimated through the model, the NMS can be maintained close to its target value during writing process by shaping the input laser current and TFC input power, which can be solved as standard control system design problems. It is desired to minimize the NMS in order to increase the NFT efficiency. By doing to, the input laser power and the NFT temperature can be reduced to increase the lifetime of the NFT, which is one of the major bottlenecks for HAMR technology today [58]. However, the risk of NFT hitting media will increase as well when NMS is reduced. As a result, trade off must be made between NFT efficiency and the safe clearance of NFT and media. Finally, the details are presented for the compensation of NMS change under different writing duty cycles by TFC.

In the remainder of the chapter, two methods are shown to compensate for the impacts of fast transients, which include NFT protrusion and heat transient, so that the signal quality is good and consistent from the very beginning of the writing process until the end. The consistent media thermal profile ensures the good signal quality, minimum format efficiency loss (which happens when the compensation is not enough), and minimum adjacent track write (which happens when the compensation is too big). 


\section{Chapter 5}

\section{Burst Pattern Non-linearity}

\section{Improvement}

\subsection{Introduction}

In previous chapters, the HAMR writing model are constructed and identified. The model is helpful for writing good quality servo patterns by controlling the input laser current profiles and head fly-height. Now, the focus can be moved onto the improvement of burst pattern non-linearity in order to minimize the PES demodulation error and improve the track-following performance. Burst pattern written in the servo sector is used to generate the off-track PES signal, which is used as the feedback signal for head positioning control. Research work [32,33,161-166] summarized many kinds of burst patterns. Among them, ABCD and null burst patterns are the most commonly used. The ABCD burst pattern has four fields named as A, B, C and D respectively. Nowadays, most of the hard disk drives are using null burst pattern [167] since it has only two fields named as N and Q. The null burst pattern reduces the length of the burst pattern to a half compared with 


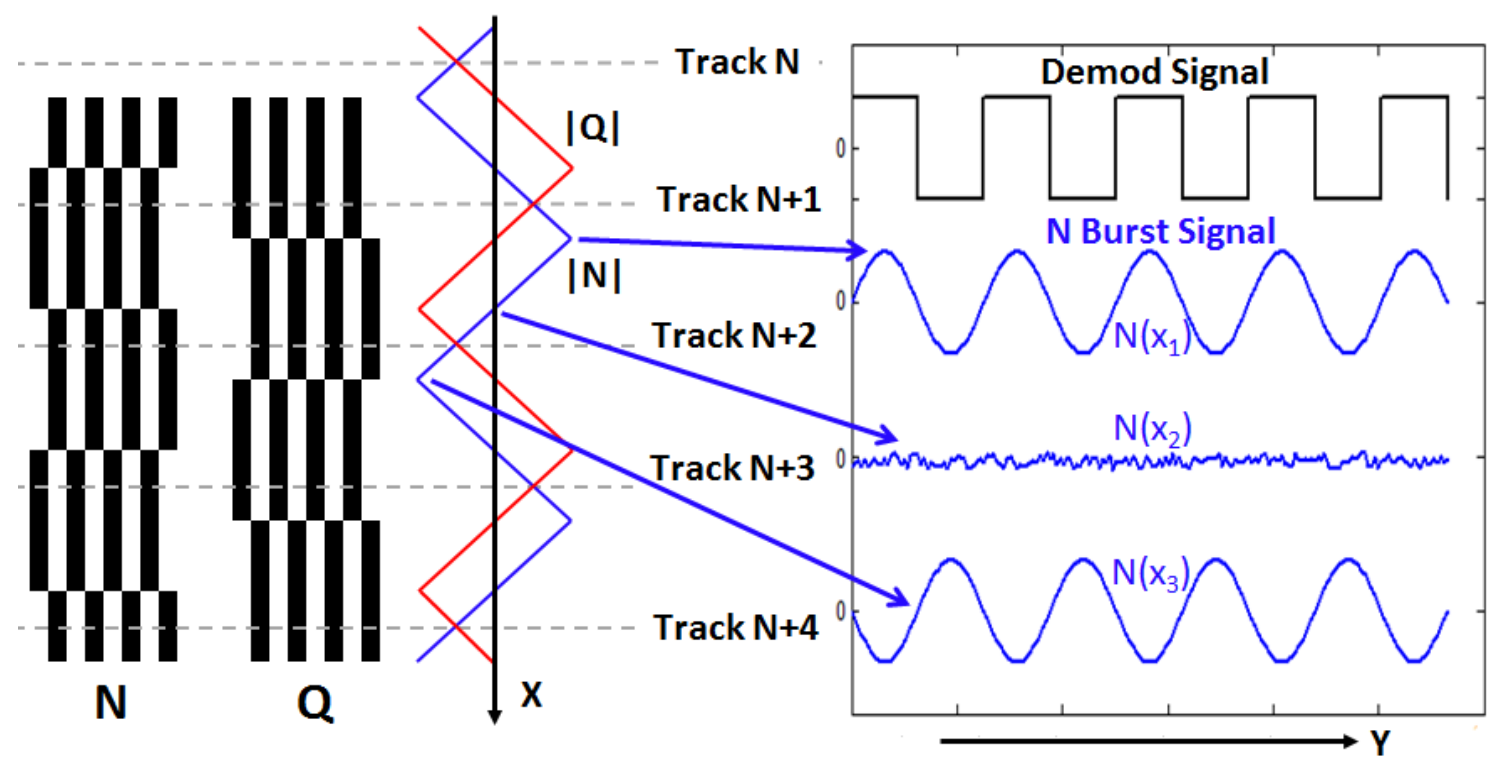

Figure 5.1: Illustration of Null phase burst demodulation [32].

ABCD burst and thus improves the overall format efficiency (FE) such that more space can be used for user data storage. However, in exchange for the FE gain, null burst pattern sacrifices the signal-to-noise ratio (SNR), suffer higher demodulation complexity and is subject to the phase jittering etc.

The detailed null burst pattern is shown in Figure 5.1 and the $\mathrm{N}$ and Q fields are shifted by a half-track in cross-track $(\mathrm{X})$ direction. For each of the field, the pattern is stitched for one track width with 180 degree phase shift in down-track (Y) direction. The demodulation is done by integration of the product of demodulation signal and the burst signal [32]. Three special reader cross-track locations are picked up, where the integration values are maximum positive, 0 and maximum negative respectively as shown in Figure 5.1. The PES signal is derived from burst signal N and Q based on (5.1). Equation (5.1) clearly shows that the PES demodulation is non-linear in nature.

$$
P E S(x)=\frac{|Q(x)|-|N(x)|}{\max (|N(x)|,|Q(x)|)} .
$$

as shown in [168] 


\subsection{PES Non-Linearity Characterization}

Equation (5.1) clearly shows that the PES demodulation is non-linear. In addition, burst pattern could be distorted by many factors such as the non-linearity of the detection circuits, non-uniform of the written-in servo pattern, skew angle, erase band $[169,170]$, servo writing process and spindle speed and so on. Since the PES demodulation is non-linear, people are trying to characterize and correct it $[168,171-175]$. Cooper et al. PES non-linearity was analyzed by using third order polynomial to model the non-linearity of the detection circuit and magnetoresistive (MR) head respectively based on the ABCD burst pattern [171]. The non-linearity caused by distortion of PES saturation and the on-track dead band is analyzed in [176]. Patent by Nazarian et al. [172] shows a method to measure the burst nonlinearity and construct the correction values. Patent by Asgari et al. [173] invents a new method to construct PES and maintain the continuity, which is further improved in [168] for both ABCD and null burst patterns.

The characteristics of the burst profiles are mainly determined by their shapes. However, it is quite difficult to describe an irregular shape fully and accurately. As a result, only two parameters, flatness [25] and asymmetry, will be used to quantify the burst shape, which are believed to be the most important. The burst flatness is defined in (5.2), where $B_{\text {crs }}$ is the burst magnitude where $\mathrm{N}$ and Q burst across each other and $B_{\max }$ is the maximum magnitude of the $\mathrm{N}$ or $\mathrm{Q}$ burst as illustrated in Figure 5.2. Please take note that the $\mathrm{N}$ and $\mathrm{Q}$ crossing point is defined as the track center or $0 \%$ off-track location.

$$
\text { Flatness }=\frac{B_{c r s}}{B_{\max }} \text {. }
$$

as defined in $[25]$ 


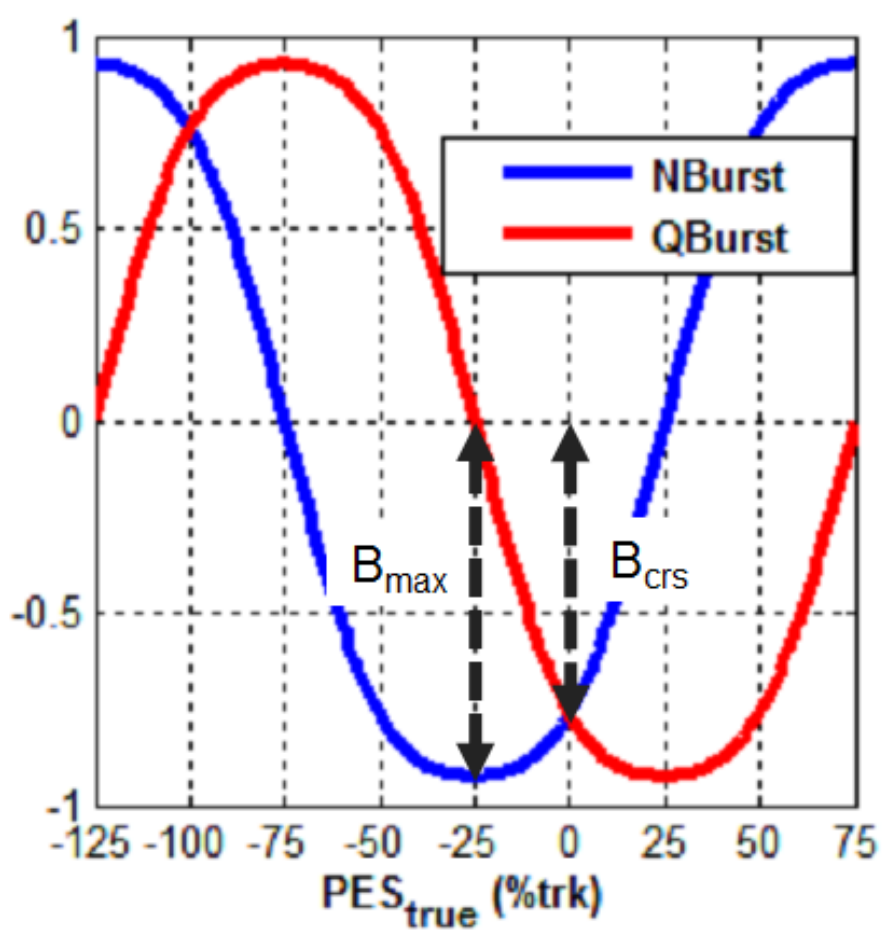

Figure 5.2: Concept of burst flatness.

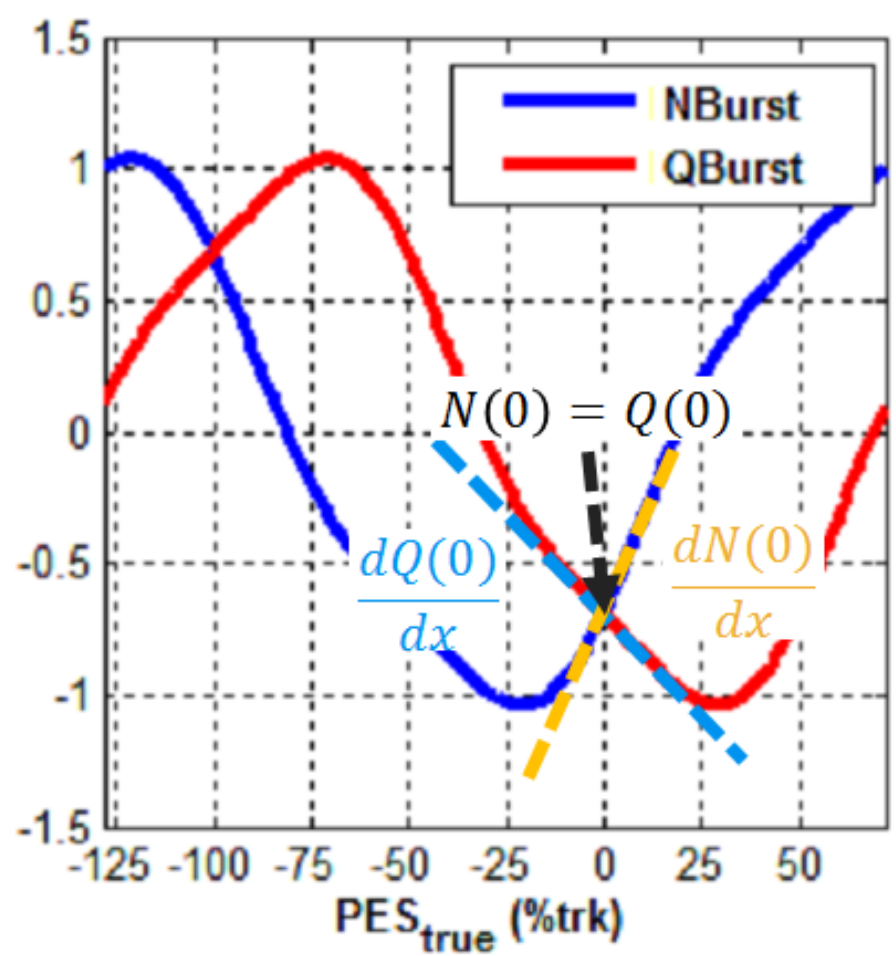

Figure 5.3: Concept of burst asymmetry. 
In addition, together with former colleague Hong Fan, we define burst asymmetry as (5.3) with reference to [177]. As shown in Figure 5.3, $\frac{d N(0)}{d x}$ and $\frac{d Q(0)}{d x}$ are the slope of the $\mathrm{N}$ and $\mathrm{Q}$ bursts at their crossing point respectively.

$$
A s m y=\left(\frac{d N(0)}{d x}+\frac{d Q(0)}{d x}\right) /(2 N(0))
$$

\subsection{Ideal Burst Pattern}

In this section, the efforts have been made to find out the best possible shape of burst pattern, which will result in the minimum PES non-linearity through simulation. The focus is only on the null burst pattern since it is the most commonly used pattern in today's HDDs. It is assumed that both of $\mathrm{N}$ and $\mathrm{Q}$ bursts have the same shape and repeat itself for every two tracks. The cross-track phase shift between $\mathrm{N}$ and Q bursts is half of a track. Since N and Q bursts are the periodic functions of off-track location, they can be decomposed by Fourier series into different harmonic components. The $1 X$ harmonic will be treated as the baseline burst shape.

Let the baseline ideal $\mathrm{N}$ burst to be the waveform defined as a cosine function of the period of 2 tracks. In our model, the baseline $\mathrm{Q}$ burst is constructed from the baseline $\mathrm{N}$ burst by simply shifting it to the right by half of a track. Then, the PES can be derived by using (5.1). The ideal PES, which will be denoted as PES ${ }_{i}$, should be a straight line with the slope of 1. However, as shown in Figure 5.4, the PES derived from baseline $\mathrm{N}$ and Q bursts is not a straight line, which will be called raw PES and denoted as $P E S_{r}$ in the rest of this chapter. The difference between $P E S_{r}$ and $P E S_{i}$ is called PES correction values or $P E S_{c}$ as shown in the bottom 

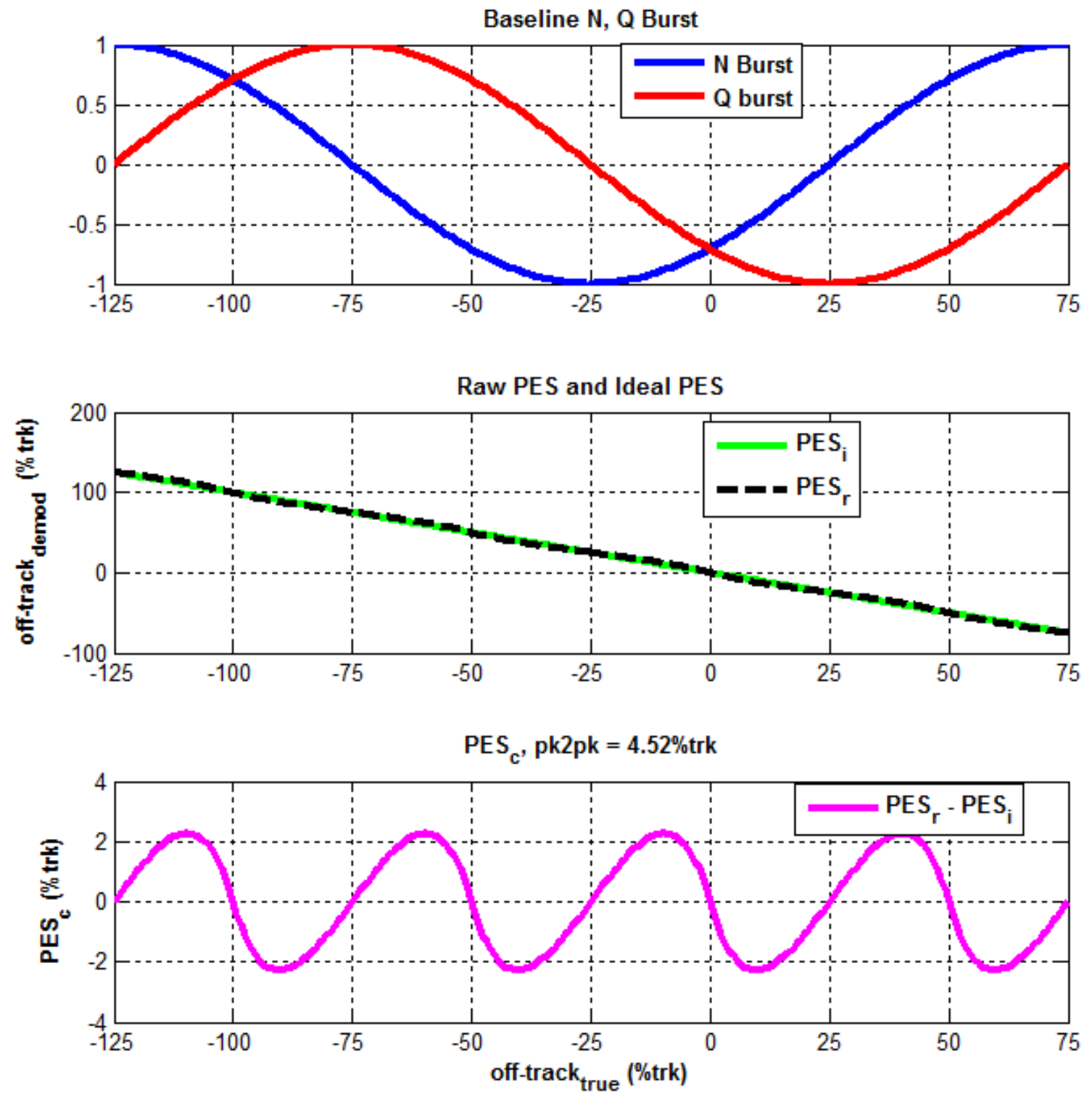

Figure 5.4: Demodulation and non-linearity of baseline Null burst pattern.

of Figure 5.4. For the baseline bursts, the peak-to-peak value of $P E S_{c}$ is $4.52 \% \mathrm{trk}$.

$$
P E S_{c}(x)=P E S_{r}(x)-P E S_{i}(x) .
$$

Next, it is tried to change the shape of burst by adding different harmonics to the baseline burst according to (5.5). The $1 X$ sine harmonic is set to 0 since it will not alter the shape of the baseline burst except changing the burst magnitude and 
phase only. The magnitude of the burst is normalized and the phase shift between $\mathrm{N}$ and $\mathrm{Q}$ burst is fixed at half of a track.

$$
N_{\text {burst }}(x)=c\left(\cos (\pi x)+\sum_{n=2}^{\infty}\left(a_{n} \cos (n \pi x)+b_{n} \sin (n \pi x)\right)\right) .
$$

The harmonics from 2 to $5 X$ are studied with amplitudes of $+/-10 \%$ of the baseline burst and all other higher order harmonics are ignored since their magnitudes tend to be very small. Each harmonic is studied individually and the distorted $\mathrm{N}$ burst is the sum of baseline burst and each individual distortion harmonic. The distorted Q burst is constructed by simply shifting the distorted $\mathrm{N}$ burst by half of a track. And the track center is defined as the cross point of two burst profiles. The distortion effect of each harmonic are studied by focusing on the following aspects:

- The shape of the distorted burst.

- The period of PES error and its peak-to-peak value.

- DC squeeze which happens when the distance between the two track centers does not equal to one track.

The detailed plots for all the distorted burst profiles are shown in Appendix A and the distortion effects are summarized in Table 5.1. 
Table 5.1: Identification Results

\begin{tabular}{|c|c|c|c|c|c|c|c|c|c|c|c|c|c|c|c|c|}
\hline & $\begin{array}{c}\cos \\
+3 \mathrm{X}\end{array}$ & $\begin{array}{c}\cos \\
-3 \mathrm{X}\end{array}$ & $\begin{array}{c}\cos \\
+5 \mathrm{X}\end{array}$ & $\begin{array}{c}\cos \\
-5 \mathrm{X}\end{array}$ & $\begin{array}{c}\sin \\
+3 \mathrm{X}\end{array}$ & $\begin{array}{c}\sin \\
-3 \mathrm{X}\end{array}$ & $\begin{array}{c}\sin \\
+5 \mathrm{X}\end{array}$ & $\begin{array}{c}\sin \\
-5 \mathrm{X}\end{array}$ & $\begin{array}{c}\cos \\
+2 \mathrm{X}\end{array}$ & $\begin{array}{c}\cos \\
-2 \mathrm{X}\end{array}$ & $\begin{array}{c}\cos \\
+4 \mathrm{X}\end{array}$ & $\begin{array}{c}\cos \\
-4 \mathrm{X}\end{array}$ & $\begin{array}{c}\sin \\
+2 \mathrm{X}\end{array}$ & $\begin{array}{c}\sin \\
-2 \mathrm{X}\end{array}$ & $\begin{array}{c}\sin \\
+4 \mathrm{X}\end{array}$ & $\begin{array}{c}\sin \\
-4 \mathrm{X}\end{array}$ \\
\hline $\begin{array}{c}\text { Burst Symmet- } \\
\text { ric about Peak }\end{array}$ & $\sqrt{ }$ & $\sqrt{ }$ & $\sqrt{ }$ & $\sqrt{ }$ & $X$ & $X$ & $X$ & $X$ & $X$ & $X$ & $X$ & $X$ & $X$ & $X$ & $X$ & $X$ \\
\hline $\begin{array}{c}\text { Burst 1-track } \\
\text { Periodic }\end{array}$ & $\sqrt{ }$ & $\sqrt{ }$ & $\sqrt{ }$ & $\sqrt{ }$ & $\sqrt{ }$ & $\sqrt{ }$ & $\sqrt{ }$ & $\sqrt{ }$ & $X$ & $X$ & $X$ & $X$ & $X$ & $X$ & $X$ & $X$ \\
\hline $\begin{array}{c}\text { PES Error Half- } \\
\text { track Periodic }\end{array}$ & $\sqrt{ }$ & $\sqrt{ }$ & $\sqrt{ }$ & $\sqrt{ }$ & $\sqrt{ }$ & $\sqrt{ }$ & $\sqrt{ }$ & $\sqrt{ }$ & $X$ & $X$ & $X$ & $X$ & $X$ & $X$ & $X$ & $X$ \\
\hline $\begin{array}{c}\text { PES Error 1- } \\
\text { track Periodic }\end{array}$ & $\sqrt{ }$ & $\sqrt{ }$ & $\sqrt{ }$ & $\sqrt{ }$ & $\sqrt{ }$ & $\sqrt{ }$ & $\sqrt{ }$ & $\sqrt{ }$ & $X$ & $X$ & $X$ & $X$ & $X$ & $X$ & $X$ & $X$ \\
\hline $\begin{array}{c}\text { Improve Basel- } \\
\text { ine PES Error }\end{array}$ & $X$ & $\sqrt{ }$ & $\sqrt{ }$ & $X$ & $X$ & $X$ & $X$ & $X$ & $X$ & $X$ & $X$ & $X$ & $X$ & $X$ & $X$ & $X$ \\
\hline $\begin{array}{c}\text { No DC } \\
\text { Squeeze }\end{array}$ & $\sqrt{ }$ & $\sqrt{ }$ & $\sqrt{ }$ & $\sqrt{ }$ & $\sqrt{ }$ & $\sqrt{ }$ & $\sqrt{ }$ & $\sqrt{ }$ & $\sqrt{ }$ & $\sqrt{ }$ & $X$ & $X$ & $\sqrt{ }$ & $\sqrt{ }$ & $\sqrt{ }$ & $\sqrt{ }$ \\
\hline
\end{tabular}



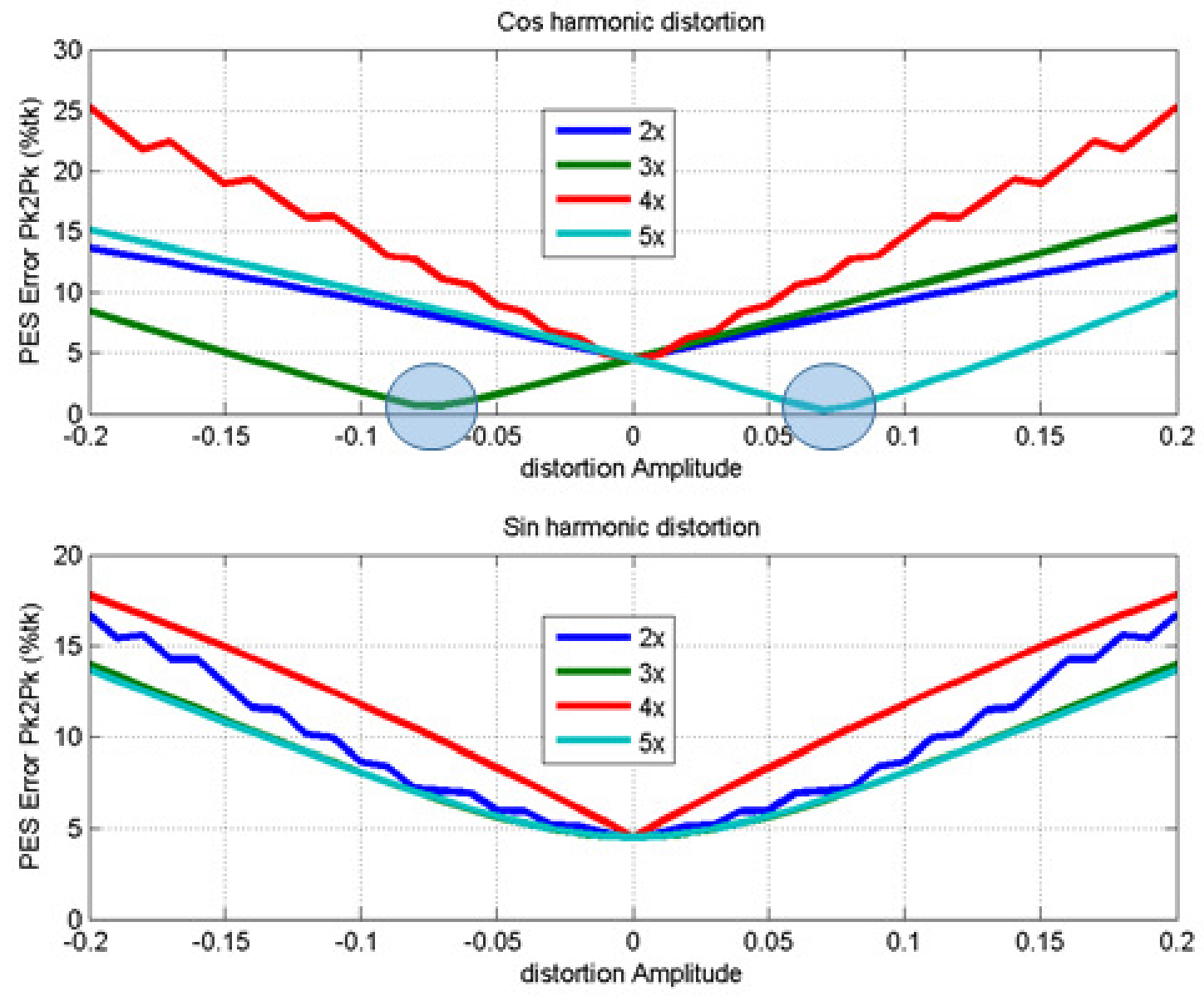

Figure 5.5: PES error vs. distortion amplitude.

From the above results, the following conclusions can be drawn:

- $P E S_{c}$ is periodic in half-track for odd harmonic distortion, which means that the correction table can be only half-track wide [173]. However, $P E S_{c}$ has the period of 2 tracks wide for even harmonic distortion and need a bigger correction table to do the correction.

- Some of the even harmonic such as $4 X$ cosine harmonic will cause DC squeeze, which can only be corrected by two-tracks wide table.

- $-3 X$ cosine and $+5 X$ cosine harmonics of small amplitude will improve the baseline $P E S_{c}$, and all other harmonics will make the baseline $P E S_{c}$ worse. 

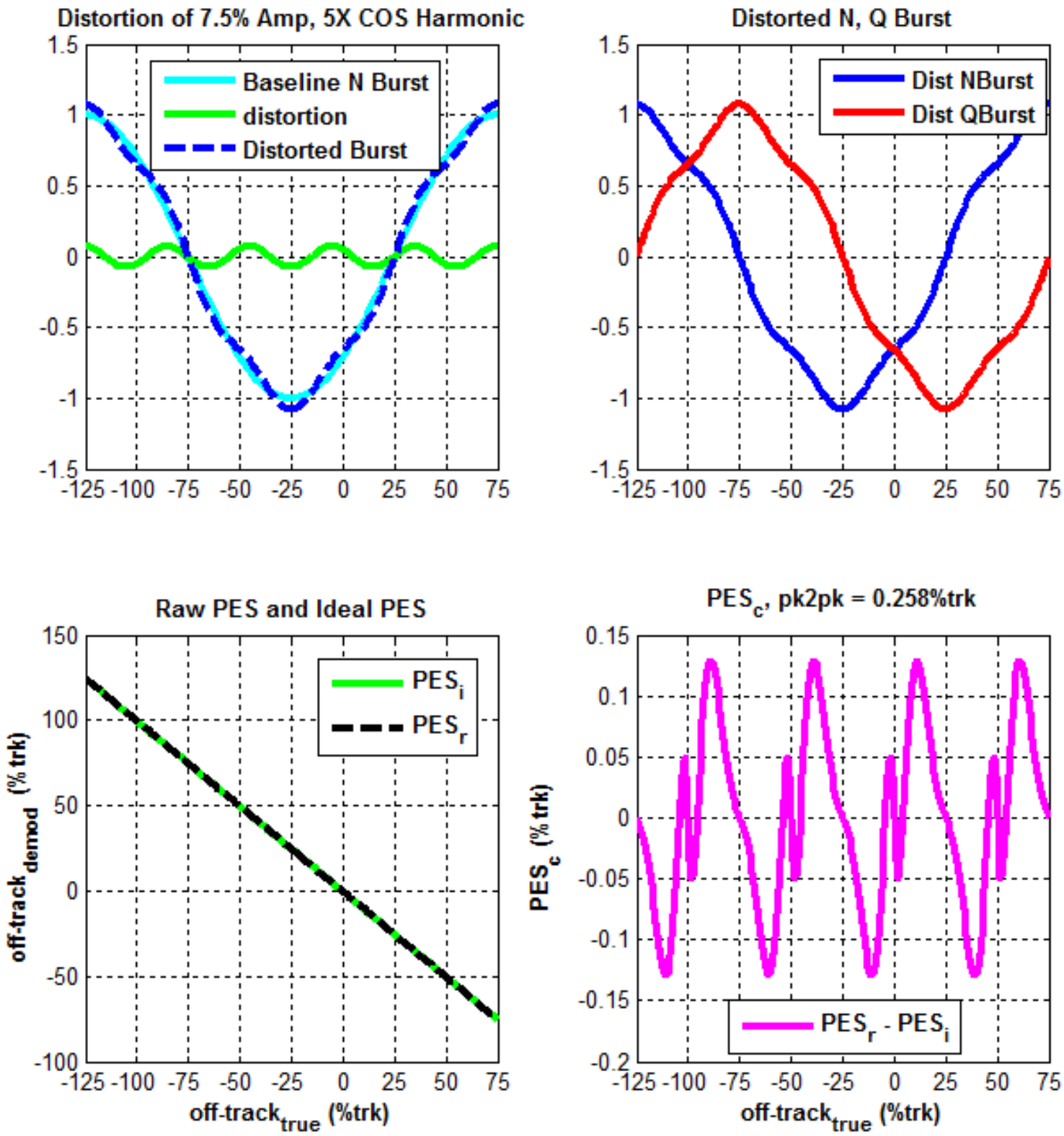

Figure 5.6: Burst with 7.5\% 5X cos harmonic distortion.

To further verify the impact of the amplitude of each harmonic on $P E S_{c}$, the harmonic amplitude are swept from $-20 \%$ to $20 \%$, and $P E S_{c}$ are calculated for each case. The results are shown in Figure 5.5. It is confirmed that only $3 X$ and $5 X$ cosine harmonic can reduce $P E S_{c}$. And the minimum $P E S_{c}$ can be achieved by harmonic amplitude around $-7.5 \%$ and $7.5 \%$ respectively. For $7.5 \% 5 X$ cosine harmonic distortion, the peak-to-peak value of $P E S_{c}$ can be reduced to $0.258 \%$ trk 

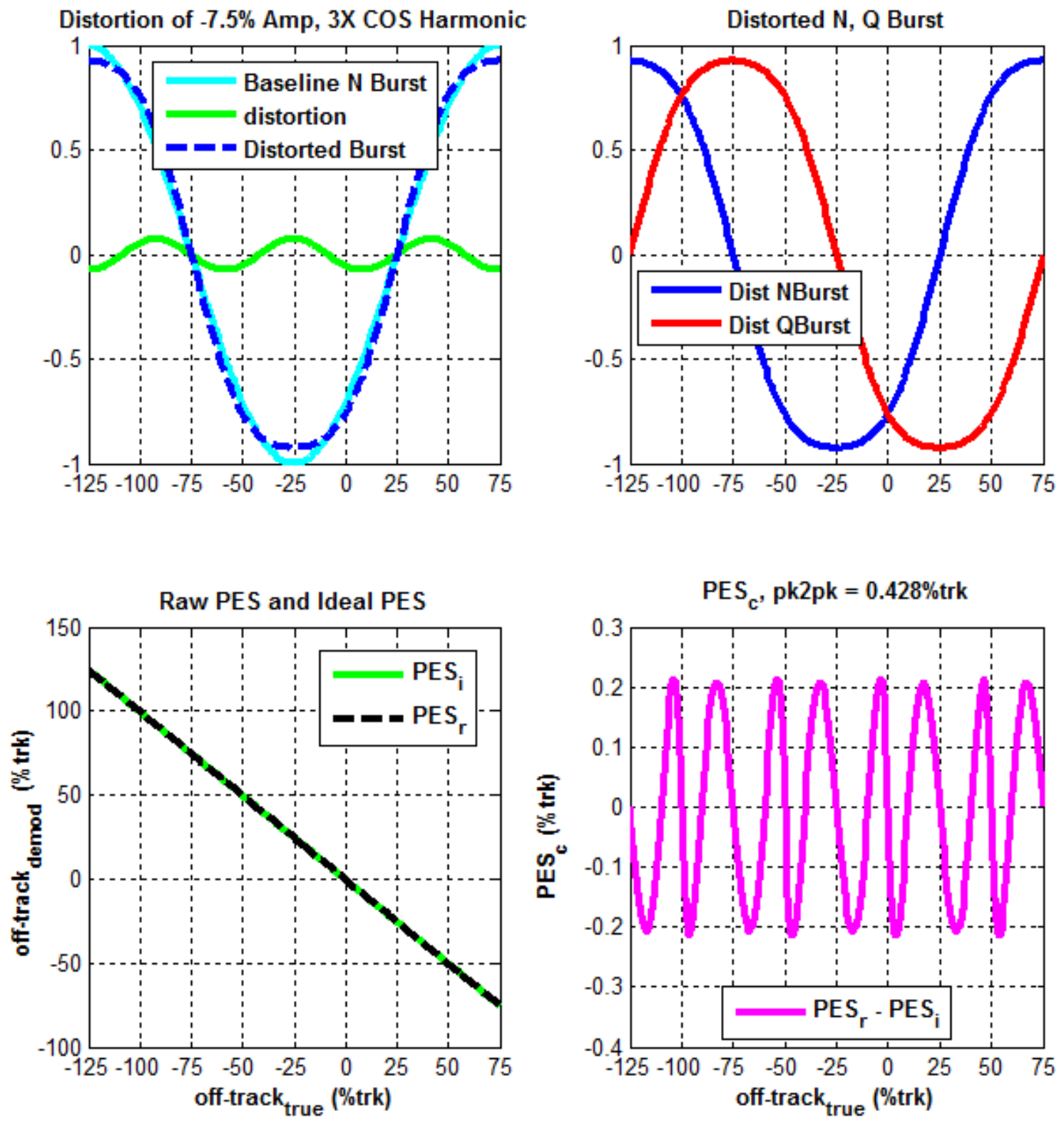

Figure 5.7: Burst with $-7.5 \% 3 X \cos$ harmonic distortion.

as shown in Figure 5.6. However, in the real drive, this kind of burst shape does not exist because the real burst profile has flattened top and bottom due to head media properties such as the fringing field effect, the geometric shape of the head etc $[171,178]$. Instead, the flat top burst shape resulted from $-7.5 \% 3 X$ cosine harmonic distortion is desired and practical. The resulted peak-to-peak value of $P E S_{c}$ is also very small at $0.428 \%$ trk as shown in Figure 5.7. From this study, it can be concluded that the best burst shape should be symmetric with flatness 


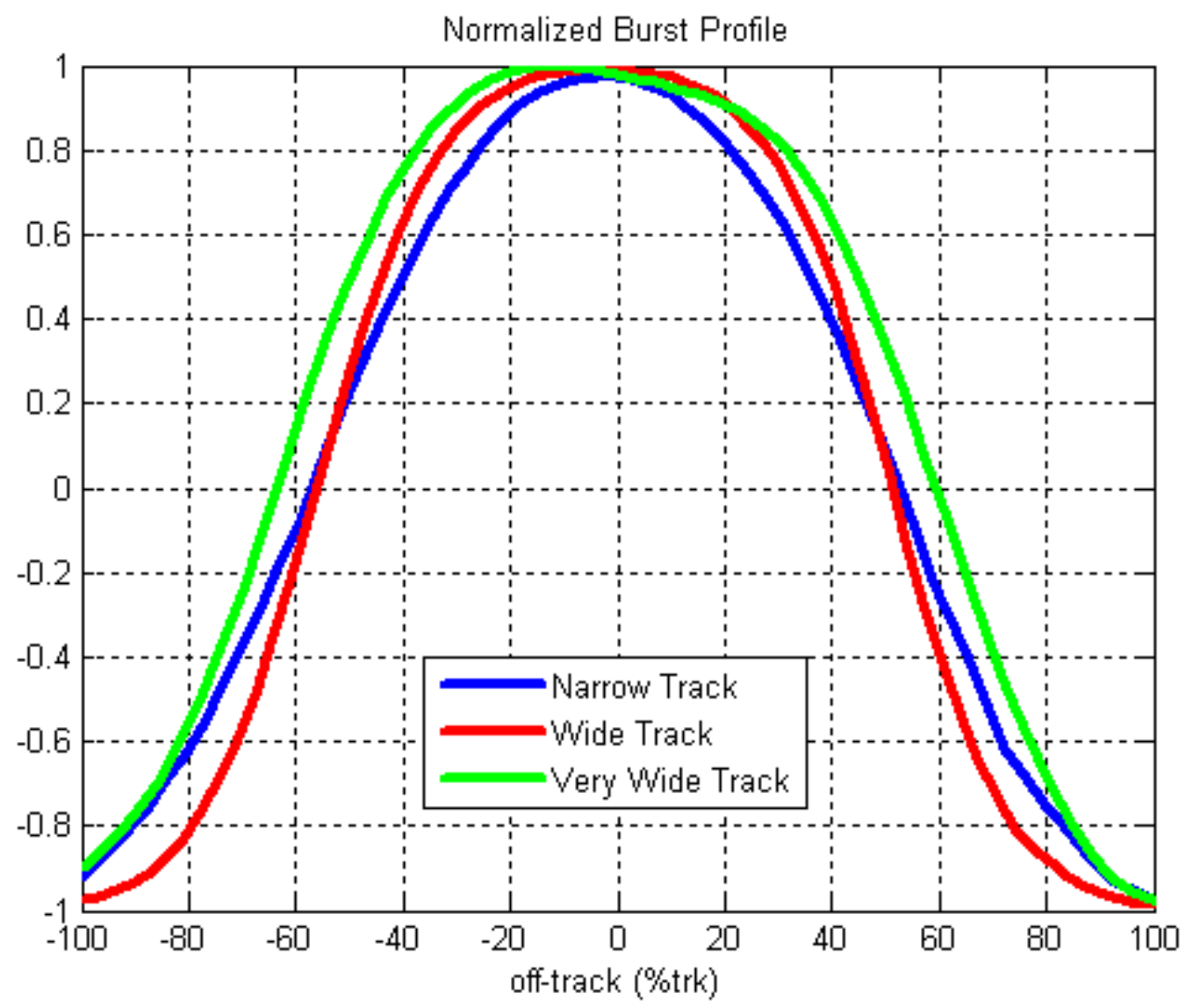

Figure 5.8: Burst profile comparison for different track width.

around 0.82 from (5.2).

\subsection{Improve Written-in Burst Pattern}

\section{Non-linearity}

From previous studies, the desired burst profile should have flatness around 0.82 and the asymmetry should be minimized as much as possible. Some research works have been focusing on the effects of reader width $[179,180]$ and erase band [181] on the non-linearity of the PES signal. The reader width is normally defined as the width of the micro-track profile at its $50 \%$ amplitude. In the extreme case, if the track pitch is too big compared to the reader width, the burst profile will look more 
like a trapezoid shape, which generally will have higher flatness. In contrast, if the track is too narrow, the burst profile will look more like a triangle. The burst asymmetry is also related to the track width. The wide track will tend to produce asymmetric burst profiles. The detail is illustrated in Figure 5.8. In tradition HDDs, the servo track pitch is fixed for all the drives build with the same model of the head. However, due to the variation of the production process, the reader width is not a constant but varies according to some distributions. If the requirement on the burst linearity is not very high, this approach is acceptable. However, when the areal density is increased to a few $T b / \mathrm{in}^{2}$, it is desired to improve the burst linearity as much as possible to improve the track-following performance. This research proposal is to optimize the servo track pitch for each individual head such that the peak-topeak value of $P E S_{c}$ can be minimized for all the heads.

An experiment was carried out on a particular head at different location of the disk, namely the outer diameter (OD), the middle diameter (MD) and the inner diameter (ID). Null burst patterns are written with different track pitch and $P E S_{c}$ are measured thereafter. The $P E S_{c}$ can be decomposed by the Fourier series using Equation (5.6). Since $B_{1}$ coefficient is the biggest contributor, $B_{1}$ coefficient is plotted against the ratio of reader width to track width for all the three locations. As shown in Figure 5.9, the minimum $P E S_{c}$ is achieved when the ratio between reader width and track pitch is around $68 \%$ for this particular head, where $B_{1}=0$. The similar method can then be used to find out the optimized servo track pitch during the drive calibration process.

$$
P E S_{c}(x)=\sum_{k} A_{k} \cos (k \omega x)+B_{k} \sin (k \omega x) .
$$




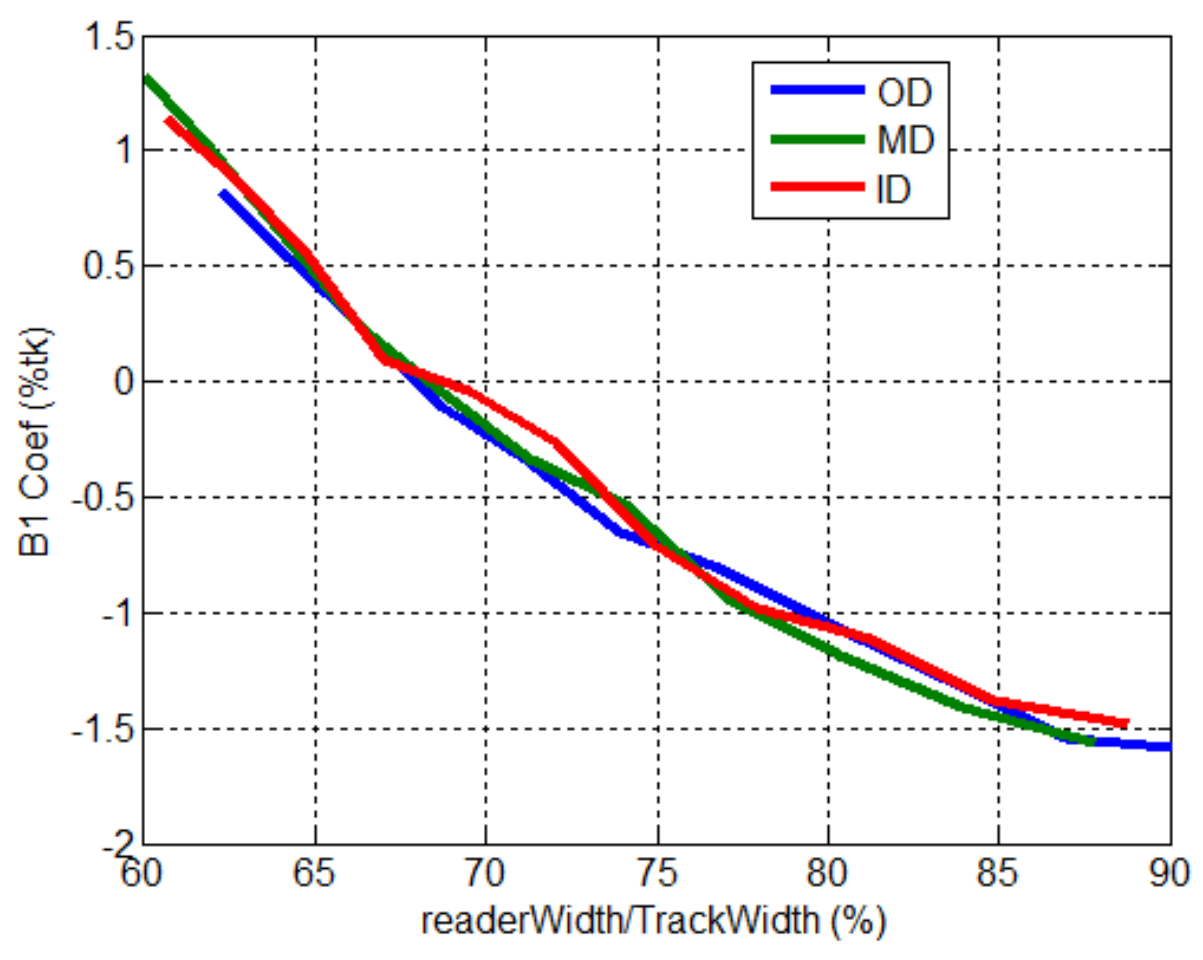

Figure 5.9: $B_{1}$ coefficient of Fourier series for $P E S_{c}$ versus the ratio of reader to track width.

\subsection{Conclusion}

In this chapter, the burst non-linearity and its relationship with respect to the shape of the null phase burst profile are discussed. The ideal burst profile shape is identified, which produces minimum non-linearity by using simulations. The burst shape can be manipulated by controlling the ratio between the reader width and the servo track pitch. And the burst non-linearity can be improved with better burst shape. The benefits of the minimizing the burst non-linearity are:

- Minimize the effort required for burst correction and reduce the burst correction residue error.

- Better burst linearity will improve the linear interpolation accuracy of the RRO compensation values used for CWRRO scheme which will be discussed in the next chapter. 


\section{Chapter 6}

\section{Continuous Wedge Repeatable Run-out Compensation}

\subsection{Introduction}

Previous chapter focuses on the minimization of the burst non-linearity in order to improve the PES demodulation accuracy. Smaller demodulation error will improve the track-following performance in "average" sense since it applies to all the servo wedges. In contrast, there are random noises and disturbances, which make accurate track-following a very challenging task with the increasing areal density of HDDs. These disturbances can be classified into two categories, non-repeatable run-out (NRRO) and repeatable run-out (RRO). The RRO is mainly due to the disk eccentricities, the imperfection of spindle motor assembly, the disturbance and noise during servo writing process, and the randomness of the magnetic grains. The RRO is normally cancelled in two ways. The first one is to "follow" the RRO [35-39]. This method works best for the frequency components, which are coherent from track to track. This kind of RRO is normally in the low-frequency range, 

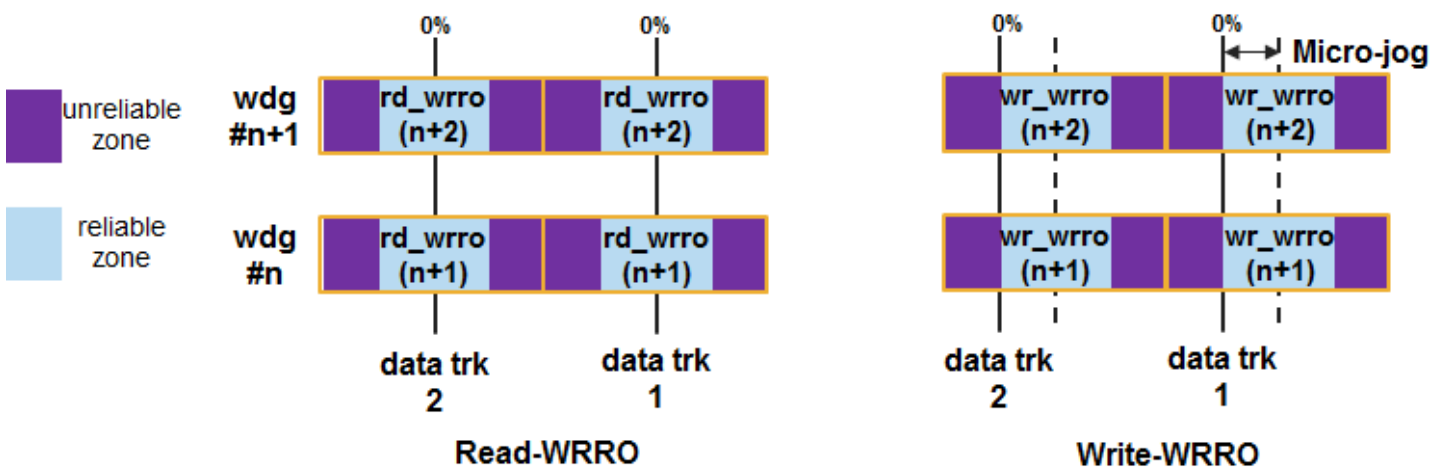

Figure 6.1: Illustration of read-WRRO and write-WRRO.

and the RRO is learned in real-time, which is also known as online learning. The second method is to "anti-follow" the RRO [40-46,182]. This method works best for non-coherent frequency components, which may cause over-writing to the adjacent tracks as explained in Chapter 2. Using this method, the learned RRO values are subtracted from the PES so that the head trajectory will follow the ideal circular track. Both of these two methods are implemented to tackle different frequency components. And sometimes, they can even be applied to the same frequency component.

This thesis only focuses on the "anti-follow" method. The RRO mentioned thereafter all refers to non-coherent RRO unless otherwise specified. In current modern HDDs, RRO values are learned offline for each data track and stored in the servo sector, and is called wedge RRO (WRRO). Since the reader and writer are separated by some distance, there is an offset between the reader and writer center in the radial direction, which is called micro-jog. The micro-jog amount varies for the different skew angle. The micro-jog is 0 for 0 skew angles at middle diameter (MD) location and reaches the maximum value at extreme outer diameter (OD) and inner diameter (ID). Due to the existence of micro-jog, the RRO compensation values are different for the read and write operations and need to be learned and stored separately, which are called read-WRRO and write-WRRO respectively. The basic idea is shown in Figure 6.1. 
The traditional WRRO (TWRRO) compensation scheme mentioned above is working perfectly for the conventional magnetic recording. However, when the data track pitch becomes smaller and smaller due to increased areal density, the TWRRO scheme will not work properly due to the reduction in off-track read capability (OTRC). For example, using SMR technology, the gap between two data tracks does not exist any more [21]. Furthermore, the data track width is designed to be a little bit wider than the reader only. Since the WRRO fields have the same width as the data track, the WRRO OTRC is substantially reduced. If under vibration condition, the WRRO value may not be decoded. In the worse case, the wrong values could be applied such that the track-following performance will be badly degraded.

To overcome the above-mentioned issues, a new WRRO scheme, continuous WRRO (CWRRO) is proposed. The major differences between CWRRO and TWRRO are:

- Each CWRRO field will occupy two tracks and zig-zag between adjacent two RRO fields.

- CWRRO is learned and stored based on servo track instead of data track. There is no need to differentiate read-WRRO and write-WRRO as in TWRRO any more.

- For CWRRO, since the data track center can be located at any offset location of the servo track, the RRO must be learned at multiple offset locations per servo track and use them to estimate RRO at any other off-track location. The trade-off between the number of learning locations, the format efficiency and the total learning time must be made.

In the remaining of the chapter, the multi-track blending (MTB) technique will be introduced, which improves the TMR performance of the basic CWRRO 


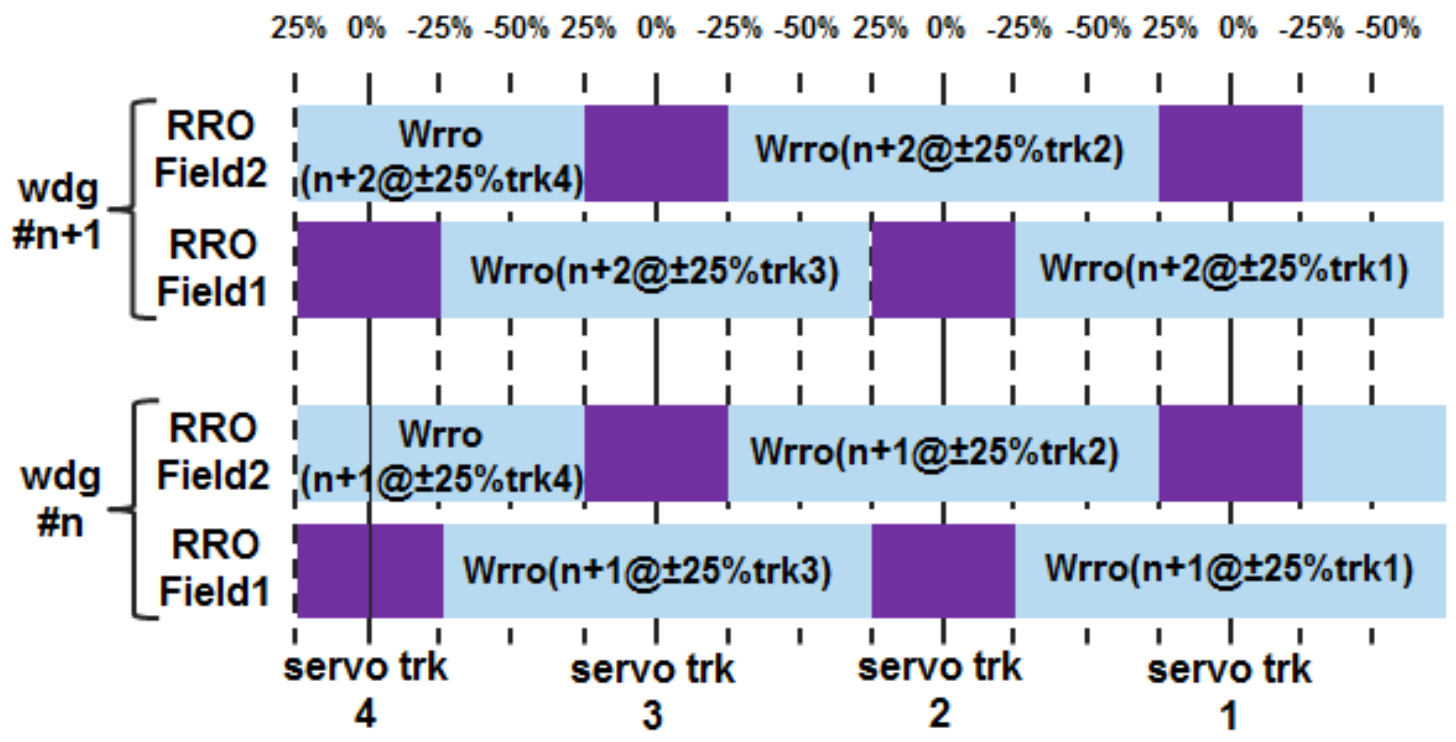

Figure 6.2: Illustration of CWRRO.

scheme without incurring any format efficiency loss.

\subsection{Multi-Track Blending for CWRRO}

In this section, the layout between TWRRO and CWRRO are compared first in Figures 6.1 and 6.2. Figure 6.1 shows the layout for TWRRO, where the readWRRO is shown on the left and the write-WRRO is on the right. The light blue and purple zone are where the WRRO values can be read back reliably and unreliably respectively. The width of these two zones is determined by OTRC. TWRRO is associated with the data track. Read-WRRO is written at the data track center and write-WRRO is written to the location whose distance to data track center is equal to the micro-jog amount. Please take note that micro-jog can be more than one track wide. Figure 6.2 illustrates the layout for the proposed CWRRO. The RRO values are learned and stored with respect to the servo tracks. There are two RRO fields, RRO field 1 and RRO field 2, in each wedge and they are written in a zig-zag manner as shown in Figure 6.2. Each field occupies two tracks including 
unreliable zone in order to improve the OTRC. The RRO field 1 stores two values learned for odd tracks at $+/-25 \%$ off-track location, and RRO field 2 stores values for the even tracks. When the data read/write operation is performed, the read position could be at any offset locations. And the RRO compensation value at that location can be estimated by linear interpolation of two stored RRO values which are learned nearby $[26,27]$. The proposed layout ensures that the required RRO values are always available. One of the drawbacks of CWRRO is that it occupies double space compared to TWRRO with both read-WRRO and write-WRRO, or four times compared to TWRRO with only write-WRRO. Another drawback is the performance degradation if the head location falls in between the learning locations. And it will be elaborated more in the following.

When the head location is in between the nearby two learning locations, the WRRO compensation value at that location $C_{x}$ is estimated by linear interpolation as shown in Figure 6.3. However, in order for this kind of estimation to be meaningful, the true WRRO values at the current head position should be dependent on the WRRO values at learning locations in some degree. The correlation coefficient can be used to measure their dependency. The definition of the correlation coefficient for two vectors is shown in (6.1), where $\mathrm{X}$ and $\mathrm{Y}$ are vectors containing WRRO values learned at each location. The $\operatorname{cov}()$ in the equation is the covariance operator. Firstly, WRRO are learned at $+/-25 \%$ off-track location for multiple tracks respectively, and then cascaded into one vector as $L_{-25 \%}$ and $L_{25 \%}$ respectively. Next, WRRO are learned at locations in between $+/-25 \%$ off-track location and stored into vectors in a similar manner. Finally, the correlation coefficients of all the learning locations with respective to $L_{-25 \%}$ and $L_{25 \%}$ are calculated. The results are shown in Figure 6.4. As shown, the correlation coefficient is always bigger than 0 but decreases when the distance between their learning locations increases. This implies that the estimation error will increase when the head location is further away from the learning locations. 


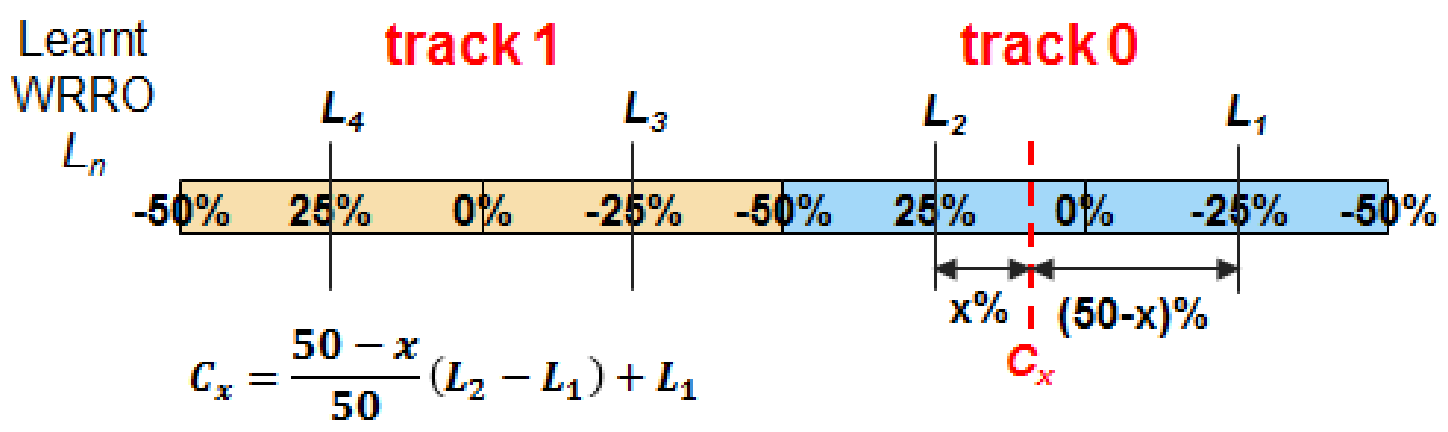

Figure 6.3: Concept of linear interpolation.

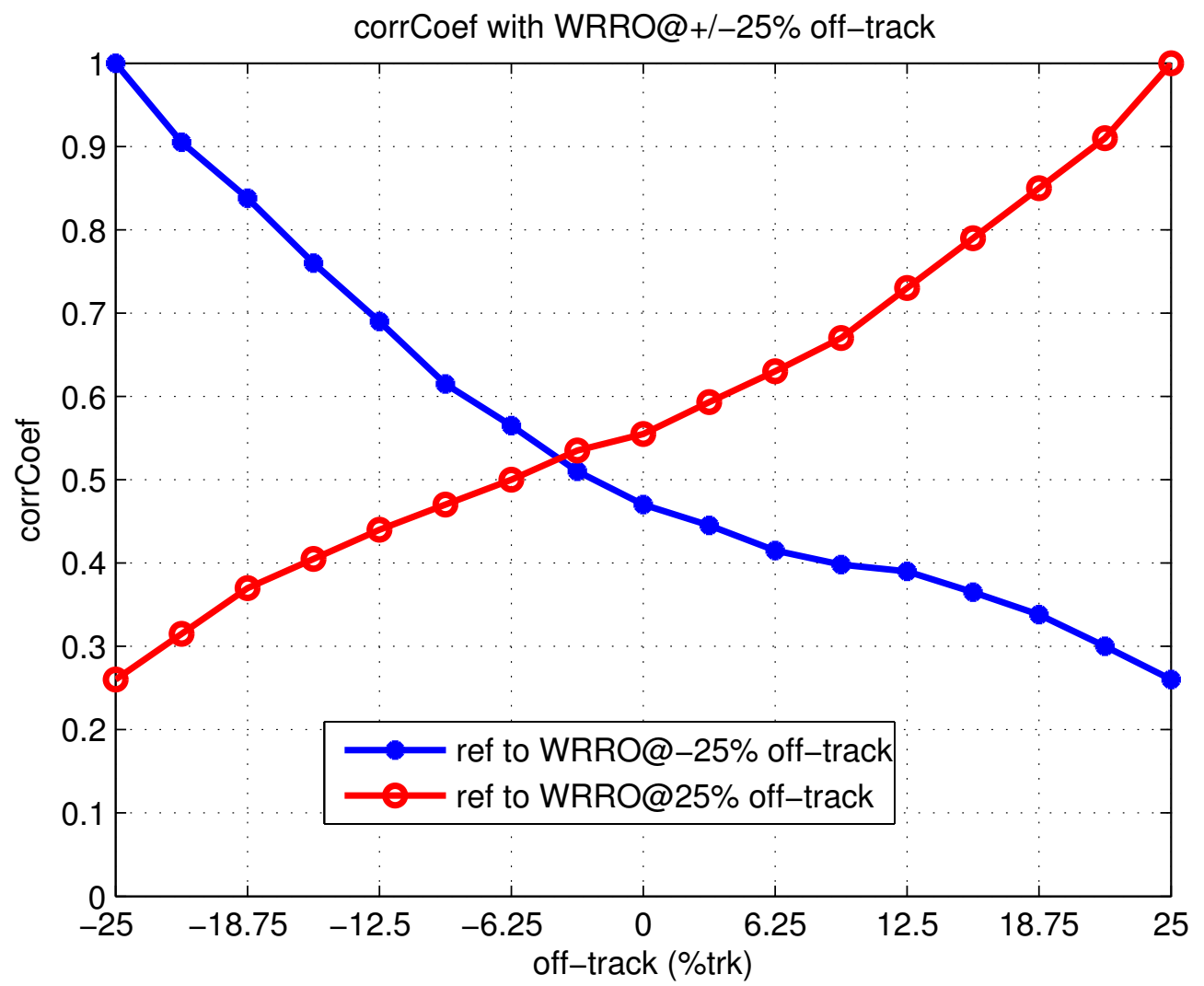

Figure 6.4: Correlation coefficient of WRRO at different distance.

$$
\rho_{X Y}=\frac{\operatorname{cov}(X, Y)}{\sigma_{X} \sigma_{Y}}
$$

In Figure 6.5, the TMR performance is compared for TWRRO and the basic scheme of CWRRO. TWRRO has the best performance at the learning location and 


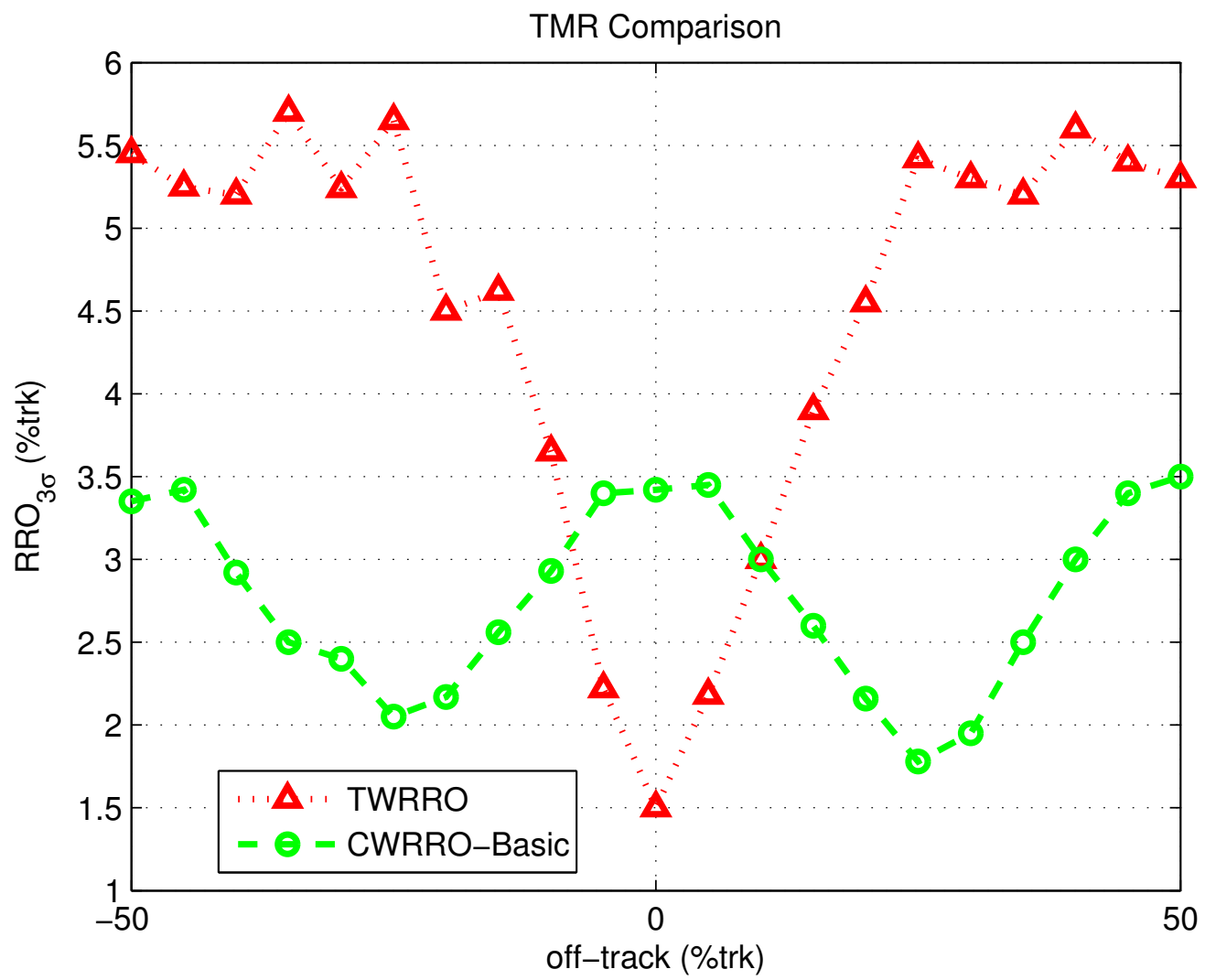

Figure 6.5: TMR performance comparison between TWRRO and basic CWRRO scheme.

degrades quickly when the head moves away. CWRRO also have lowest TMR at learning locations of $+/-25 \%$ off-track and the worst TMR happens at the center in between the learning locations. As shown, the worst CWRRO TMR is bigger than $10 \%$ off-track TMR for TWRRO. Since our maximum off-track target is $10 \%$ of the track pitch, the basic scheme CWRRO cannot meet the specification. In order to improve the CWRRO performance, the most effective way is to reduce the distance between learning locations such as four locations per servo track instead of two. However, if all the values are stored in the servo sector, it will occupy twice of the space than the basic CWRRO scheme, which cannot meet the format efficiency requirement.

The multi-track blending is a trade-off between TMR performance and the 


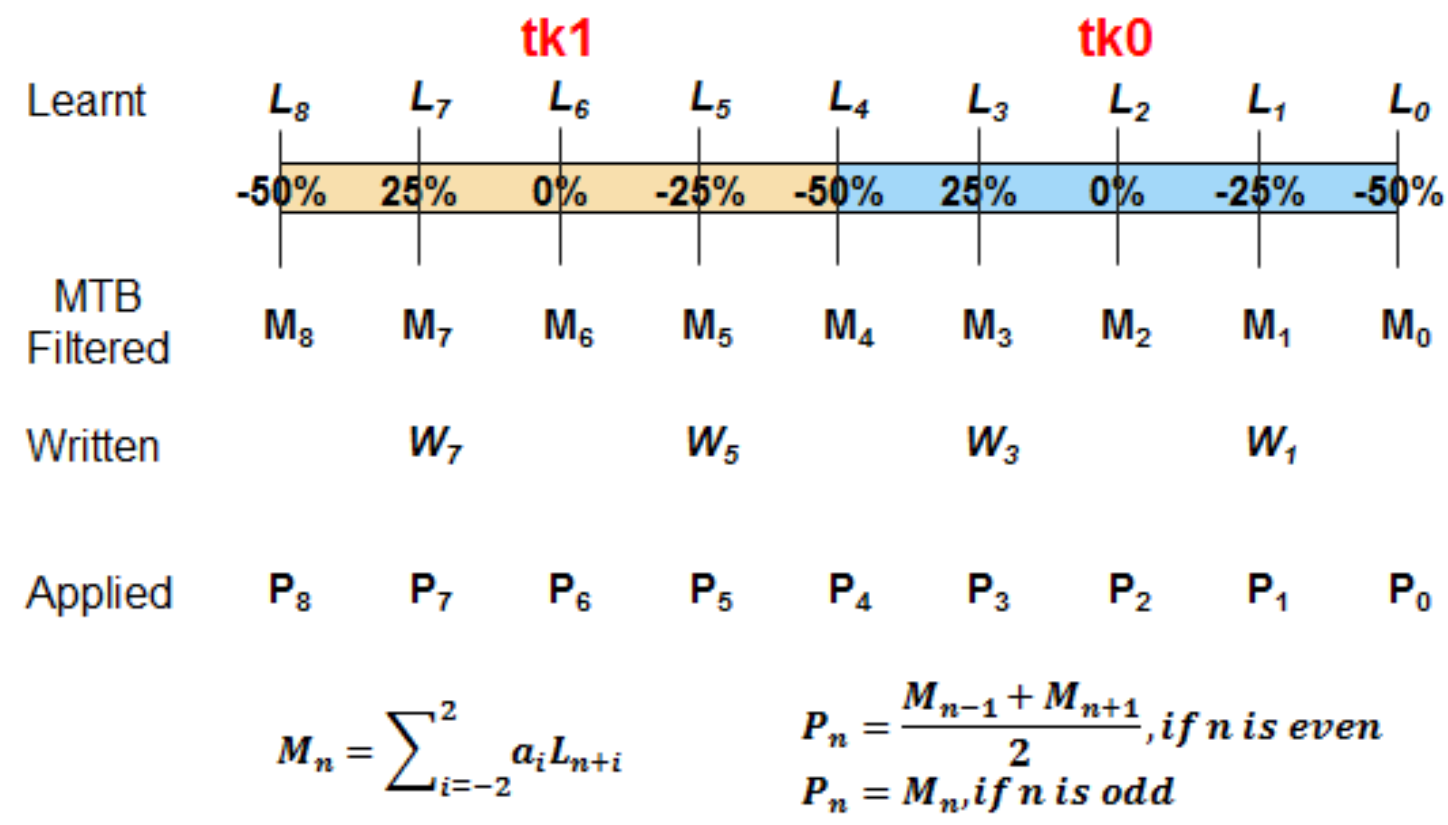

Figure 6.6: Concept of MTB.

format efficiency. The basic idea of MTB is illustrated in Figure 6.6. The WRRO values are learned for four locations per track at $-50 /-25 / 0 / 25 \%$ off-track respectively and are stored in the vectors denoted as $L_{n}$ for all the wedges. For each wedge, the learned WRRO values are filtered by MTB filter and the output is called $M_{n}$, where $a_{i}$ are the filter coefficients. Next, $M_{n}$ is down-sampled by 2 to get $W_{n}$, which will be stored in the servo sectors. As a result, it has the same format efficiency as basic CWRRO scheme and the interpolation distance is still $50 \%$ of track pitch. In order to find the MTB filter coefficients, the mean square error (MSE) between $L_{n}$ and $P_{n}$ is minimized, where $P_{n}$ is derived based on (6.2). The MSE is defined in (6.3).

$$
\begin{gathered}
P_{n}=\left\{\begin{array}{l}
\frac{M_{n-1}+M_{n+1}}{2}, \text { if } n \text { is even, } \\
M_{n}, \text { if } n \text { is odd. }
\end{array}\right. \\
M S E=\sum_{k=1}^{n} E\left\{\left(P_{k}-L_{k}\right)^{2}\right\} .
\end{gathered}
$$


Let the order of MTB filter to be $2 N+1$, then the output of filter is defined as:

$$
M_{k}=\sum_{i=-N}^{N} a_{i} L_{k+i}, a_{i} \text { is filter coefficients. }
$$

Substituting (6.2) and (6.4) into (6.3) leads to:

$$
\begin{aligned}
M S E & =\sum_{k=1,3,5 \cdots}\left(E\left\{\left(P_{k}-L_{k}\right)^{2}\right\}+E\left\{\left(P_{k+1}-L_{k+1}\right)^{2}\right\}\right) \\
& =\sum_{k}\left(E\left\{\left(M_{k}-L_{k}\right)^{2}\right\}+E\left\{\left(\frac{M_{k}+M_{k+2}}{2}-L_{k+1}\right)^{2}\right\}\right) \\
& =\sum_{k}\left(E\left\{\left(\sum_{i=-N}^{N} a_{i} L_{k+i}-L_{k}\right)^{2}\right\}\right. \\
& \left.+E\left\{\left(\sum_{i=-N}^{N} \frac{a_{i}}{2}\left(L_{k+i}+L_{k+2+i}\right)-L_{k+1}\right)^{2}\right\}\right)
\end{aligned}
$$

Find the partial derivative with respect to each filter coefficient and set it to 0 :

$$
\begin{aligned}
\frac{\partial M S E}{\partial a_{i}} & =\sum_{k=1,3,5 \cdots} E\left\{2\left(\sum_{i=-N}^{N} a_{i} L_{k+i}-L_{k}\right) L_{k+j}\right\} \\
& +E\left\{\left(\sum_{i=-N}^{N} \frac{a_{i}}{2}\left(L_{k+i}+L_{k+2+i}\right)-L_{k+1}\right)\left(L_{k+j}+L_{k+2+j}\right)\right\} \\
& =0, j \in[-N, N] .
\end{aligned}
$$


With some simplification:

$$
\begin{aligned}
\sum_{i=-N}^{N}\left(\sum_{k=1,3,5 \cdots}(\right. & 5 E\left\{L_{k+i} L_{k+j}\right\}+E\left\{L_{k+i} L_{k+2+j}\right\} \\
& \left.\left.+E\left\{L_{k+2+i} L_{k+j}\right\}+E\left\{L_{k+2+i} L_{k+2+j}\right\}\right)\right) a_{i} \\
=\sum_{k=1,3,5 \cdots}\left(2 E\left\{L_{k+1} L_{k+2+j}\right\}\right. & \\
& \left.+4 E\left\{L_{k} L_{k+j}\right\}+2 E\left\{L_{k+1} L_{k+j}\right\}\right), j i n[-N, N] .
\end{aligned}
$$

This can be written in matrix form as:

$$
\begin{gathered}
A x=b . \\
x=A^{-1} b .
\end{gathered}
$$

Equation (6.9) can be used to find all the filter coefficients, which are contained in vector $x$. Next, the order of the MTB filters are going to be figured out. Firstly, the order is required to be odd as shown in the previous derivation. Next, since the reader width is normally less than $80 \%$ of the track pitch, 5 th order $(N=2)$ should be enough since the area covered by $L_{n-2}$ to $L_{n+2}$ is $100 \%$ of the track pitch. To verify our choice, a 7 th order filter is designed. However, the higher order filter coefficients are almost 0 . This proves that 5 th order is adequate. After the filter order is determined, the optimized filter coefficients are identified at OD, MD, and ID of the disk respectively. As shown in Figure 6.7, the coefficients are symmetric which indicate that the reader micro-track profile of this particular head is symmetric. In the meantime, the coefficients are very similar for all the three zones. This fact makes the implementation of MTB very simple since only five coefficients can be used for the whole disk surface. 


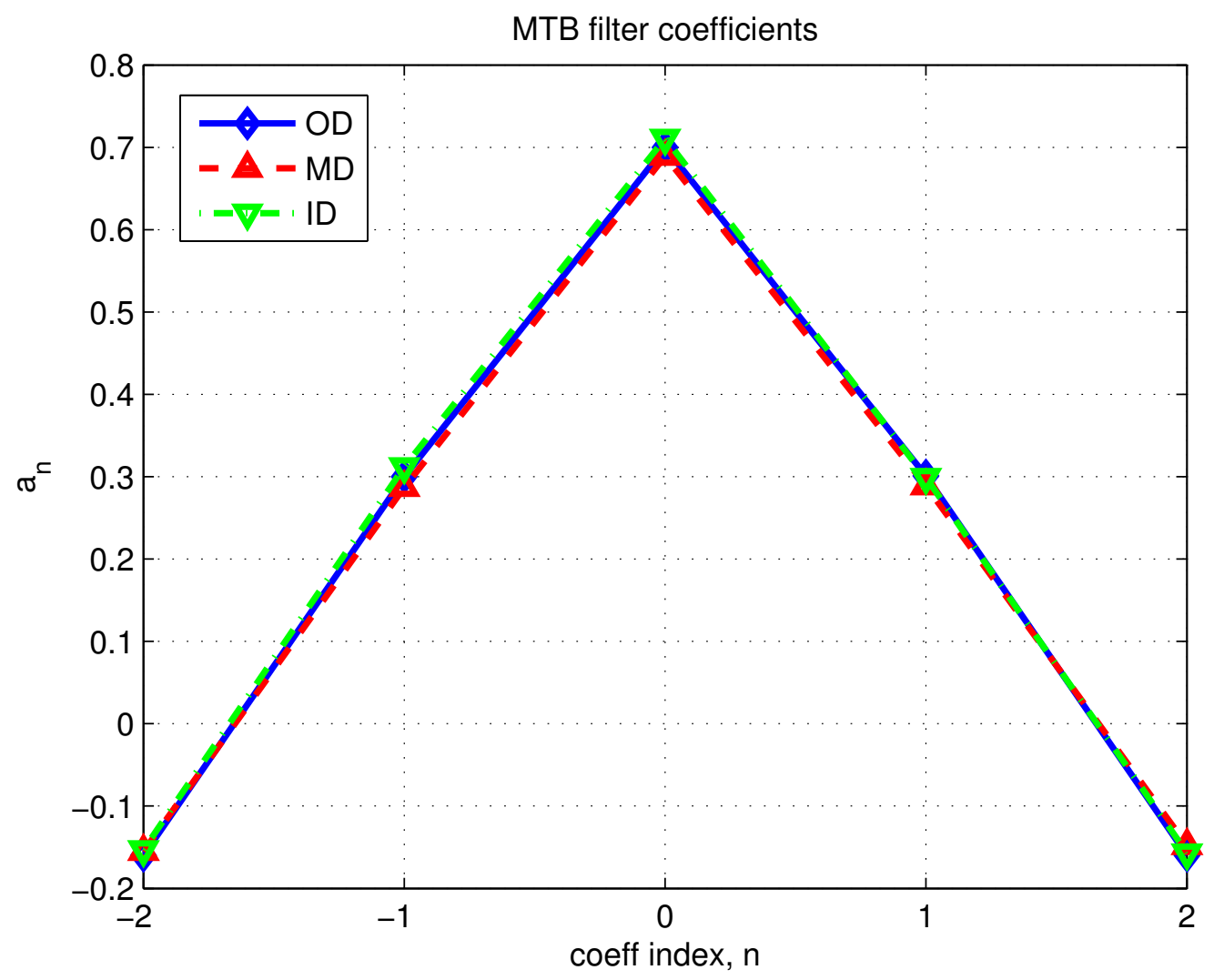

Figure 6.7: Example of MTB coefficients.

Figure 6.8 shows the TMR performance comparison between basic CWRRO scheme and MTB. MTB can improve the worst case $R R O_{3 \sigma}$ by trading-off the performance at the learning location of $+/-25 \%$ off-track. With the help of MTB, the worst case $\mathrm{RRO}_{3 \sigma}$ can match TMR performance of TWRRO at $7 \%$ off-track. In addition, MTB can also improve the average $R R O_{3 \sigma}$ across the whole surface as shown in Figure 6.9. This is achieved because the MTB is designed in a way such that the MSE between estimated RRO and true RRO is minimized. Figure 6.10 shows the improvement of MSE across the whole surface. The only drawback of MTB compare with the basic scheme is the increase of total learning time. 


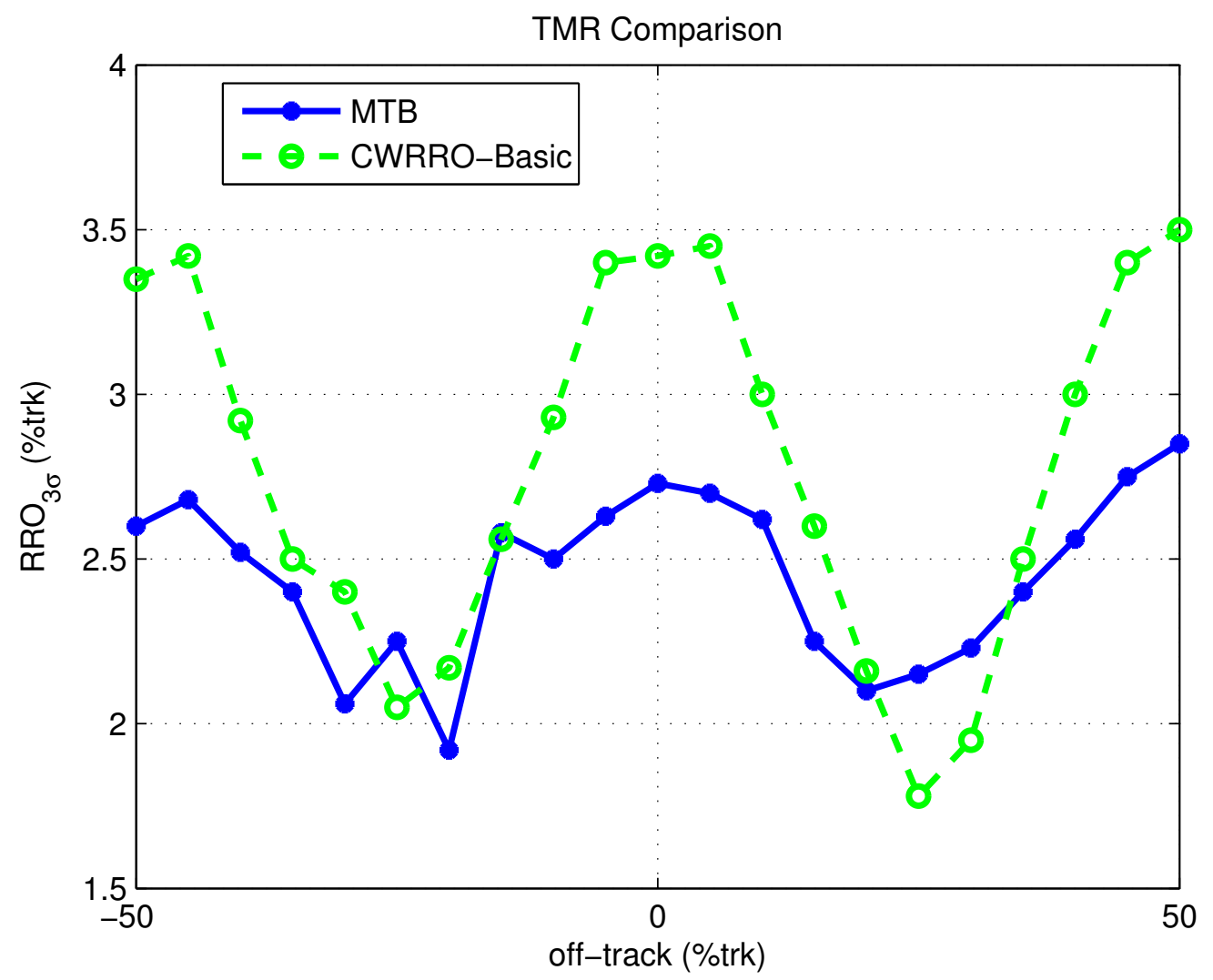

Figure 6.8: TMR comparison between CWRRO basic scheme and MTB.

\subsection{Conclusion}

The CWRRO solution is proposed to handle TWRRO off-track performance issue and improve the WRRO OTRC when areal density is beyond 1 terabyte/in ${ }^{2}$. The TMR performance of CWRRO can beat TWRRO if off-track is more than $7 \%$ trk. The major cost is the format efficiency loss due to the doubled payload compared with TWRRO, which has both of read-WRRO and write-WRRO. In the future, the better scheme should be developed to further improve the TMR performance without causing any further FE loss. In addition, efforts should be made to reduce the test time, which is one of the most important concerns in the mass production. 


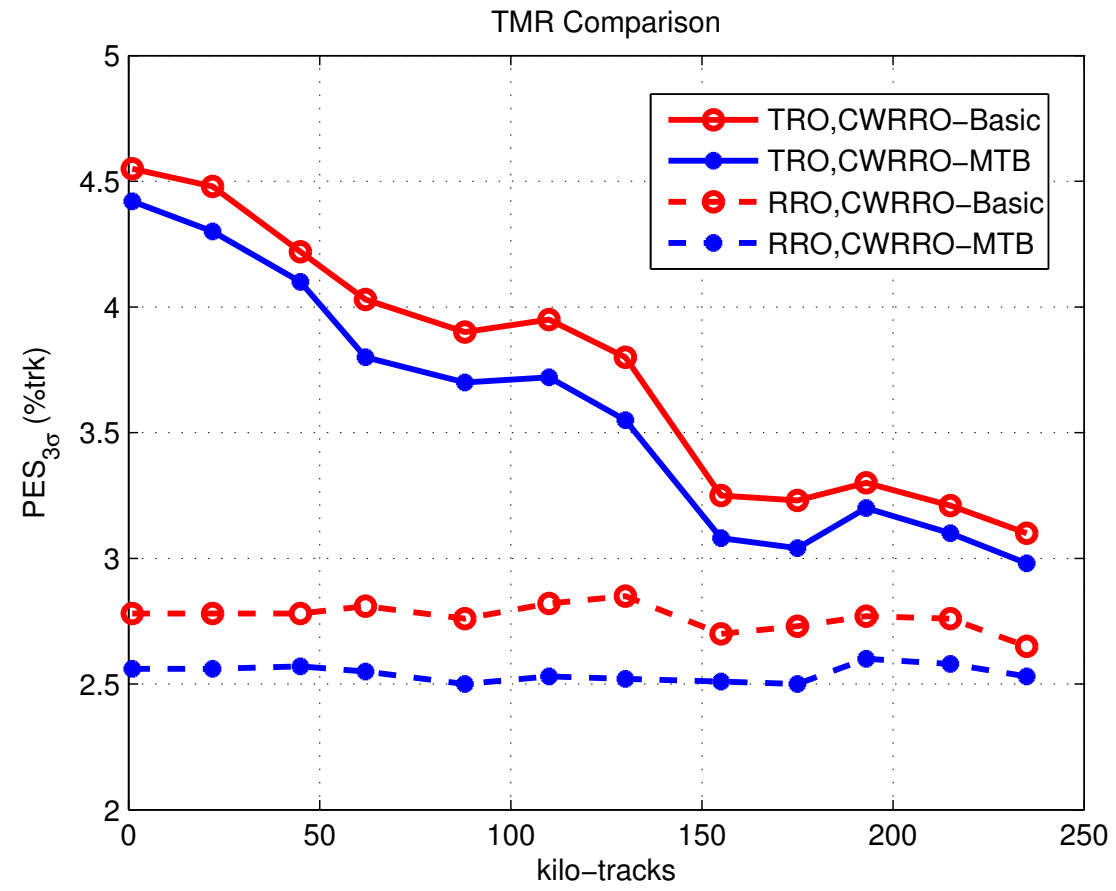

Figure 6.9: Cross surface average TMR comparison between CWRRO basic scheme and MTB.

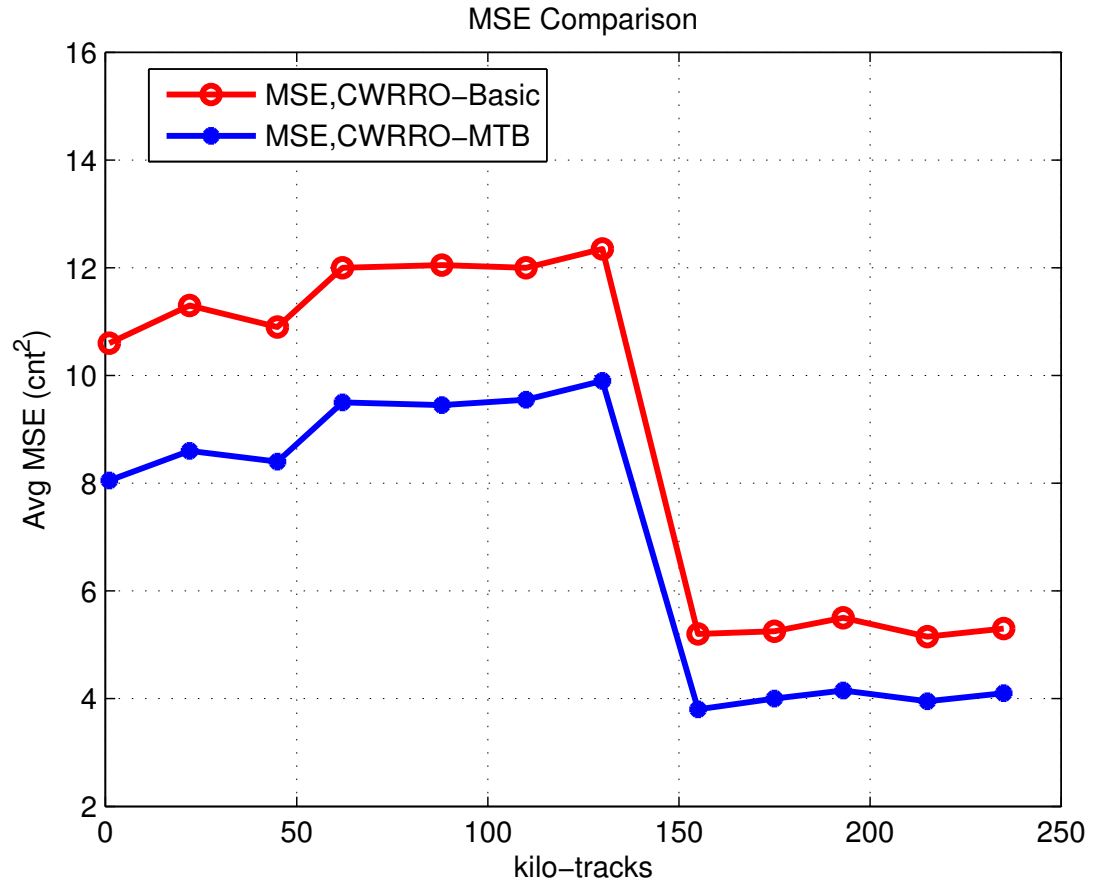

Figure 6.10: Cross surface residue MSE comparison between CWRRO basic scheme and MTB. 



\section{Chapter 7}

\section{Conclusions and Future Works}

\subsection{Conclusions}

Future HDDs could achieve the areal density of a few terabyte/in ${ }^{2}$, which will translate to more than one million track per inch. Much higher track density requires sophisticated track-following performance, which will exceed the capability of current HDD design. The HDD track-following performance can be improved from various aspects, such as the servo pattern quality including both of the burst pattern and RRO fields, the servo controller design, the mechanical design, the replacement of air with helium and so on. This thesis only focuses on the improvement of the servo pattern quality.

In Chapter 3 and 4, the main topics are the system modeling of the HAMR writing process and the applications of the model. This is essential for writing good quality servo patterns since the principle of writing technology for HAMR is fundamentally different from the PMR. The main challenges for HAMR are to design the laser light delivery system and the recording media. The areal density 
and written-in signal quality are determined by the thermal profile of the hot spot. Given the head and media design, the thermal profile is determined by the input laser power and the NFT to media spacing or NMS. Due to the laser-induced heat sources, the NMS keeps changing due to various protrusions, which makes the NMS control a very complex task. In addition, the heating process takes time for the temperature of the recording layer to reach its steady state. Using the dynamic model identified, the protrusion and thermal power delivered to the media under different conditions can be predicted. Then the optimized input laser power profile can be designed to write signal with good SNR from the very first bit of data. By doing so, the wasted FE by writing a long preamble to wait for signal quality to settle can be minimized. By using the protrusion output of the model, the various protrusions throughout the writing process (i.e, not limited to the steady state) can be predicted and NMS clearance can be maintained to avoid the head damage.

In Chapter 5, the ideal burst profile are identified, which should be symmetric and has a flatness of 0.82 . This kind of burst profile can minimize burst non-linearity, and the resulting peak-to-peak PES error is only $0.42 \%$ trk from the simulation. The shape of the burst profile can be controlled by adjusting the ratio between the reader width to servo track pitch. The PES is the only feedback signal in HDD to determine the head location for servo control. By reducing the PES demodulation error, the track-following performance will be improved.

In Chapter 6, a revolutionary HDD RRO compensation scheme is introduced, which is called continuous WRRO or CWRRO. RRO is the residue error or noise for every interested location after the PES demodulation correction process. They are random in nature and need to be learned for every servo sector and tracks. The TWRRO compensation scheme only learns and compensates for the RRO at data track center for read and write operation respectively. However, when the track pitch is significantly reduced, TWRRO performance will be badly degraded due 
to its poor OTRC. As its name suggests, CWRRO is capable of providing RRO compensation at any off-track locations. In order to achieve good performance, it is necessary to learn RRO compensation values at neighboring locations and do linear interpolation in between to approximate the RRO values for the locations in between. The lower non-linearity burst in Chapter 5 can help in this respect. In order to improve the TMR performance of the CWRRO scheme, a smaller distance between adjacent learning locations are desired. However, this also means that more data need to be stored in the media and a longer process time will be taken. As a result, a trade-off must be made between the TMR performance, format efficiency and the process time. Proposed MTB is to learn the RRO compensation values at more locations, and filter the compensation values to half the number of locations for storage. In this way, the worst TMR can be reduced without doubling the storage overhead.

With all of the above, the servo pattern quality can be improved, especially for HAMR drive, which, in turn, will improve the track-following performance of the future higher areal density HAMR HDDs.

\subsection{Suggestions for Future Research}

All the major HDD manufacturers have made tremendous progress in the HAMR technology. And prototypes of the HAMR drives are built and tested in the lab such as the one used for this research work. The HAMR drives are expected to reach the market in the coming years. With the continuous improvements on the head and media design, it is very important to evaluate their dynamic responses, especially in the drive level. The proposed HAMR writing process modeling discussed in Chapters 3 and 4 provide a good way to perform such evaluations. However, there are other areas for further improvements in order to improve the usefulness 
and accuracy of the model.

- Firstly, the highest writable frequency is determined by the disk rotation speed and thermal gradient in the down-track direction. As a result, in order to ignore the effect of the thermal profile in the down-track direction in our modeling, fairly low frequency single tone signals are written onto the media for the model identification process. However, the highest possible signal frequency for the recorded data is desired for higher areal density, and the effect of the down-track thermal profile should be considered in the modeling so that the dynamic response versus written-in signal frequency can be characterized. Higher written-in signal frequency can also improve the resolution of our model, which is determined by the period of the signal. The maximum thermal gradient is determined by the media and the writer head. However, when the read-back signal magnitude is measured, the effect of reader needs to be considered as well. One way to study the effect of down-track thermal profile is to write signal of different frequencies and then compare the read-back signal with those simulated from micro-magnetic simulations.

- In this thesis, a quadratic function is used to approximate the relationship between the media thermal power and written-in signal magnitude when the thermal power is small. This relationship enables us to estimate the media thermal power by measuring the signal magnitude since thermal power is not directly measurable. Although quadratic function is a good approximation, any deviation from the real situation will be transferred to the errors of identified model parameters. In another word, a better approximation is always desired in order to minimize the model identification errors. This becomes more important when the heat spot size and reader width become smaller and smaller. The relationship between the media thermal power and signal magnitude can be modeled more accurately with the help of micro-magnetic 
modeling. The new relationship can be incorporated into the model and then all the parameters can be identified as before. Finally, the comparison can be made between the new model output and the real drive measurement to see whether the residue error can be reduced.

- In Chapter 4, the input laser profile is optimized based on the signal magnitude response only. However, one of the biggest bottlenecks for HAMR is the lifetime of the NFT due to over-heating. In future, the temperature response of the NFT needs to be modeled, and the maximum NFT temperature should be limited while optimizing the input laser current in order to increase the lifetime of the NFT. As such, the problem can be formulated as a constrained optimization problem as opposed to the constrained command input shaping problem [30].

- In this thesis, the Gaussian-Newton method is used to identify the non-linear system by splitting it into a few sub-systems. The system identification [148$151]$ is a very big and complex topic. To find the best method to identify the model in this thesis by comparing different methods is very useful and may leads to better estimation results.

Both the burst pattern and CWRRO fields writing require stitched write in order to write a phase aligned pattern of bigger width than the magnetic write width. Since the written-in magnetic pattern is mainly determined by the thermal spot size for HAMR drive, the curvature effect of the written-in pattern in HAMR is more serious than PMR [183]. When the areal density is increased further, the heat spot size becomes smaller and smaller. And the curvature effect will become worse and worse. As a result, its impact on the quality of burst pattern and CWRRO field needs to be evaluated. And countermeasures need to be in place to tackle any adverse effects. 
For the CWRRO scheme, it is desired to find a better data compression scheme in order to store more useful RRO data in the media with lower format efficiency loss. In the meantime, the RRO correlation and linear interpolation performance for new generation head and media and servo writing technology are to be studied.

In addition, efforts must be made to reduce the processing time, which is one of the most important concerns in mass production. MTBs effectiveness on reducing the RRO estimation error is mainly due to the correlations of RROs among different locations. Although the high frequency RRO components have weak correlation, the level of correlation is high enough for adjacent RRO estimation if the distance is kept small such as half-track or quarter-track as shown in Figure 6.4. In HDDs, the RRO is most commonly learned through iterative learning control (ILC) or similar idea [42]. The basic approach is that the learning buffer is initialized to zeros and then filled with some learned values after first revolution. Then the buffer values are added with new learned values after multiplying some gain. This process continues until some convergence criteria is met or the maximum number of revolution is reached. The above procedure can be improved by initialized by some value learned from previous track instead of zeros. Since the adjacent learning location RROs contain some information about current learning location, the learning convergence rate could be improved. 


\section{Author's Publications - Journal}

1. Kun Ma, Wai Ee Wong, Jianyi Wang, Guoxiao Guo, and Youyi Wang, "Writing Process Modeling and Identification for Heat-Assisted Magnetic Recording," IEEE Trans. Magn., vol. 53, no. 2, pp. 1-7, Feb 2017.

2. Jianyi Wang, Kun Ma, Zhen Ye, Wai Ee Wong, Guoxiao Guo, and Youyi Wang, "HAMR Writing Process Model Based Compensation of Laser Induced Transients," IEEE Trans. Magn., vol. 53, no. 3, pp. 1-7, Mar 2017.

\section{Author's Publications - Conference}

1. Kun Ma, Wai Ee Wong, Jianyi Wang, Guoxiao Guo, and Youyi Wang, "Writing Process Modeling and Identification for Heat-Assisted Magnetic Recording," Dig. The 27th Magnetic Recording Conf., Stanford, CA, USA, Aug. 2016, A5 (invited).

2. Jianyi Wang, Kun Ma, Zhen Ye, Wai Ee Wong, Guoxiao Guo, and Youyi Wang, "Modelling and Compensation for Laser Induced Transients to Improve HAMR Writing Quality," Dig. Asia-Pacific Magnetic Recording Conf., Seoul, Korea, Jul. 2016, S1-01 (invited).

\section{Author's Patents}

1. Kun Ma, Jianyi Wang, Hui Li, Wai Ee Wong, and Guoxiao Guo, "Data Storage Device Filtering Burst Correction Values Before Downsampling the Burst Correction Values," U.S. Patent 9,343,094, May 17, 2016.

2. Hui Li, Jianyi Wang, Kun Ma, Shanjiao Jiang, Yanfeng Zhang, Jie Wan and Guoxiao Guo, "Data Storage Device Writing Scaled Burst Correction Values to a Disk," US Patent Application No. 14/887,140, Oct, 2015. 


\section{Bibliography}

[1] Wikipedia, "History of hard disk drives." https://en.wikipedia.org/wiki/ History_of_hard_disk_drives, 2016. Online: accessed 7-Sep-2016.

[2] Z. Z. Bandic and R. H. Victora, "Advances in magnetic data storage technologies," Proc. IEEE, vol. 96, no. 11, pp. 1749-1753, Nov 2008.

[3] H. Masakatsu, S. Hiroshi, and S. Seiichi, "Trends in technologies for HDDs, ODDs, and SSDs, and toshiba's approach," 2015.

[4] Y. Shiroishi, K. Fukuda, I. Tagawa, H. Iwasaki, S. Takenoiri, H. Tanaka, H. Mutoh, and N. Yoshikawa, "Future options for HDD storage," IEEE Trans. Magn., vol. 45, no. 10, pp. 3816-3822, Oct 2009.

[5] M. H. Kryder, E. C. Gage, T. W. McDaniel, W. A. Challener, R. E. Rottmayer, G. Ju, Y.-T. Hsia, and M. F. Erden, "Heat assisted magnetic recording," Proc. IEEE, vol. 96, no. 11, pp. 1810-1835, Nov. 2008.

[6] tom'sIT Pro, "Shingled Magnetic Recording 101." http://www.tomsitpro. com/articles/shingled-magnetic-recoding-smr-101-basics , 2-933. html, 2015. Online: accessed 7-Sep-2016.

[7] R. Wood, "Shingled magnetic recording and two-dimensional magnetic recording," IEEE SCV Magn. Soc., pp. 1-59, Oct 2010.

[8] M. Salo, T. Olson, R. Galbraith, R. Brockie, B. Lengsfield, H. Katada, and Y. Nishida, "The structure of shingled magnetic recording tracks," IEEE Trans. Magn., vol. 50, no. 3, pp. 18-23, Mar 2014.

[9] S. Chandrasekaran, P. Supnithi, C. Warisarn, and D. Bai, "Spinning disk test study on erase band and write width for shingled magnetic recording," $J$. Appl. Phys., vol. 115, no. 17B736, 2014. 
[10] R. Wood, M. Williams, A. Kavcic, and J. Miles, "The feasibility of magnetic recording at 10 terabits per square inch on conventional media," IEEE Trans. Magn., vol. 45, no. 2, pp. 917-923, Feb 2009.

[11] K. S. Chan, J. J. Miles, E. Hwang, B. V. K. VijayaKumar, J. Zhu, W. Lin, and R. Negi, "TDMR platform simulation and experiments," IEEE Trans. Magn., vol. 45, no. 10, pp. 3837-3843, Oct 2009.

[12] M. R. Elidrissi, K. S. Chan, K. K. Teo, K. Eason, E. Hwang, B. V. K. V. Kumar, and Z. Qin, "Modeling of 2-D magnetic recording and a comparison of data detection schemes," IEEE Trans. Magn., vol. 47, no. 10, pp. 36853690 , Oct 2011.

[13] E. Hwang, R. Negi, B. V. K. V. Kumar, and R. Wood, "Investigation of twodimensional magnetic recording (TDMR) with position and timing uncertainty at 4 Tb/in2," IEEE Trans. Magn., vol. 47, no. 12, pp. 4775-4780, Dec 2011.

[14] R. Wood, R. Galbraith, and J. Coker, "2-D magnetic recording: Progress and evolution," IEEE Trans. Magn., vol. 51, no. 4, Apr 2015.

[15] Toshiba, "Bit-patterned media for high-density hdds." http://www.toshiba. co.jp/rdc/rd/fields/11_e_09e.htm, 2011. Online: accessed 7-Sep-2016.

[16] T. Register, "Seagate ready for the hamr blow: First drives out in 2017." http://www.theregister.co.uk/2016/05/12/how_will_hamr_ technology_affect_seagate_in_derry/, 2016. Online: accessed 19-Oct2016 .

[17] K.-S. Park, Y.-P. Park, and N.-C. Park, "Prospect of recording technologies for higher storage performance," IEEE Trans. Magn., vol. 47, no. 3, pp. 539-545, Apr 2011.

[18] IDEMA, "2016 ASTC Technology Roadmap." http://idema.org/?page_id= 5868, 2016. Online: accessed 25-Oct-2016.

[19] E. Brewer, L. Ying, L. Greenfield, R. Cypher, and T. Ts'o, "Disks for data centers." White Paper, Oct Feb 2016.

[20] B. M. Lairson, S. E. Lambert, H. Nguy, L. Nguyen, A. Wallash, T. Huang, and J. Adler, "Disk drive integration issues for perpendicular recording," IEEE Trans. Magn., vol. 37, no. 4, pp. 1223-1227, Jul 2001. 
[21] W. Xi and S. Chu, "Alternating wedge repeatable runout (wrro) pattern." U.S. Patent 8,693,134, 2014.

[22] N. Yoshikawa, K. Titagawa, S. Takakura, and S. Takekado, "An experiment for head positioning system using submicron track-width GMR head," IEEE Trans. Magn., vol. 32, no. 5, pp. 3905-3907, Sep 1996.

[23] K. Ma, W. E. Wong, J. Wang, G. Guo, and Y. Wang, "Writing process modeling and identification for heat-assisted magnetic recording," IEEE Trans. Magn., vol. 53, no. 2, pp. 1-7, Feb 2017.

[24] J. Wang, K. Ma, Z. Ye, W. E. Wong, G. Guo, and Y. Wang, "Hamr writing process model based compensation of laser induced transients," IEEE Trans. Magn., vol. 53, no. 3, pp. 1-7, Mar 2017.

[25] A. Dhanda, W. Guo, R. A. Quisenberry, G. Guo, and J. Yu, "Disk drive evaluating burst metric." U.S. Patent 8,970,980, 2015.

[26] S. H. Shepherd, Y. M. Lifschitz, M. Chen, G. Guo, and J. Laks, "Data storage device writing multiple burst correction values at the same radial location." U.S. Patent 9,286,925, 2016.

[27] K. Ma, J. Wang, H. Li, W. E. Wong, and G. Guo, "Data storage device filtering burst correction values before downsampling the burst correction values." U.S. Patent 9,343,094, 2016.

[28] HGST, "Dual stage actuator." White Paper, Oct 2013.

[29] U. Boettcher, R. A. de Callafon, and F. E. Talke, "Modeling and control of a dual stage actuator hard disk drive," JSME, vol. 4, no. 1, pp. 107-118, 2010.

[30] A. Mamun, G. Guo, and C. Bi, Hard Disk Drive: Mechatronics and Control. CRC Press, Boca Raton, 2006.

[31] H. Nakanishi and M. Mizukami, "High track density head positioning using sector servos," IEEE Trans. Magn., vol. 19, no. 5, pp. 1698-1700, Sep 1983.

[32] A. H. Sacks, Position Signal Generation in Magnetic Disk Drives. PhD thesis, CMU, 1995.

[33] W. E. Wong, G. Guo, A. A. Mamun, W. Ye, and J. Zhang, "A position encoding and decoding scheme for ultra high density magnetic recording," Dig. Asia-Pacific Magnetic Recording Conf., pp. WE-P-08, Singapore, 2002. 
[34] T. Yamaguchi, "Modelling and control of a disk file head-positioning system," Proc. Instn. Mech. Engrs., vol. 215, Part I, no. 6, pp. 549-568, 2001.

[35] K. K. Chew and M. Tomizuka, "Digital control of repetitive errors in disk drive systems," IEEE Control Syst. Mag., vol. 10, no. 1, pp. 16-20, Jan 1990.

[36] A. Sacks, M. Bodson, and W. Messner, "Advanced methods for repeatable runout compensation," IEEE Trans. Magn., vol. 31, no. 2, pp. 1031-1036, Mar 1995.

[37] M. Kawafuku, M. Iwasaki, H. Hirai, M. Kobayashi, and A. Okuyama, "Rejection of repeatable runout for HDDs using adaptive filter," Proc. International Workshop Adv. Motion Control, pp. 305-310, Hiyoshi, Kohoku, Yokohama, Japan, Mar 2014.

[38] M. Bodson, A. Sacks, and P. Khosla, "Harmonic generation in adaptive feedforward cancellation schemes," IEEE Trans. Autom. Control, vol. 39, no. 9, pp. 1939-1944, Sep 1994.

[39] J. Zhang, R. Chen, G. Guo, and T. Low, "Modified adaptive feedforward runout compensation for dual-stage servo system," IEEE Trans. Magn., vol. 36, no. 5, pp. 3581-3584, Sep 1994.

[40] A. W. Nazarian, C. W. Simmons, T. P. Trieu, and R. K. Wong, "Repeatable runout cancellation in sectored servo disk drive positioning system." U.S. Patent 6,310,742, Oct 30, 2001.

[41] Y. Q. Chen, L. L. Tan, K. K. Ooi, Q. Bi, and K. H. Cheong, "Repeatable runout compensation using a learning algorithm with scheduled parameters." U.S. Patent 6,437,936, Aug 20, 2002.

[42] M. Chen and L. Yi, "Disk drive to estimate repeatable runout (RRO) based upon on an optimal mean square estimation (MSE) learning method." U.S. Patent 7,595,954, Sep 29, 2009.

[43] S.-C. Wu and M. Tomizuka, "Repeatable runout compensation for hard disk drives using adaptive feedforward cancellation," Proc. American Control Conf., pp. 382-387, Minneapolis, MN, USA, 2006.

[44] K. L. Moore, "Iterative learning control: An expository overview," Appl. Comput. Controls, Signal Process., Circuits, vol. 1, no. 1, pp. 425-488, 1998. 
[45] C. M. Cosner and S. F. Bockman, "Repetitive control of thermal shock disturbance." U.S. Patent 5,563,794, Oct 8, 1996.

[46] L. Guo, "A new disturbance rejection scheme for hard disk drive control," Proc. American Control Conf., pp. 1553-1557, Philadelphia, PA, USA, 1998.

[47] J. C. Morris and T. C. Zirps, "Compression and storage of written-in error compensation tables in an embedded servo disk drive." U.S. Patent 6,449,116, Sep 10, 2002.

[48] J. C. Morris, B. R. Pollock, and T. F. Ellis, "Compensation for repeatable run-out error." U.S. Patent 6,069,764, May 30, 2000.

[49] S. H. Shepherd, R. M. Ehrlich, J. V. Wiseman, R. T. Curran, and W. R. Akin, "In-drive correction of servo pattern errors." U.S. Patent 6,061,200, May 9, 2000.

[50] D. Wu, G. Guo, and T. C. Chong, "Midfrequency disturbance suppression via micro-actuator in dual-stage HDDs," IEEE Trans. Magn., vol. 38, no. 5, pp. 2189-2191, Sep 2002.

[51] S. M. Sri-Jayantha, H. Dang, A. Sharma, I. Yoneda, N. Kitazaki, and S. Yamamoto, "Truetrack servo technology for high TPI disk drives," IEEE Trans. Magn., vol. 37, no. 2, pp. 871-876, Mar 2001.

[52] M. Bodson, J. S. Jensen, and S. C. Douglas, "Active noise control for periodic disturbances," IEEE Trans. Control Syst. Technol., vol. 9, no. 1, pp. 200-205, Jan 2001.

[53] Q. Jia, Z. Wang, and F. Wang, "Repeatable runout disturbance compensation with a new data collection method for hard disk drive," IEEE Trans. Magn., vol. 41, no. 2, pp. 791-796, Feb 2005.

[54] R. D. Murphy, L. R. McKenzie, and R. D. Hampshire, "Dynamic compensation of servo burst measurement offsets in a disc drive." U.S. Patent 5,835,300, Nov 10, 1998.

[55] N. A. Spaldin, Magnetic Materials: Fundamentals and Device Applications. Cambridge University Press, Cambridge, UK, 2003.

[56] T. W. McDaniel, "Ultimate limits to thermally assisted magnetic recording," J. Phys.: Condens. Matter, vol. 17, no. 7, pp. R315-R332, Feb 2005. 
[57] A. Q. Wu, Y. Kubota, T. Klemmer, T. Rausch, Y. P. C. Peng, D. Karns, X. Zhu, Y. Ding, E. K. C. Chang, Y. Zhao, H. Zhou, K. Gao, J. Thiele, M. Seigler, G. Ju, and E. Gage, "HAMR areal density demonstration of $1+$ Tbpsi on spinstand," IEEE Trans. Magn., vol. 49, no. 2, pp. 779-782, Feb 2013.

[58] G. Ju, Y. Peng, E. K. C. Chang, Y. Ding, A. Q. Wu, X. Zhu, Y. Kubota, T. J. Klemmer, H. Amini, L. Gao, Z. Fan, T. Rausch, P. Subedi, M. Ma, S. Kalarickal, C. J. Rea, D. V. Dimitrov, P. Huang, K. Wang, X. Chen, C. Peng, W. Chen, J. W. Dykes, M. A. Seigler, E. C. Gage, R. Chantrell, and J. Thiele, "High density heat-assisted magnetic recording media and advanced characterization progress and challenges," IEEE Trans. Magn., vol. 51, no. 11, pp. 1-9, Nov 2015.

[59] R. E. Rottmayer, S. Batra, D. Buechel, W. A. Challener, J. Hohlfeld, Y. Kubota, L. Li, B. Lu, C. Mihalcea, K. Mountfield, K. Pelhos, C. Peng, T. Rausch, M. A. Seigler, D. Weller, and X. Yang, "Heat-assisted magnetic recording," IEEE Trans. Magn., vol. 42, no. 10, pp. 2417-2421, Oct 2006.

[60] S. Greaves, Y. Kanai, and H. Muraoka, "Magnetization switching in energy assisted recording," IEEE Trans. Magn., vol. 48, no. 5, pp. 1794-1800, May 2012.

[61] D. Weller and A. Moser, "Thermal effect limits in ultrahigh-density magnetic recording," IEEE Trans. Magn., vol. 35, no. 6, pp. 4423-4439, Nov 1999.

[62] D. Weller, A. Moser, L. Folks, M. E. Best, W. Lee, M. F. Toney, M. Schwickert, J.-U. Thiele, and M. F. Doerner, "High $K_{u}$ materials approach to 100 Gbits/in'," IEEE Trans. Magn., vol. 36, no. 6, pp. 10-15, Jan 2000.

[63] T. Klemmer, D. Hoydick, H. Okumura, B. Zhang, and W. A. Soffa, "Magnetic hardening and coercivity mechanisms in $\mathrm{L}_{0}$ ordered FePd ferromagnets," Scripta Metallurgica et Materialia, vol. 33, no. 10-11, pp. 1793-1805, 1995.

[64] J. Sayama, K. Mizutani, T. Asahi, and T. Osaka, "Thin films of $S_{m} C_{o 5}$ with very high perpendicular magnetic anisotropy," Appl. Phys. Lett., vol. 85, pp. 5640-5642, 2004.

[65] Y. K. Takahashi, T. Ohkubo, and K. Hono, "Microstructure and magnetic properties of $S_{m} C_{o 5}$ thin films deposited on $\mathrm{Cu}$ and Pt underlayers," J. Appl. Phys., vol. 100, no. 053913, 2006. 
[66] J. Sayama, K. Mizutani, T. Asahi, J. Ariake, K. Ouchi, S. Matsunuma, and T. Osaka, "Magnetic properties and microstructure of $S_{m} C_{o 5}$ thin film with perpendicular magnetic anisotropy," J. Magn. Mater., vol. 287, no. SPEC.ISS, pp. 239-244, Feb 2005.

[67] Y. Peng, T. J. Klemmer, G. Ju, E. Gage, M. A. Seigler, W. A. Challener, D. C. Karns, X. Zhu, N. Gokemeijer, C. Peng, K. Pelhos, B. Lu, T. Rausch, X. W. Wu, L. Li, Y. T. Hsia, D. Buechel, R. D. Hempstead, and R. Rottmayer, "Heat assisted magnetic recording on high anisotropy nanocomposite media," Proc. IEEE Conf. Nanotechnol. (NANO), pp. 603-604, Arlington, Texas, USA, Aug 2008.

[68] T. Klemmer, Y. Peng, X. Wu, and G. Ju, "Materials processing for high anosotropy L1 $1_{0}$ granular media," IEEE Trans. Magn., vol. 45, no. 2, pp. 845849, Feb 2009.

[69] Y. Kubota, X. Zhu, E. K. Chang, Y. Peng, Y. Ding, T. Klemmer, L. Gao, A. Q. Wu, H. Amini, G. Ju, and J.-U. Thiele, "Thermal design for heat assisted magnetic recording media," Dig. The 25th Magnetic Recording Conf., p. D2, Berkeley, CA, USA, 2014.

[70] R. Acharya, "Challenges and recent development of heat assisted magnetic recording media," Dig. The 25th Magnetic Recording Conf., p. D3, Berkeley, CA, USA, 2014.

[71] D. Weller, O. Mosendz, G. Parker, S. Pisana, and T. Santos, "L1 0 FePtX-Y media for heat-assisted magnetic recording," Phys. Status Solidi A, vol. 210, no. 7 , pp. $1245-1260,2013$.

[72] J. S. Chen, B. C. Lim, J. F. Hu, B. Liu, G. M. Chow, and G. Ju, "Low temperature deposited L10 FePt-C (001) films with high coercivity and small grain size," Appl. Phys. Lett., vol. 91, no. 132506, 2007.

[73] A. Perumal, Y. K. Takahashi, and K. Hono, "L1 $1_{0}$ FePt-C nanogranular perpendicular anisotropy films with narrow size distribution," Appl. Phys. Expr., vol. 1, no. 10, p. 101301, Sep 2008.

[74] E. Yang and D. E. Laughlin, "L1 $1_{0}$ FePt-oxide columnar perpendicular media with high coercivity and small grain size," J. Appl. Phys., vol. 104, no. 023904, July 2008. 
[75] B. Valcu, T. Seki, A. Chernyshov, F. Zong, and A. Ajan, "Thermal layers effect on recording performance in HAMR," IEEE Trans. Magn., vol. 50, no. 11, pp. 1-4, Nov 2014.

[76] N. Zhou, X. Xu, A. T. Hammack, B. C. Stipe, K. Gao, W. Scholz, and E. C. Gage, "Plasmonic near-field transducer for heat-assisted magnetic recording," Nanophotonics, vol. 3, no. 3, pp. 141-155, Jun 2014.

[77] W. A. Challener, C. Peng, A. V. Itagi, D. Karns, W. Peng, Y. Peng, X. Yang, X. Zhu, N. J. Gokemeijer, Y. Hsia, G. Ju, R. E. Rottmayer, M. A. Seigler, and E. C. Gage, "Heat-assisted magnetic recording by a near-field transducer with efficient optical energy transfer," Nat. Photon., vol. 3, pp. 220-224, Mar 2009 .

[78] B. C. Stipe, T. C. Strand, C. C. Poon, H. Balamane, T. D. Boone, J. A. Katine, J.-L. Li, V. Rawat, H. Nemoto, A. Hirotsune, O. Hellwig, R. Ruiz, E. Dobisz, D. S. Kercher, N. Robertson, T. R. Albrecht, and B. D. Terris, "Magnetic recording at $1.5 \mathrm{~Pb} \mathrm{~m}-2$ using an integrated plasmonic antenna," Nat. Photon., vol. 4, pp. 484-488, May 2010.

[79] T. Matsumono, F. Akagi, M. Mochizuki, H. Miyamoto, and B. Stipe, "Integrated head design using a nanobeak antenna for thermally assisted magnetic recording," Opt. Exp., vol. 20, no. 17, pp. 18946-18954, Aug 2012.

[80] L. Huang, B. Stipe, M. Staffaroni, J. Juang, T. Hirano, E. Schreck, and F. Huang, "HAMR thermal modeling including media hot spot," IEEE Trans. Magn., vol. 49, no. 6, pp. 2565-2568, Jun 2013.

[81] T. Thio, K. M. Pellerin, and R. A. Linke, "Enhanced light transmission through a single subwavelength aperture," Opt. Lett., vol. 26, no. 24, pp. 19721974, Dec 2001.

[82] E. Betzig, J. K. Trautman, T. D. Harris, J. S. Weiner, and R. L. Kostelak, "Breaking the diffraction barrier: Optical microscopy on a nanometric scale," Science, vol. 251, no. 5000, pp. 1468-1470, Mar 1991.

[83] E. Betzig, J. K. Trautman, R. Wolfe, E. M. Gyorgy, P. L. Finn, M. H. Kryder, and C. H. Chang, "Near-field magneto-optics and high density data storage," Appl. Phys. Lett., vol. 61, pp. 142-144, 1992. 
[84] R. Wannemacher, "Plasmon-supported transmission of light trhough nanometric holds in metallic thin films," Opt. Commun., vol. 195, no. 1-4, pp. 107$118,2001$.

[85] W. A. Challener, T. W. McDaniel, C. D. Mihalcea, K. R. Mountfield, K. Pelhos, and I. K. Sendur, "Light delivery techniques for heat-assisted magnetic recording," Jpn. J. Appl. Phys., vol. 42, no. 2B, p. 981, Feb 2003.

[86] W. A. Challener, E. Gage, A. Itagi, and C. Peng, "Optical transducers for near field recording," Jpn. J. Appl. Phys., vol. 45, no. 8B, p. 6632, Aug 2006.

[87] A. V. Itagi, D. D. Stancil, J. A. Bain, and T. E. Schlesinger, "Ridge waveguide as a near-field optical source," Appl. Phys. Lett., vol. 83, pp. 4474-4476, 2003.

[88] K. Endur, W. Challener, and C. Peng, "Ridge waveguide as a near field aperture for high density data storage," J. Appl. Phys., vol. 96, no. 5, pp. 27432752, Sep 2004.

[89] Y. C. Chen, J. Y. Fang, C. H. Tien, and H. P. D. Shieh, "High-transmission hybrid-effect-assisted nanoaperture," Opt. Lett., vol. 31, no. 5, pp. 655-657, Mar 2006.

[90] E. X. Jin and X. Xu, "Obtaining super resolution light spot using surface plasmon assisted sharp ridge nanoaperture," Appl. Phys. Lett., vol. 86, no. 111106, 2005.

[91] X. Shi, R. L. Thornton, and L. Hesselink, "A nano-aperture with 1000x power throughput enhancement for very small aperture laser system (VSAL)," Proc. SPIE, vol. 4342, pp. 320-327, 2002.

[92] T. E. Schlesinger, T. Rausch, A. Itagi, J. Zhu, J. A. Bain, and D. D. Stancil, "An integrated read/write head for hybrid recording," Jpn. J. Appl. Phys., vol. 41, no. 3B, p. 1821, 2002.

[93] K. Sendur, C. Peng, and W. Challener, "Near-field radiation from a ridge waveguide transducer in the vicinity of a solid immersion lens," Phys. Rev. Lett., vol. 94, no. 4, p. 043901, Jan 2005.

[94] L. Wang, S. M. Uppuluri, E. X. Jin, and X. Xu, "Nanolighography using high transmission nanoscale bowtie apertures," Nano. Lett., vol. 6, no. 3, pp. 361364, 2006. 
[95] J. Xu, T. Xu, J. Wang, and Q. Tian, "Design tips of nanoapertures with strong field enhancement and proposal of novel L-shaped aperture," Opt. Eng., vol. 44, no. 1, p. 018001, Dec 2004.

[96] M. A. Seigler, W. A. Challener, E. Gage, N. Gokemeijer, G. Ju, B. Lu, K. Pelhos, C. Peng, R. E. Rottmayer, X. Yang, H. Zhou, and T. Rausch, "Integrated heat assisted magnetic recording head: Design and recording demonstration," IEEE Trans. Magn., vol. 44, no. 1, pp. 119-124, Jan 2008.

[97] T. Matsumoto, Y. Anzai, T. Shintani, K. Nakamura, and T. Nishida, "Writing 40nm marks by using a beaked metallic plate near-field optical probe," Opt. Lett., vol. 31, no. 2, pp. 259-261, Jan 2006.

[98] G. V. Naik, J. Kim, and A. Boltasseva, "Oxides and nitrides as alternative plasmonic materials in the optical range," Opt. Master. Exp., vol. 1, no. 6, pp. 1090-1099, 2011.

[99] T. Zhao, M. C. Kautzky, W. A. Challener, and M. A. Seigler, "HAMR NFT materials with improved thermal stability." U.S. Patent 8,427,925, Apr 23, 2013 .

[100] D. A. Bobb, G. Zhu, M. Mayy, A. V. Gavrilenko, P. Mead, V. I. Gavrilenko, and M. A. Noginow, "Engineering of low-loss metal for nano-plasmonic and metamaterials applications," Appl. Phys. Lett., vol. 95, no. 151102, 2009.

[101] P. R. West, S. Ishii, G. V. Naik, V. M. Shalaev, and A. Boltasseva, "Searching for better plasmonic materials," Laser and Photonics Rev., vol. 4, no. 6, pp. 795-808, Nov 2010.

[102] G. V. Naik, J. Liu, A. V. Kildishev, V. M. Shalaev, and A. Boltasseva, "Demonstration of al:zno as a plasmonic component for near-infrared metamaterials," Proc. Natl. Acad. Sci. USA, vol. 109, no. 23, pp. 8834-8838, Mar 2012 .

[103] G. V. Naik, V. M. Shalaev, and A. Boltasseva, "Alternative plasmonic materials: beyond gold and silver," Adv. Mater., vol. 25, no. 24, pp. 3264-3294, May 2013.

[104] G. Zhu, L. Gu, J. K. Kitur, A. Urbas, J. Wella, and M. A. Noginov, "Organic materials with negative and controllable electric permittivity," Proc. Lasers and Electro-Optics (CLEO) Conf., Baltimore, Maryland, USA, 1-6 May 2011. 
[105] J. D. Kiely, P. M. Jones, H. Wang, R. Yang, W. Scholz, M. Benakli, J. L. Brand, and S. Gangopadhyay, "Media roughness and head-media spacing in heat-assisted magnetic recording," IEEE Trans. Magn., vol. 50, no. 3, pp. 132$136,2014$.

[106] D. Li, M. Staffaroni, E. Schreek, and B. Stipe, "A new AFM-based technique to detect the NFT protrusion on HAMR head," IEEE Trans. Magn., vol. 49, no. 7, pp. 3576-3579, Jul 2013.

[107] Z. Zhang, K. Liu, E. X. Jin, M. M. Dovek, J. D. Kiely, O. Nakada, P. K. Wong, V. M. F. Chiah, and X. M. Liu, "Characterization of laser-induced protrusion in HAMR by the burnish method," IEEE Trans. Magn., vol. 52, no. 2, pp. 1-6, Feb 2016.

[108] E. Schreck, D. Li, S. V. Canchi, L. Huang, G. P. Singh, B. Marchon, H. J. Richter, B. Stipe, and M. Staffaroni, "Thermal aspects and static/dynamic protrusion behaviors in heat-assisted magnetic recording," IEEE Trans. Magn., vol. 50, no. 3, pp. 126-131, Mar 2014.

[109] T. Rausch, J. D. Trantham, A. S. Chu, H. Dakroub, J. W. Riddering, C. P. Henry, J. D. Kiely, E. C. Gage, and J. W. Dykes, "HAMR drive performance and integration challenges," IEEE Trans. Magn., vol. 49, no. 2, pp. 730-733, Feb 2013 .

[110] H. Zheng, H. Li, and F. E. Talke, "Numerical simulation of thermal flying height control sliders in heat-assisted magnetic recording," Microsyst. Technol., vol. 18, no. 9, pp. 1731-1739, Sep 2012.

[111] M. F. Erden, T. Rausch, and W. A. Challener, "Cross-track transition location and transition parameter effects in heat-assisted magnetic recording," IEEE Trans. Magn., vol. 41, no. 6, pp. 2189-2194, Jun 2005.

[112] S. Xiong and D. B. Bogy, "Experimental study of head-disk interface in heatassisted magnetic recording," IEEE Trans. Magn., vol. 50, no. 3, pp. 148-154, Mar 2014.

[113] R. H. Victora and P.-W. Huang, "Simulation of heat-assisted magnetic recording using renormalized media cells," IEEE Trans. Magn., vol. 49, no. 2, pp. 751-757, Feb 2013. 
[114] M. Benakli, A. F. Torabi, M. L. Mallary, H. Zhou, and H. N. Bertram, "Micromagnetic study of switching speed in perpendicular recording media," IEEE Trans. Magn., vol. 37, no. 4, pp. 1564-1566, Jul 2001.

[115] Y. Jiao, Y. Wang, and R. H. Victora, "A study of SNR and BER in heatassisted magnetic recording," IEEE Trans. Magn., vol. 51, no. 11, pp. 1-4, Nov 2015.

[116] J. Zhu and H. Li, "Medium optimization for lowering head field and heating requirements in heat-assisted magnetic recording," IEEE Magn. Lett., vol. 6, pp. 1-4, 2015.

[117] H. Li, M. Alex, and J. Zhu, "HAMR noise mechanism study with spin-stand testing," IEEE Trans. Magn., vol. 51, no. 11, pp. 1-4, Nov 2015.

[118] S. Bhargava and E. Yablonovitch, "Lowering HAMR near-field transducer temperature via inverse electromagnetic design," IEEE Trans. Magn., vol. 51, no. 4, pp. 1-7, Apr 2015.

[119] X. Xu, N. Zhou, Y. Li, and L. Traverso, "Optical and thermal behaviors of plasmonic bowtie aperture and its NSOM characterization for heat-assisted magnetic recording," IEEE Trans. Magn., vol. 52, no. 2, pp. 1-5, Feb 2016.

[120] B. Xu, Y. T. Toh, C. W. Chia, J. Li, J. Zhang, K. Ye, and C. An, "Relationship between near field optical transducer laser absorption and its efficiency," IEEE Trans. Magn., vol. 48, no. 5, pp. 1789-1793, May 2012.

[121] B. X. Xu, Z. J. Liu, R. Ji, Y. T. Toh, J. F. Hu, J. M. Li, J. Zhang, K. D. Ye, and C. W. Chia, "Thermal issues and their effects on heat-assisted magnetic recording system," J. Appl. Phys., vol. 111, no. 07B701, Feb 2012.

[122] X. Wang, K. Gao, H. Zhou, A. Itagi, M. Seigler, and E. Gage, "HAMR recording limitation and extendibility," IEEE Trans. Magn., vol. 49, no. 2, pp. 686692, Feb 2013.

[123] X. Wang, K. Gao, and M. Seigler, "Magnetization switching variations and transition-width limit in heat-assisted magnetic recording," IEEE Trans. Magn., vol. 47, no. 10, pp. 2371-2374, Oct 2011.

[124] A. F. Torabi, J. V. Ek, E. Champion, and J. Wang, "Micromagnetic modeling study of thermal gradient effect in heat-assisted magnetic recording (HAMR)," IEEE Trans. Magn., vol. 45, no. 10, pp. 3848-3850, Oct 2009. 
[125] J. Barker, R. F. L. Evans, R. W. Chantrell, D. Hinzke, and U. Nowak, "Atomistic spin model simulation of magnetic reversal modes near the curie point," Appl. Phys. Lett., vol. 97, no. 192504, 2010.

[126] Y. Wang and J. Zhu, "Understanding field angle for heat assisted magnetic recording via dynamic modeling," Appl. Phys. Lett., vol. 109, no. 07B706, 2011.

[127] P. Huang, X. Chen, and R. H. Victora, "Time dependence of magnetic anisotropy at finite temperature for homogeneous and composite media," IEEE Trans. Magn., vol. 48, no. 11, pp. 3188-3191, Nov 2012.

[128] J.-G. Zhu and H. Li, "Understanding signal and noise in heat assisted magnetic recording," IEEE Trans. Magn., vol. 49, no. 2, pp. 765-772, Feb. 2013.

[129] S. H. Vemuri, H. M. Kim, S. Park, Y. Liu, P. S. Chung, and M. S. Jhon, "Thermal management in heat-assisted magnetic recording," IEEE Trans. Magn., vol. 50, no. 11, pp. 1-4, Nov 2014.

[130] S. Pisana, S. Jain, J. W. Reiner, O. Mosendz, G. J. Parker, M. Staffaroni, O. Hellwig, and B. C. Stipe, "Curie temperature distribution in FePt granular media," IEEE Trans. Magn., vol. 51, no. 4, pp. 1-5, Apr 2015.

[131] S. Pisana, S. Jain, J. W. Reiner, G. J. Parker, C. C. Poon, O. Hellwig, and B. C. Stipe, "Measurement of the curie temperature distribution in FePt granular magnetic media," Appl. Phys. Lett., vol. 104, no. 162407, 2014.

[132] S. H. Vemuri, W. Song, H. M. Kim, and M. S. Jhon, "A hybrid model for layer thermal management in heat-assisted magnetic recording," IEEE Trans. Magn., vol. 51, no. 11, pp. 1-4, Nov 2015.

[133] M. Chapline, C. Papusoi, A. Ajan, P. Dorsey, M. Desai, and R. Acharya, "Characterization of the magnetic properties of HAMR media using the temperature dependence of the AC susceptibility," IEEE Trans. Magn., vol. 52, no. 2, pp. 1-4, Feb 2016.

[134] J. E. Martin, M. V. Lubarda, V. Lomakin, and P. Jubert, "Effect of thermal fluctuations on the performance of particular media," IEEE Trans. Magn., vol. 49, no. 7, pp. 3137-3140, Jul 2013.

[135] C. Rea, M. Benakli, P. Subedi, R. Mendonsa, S. Kalarickal, J. Kiely, W. Chen, H. Zhou, S. Hernandez, Y. Peng, J. Thiele, A. Q. Wu, G. Ju, T. Rauch, K. Gao, 
M. Seigler, and E. Gage, "Writer and reader head-to-media spacing sensitivity assessment in HAMR," IEEE Trans. Magn., vol. 52, no. 2, pp. 1-6, Feb 2016.

[136] K. Sendur and P. Jones, "Effect of fly height and refractive index on the transmission efficiency of near-field optical transducers," Appl. Phys. Lett., vol. 88, no. 091110, 2006.

[137] U. Boettcher, H. Li, R. A. de Callafon, and F. E. Talke, "Dynamic flying height adjustment in hard disk drives through feedforward control," IEEE Trans. Magn., vol. 47, no. 7, pp. 1823-1829, 2011.

[138] P. A. Salas and F. E. Talke, "Numerical simulation of thermal flying-height control sliders to dynamically minimize flying height variations," IEEE Trans. Magn., vol. 49, no. 4, pp. 1337-1342, Apr 2013.

[139] P. A. Salas, U. Boettcher, and F. E. Talke, "Time dependent simulation of active flying height control of TFC sliders," Microsyst. Technol., vol. 18, no. 9, pp. 1661-1667, Jul 2012.

[140] S. Fukui, R. Wakabayashi, and H. Matsuoka, "Static flying characteristics of heat-assisted magnetic recording heads in he-enclosed HDDs," IEEE Trans. Magn., vol. 50, no. 11, pp. 1-4, Nov 2014.

[141] HGST, "Thermal fly-height control (TFC) technology in HGST hard disk drives." White Paper, 2007.

[142] A. Wallash, M. Salo, J. K. Lee, D. Heim, and G. Garfunkel, "Dependence of magnetoresistive head readback characteristics on sensor height," J. Appl. Phys., vol. 69, no. 8, pp. 5402-5404, Apr 1991.

[143] A. A. Kinkhabwala, M. Staffaroni, O. Suzer, S. Burgos, and B. Stipe, "Nanoscale thermal mapping of HAMR heads using polymer imprint thermal mapping," IEEE Trans. Magn., vol. 52, no. 2, pp. 1-4, Feb 2016.

[144] B. Xu, J. Li, Y.-T. Toh, K. Ye, and J. Zhang, "Dynamic thermal responses of heat-assisted magnetic recording head in data writing process," IEEE Trans. Magn., vol. 48, no. 11, pp. 3280-3283, Nov 2012.

[145] R. Conway, S. Felix, and R. Horowitz, "Model reduction and parametric uncertainty identification for robust $\mathrm{H} 2$ control synthesis for dual-stage hard disk drives," IEEE Trans. Magn., vol. 43, no. 9, pp. 3763-3768, 2007. 
[146] F. J. Macwilliams and N. J. A. Sloane, "Pseudo-random sequences and arrays," Proc. IEEE, vol. 64, no. 12, pp. 1715-1729, Dec 1976.

[147] D. Palmer and P. Ziperovich, "Identification of nonlinear write effects using pseudorandom sequences," IEEE Trans. Magn., vol. 23, no. 5, pp. 2377-2379, Oct 1987.

[148] W. Khalil and E. Dombre, Modeling, Identificaiton and Control of Robots. Kogan Page Science, London, 2004.

[149] L. Ljung and T. Glad, Modeling of Dynamic Systems. Prentice Hall, Inc., Englewood Cliffs, NJ, 1994.

[150] R. Johansson, System Modeling and Identification. Prentice Hall, Inc., Englewood Cliffs, NJ, 1993.

[151] L. Ljung, System Identification: Theory for the User. Prentice Hall, Inc., Englewood Cliffs, NJ, 1987.

[152] H. Li and J.-G. Zhu, "The role of media property distribution in HAMR SNR," IEEE Trans. Magn., vol. 49, no. 7, pp. 3568-3571, Jul 2013.

[153] C. Vogler, C. Abert, F. Bruckner, D. Suess, and D. Praetorius, "Basic noise mechanisms of heat-assisted-magnetic recording," cond-mat.mtrl-sci, 1605.00067v1, Apr 2006.

[154] S. Hernandez, P. Krivosik, P.-W. Huang, W. R. Eppler, T. Rausch, and E. Gage, "Parametric comparison of modeled and measured heat-assisted magnetic recording using a common signal-to-noise metric," IEEE Trans. Magn., vol. 52, no. 7, pp. 1-4, Jul 2016.

[155] O. Hovorka, S. Devos, Q. Coopman, W. J. Fan, C. J. Aas, R. F. L. Evans, X. Chen, G. Ju, and R. W. Chantrell, "The curie temperature distribution of FePt granular magnetic recording media," Appl. Phys. Lett., vol. 101, no. $052406,2012$.

[156] S. Xiong and D. B. Bogy, "Investigation of the local temperature increase for heat assisted magnetic recording (HAMR)," IEEE Trans. Magn., vol. 50, no. 4, pp. 1-6, Apr 2014.

[157] G. Guo and J. Yu, "Servo format trend and servo channel improvement for high areal density," Dig. Asia-Pacific Magnetic Recording Conf., pp. 1-2, Singapore, 2010. 
[158] C. L. Ong, S. Ang, and Z. Yuan, "Dynamic writer pole-tip protrusion compensation by thermal actuator," IEEE Trans. Magn., vol. 48, no. 11, pp. 45984601, Nov 2012.

[159] N. Wiener, Extrapolation, Interpolation, and Smoothing of Stationary Time Series, with Engineering Applications. Martino Fine Books, Eastford, CT, 2013.

[160] P. Haralson, H. Ruan, G. Chia, P. Thayamballi, K. Anderson, W. Cain, S. Dahandeh, J. Alexander, C. Macchioni, and G. Bertero, "Hamr drive integration features and challenges," Dig. The 26th Magnetic Recording Conf., p. A1, Minneapolis, MN, USA, 2015.

[161] A. A. Mamun, L. T. Heng, and T. G. Hung, "Efficient position encoding in hard disk drive using dual frequency servo bursts," 27th IEEE Ind. Electron. Soc. Conf., pp. 609-614, Denver, CO, USA, 2001.

[162] T. Hamaguchi, T. Ichihara, and H. Takano, "An accurate head-positioning signal for perpendicular recording using a dc-free servo pattern," J. Appl. Phys., vol. 91, no. 10, pp. 8697-8699, May 2002.

[163] E. C. Hughes and W. C. Messner, "Characterization of three servo patterns for position error signal generation in hard drives," Proc. American Control Conf., pp. 4317-4322, Denver, CO, USA, 2003.

[164] A. A. Mamun, T. H. Lee, G. X. Guo, W. E. Wong, and W. C. Ye, "Measurement of position offset in hard disk drive using dual frequency servo bursts," IEEE Trans. Instrum. Meas., vol. 52, no. 6, pp. 1870-1880, Dec 2003.

[165] W. E. Wong, L. Feng, Z. He, J. Liu, C. M. Kan, and G. Guo, "PC-based position error signal generation and servo system for a spinstand," IEEE Trans. Magn., vol. 41, no. 11, pp. 4315-4322, Nov 2005.

[166] S. Zhang and W. E. Wong, "Evaluation of servo pattern for perpendicular recording," in Asia-Pacific Magnetic Recording Conf., p. doi: 10.1109/APMRC.2006.365936, Singapore, 2006.

[167] A. Betts, "Null servo pattern," IBM Technical Disclosure Bulletin, vol. 18, no. 8, pp. 2656-2657, Jan 1976.

[168] M. Chen, Z. Wang, and D. T. Phan, "Disk drive servo control techniques to preserve PES continuity." U.S. Patent 7,616,399, Nov 10, 2009. 
[169] Y. Deng, M. X. Wang, and J. Zhang, "Triple track test for side erase band width and side erase amplitude loss of a recording head." U.S. Patent 7,907,361, Mar 15, 2011.

[170] C. Shi, J. Wong, and D. D. Terrill, "Method and system for measuring magnetic interference width." U.S. Patent 7,982,989, Jul 19, 2011.

[171] E. Cooper, "A nonlinearity specification for constructing the servo position error signal," IEEE Trans. Magn., vol. 33, no. 2, pp. 1104-1115, Mar 1997.

[172] A. W. Nazarian, T. P. Trieu, and R. K. Wong, "Self-PES linearity calibration method for MR head." U.S. Patent 5,946,158, Aug 31, 1999.

[173] S. Asgari and M. D. Hagen, "Disk drive with method of constructing a continuous position signal and constrained method of linearizing such position signal while maintaining continuity." U.S. Patent 6,369,974, Apr 9, 2002.

[174] T. J. Everett, "PES linearization scheme for disk drive servo using small excitation bode measurements." U.S. Patent 6,369,971, Apr 9, 2002.

[175] K. Ooi, W. Chiang, L. Xiu, S. Tan, and Y. Chen, "Method and apparatus for determining high order polynomials for linearization of position signals." U.S. Patent 6,674,607, Jan 6, 2004.

[176] H. T. Ho and T. Doan, "Distortion effects on servo position error transfer function," IEEE Trans. Magn., vol. 33, no. 4, pp. 2569-2572, Jul 1997.

[177] G. N. Boshnakov, "Some measures for asymmetry of distributions," Stat. Prob. Lett., vol. 77, no. 11, pp. 1111-1116, 2007.

[178] E. Cooper and R. Hampshire, "Adaptive compensation for position error signal nonlinearity," IEEE Trans. Magn., vol. 38, no. 3, pp. 1593-1602, May 2002.

[179] D. Cahalan and K. Chopra, "Effects of MR head track profile characteristics on servo performance," IEEE Trans. Magn., vol. 30, no. 6, pp. 4203-4205, Nov 1994.

[180] E. Lin and S. Li, "Characterizing head parameters of a disk drive by evaluating track profile of an overwritten track." U.S. Patent 8,717,695, May 6, 2014.

[181] J. M. Chue, D. Baral, and G. Dunbar, "Effect of erase bands on high tracks per inch applications," J. Appl. Phys., vol. 85, no. 8, pp. 5306-5308, Apr 1999. 
[182] M. R. Graham and R. A. de Callafon, "An iterative learning design for repeatable runout cancellation in disk drives," IEEE Trans. on Control Syst. Technol., vol. 14, no. 3, pp. 474-482, May 2006.

[183] C. Rea, P. Subedi, K. Gao, H. Zhou, P.-L. Lu, P. J. Czoschke, S. Hernandez, M. Ma, R. Lopusnik, Y. Peng, J.-U. Thiele, A. Q. Wu, G. Ju, T. Rausch, M. Seigler, and E. Gage, "Areal-density limits for heat-assisted magnetic recording and perpendicular magnetic recording," IEEE Trans. Magn., vol. 52, no. 7, pp. 1-4, Jul 2016. 


\section{Appendix A}

\section{Burst Distortion with 2 to $5 \mathrm{X}$ Harmonics}

In Chapter 5 section 3, we try to find out the effect on PES non-linearity of different kinds of burst shapes. We add $2 X$ to $5 X$ sin and $\cos$ harmonics as the distortion on top of the pure sinusoidal baseline burst to derive different burst shapes. For each harmonic, we set its amplitude to be $+/-10 \%$ of the baseline burst amplitude. The results for each distortion are listed in the following figures.
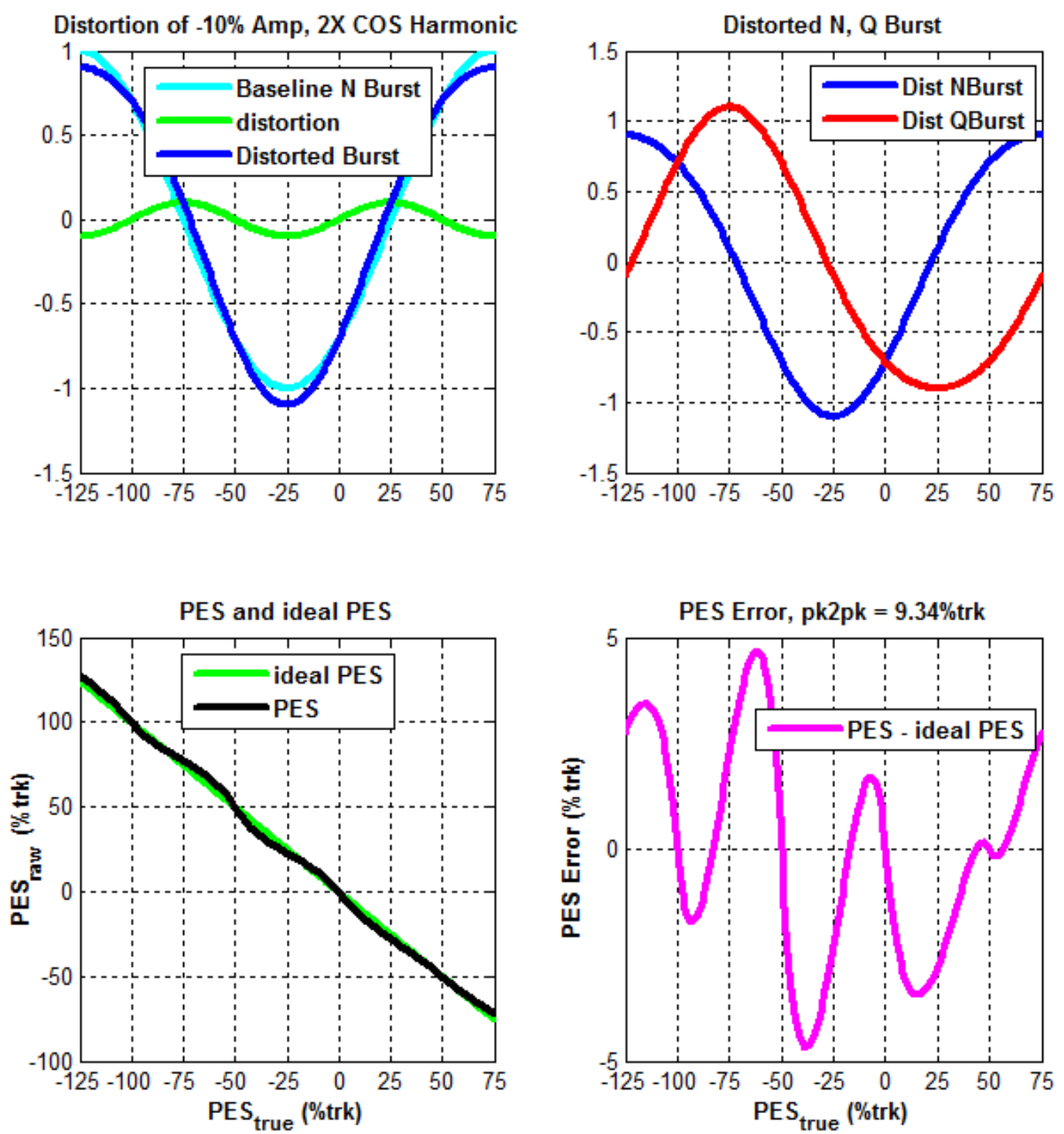

Figure A1: Demodulation and non-linearity of Null burst pattern with $-10 \%$ 2nd order cos harmonic distortion 

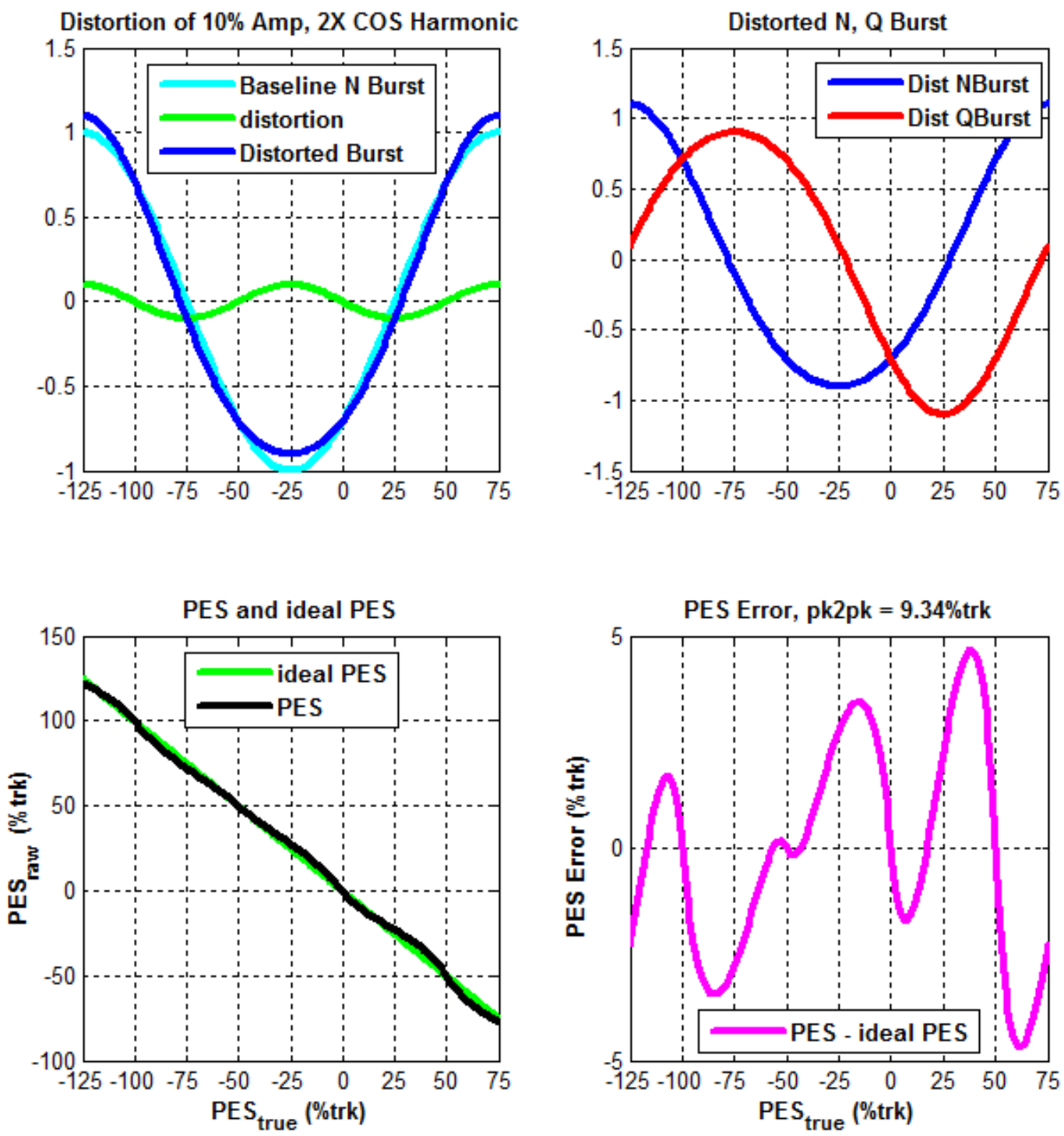

Figure A2: Demodulation and non-linearity of Null burst pattern with $10 \%$ 2nd order cos harmonic distortion 
Distortion of $-10 \%$ Amp, 2X SIN Harmonic

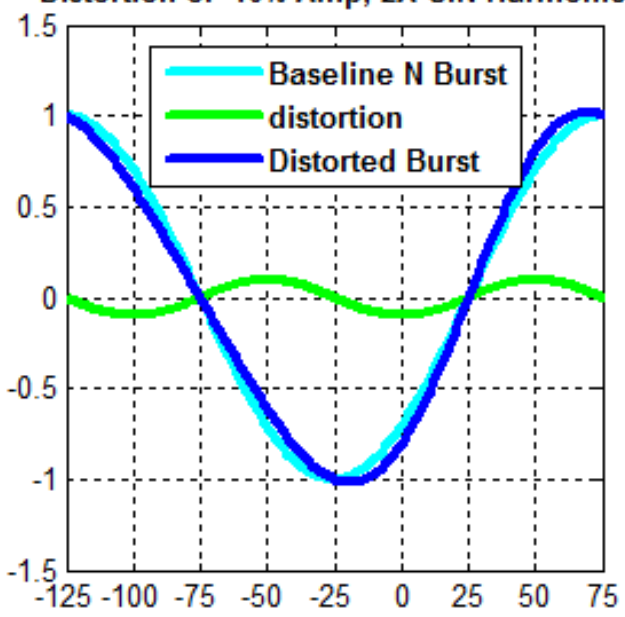

Distorted N, Q Burst

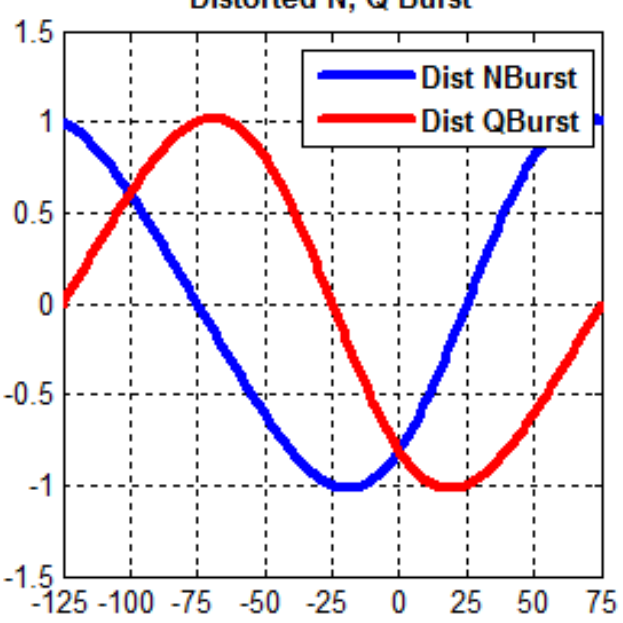

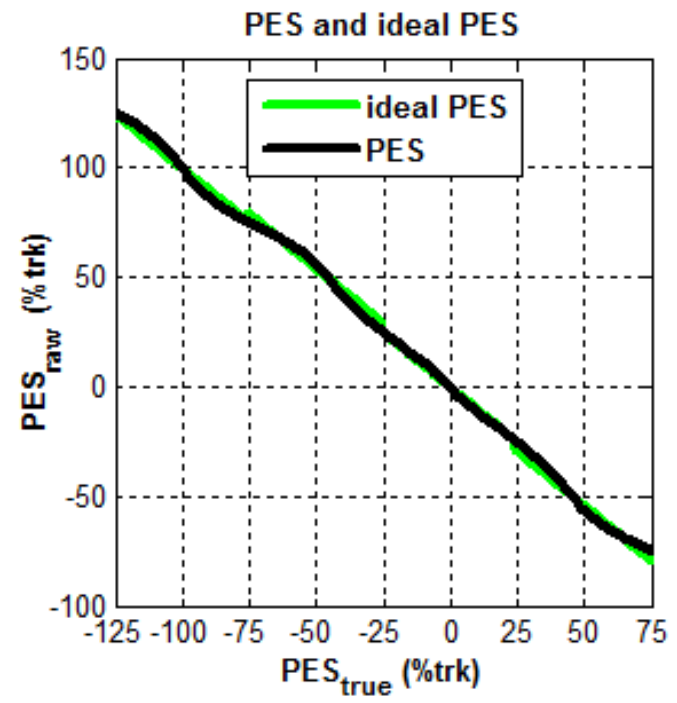

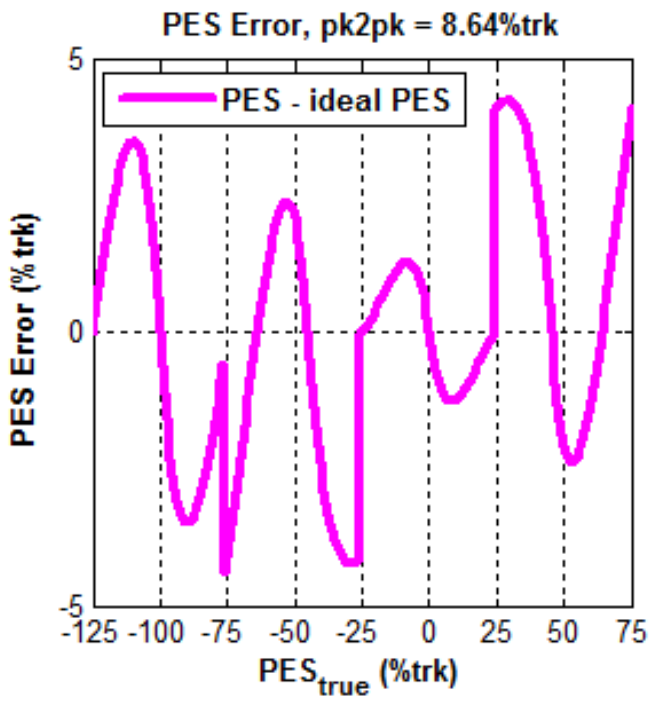

Figure A3: Demodulation and non-linearity of Null burst pattern with $-10 \%$ 2nd order sin harmonic distortion 

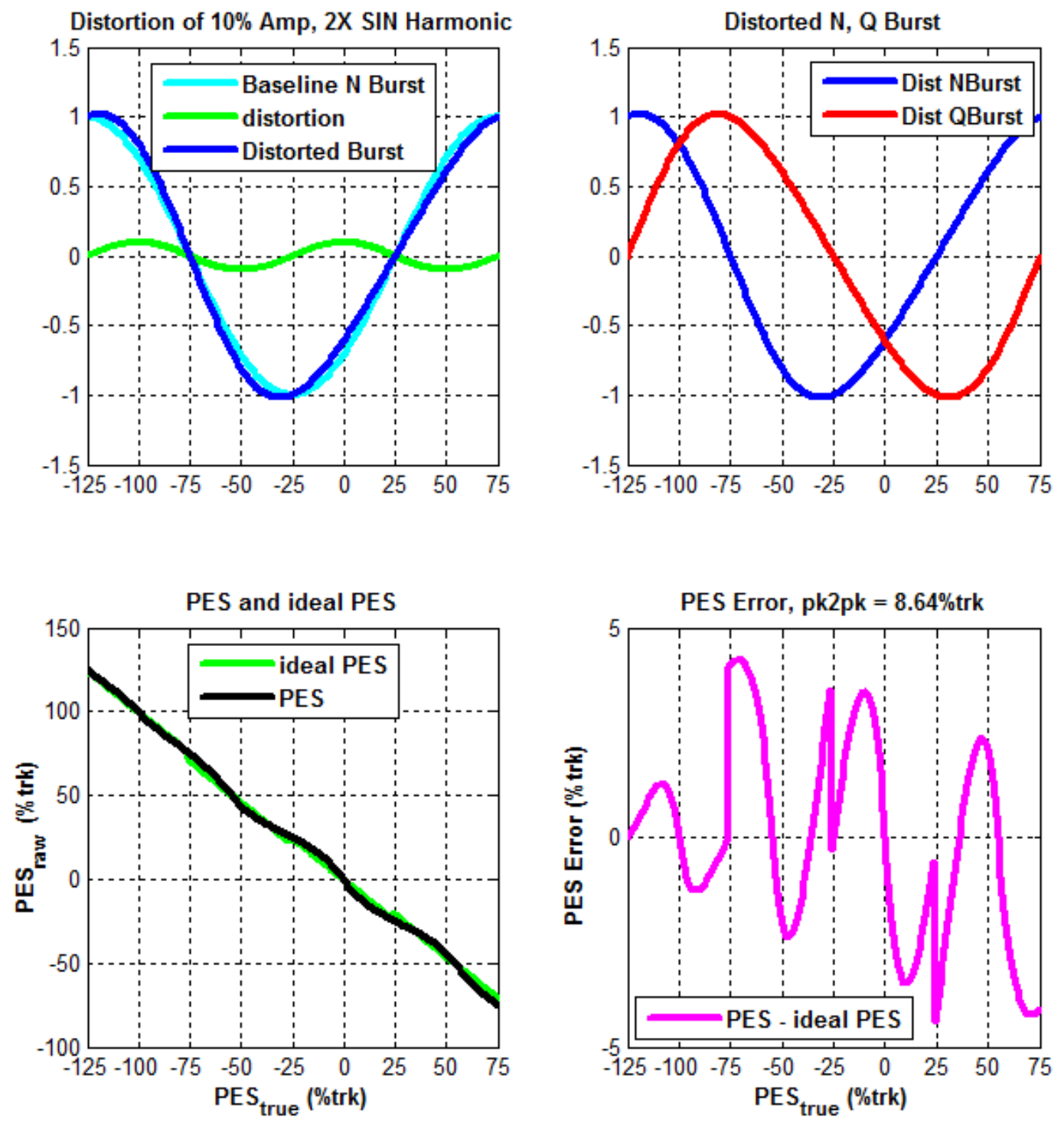

Figure A4: Demodulation and non-linearity of Null burst pattern with 10\% 2nd order sin harmonic distortion 

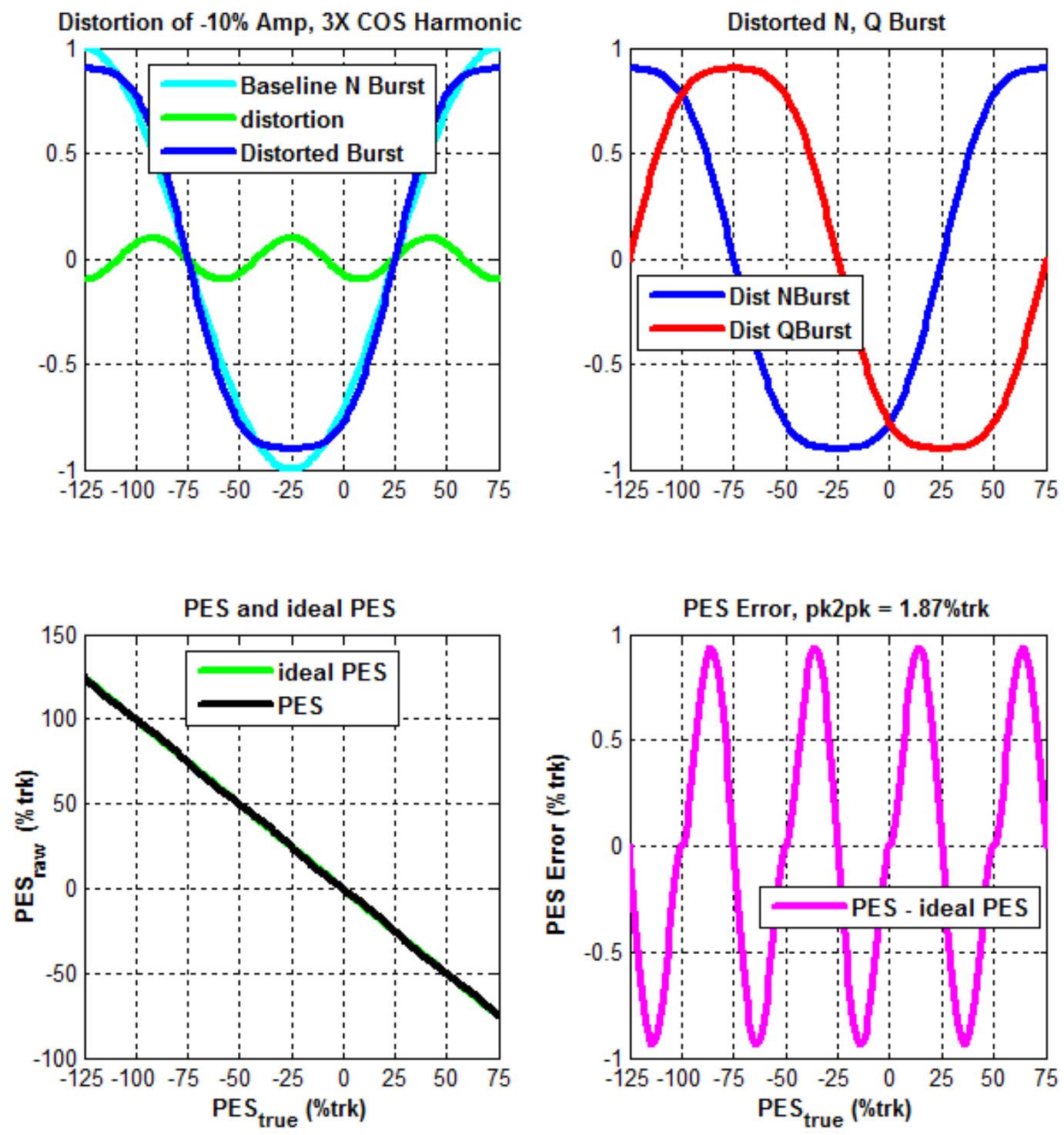

Figure A5: Demodulation and non-linearity of Null burst pattern with $-10 \%$ 3rd order cos harmonic distortion 
Distortion of $10 \%$ Amp, 3X COS Harmonic
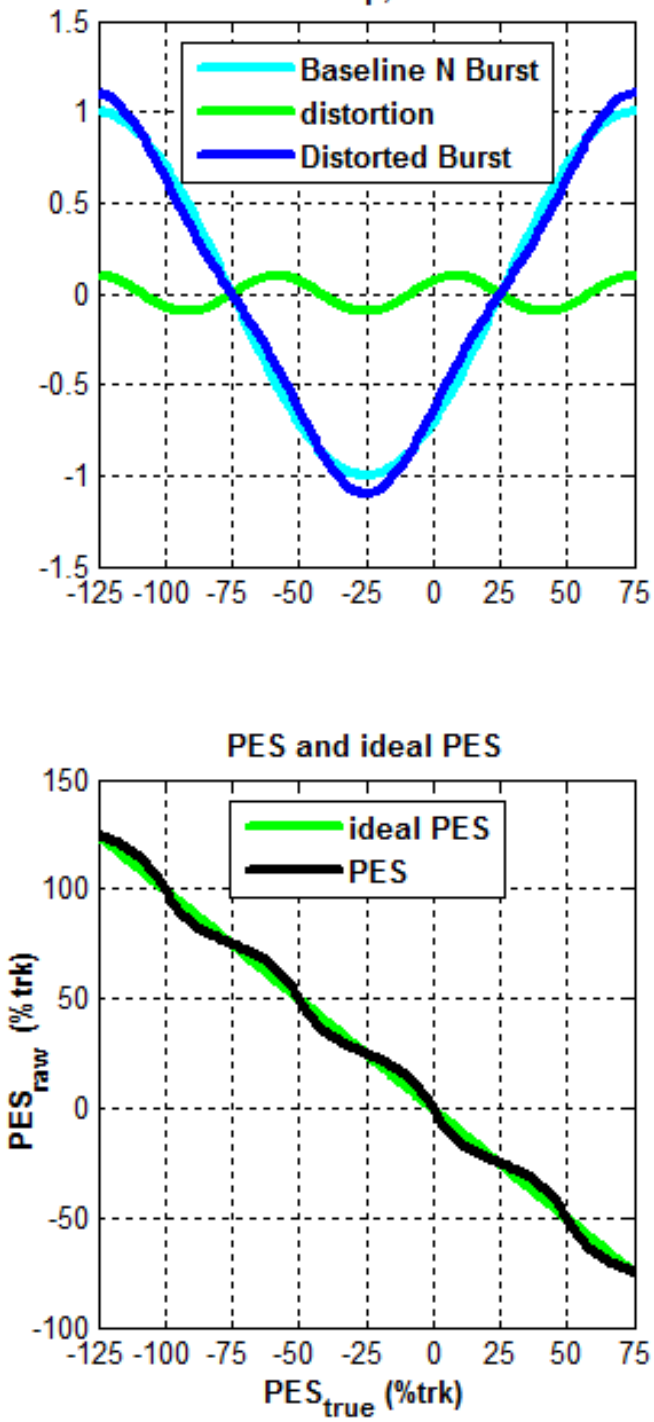

Distorted N, Q Burst
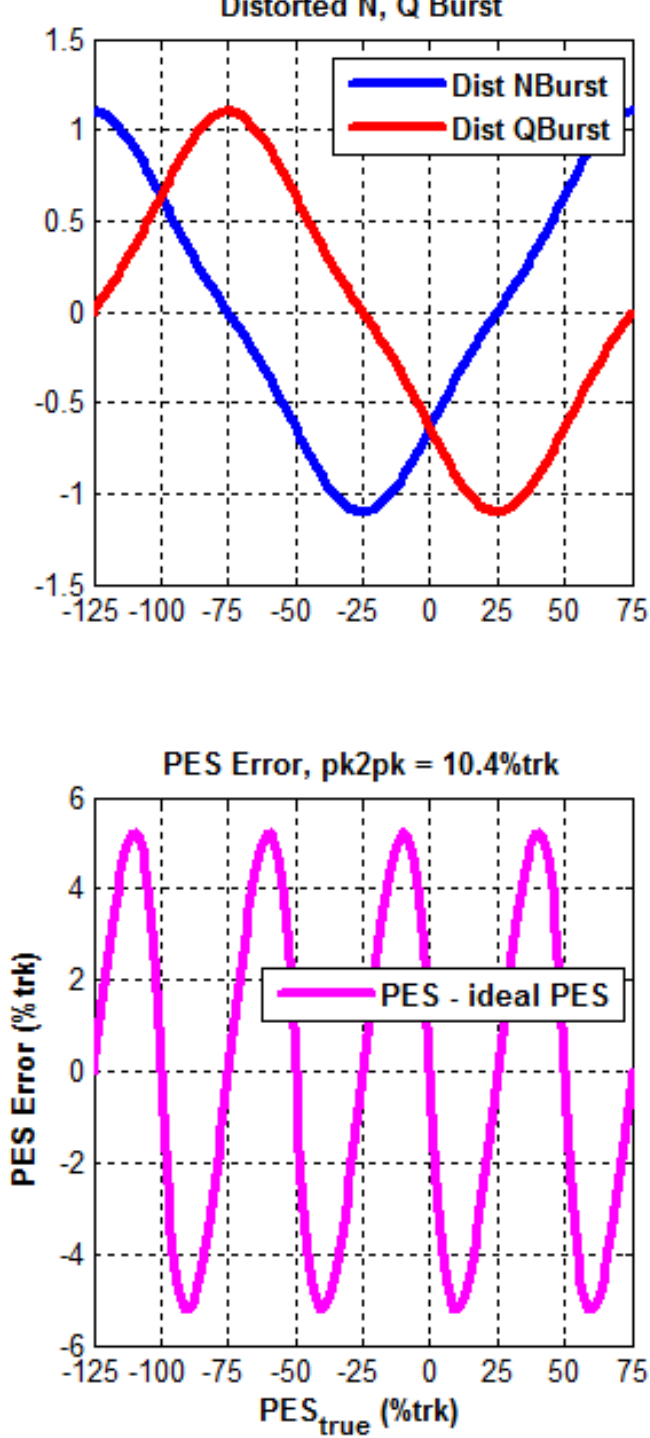

Figure A6: Demodulation and non-linearity of Null burst pattern with $10 \%$ 3rd order cos harmonic distortion 
Distortion of $-10 \%$ Amp, 3X SIN Harmonic
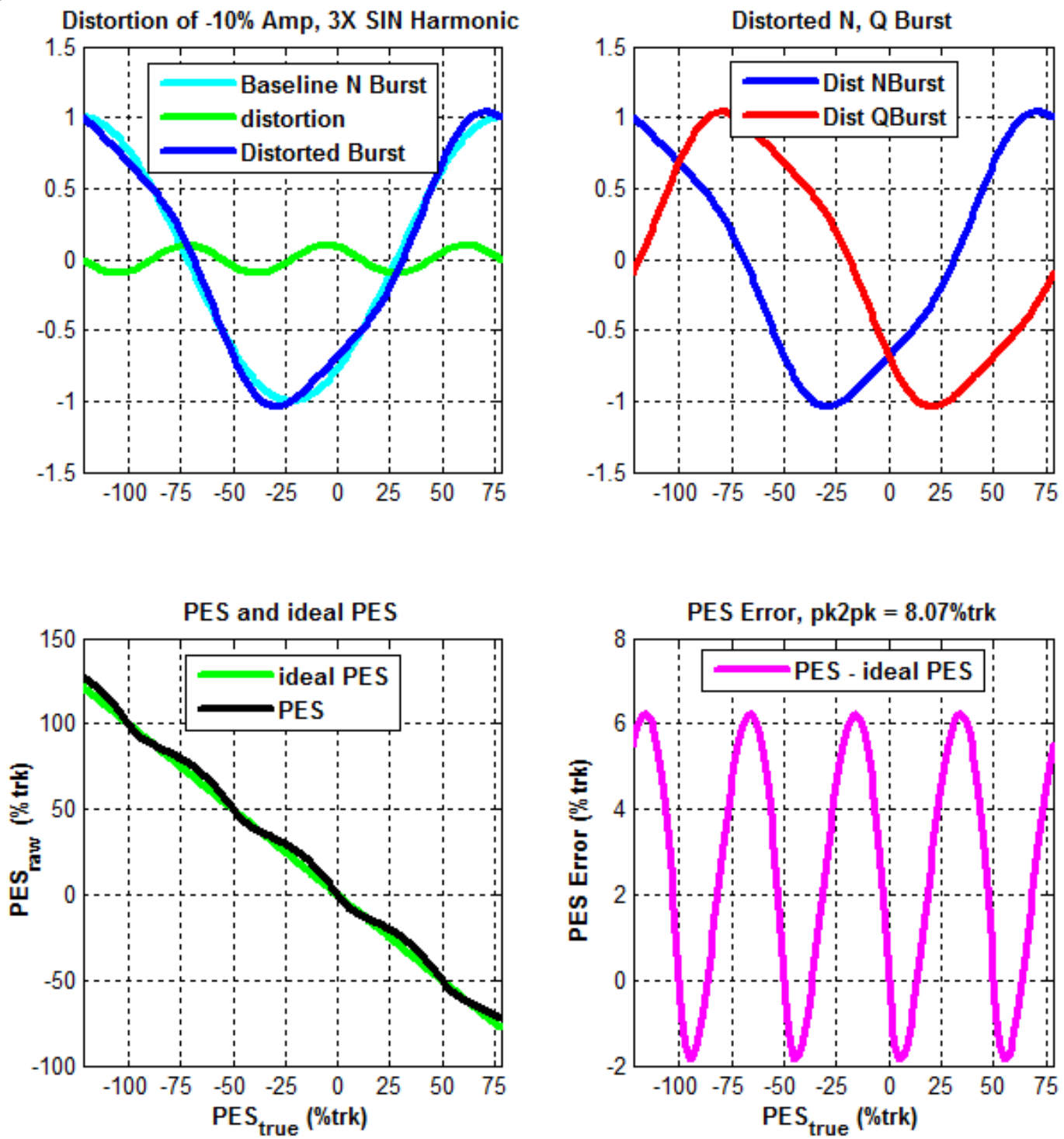

Figure A7: Demodulation and non-linearity of Null burst pattern with $-10 \%$ 3rd order sin harmonic distortion 

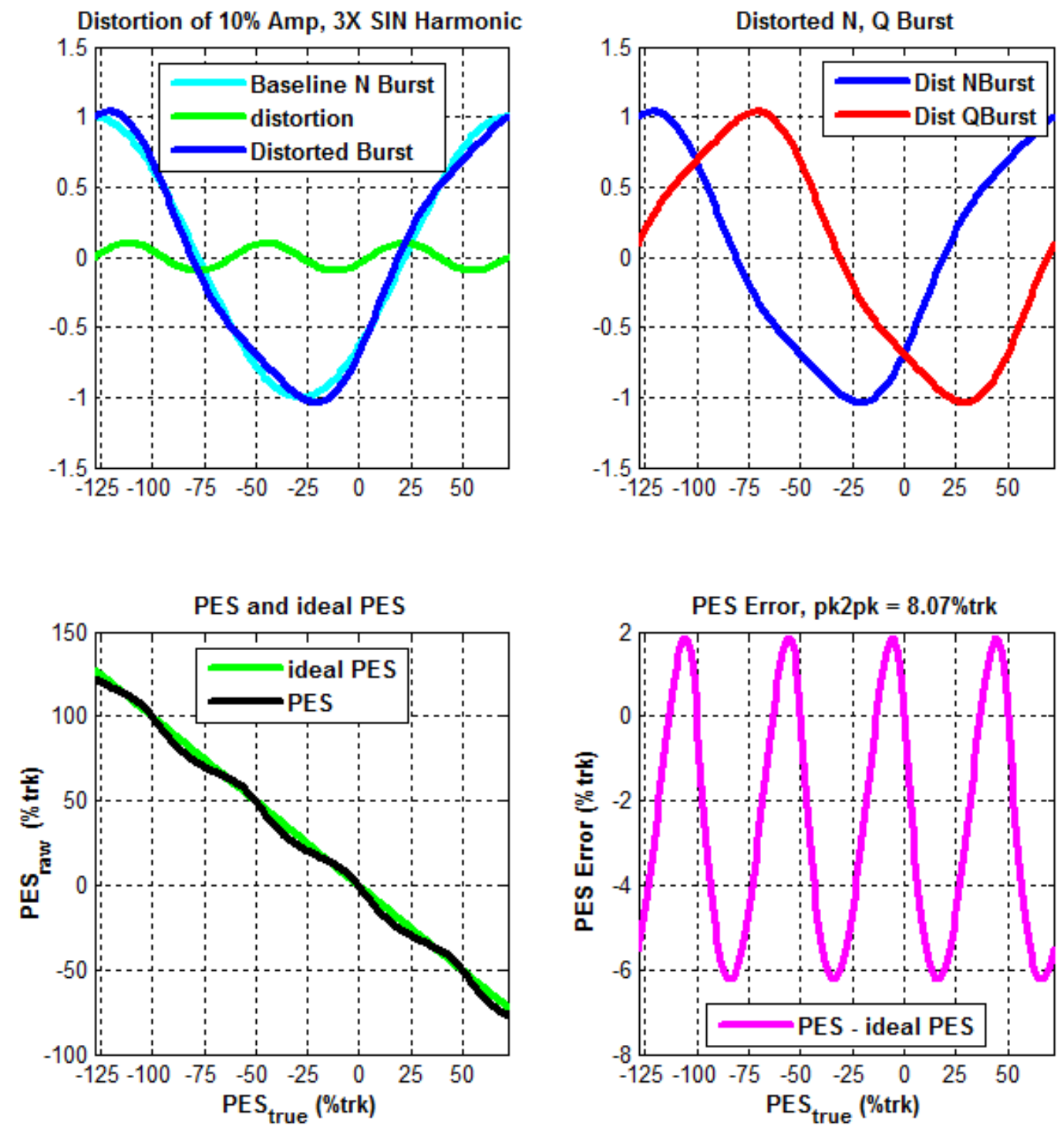

Figure A8: Demodulation and non-linearity of Null burst pattern with $10 \%$ 3rd order sin harmonic distortion 

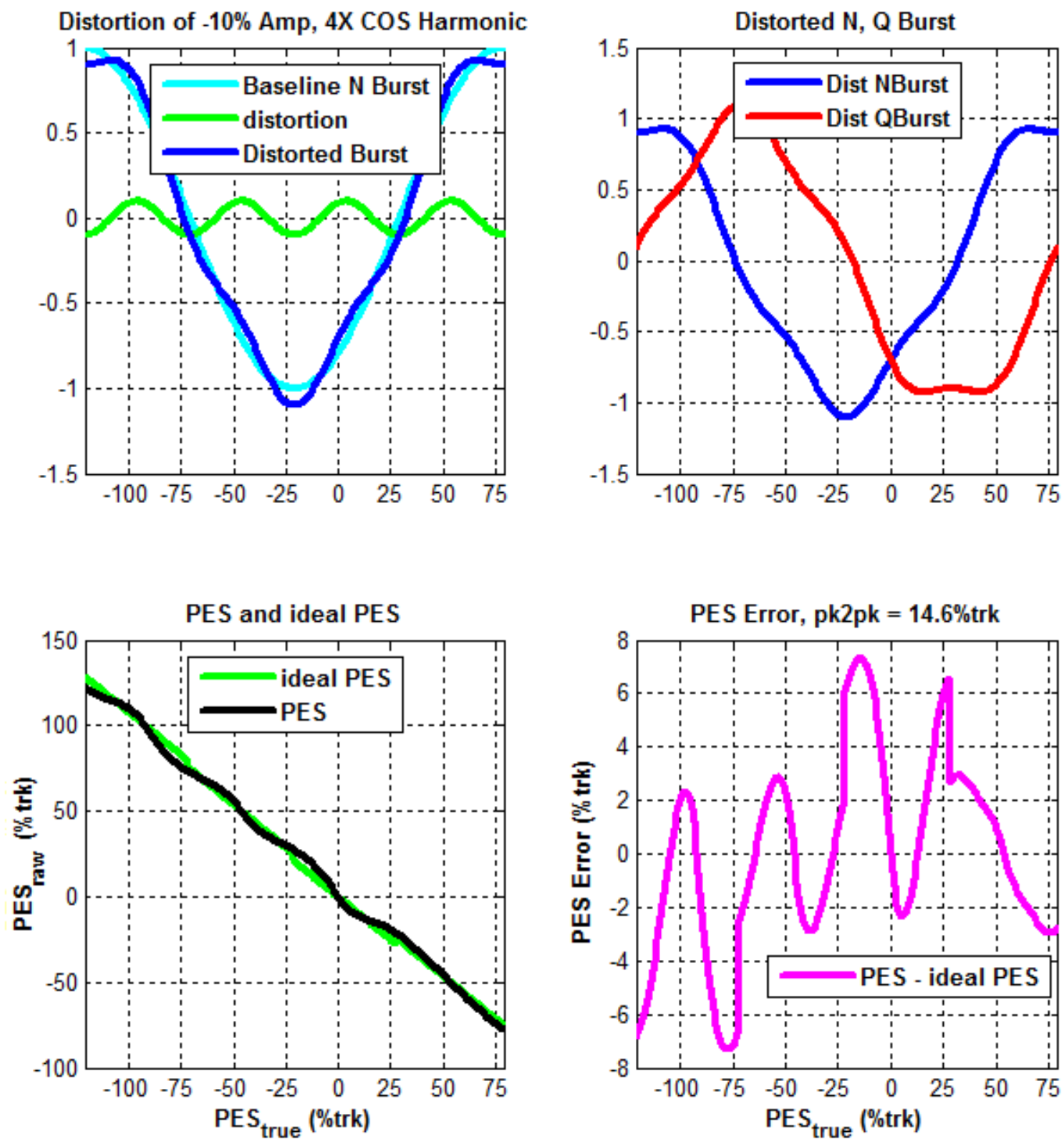

Figure A9: Demodulation and non-linearity of Null burst pattern with $-10 \%$ th order cos harmonic distortion 

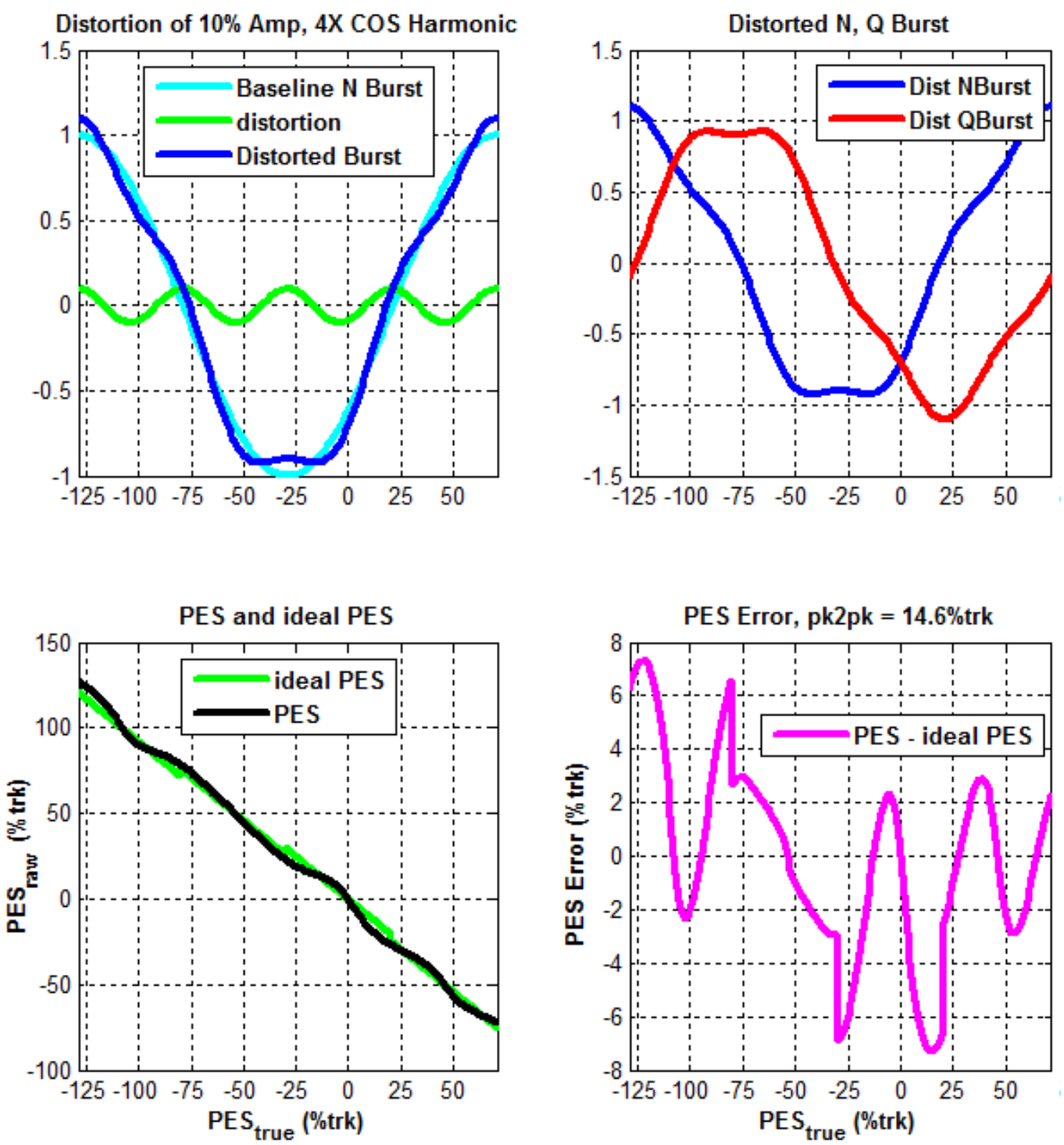

Figure A10: Demodulation and non-linearity of Null burst pattern with $10 \%$ th order cos harmonic distortion 
Distortion of -10\% Amp, 4X SIN Harmonic
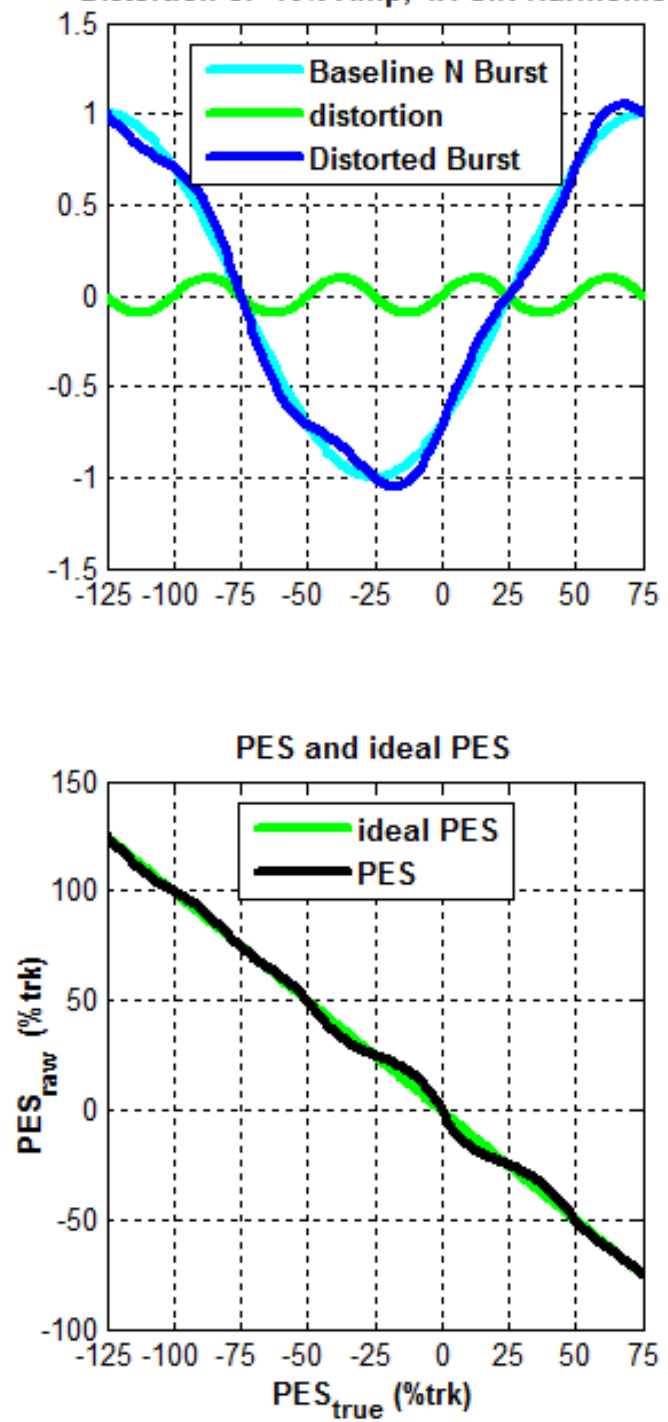
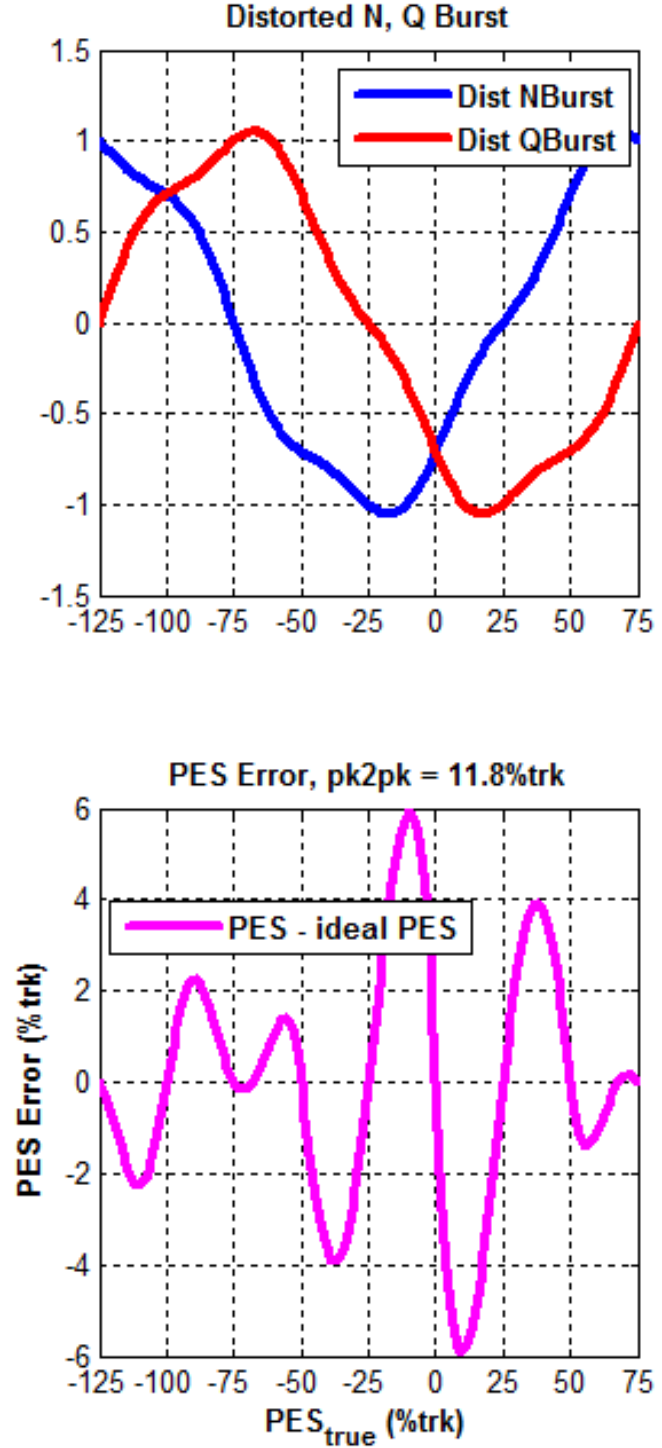

Figure A11: Demodulation and non-linearity of Null burst pattern with $-10 \%$ th order sin harmonic distortion 
Distortion of $10 \%$ Amp, 4X SIN Harmonic
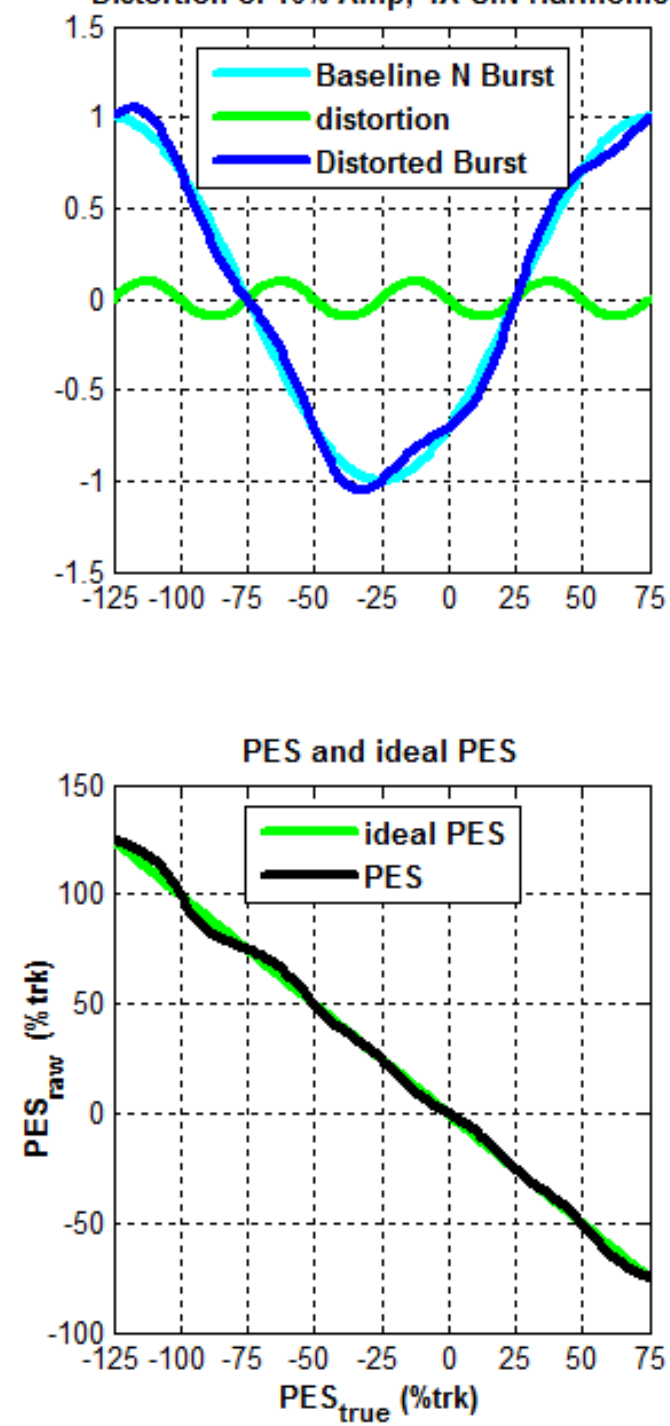
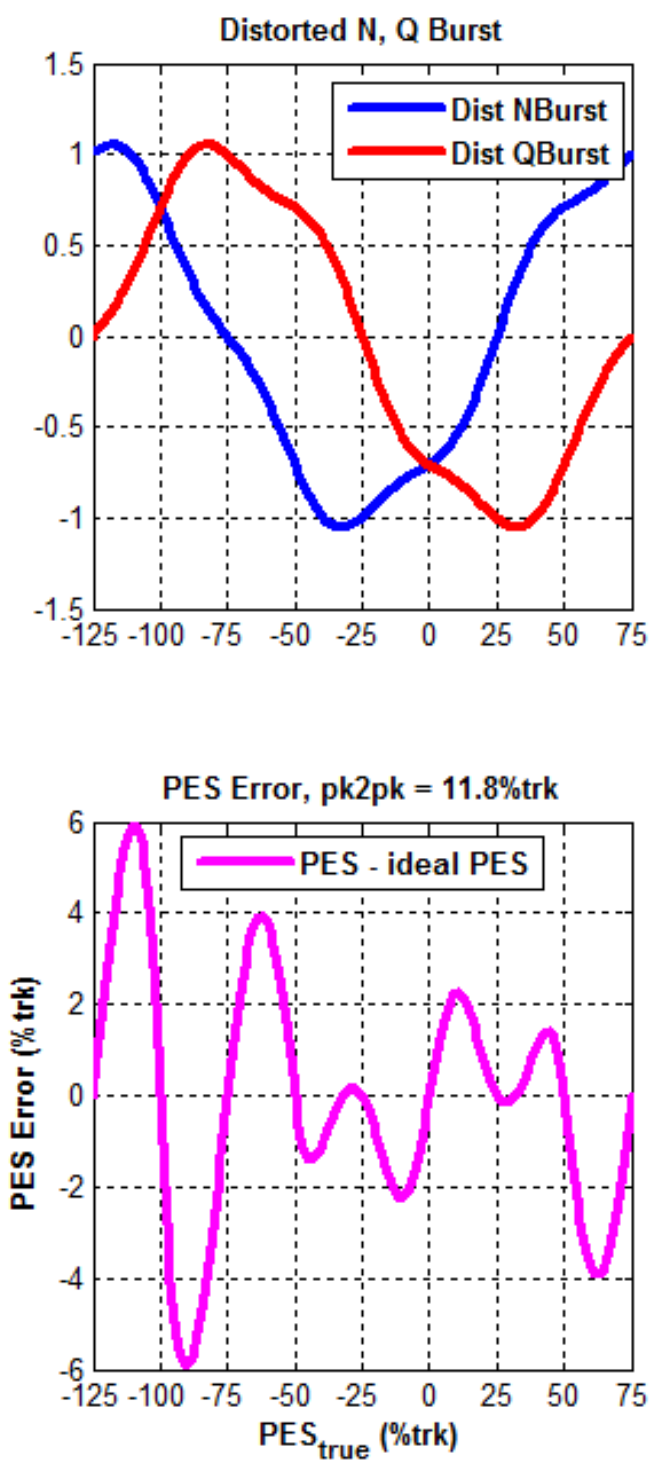

Figure A12: Demodulation and non-linearity of Null burst pattern with $10 \%$ 4th order sin harmonic distortion 
Distortion of $-10 \%$ Amp, $5 \mathrm{X}$ COS Harmonic
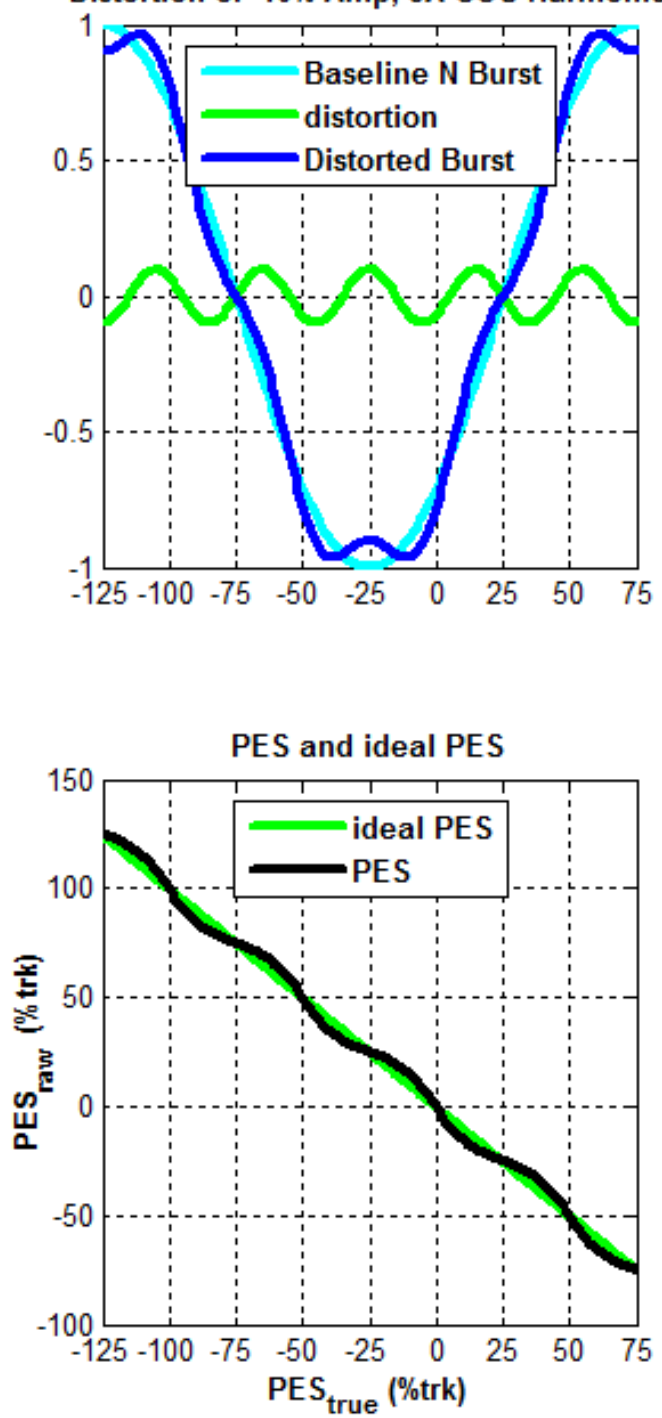
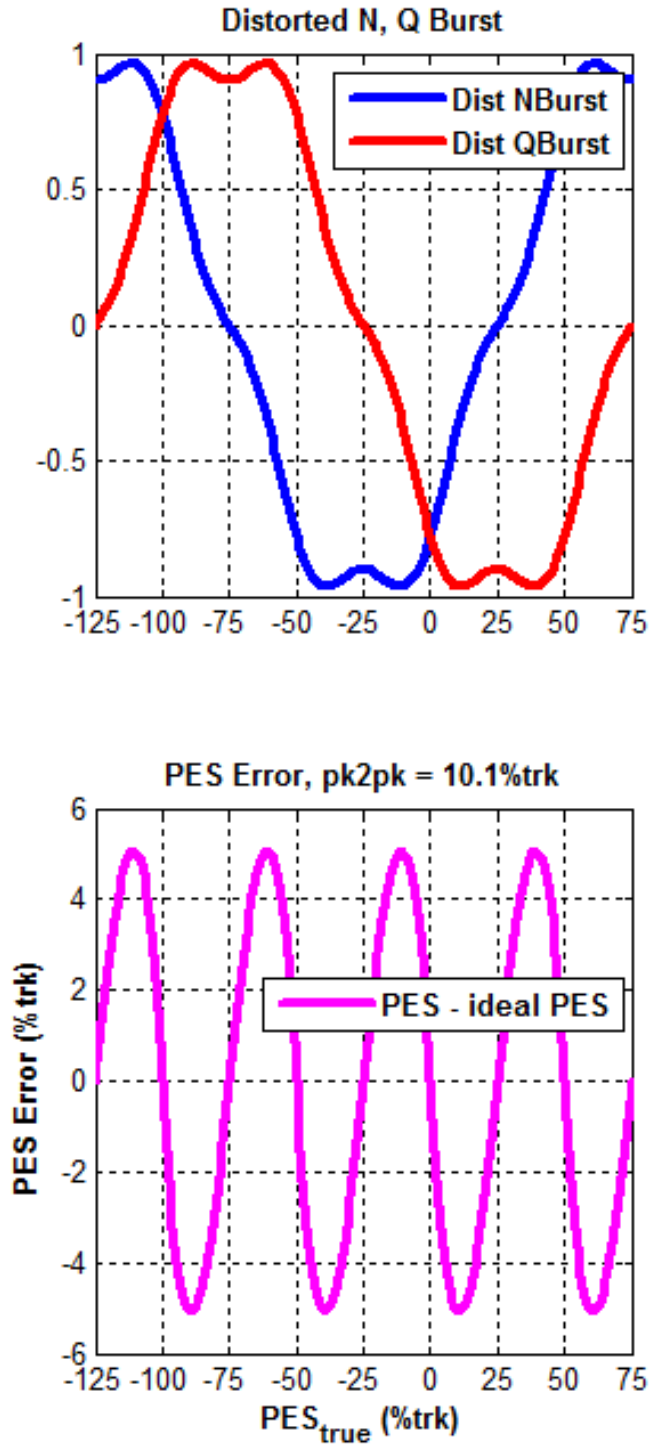

Figure A13: Demodulation and non-linearity of Null burst pattern with $-10 \%$ th order cos harmonic distortion 

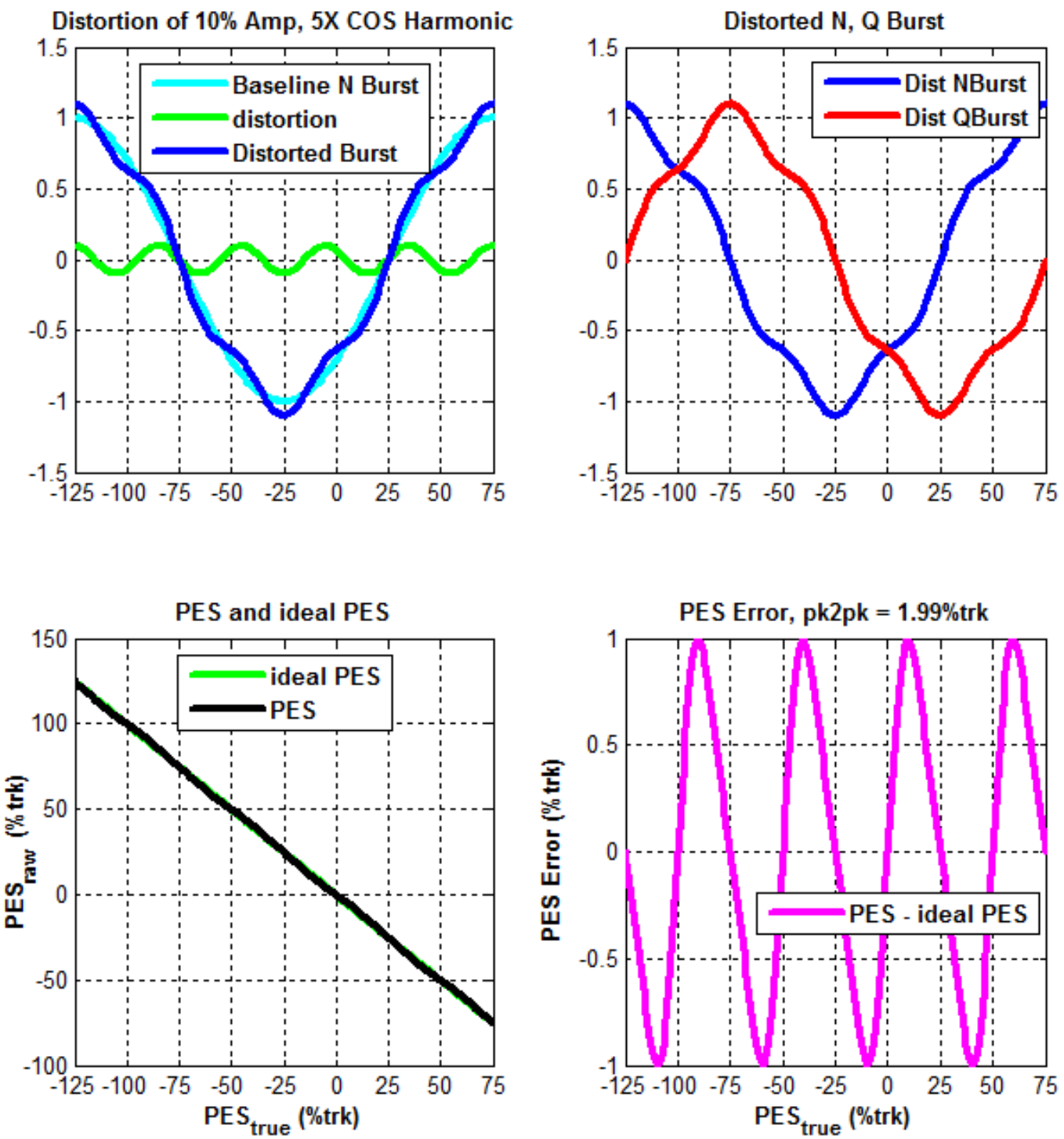

Figure A14: Demodulation and non-linearity of Null burst pattern with $10 \%$ th order cos harmonic distortion 
Distortion of $-10 \%$ Amp, 5X SIN Harmonic
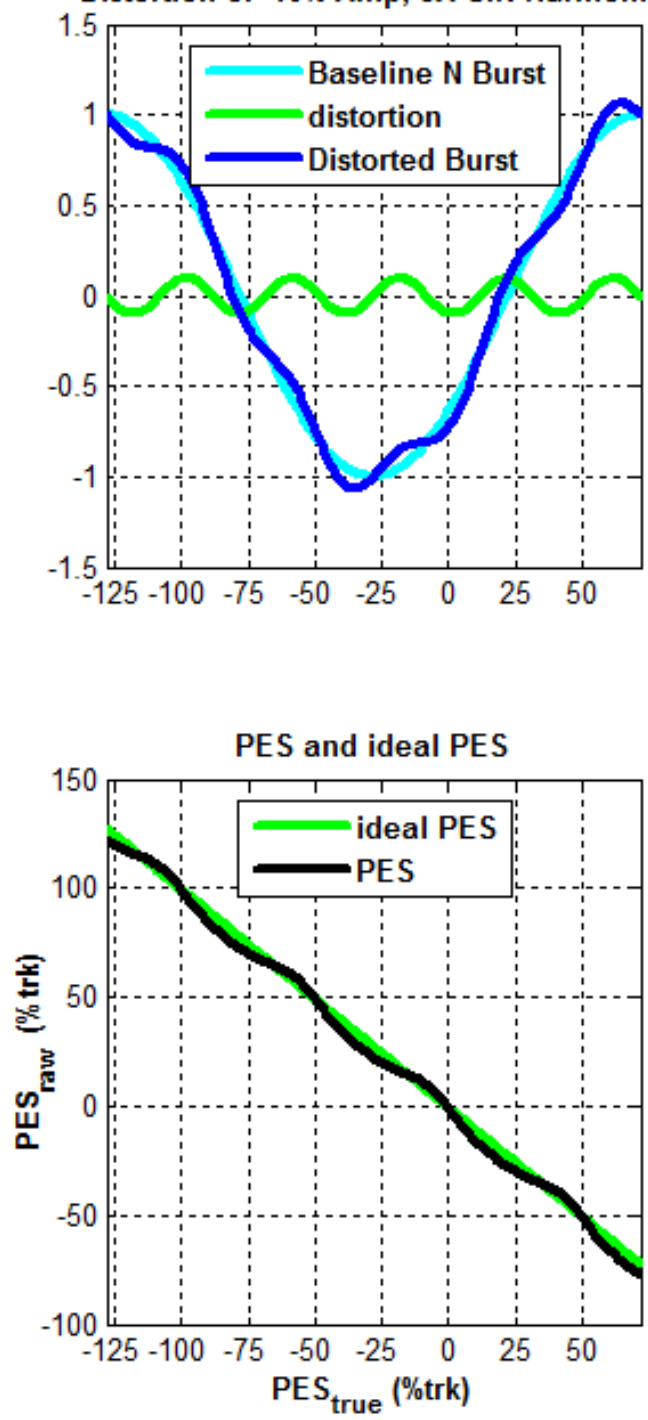
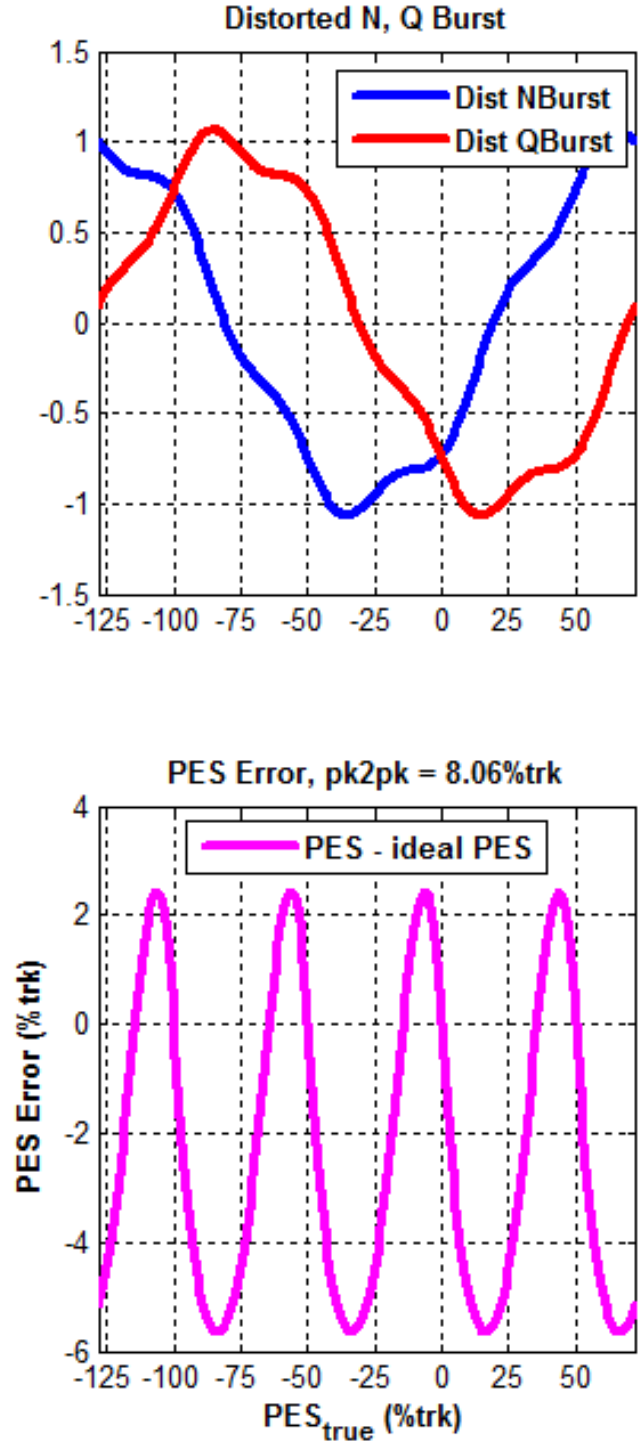

Figure A15: Demodulation and non-linearity of Null burst pattern with $-10 \% 5$ th order sin harmonic distortion 
Distortion of $10 \%$ Amp, 5X SIN Harmonic
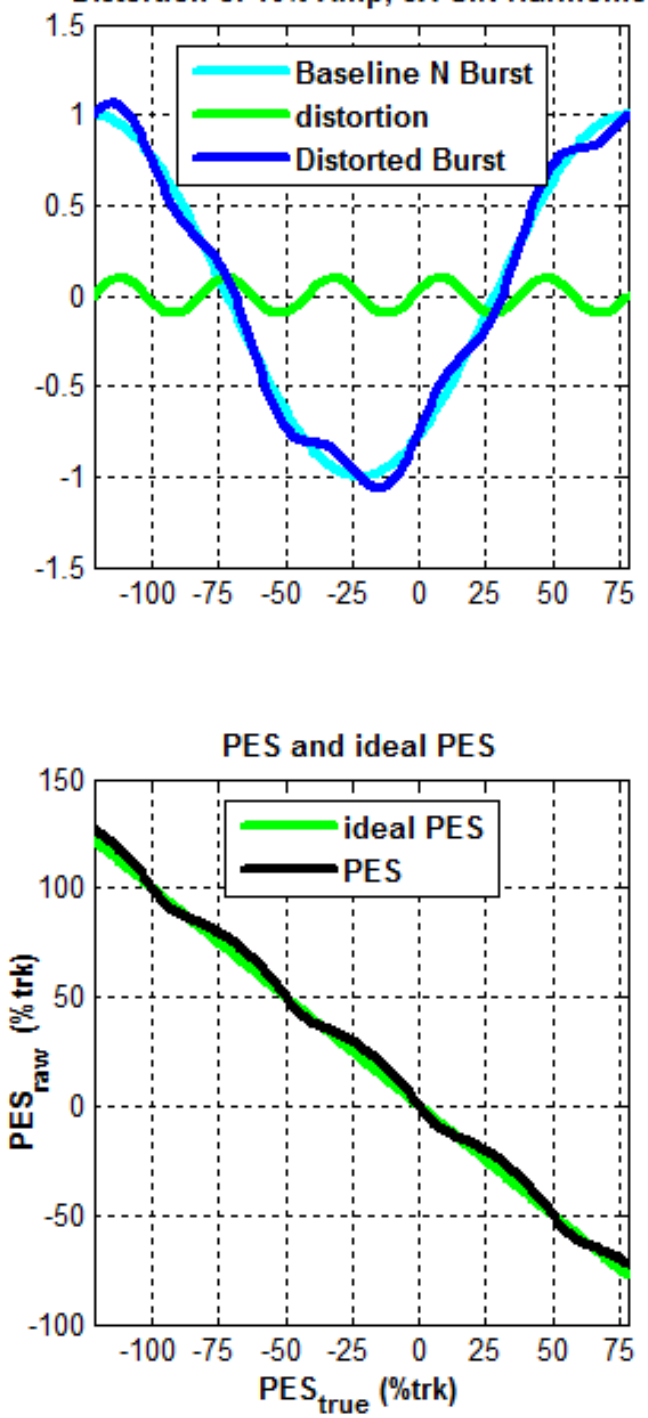
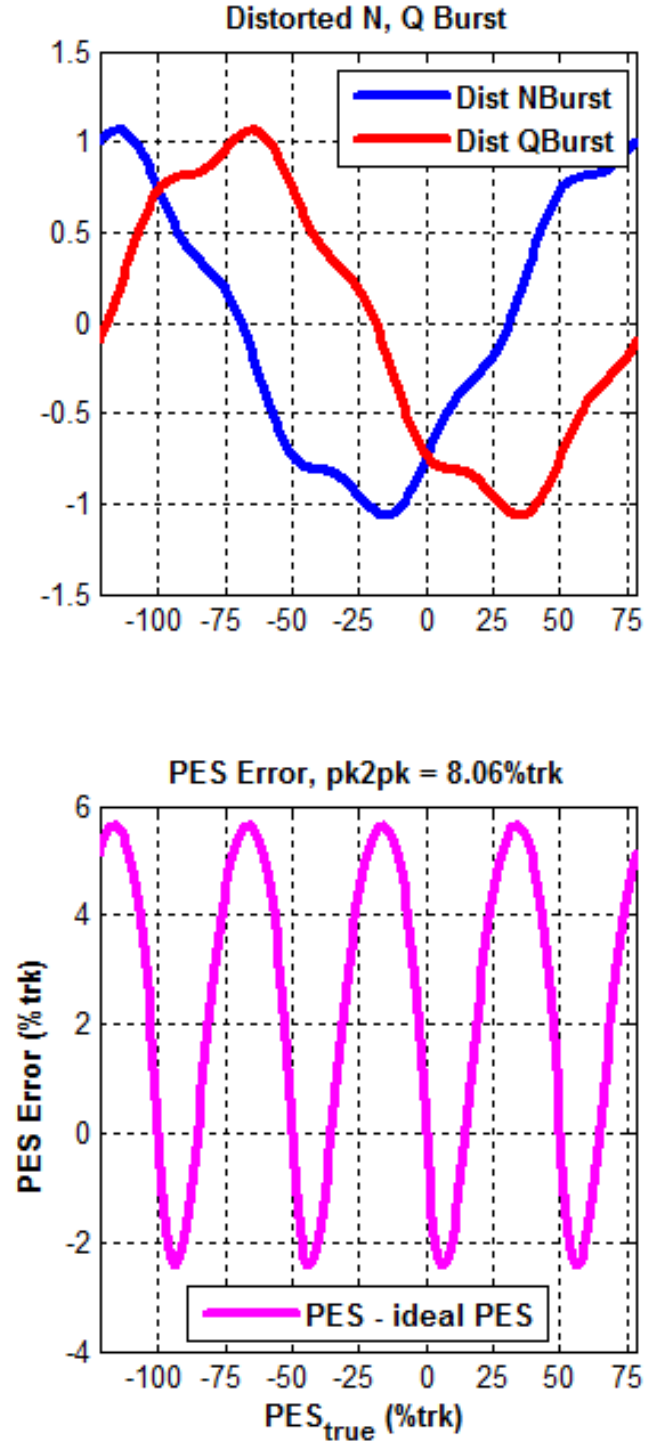

Figure A16: Demodulation and non-linearity of Null burst pattern with $10 \%$ th order sin harmonic distortion 
